\title{
Synthesis of Chiral Polycyclic Tetrahydrocarbazoles by Enantioselective Aminocatalytic Double Activation of 2-Hydroxycinnamaldehydes with Dienals
}

\author{
Yong-Chao Ming, ${ }^{\dagger}$ Xue-Jiao Lv, ${ }^{\dagger}$ Ming Liu, ${ }^{\dagger \ddagger}$ and Yan-Kai Liu ${ }^{\dagger, \ddagger}, *$ \\ ${ }^{\dagger}$ Molecular Synthesis Center \& Key Laboratory of Marine Drugs, Ministry of Education, School of \\ Medicine and Pharmacy, Ocean University of China, 5 Yushan Road, Qingdao 266003, China. \\ Laboratory for Marine Drugs and Bioproducts of Qingdao National Laboratory for Marine Science \\ and Technology, Qingdao 266003, China \\ Email: liuyankai@ouc.edu.cn (Y.-K. Liu)
}

\section{Supporting Information}




\section{Contents}

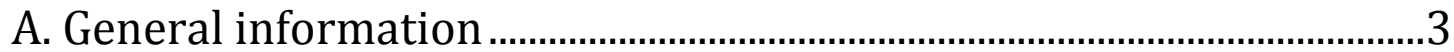

B. General procedure for the synthesis of enals 1 ........................................

C. Optimization of the reaction conditions ………..........................................

D. Scope of the reaction .................................................................................10

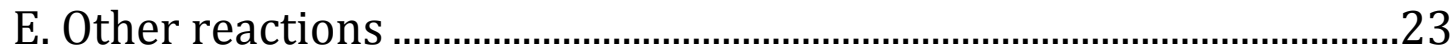

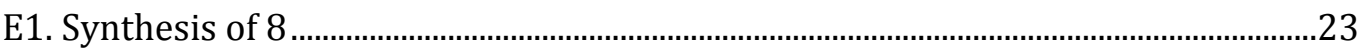

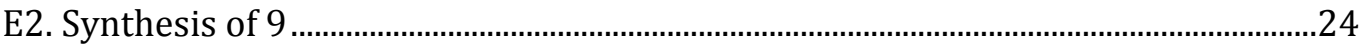

F. Synthetic transformation.......................................................................25

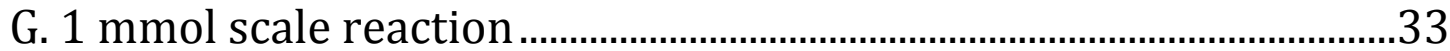

H. Control Experiments ................................................................................. 34

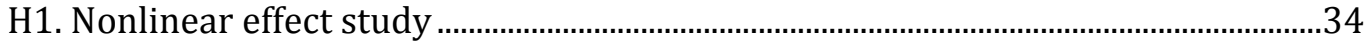

H2. Substrates by using -CN group instead of -CHO group ................................................34

I. Unreactive substituted 2,4-dienals............................................................35

J. NMR spectra and HPLC traces ..................................................................36

K. Single crystal X-Ray diffraction data .......................................................98

L. Proposed reaction mechanism ..................................................................101 


\section{A. General information}

The ${ }^{1} \mathrm{H}$ and ${ }^{13} \mathrm{C}$ NMR spectra were recorded at $500 \mathrm{MHz}$ or $400 \mathrm{MHz}$ for ${ }^{1} \mathrm{H}$ and at 125 $\mathrm{MHz}$ or $100 \mathrm{MHz}$ for ${ }^{13} \mathrm{C}$. The chemical shifts $(\delta)$ for ${ }^{1} \mathrm{H}$ and ${ }^{13} \mathrm{C}$ are given in ppm relative to residual signals of the solvents $\left(\mathrm{CDCl}_{3}\right.$ at $7.26 \mathrm{ppm}{ }^{1} \mathrm{H}$ NMR, $77.16 \mathrm{ppm}{ }^{13} \mathrm{C}$ NMR. $d_{6}$-DMSO at $2.50 \mathrm{ppm}{ }^{1} \mathrm{H}$ NMR, $\left.39.52 \mathrm{ppm}{ }^{13} \mathrm{C} \mathrm{NMR}\right)$. Coupling constants are given in $\mathrm{Hz}$. The following abbreviations are used to indicate the multiplicity: s, singlet; d, doublet; $t$, triplet; q, quartet; m, multiplet. High-resolution mass spectra (HRMS) were obtained from the Waters Q-Tof Ultima Global. X-ray data were obtained from Zhongke chemical technology service center. Optical rotations are reported as follows: $[\alpha]_{D}{ }^{20}$ (c in g per $100 \mathrm{~mL}$, solvent: $\left.\mathrm{CHCl}_{3}, \mathrm{MeOH}\right)$.

Note: NMR signals containing common solvent contaminants were list. $\mathrm{H}_{2} \mathrm{O}$ in $\mathrm{CDCl}_{3}$ at $1.56 \mathrm{ppm}{ }^{1} \mathrm{H}$ NMR, and in $d_{6}$-DMSO at $3.33 \mathrm{ppm}{ }^{1} \mathrm{H}$ NMR; Ethyl acetate in $\mathrm{CDCl}_{3}$ at 2.05 (s), 4.12 (q), 1.26 (t) ppm ${ }^{1} \mathrm{H}$ NMR; $\mathrm{CH}_{2} \mathrm{Cl}_{2}$ in $\mathrm{CDCl}_{3}$ at 5.30 (s) ppm ${ }^{1} \mathrm{H}$ NMR; Grease in $\mathrm{CDCl}_{3}$ at $0.86(\mathrm{~m}), 1.26(\mathrm{br}, \mathrm{s}) \mathrm{ppm} 1 \mathrm{H} \mathrm{NMR}$.

All the reactions were set up under air and using commercial solvents, without any precautions to exclude moisture, unless otherwise noted open air chemistry on the bench-top. Chromatographic purification of products was accomplished using force-flow chromatography (FC) on silica gel (300-400 mesh). For thin layer chromatography (TLC) analysis throughout this work, Merck pre-coated TLC plates (silica gel 60 GF254, 0.25 $\mathrm{mm}$ ) were used, using UV light as the visualizing agent and a phosphomolybdic acid or basic aqueous potassium permanganate $\left(\mathrm{KMnO}_{4}\right)$ as stain developing solutions. Organic solutions were concentrated under reduced pressure on a Büchi rotary evaporator.

HPLC analyses on chiral stationary phase were performed on a Hitachi Chromaster. Daicel Chiralpak IA, IB, IC, ID, OD-H or AD columns with $n$-hexane/i-PrOH as the eluent were used. HPLC traces were compared to racemic samples which prepared by mixture of two enantiomeric final products obtained using $(S)$ and $(R)$ catalyst.

Commercial reagents and solvents were purchased from Sigma Aldrich, Fluka, and Alfa Aesar used as received, without further purification. The 2-hydroxyinnamaldehyde 2 were prepared according to literature procedures. ${ }^{[1]}$ 


\section{B. General procedure for the synthesis of enals 1}

\section{The synthesis of enals 1a-1r:}

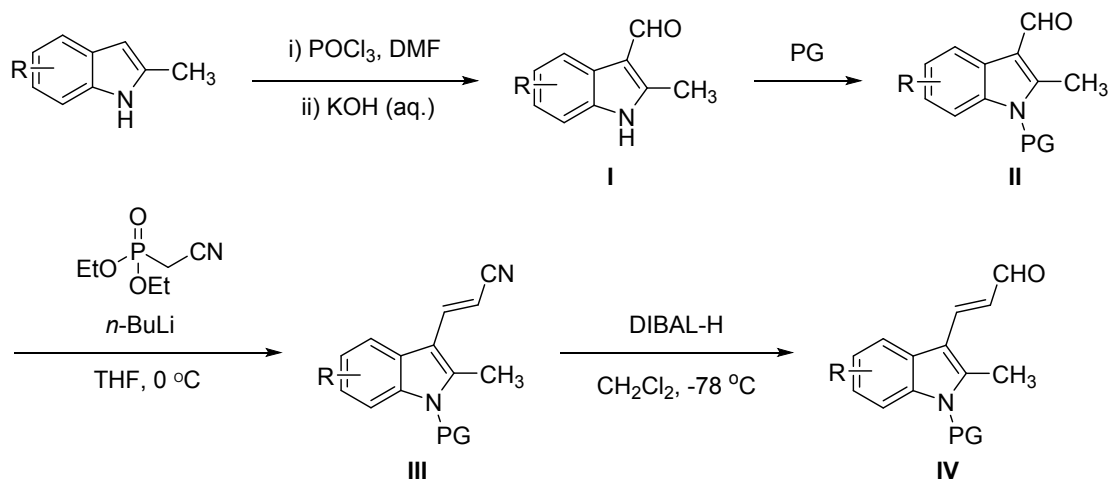

Step 1: Phosphorus oxychloride (12.5 mmol, 2.5 equiv.) was added dropwise to dimethyl formamide ( $5 \mathrm{~mL}$ per $1 \mathrm{~mL}$ of $\mathrm{POCl}_{3}$ ) with ice-bath cooling under anhydrous conditions. The mixture was stirred for 5 minutes then the chosen indole $(5.0 \mathrm{mmol}, 1.0$ equiv.) was added as a dimethyl formamide solution (10 $\mathrm{mL}$ per $1 \mathrm{~g}$ of indole). The mixture was then allowed to warm to room temperature and stirred for 3 hours. $3.8 \mathrm{M}$ aqueous potassium hydroxide (10.0 equiv.) was added via a dropping funnel and the mixture was heated to reflux in oil bath overnight. It was cooled to room temperature before adding saturated solution of $\mathrm{NaHCO}_{3}$. The aqueous layer was extracted with ethyl acetate and the combined organic layers were dried over sodium sulfate and filtered. Crude product I was obtained by vacuum distillation and put into the next step.

Step 2: (1) PG = Me: To a tetrahydrofuran solution of I (5.0 mmol, 1.0 equiv.), $\mathrm{NaH}$ (10.0 mmol, 2.0 equiv.) was added in portion at $0{ }^{\circ} \mathrm{C}$ in ice bath, and the mixture was stirred for further $0.5 \mathrm{~h}$. Then MeI (10 mmol, 2.0 equiv.) was added to the mixture at $0{ }^{\circ} \mathrm{C}$. The reaction mixture was stirred for 6 hours at room temperature and quenched by $\mathrm{H}_{2} \mathrm{O}$ ( 3 $\mathrm{mL}$ ). The solvent was removed after reduced pressure and the residue was extracted with ethyl acetate. The organic layer was concentrated in vacuum to furnish the desired product II that required no further purification. (2) PG = Boc: To an acetonitrile solution of I $\quad(5.0$ mmol, 1.0 equiv. $), \quad(B o c)_{2} \mathrm{O} \quad(10.0 \quad \mathrm{mmol}, 2.0$ equiv. $)$ and 4-dimethylaminopyridine were added systematically at $0{ }^{\circ} \mathrm{C}$ in ice bath. After the addition, the reaction mixture was stirred at room temperature for 3 hours. The solvent 
was extracted with ethyl acetate and $\mathrm{H}_{2} \mathrm{O}$. The organic layer was concentrated in vacuum to furnish the desired product II

Step 3: To a solution of diethylcyanomethyl phosphonate (6.5 mmol, 1.3 equiv.) in anhydrous THF $(10 \mathrm{~mL})$ at $0{ }^{\circ} \mathrm{C}, n$-BuLi $(6.0 \mathrm{mmol}, 1.2$ equiv., $2.5 \mathrm{M}$ in hexanes) was added dropwise and the mixture was stirred for 1 hour at the same temperature. A solution of the aldehyde (1.0 equiv.) in anhydrous THF $(10 \mathrm{~mL})$ was prepared and the solution of the Wittig reagent II (5mmol, 1.0 equiv.) was added dropwise via cannula. After the addition, the reaction mixture was stirred at $0{ }^{\circ} \mathrm{C}$ for 4 hours. The reaction was quenched with $\mathrm{NH}_{4} \mathrm{Cl}$, then extracted with ethyl acetate and water and dried over anhydrous $\mathrm{Na}_{2} \mathrm{SO}_{4}$. Crude product III was obtained by vacuum distillation and put into the next step.

Step 4: A solution of the nitrile III (5.0 mmol, 1.0 equiv.) in anhydrous toluene (20 mL) was cooled down to $-78^{\circ} \mathrm{C}$ and at that temperature, a solution of diisobutylaluminium hydride DIBAL in heptane ( $6 \mathrm{mmol}, 1.2$ equiv.) was added dropwise. The reaction mixture was stirred at $-78{ }^{\circ} \mathrm{C}$ for 4 hours. Then, the reaction was quenched with methanol (2 mL) and $\mathrm{H}_{2} \mathrm{O}(2 \mathrm{~mL})$. The solution was filtered, evaporated and purified by column chromatograph on a silica gel to give the desired product IV.

The synthesis of enals $1 \mathrm{~s}$ and $1 \mathrm{t}$ :

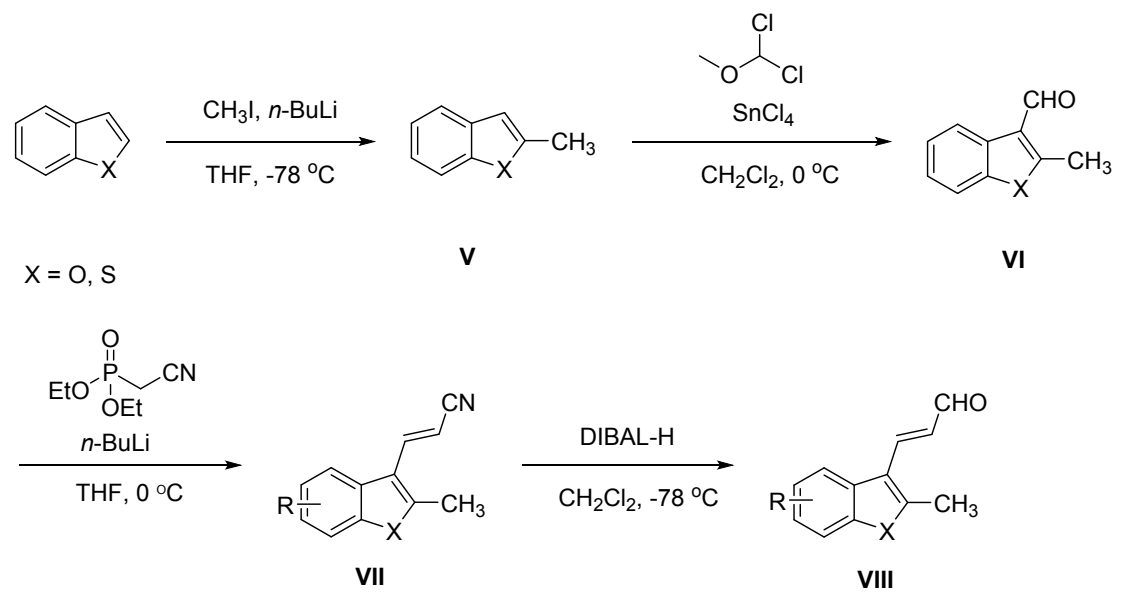

Step 1: Under the condition of full nitrogen, $n$-BuLi (6.0 mmol, 1.2 equiv.) was dropped into the THF solution of raw material ( $5.0 \mathrm{mmol}, 1.0$ equiv.) and reacted at $-78{ }^{\circ} \mathrm{C}$ for 1 hour. Then methyl iodide ( $6.0 \mathrm{mmol}, 1.2$ equiv.) was added to the reaction solution for 1 hour at room temperature. The reaction was quenched with $\mathrm{NH}_{4} \mathrm{Cl}$, then extracted with 
ethyl acetate and water and dried over anhydrous $\mathrm{Na}_{2} \mathrm{SO}_{4}$. Crude product $\mathbf{V}$ was obtained by vacuum distillation and put into the next step.

Step 2: Under the condition of full nitrogen, 1,1-dichlorodimethyl ether (7.5 mmol, 1.5 equiv.) was added to $\mathrm{CH}_{2} \mathrm{Cl}_{2}$ solution of $\mathbf{V}$ (5.0 mmol, 1.0 equiv.), and then $\mathrm{SnCl}_{4}$ (7.5 mmol, 1.5 equiv.) was added. The reaction was carried out for 2 hours at room temperature (monitored by TLC). Then the reaction was quenched with saturated $\mathrm{NaHCO}_{3}$ solution at $0{ }^{\circ} \mathrm{C}$ in ice bath, filtered with diatomite, extracted with $\mathrm{CH}_{2} \mathrm{Cl}_{2}$ and dried over anhydrous $\mathrm{Na}_{2} \mathrm{SO}_{4}$. The crude product VI was purified by column chromatography.

Step 3-4: Procedures for the synthesis of VII-VIII were performed as the synthesis of III and IV

[1] Chen, Y.-H.; Sun, X.-L.; Guan, H.-S.; Liu, Y.-K., J. Org. Chem. 2017, 82, 4774. 


\section{Optimization of the reaction conditions}

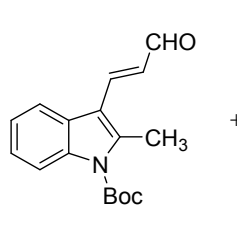

$1 \mathrm{a}$

$0.12 \mathrm{mmol}$<smiles>O=C/C=C/c1ccccc1O</smiles>

2a

$0.1 \mathrm{mmol}$
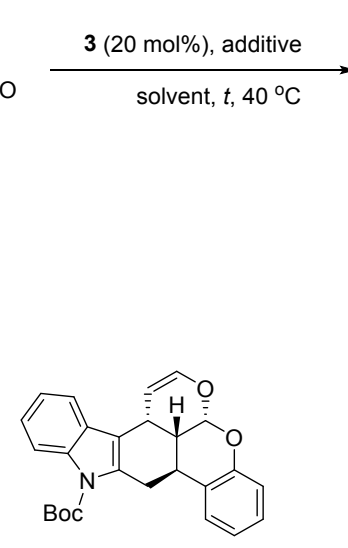

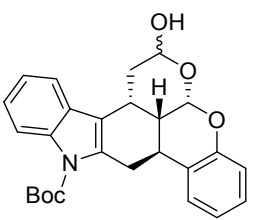

$5 a$

3a $\mathrm{Ar}=\mathrm{Ph}, \mathrm{R}=\mathrm{TMS}$

3b $\mathrm{Ar}=3,5-\left(\mathrm{CF}_{3}\right)_{2} \mathrm{C}_{6} \mathrm{H}_{3}, \mathrm{R}=\mathrm{TMS}$ 3c $\mathrm{Ar}=3,5-\left(\mathrm{CH}_{3}\right)_{2} \mathrm{C}_{6} \mathrm{H}_{3}, \mathrm{R}=\mathrm{TBS}$

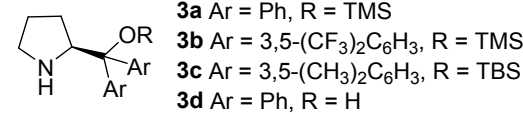<smiles>O=C(O)C1CCCN1</smiles><smiles>CN(C)[C@@H]1CCCC[C@H]1NC(=S)Nc1cc(C(F)(F)F)cc(C(F)(F)F)c1</smiles>

Table S1: Optimization of the Reaction Catalyst, Solvent and Additive

\begin{tabular}{|c|c|c|c|c|c|c|c|}
\hline entry ${ }^{[a]}$ & catalyst & solvent & additive & $t(\mathbf{h})^{[b]}$ & $\begin{array}{l}\text { yield } \\
(\%)^{[c]}\end{array}$ & $\begin{array}{c}\text { ee } \\
(\%)^{[d]}\end{array}$ & $\begin{array}{c}d r \\
(\%)^{[e]}\end{array}$ \\
\hline 1 & $3 a$ & DCE & $\mathrm{BA}$ & 22 & 27 & $>99$ & $>20: 1$ \\
\hline 2 & $3 \mathbf{b}$ & DCE & BA & - & - & - & \\
\hline 3 & $3 c$ & DCE & BA & $>48$ & 6 & $>99$ & $>20: 1$ \\
\hline 4 & $3 d$ & DCE & BA & - & - & - & \\
\hline 5 & $3 \mathbf{e}$ & DCE & - & - & - & - & \\
\hline $6^{[f]}$ & $3 a$ & DCE & BA & 27 & 5 & $>99$ & $>20: 1$ \\
\hline 7 & $3 a$ & toluene & BA & 12 & 20 & $>99$ & $>20: 1$ \\
\hline 8 & $3 a$ & THF & BA & 24 & 25 & $>99$ & $>20: 1$ \\
\hline 9 & $3 \mathbf{a}$ & EA & BA & $>48$ & 26 & 97 & $>20: 1$ \\
\hline 10 & $3 a$ & $\mathrm{CH}_{3} \mathrm{CN}$ & BA & $>48$ & 30 & $>99$ & $>20: 1$ \\
\hline 11 & $3 a$ & 2-Me-THF & BA & $>48$ & 26 & 99 & $>20: 1$ \\
\hline 12 & $3 a$ & $\mathrm{CHCl}_{3}$ & BA & 24 & 30 & $>99$ & $>20: 1$ \\
\hline 13 & $3 a$ & 2-methylfuran & BA & 8 & 9 & $>99$ & $>20: 1$ \\
\hline $14^{[g]}$ & $3 a$ & 2-methylfuran & BA & 14 & 21 & $>99$ & $>20: 1$ \\
\hline 15 & $3 a$ & acetone & BA & 21 & 17 & $>99$ & $>20: 1$ \\
\hline 16 & $3 a$ & MTBE & BA & 10 & 42 & $>99$ & $>20: 1$ \\
\hline 17 & $3 a$ & CPME & BA & 20 & 54 & $>99$ & $>20: 1$ \\
\hline 18 & $3 a$ & CPME & $m$-ClBA & 11 & 54 & $>99$ & $>20: 1$ \\
\hline 19 & $3 a$ & CPME & $p-\mathrm{NO}_{2} \mathrm{BA}$ & 11 & 42 & $>99$ & $>20: 1$ \\
\hline 20 & $3 a$ & CPME & $o$-FBA & 11 & 43 & $>99$ & $>20: 1$ \\
\hline 21 & $3 a$ & CPME & $\mathrm{AcOH}$ & 9 & 53 & $>99$ & $>20: 1$ \\
\hline 22 & $3 a$ & CPME & Pivalic acid & 11 & 45 & $>99$ & $>20: 1$ \\
\hline 23 & $3 a$ & CPME & $\mathrm{NaOAc}$ & $>24$ & - & - & \\
\hline 24 & $3 a$ & CPME & $p$-nitrophenol & $>24$ & 13 & $>99$ & $>20: 1$ \\
\hline
\end{tabular}


[a] Unless otherwise specified, all reactions were carried out in solvent (0.2 $\mathrm{mL})$ with cat. (20 mol \%), additive $(20 \mathrm{~mol} \%)$ at $40{ }^{\circ} \mathrm{C}$ in oil bath. After workup, the mixture was purified by flash chromatography on silica gel to afford 5a. Compound 5a was respectively dissolved in $\mathrm{CH}_{2} \mathrm{Cl}_{2}(0.1 \mathrm{mmol}$ in $0.5 \mathrm{~mL})$ at $0{ }^{\circ} \mathrm{C}$. TEA (6.0 equiv.), $\mathrm{MsCl}$ (2.0 equiv.) and DMAP (0.2 equiv.) were added in order, then transferred to $25^{\circ} \mathrm{C}$. After full conversion of the second step, the residue was purified by flash chromatography on gel to give product 6a. $[b]$ For the first step. [c] Isolated yield of 6a over two steps. [d] Determined by HPLC analyses of isolated compound 6a on chiral stationary phases. [e] Determined by ${ }^{1} \mathrm{H}$ NMR analyses of isolated compound 6a. $[f]$ The reaction was carried out with takemoto $(20 \mathrm{~mol} \%) .[g]$ The reaction was carried out in solvent $(0.4 \mathrm{~mL})$.

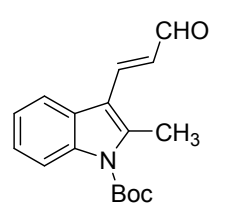

1a<smiles>O=C/C=C/c1ccccc1O</smiles>

2a

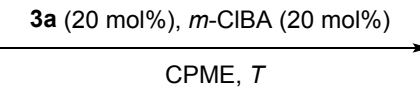

CPME, $T$

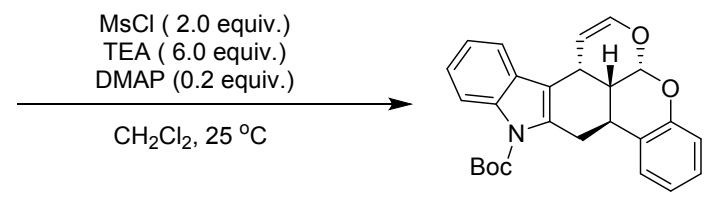

$6 a$

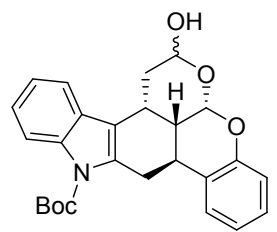

$5 a$

Table S2: Optimization of the Reaction Equivalent and Temperature

\begin{tabular}{|c|c|c|c|c|c|c|c|c|}
\hline entry ${ }^{[a]}$ & $\begin{array}{c}\text { catalys } \\
\mathrm{t} \\
\end{array}$ & $\begin{array}{c}1 a \\
\text { equiv. }\end{array}$ & $\begin{array}{c}2 a \\
\text { equiv. }\end{array}$ & $T\left({ }^{\circ} \mathrm{C}\right)$ & $t(\mathbf{h})^{[b]}$ & $\begin{array}{l}\text { yield } \\
(\%)^{[c]}\end{array}$ & $\begin{array}{c}\text { ee } \\
(\%)^{[d]}\end{array}$ & $\begin{array}{c}d r \\
(\%)^{[e]}\end{array}$ \\
\hline $1^{[f]}$ & $3 a$ & 1.0 & 1.2 & 40 & 11 & 58 & $>99$ & $>20: 1$ \\
\hline 2 & $3 a$ & 1.2 & 1.0 & 40 & 11 & 53 & $>99$ & $>20: 1$ \\
\hline 3 & $3 a$ & 1.5 & 1.0 & 40 & 11 & 31 & $>99$ & $>20: 1$ \\
\hline 4 & $3 a$ & 1.0 & 1.2 & 60 & 23 & 56 & $>99$ & $>20: 1$ \\
\hline
\end{tabular}

[a] Unless otherwise specified, all reactions were carried out in solvent $(0.2 \mathrm{~mL})$. After workup, the mixture was purified by flash chromatography on silica gel to afford $\mathbf{5 a}$. Compound $\mathbf{5 a}$ was respectively dissolved in $\mathrm{CH}_{2} \mathrm{Cl}_{2}(0.1 \mathrm{mmol}$ in $0.5 \mathrm{~mL})$ at $0{ }^{\circ} \mathrm{C}$. TEA (6.0 equiv.), $\mathrm{MsCl}$ (2.0 equiv.) and DMAP (0.2 equiv.) were added in order, then transferred to $25^{\circ} \mathrm{C}$. After full conversion of the second step, the residue was purified by flash chromatography on gel to give product 6a. [b] For the first step. [c] Isolated yield of 6a over two steps. [d] Determined by HPLC analyses of isolated compound $\mathbf{6 a}$ on chiral stationary phases. [e] Determined by ${ }^{1} \mathrm{H}$ NMR analyses of isolated compound 6a. [f] 5a was directly put into the next step without column chromatography separation. 
DCE $=$ dichloroethane

$\mathrm{THF}=$ tetrahydrofuran

$m$-ClBA $=m$-chloro benzoic acid,

$o$-FBA $=o$-fluoro benzoic acid

MTBE $=$ methyl tert-butyl ether

CPME = cyclopentyl methyl ether

2-Me-THF = 2-methyltetrahydrofuran

TEA = triethylamine
$\mathrm{MsCl}=$ methylsufonyl chloride

$\mathrm{EA}=$ ethyl acetate

$\mathrm{BA}=$ benzoic acid

$p-\mathrm{NO}_{2} \mathrm{BA}=p$ - nitrobenzoic acid

TES = triethylsilyl

TMS = trimethylsilyl

TBS = (1,1-dimethylethyl) dimethylsilyl

DMAP = 4-dimethylaminopyridine 


\section{Scope of the reaction}

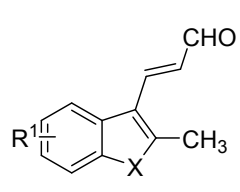

$\mathrm{X}=\mathrm{N}-\mathrm{R}^{3}, \mathrm{~S}$ or $\mathrm{O}$

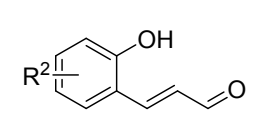

2

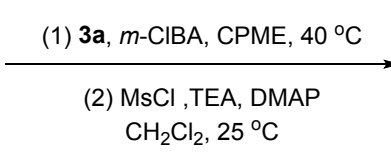

$\mathrm{CH}_{2} \mathrm{Cl}_{2}, 25^{\circ} \mathrm{C}$

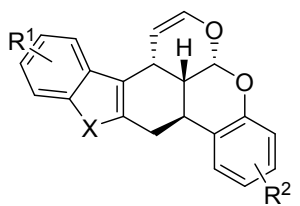

6

General procedure: A glass vial equipped with a magnetic stirring bar was charged with catalyst 3a (0.02 mmol, $6.5 \mathrm{mg}, 20 \mathrm{~mol} \%)$ in CPME (0.2 mL), then 2-hydroxycinnamaldehyde 2 (0.12 mmol, 1.2 equiv.), 3-chlorobenzoic acid (0.02 mmol, $3.1 \mathrm{mg}, 20 \mathrm{~mol} \%)$ and the aldehyde 1 ( $0.1 \mathrm{mmol}, 1.0$ equiv.) was added in one portion and the resulting solution was stirred for about 11 hours at $40{ }^{\circ} \mathrm{C}$ in oil bath until the material 1 disappeared. After completion of the reaction, the crude product was directly put into the next step without column chromatography separation. The crude product (1.0 equiv.) was respectively dissolved in $\mathrm{CH}_{2} \mathrm{Cl}_{2}(0.2 \mathrm{mmol}$ in $1 \mathrm{~mL})$ at $0{ }^{\circ} \mathrm{C}$ in ice bath. TEA (6.0 equiv.), $\mathrm{MsCl}$ (2.0 equiv.), DMAP (0.2 equiv.) were added to the reaction mixtures. After full conversion of the reaction about 8 hours, the reaction mixture was purified by flash chromatography on silica gel (petroleum ether/ethyl acetate $=90 / 1$ to 60/1) to give product 6 for NMR and HPLC analysis.

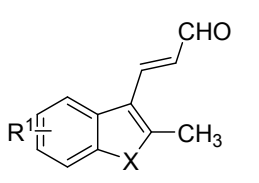

1 $\mathrm{X}=\mathrm{N}-\mathrm{R}^{3}, \mathrm{~S}$ or $\mathrm{O}$<smiles>O=C/C=C/C1=C(O)C=C[R1]C=C1</smiles>

2

$\sqrt{10}$
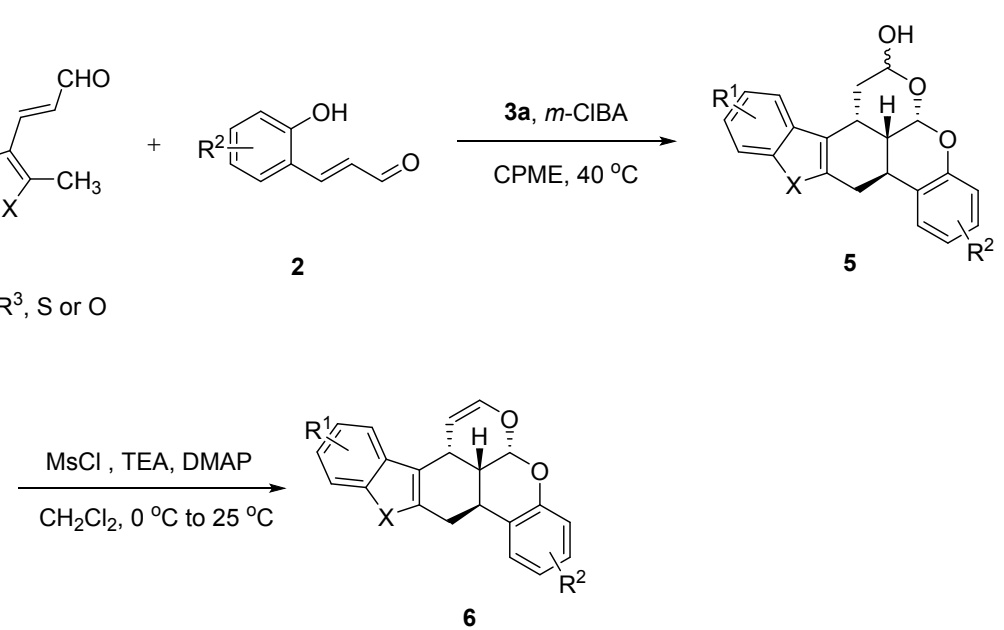

The synthesis of $\mathbf{6 b}, \mathbf{6 d}, \mathbf{6} \mathbf{i}, \mathbf{6 k}$, and $\mathbf{6 r - t}$ had low yield in one-pot method. They were synthesized in two separate steps. The crude product in the first step was purified by 
flash chromatography on silica gel (petroleum ether/ethyl acetate $=5 / 1$ ) to afford $\mathbf{5}$. Then the product 5 (1.0 equiv.) was respectively dissolved in $\mathrm{CH}_{2} \mathrm{Cl}_{2}(0.2 \mathrm{mmol}$ in $1 \mathrm{~mL})$ at $0{ }^{\circ} \mathrm{C}$ in ice bath. TEA (6.0 equiv.), $\mathrm{MsCl}$ (2.0 equiv.), DMAP ( 0.2 equiv.) were added to the reaction mixtures in order. After full conversion of the reaction, the reaction mixture was purified by flash chromatography on silica gel (petroleum ether/ethyl acetate $=$ 90/1 to 60/1) to give product 6 for NMR and HPLC analysis.

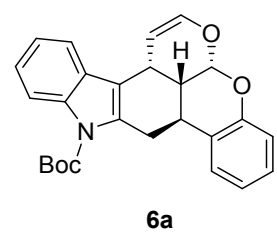

6a was obtained as a white solid $24 \mathrm{mg}$ in $58 \%$ yield for two steps after column chromatography on silica gel (petroleum ether/ethyl acetate $=90 / 1) .{ }^{1} \mathbf{H} \mathbf{~ N M R}(500 \mathrm{MHz}$, $\left.\mathrm{CDCl}_{3}\right) \delta 8.18(\mathrm{~d}, J=7.8 \mathrm{~Hz}, 1 \mathrm{H}), 7.54-7.48(\mathrm{~m}, 1 \mathrm{H}), 7.40(\mathrm{~d}, J=7.7 \mathrm{~Hz}, 1 \mathrm{H}), 7.33-7.29$ (m, 1H), 7.28 (dd, $J=7.4,1.2 \mathrm{~Hz}, 1 \mathrm{H}), 7.22(\mathrm{t}, J=7.6 \mathrm{~Hz}, 1 \mathrm{H}), 7.08-7.03(\mathrm{~m}, 1 \mathrm{H}), 7.03-$ $6.99(\mathrm{~m}, 1 \mathrm{H}), 6.21(\mathrm{dd}, J=6.1,2.3 \mathrm{~Hz}, 1 \mathrm{H}), 5.83(\mathrm{~d}, J=1.6 \mathrm{~Hz}, 1 \mathrm{H}), 5.00(\mathrm{dt}, J=6.2,1.6 \mathrm{~Hz}$, 1H), $4.04(\mathrm{dd}, J=17.6,5.5 \mathrm{~Hz}, 2 \mathrm{H}), 3.39(\mathrm{td}, J=11.8,5.5 \mathrm{~Hz}, 1 \mathrm{H}), 2.95-2.85(\mathrm{~m}, 1 \mathrm{H}), 2.37$ (dd, $J=12.5,5.8 \mathrm{~Hz}, 1 \mathrm{H}), 1.73(\mathrm{~s}, 9 \mathrm{H}) \mathrm{ppm} .{ }^{13} \mathrm{C}$ NMR $\left(125 \mathrm{MHz}, \mathrm{CDCl}_{3}\right) \delta 150.8,150.5$, 139.0, 136.3, 134.2, 128.2, 127.9, 126.4, 124.6, 124.0, 122.8, 122.0, 117.5, 117.0, 116.8, 115.9, 102.5, 95.2, 83.8, 34.8, 30.0, 29.9, 28.4, 26.5 ppm. HRMS: $[\mathrm{M}+\mathrm{H}]^{+}$calcd. For $\mathrm{C}_{26} \mathrm{H}_{26} \mathrm{NO}_{4}{ }^{+}$416.1856, found 416.1850. $[\boldsymbol{\alpha}]_{\mathrm{D}}{ }^{20}+209.02\left(c=1.00\right.$ in $\left.\mathrm{CHCl}_{3}\right)$. The enantiomeric excess was determined by HPLC analysis on Daicel Chiralpak ID column $[n$-hexane $/ i-\mathrm{PrOH}=90 / 10,1 \mathrm{~mL} / \mathrm{min}], \lambda=220 \mathrm{~nm}, t_{\text {major }}=7.75 \mathrm{~min}, t_{\text {minor }}=7.41 \mathrm{~min}$, ee $>\mathbf{9 9 \%}$. The diastereomeric ratio was determined by ${ }^{1} \mathrm{H} N \mathrm{NR}, \boldsymbol{d r}>\mathbf{2 0 : 1}$.

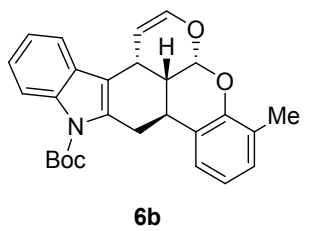

6b was obtained as a white solid $15 \mathrm{mg}$ in $35 \%$ yield for two steps after column chromatography on silica gel (petroleum ether/ethyl acetate $=90 / 1) .{ }^{1} \mathbf{H}$ NMR $(400 \mathrm{MHz}$, 
$\left.\mathrm{CDCl}_{3}\right) \delta 8.17(\mathrm{~d}, J=7.5 \mathrm{~Hz}, 1 \mathrm{H}), 7.54-7.48(\mathrm{~m}, 1 \mathrm{H}), 7.33-7.26(\mathrm{~m}, 2 \mathrm{H}), 7.19(\mathrm{~s}, 1 \mathrm{H})$, $7.02(\mathrm{~d}, J=8.4 \mathrm{~Hz}, 1 \mathrm{H}), 6.90(\mathrm{~d}, J=8.2 \mathrm{~Hz}, 1 \mathrm{H}), 6.20$ (dd, $J=6.1,2.3 \mathrm{~Hz}, 1 \mathrm{H}), 5.81$ (d, $J=$ $1.8 \mathrm{~Hz}, 1 \mathrm{H}), 4.99(\mathrm{dt}, J=6.1,1.6 \mathrm{~Hz}, 1 \mathrm{H}), 4.11-3.95(\mathrm{~m}, 2 \mathrm{H}), 3.35(\mathrm{td}, J=11.7,5.4 \mathrm{~Hz}$, 1H), 2.89 (ddd, $J=17.5,11.2,1.4 \mathrm{~Hz}, 1 \mathrm{H}), 2.42-2.34(\mathrm{~m}, 1 \mathrm{H}), 2.33(\mathrm{~s}, 3 \mathrm{H}), 1.73(\mathrm{~s}, 9 \mathrm{H})$ ppm. ${ }^{13} \mathrm{C}$ NMR $\left(100 \mathrm{MHz}, \mathrm{CDCl}_{3}\right) \delta 150.6,148.6,139.1,136.3,134.4,131.2,128.5,128.3$, $126.8,124.3,124.0,122.8,117.5,116.9,116.7,115.9,102.5,95.3,83.8,34.9,30.0,30.0$, 28.4, 26.5, 20.8 ppm. HRMS: $[\mathrm{M}+\mathrm{H}]^{+}$calcd. For $\mathrm{C}_{27} \mathrm{H}_{28} \mathrm{NO}_{4}{ }^{+} 430.2013$, found 430.2019. $[\alpha]_{\mathbf{D}}{ }^{20}+100.02\left(c=1.25\right.$ in $\left.\mathrm{CHCl}_{3}\right)$. The enantiomeric excess was determined by HPLC analysis on Daicel Chiralpak ID column [ $n$-hexane $/ i$-PrOH $=95 / 5,1 \mathrm{~mL} / \mathrm{min}], \lambda=220$ $\mathrm{nm}, t_{\text {major }}=8.43 \mathrm{~min}, t_{\text {minor }}=9.27 \mathrm{~min}$, ee $>\mathbf{9 9 \%}$. The diastereomeric ratio was determined by ${ }^{1} \mathrm{H}$ NMR, $\boldsymbol{d r}>\mathbf{2 0 : 1}$.

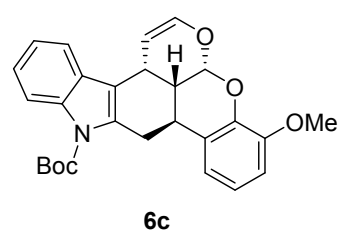

6c was obtained as a white solid $17 \mathrm{mg}$ in $30 \%$ yield for two steps after column chromatography on silica gel (petroleum ether/ethyl acetate $=90 / 1) .{ }^{1} \mathbf{H}$ NMR $(400 \mathrm{MHz}$, $\left.\mathrm{CDCl}_{3}\right) \delta 8.17(\mathrm{~d}, J=7.8 \mathrm{~Hz}, 1 \mathrm{H}), 7.50(\mathrm{~d}, J=7.0 \mathrm{~Hz}, 1 \mathrm{H}), 7.32-7.26(\mathrm{~m}, 2 \mathrm{H}), 7.04-6.97$ (m, 2H), $6.85(\mathrm{dd}, J=6.5,2.7 \mathrm{~Hz}, 1 \mathrm{H}), 6.20(\mathrm{dd}, J=6.1,2.1 \mathrm{~Hz}, 1 \mathrm{H}), 5.94(\mathrm{~d}, J=1.2 \mathrm{~Hz}, 1 \mathrm{H})$, $5.00(\mathrm{~d}, J=6.1 \mathrm{~Hz}, 1 \mathrm{H}), 4.01(\mathrm{dd}, J=17.8,5.3 \mathrm{~Hz}, 2 \mathrm{H}), 3.92(\mathrm{~s}, 3 \mathrm{H}), 3.39(\mathrm{td}, J=11.8,5.5$ $\mathrm{Hz}, 1 \mathrm{H}$ ), 2.90 (dd, $J=17.2,11.2 \mathrm{~Hz}, 1 \mathrm{H}$ ), 2.39 (dd, $J=12.5,5.8 \mathrm{~Hz}, 1 \mathrm{H}$ ), 1.72 (s, 9H) ppm. ${ }^{13}$ C NMR (100 MHz, $\left.\mathrm{CDCl}_{3}\right) \delta 150.5,148.4,140.2,139.0,136.3,134.3,128.2,125.5,124.0$, 122.8, 121.6, 118.1, 117.5, 116.8, 115.9, 110.0 102.5, 95.1, 83.8, 56.0, 34.6, 30.2, 30.0,

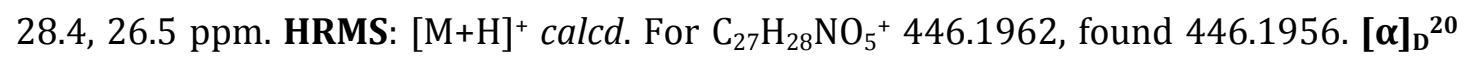
$+104.66\left(c=0.73\right.$ in $\left.\mathrm{CHCl}_{3}\right)$. The enantiomeric excess was determined by HPLC analysis on Daicel Chiralpak IC column [ $n$-hexane/i-PrOH $=90 / 10,1 \mathrm{~mL} / \mathrm{min}], \lambda=205 \mathrm{~nm}, t_{\text {major }}$ $=19.45 \mathrm{~min}, t_{\text {minor }}=13.44 \mathrm{~min}$, ee $\mathbf{> 9 9 \%}$. The diastereomeric ratio was determined by ${ }^{1} \mathrm{H}$ NMR, $\boldsymbol{d r}>\mathbf{2 0 : 1}$. 


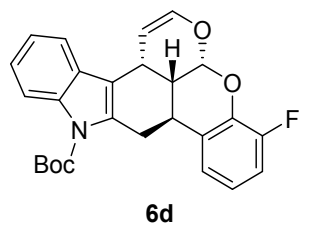

6d was obtained as a white solid $12 \mathrm{mg}$ in $27 \%$ yield for two steps after column chromatography on silica gel (petroleum ether/ethyl acetate $=30 / 1) .{ }^{1} \mathbf{H}$ NMR $(400 \mathrm{MHz}$, $\left.\mathrm{CDCl}_{3}\right) \delta 8.16(\mathrm{dd}, J=7.5,1.5 \mathrm{~Hz}, 1 \mathrm{H}), 7.50(\mathrm{dd}, J=6.8,1.9 \mathrm{~Hz}, 1 \mathrm{H}), 7.34-7.26(\mathrm{~m}, 2 \mathrm{H})$, $7.16(\mathrm{dq}, J=7.6,1.4 \mathrm{~Hz}, 1 \mathrm{H}), 7.06-6.94(\mathrm{~m}, 2 \mathrm{H}), 6.21(\mathrm{dd}, J=6.2,2.4 \mathrm{~Hz}, 1 \mathrm{H}), 5.90(\mathrm{~d}, J=$ $2.0 \mathrm{~Hz}, 1 \mathrm{H}), 5.02(\mathrm{dt}, J=6.1,1.7 \mathrm{~Hz}, 1 \mathrm{H}), 4.07-3.99(\mathrm{~m}, 2 \mathrm{H}), 3.39(\mathrm{td}, J=11.8,5.6 \mathrm{~Hz}$, 1H), 2.90 (ddd, $J=17.6,11.2,1.8 \mathrm{~Hz}, 1 \mathrm{H}$ ), 2.39 (ddt, $J=12.5,5.9,1.8 \mathrm{~Hz}, 1 \mathrm{H}$ ), 1.72 (s, 9H) ppm. ${ }^{13} \mathrm{C}$ NMR $\left(100 \mathrm{MHz}, \mathrm{CDCl}_{3}\right) \delta 150.5,138.9,136.3,134.0,128.2,127.3,124.1,122.9$, $121.5,121.4,121.4,121.3,117.5,116.7,115.9,114.7,114.5,102.7,94.9,83.9,34.6,30.0$, 29.9, 28.4, 26.5 ppm. HRMS: $[\mathrm{M}+\mathrm{H}]^{+}$calcd. For $\mathrm{C}_{26} \mathrm{H}_{25} \mathrm{FNO}_{4}{ }^{+} 434.1689$, found 434.1680. $[\alpha]_{\mathrm{D}}{ }^{20}+101.53\left(c=0.96\right.$ in $\left.\mathrm{CHCl}_{3}\right)$. The enantiomeric excess was determined by HPLC analysis on Daicel Chiralpak IC column [ $n$-hexane $/ i$-PrOH $=85 / 15,1 \mathrm{~mL} / \mathrm{min}], \lambda=220$ $\mathrm{nm}, t_{\text {major }}=9.49 \mathrm{~min}, t_{\text {minor }}=15.33 \mathrm{~min}$, ee $>\mathbf{9 9 \%}$. The diastereomeric ratio was determined by ${ }^{1} \mathrm{H}$ NMR, $\boldsymbol{d r}>\mathbf{2 0 : 1}$.

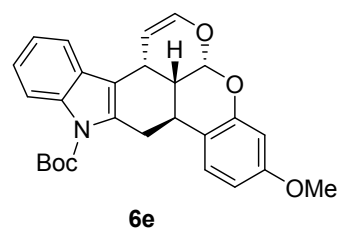

6e was obtained as a white solid $16 \mathrm{mg}$ in $37 \%$ yield for two steps after column chromatography on silica gel (petroleum ether/ethyl acetate $=60 / 1) .{ }^{1} \mathbf{H}$ NMR $(400 \mathrm{MHz}$, $\left.\mathrm{CDCl}_{3}\right) \delta 8.17(\mathrm{~d}, J=7.6 \mathrm{~Hz}, 1 \mathrm{H}), 7.52-7.48(\mathrm{~m}, 1 \mathrm{H}), 7.33-7.27(\mathrm{~m}, 2 \mathrm{H}), 7.24(\mathrm{~s}, 1 \mathrm{H})$, $6.63(\mathrm{dd}, J=8.5,2.6 \mathrm{~Hz}, 1 \mathrm{H}), 6.57(\mathrm{~d}, J=2.6 \mathrm{~Hz}, 1 \mathrm{H}), 6.21(\mathrm{dd}, J=6.1,2.3 \mathrm{~Hz}, 1 \mathrm{H}), 5.81(\mathrm{~d}$, $J=1.7 \mathrm{~Hz}, 1 \mathrm{H}$ ), $5.00(\mathrm{~d}, J=6.1 \mathrm{~Hz}, 1 \mathrm{H}), 3.99$ (dd, $J=17.4,5.6 \mathrm{~Hz}, 2 \mathrm{H}), 3.80$ (s, 3H), 3.31 (td, $J=11.7,5.5 \mathrm{~Hz}, 1 \mathrm{H}), 2.86(\mathrm{dd}, J=16.2,11.2 \mathrm{~Hz}, 1 \mathrm{H}), 2.34(\mathrm{dd}, J=12.5,5.9 \mathrm{~Hz}, 1 \mathrm{H})$, 1.72 (s, 9H) ppm. ${ }^{13} \mathrm{C}$ NMR $\left(100 \mathrm{MHz}, \mathrm{CDCl}_{3}\right) \delta 159.5,151.7,150.5,139.1,136.3,134.3$, 128.3, 127.0, 124.0, 122.8, 117.5, 116.8, 115.9, 108.8, 102.6, 101.9, 95.4, 83.8, 55.4, 35.1, 30.2, 29.9, 28.4, 26.0 ppm. HRMS: $[\mathrm{M}+\mathrm{H}]^{+}$calcd. For $\mathrm{C}_{27} \mathrm{H}_{28} \mathrm{NO}_{5}{ }^{+}$446.1962, found 
446.1958. $[\alpha]_{\mathrm{D}}{ }^{20}+98.90\left(c=1.37\right.$ in $\left.\mathrm{CHCl}_{3}\right)$. The enantiomeric excess was determined by HPLC analysis on Daicel Chiralpak IB column [ $n$-hexane $/ i-\mathrm{PrOH}=90 / 10,1 \mathrm{~mL} / \mathrm{min}], \lambda=$ $205 \mathrm{~nm}, t_{\text {major }}=7.13 \mathrm{~min}, t_{\text {minor }}=8.64 \mathrm{~min}$, ee $>\mathbf{9 9 \%}$. The diastereomeric ratio was determined by ${ }^{1} \mathrm{H}$ NMR, $\boldsymbol{d} \boldsymbol{r}>\mathbf{2 0 : 1}$.

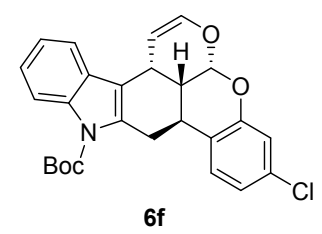

6f was obtained as a white solid $13 \mathrm{mg}$ in $30 \%$ yield for two steps after column chromatography on silica gel (petroleum ether/ethyl acetate $=90 / 1) .{ }^{\mathbf{1}} \mathbf{H} \mathbf{~ N M R}(400 \mathrm{MHz}$, $\left.\mathrm{CDCl}_{3}\right) \delta 8.15(\mathrm{dd}, J=7.4,1.4 \mathrm{~Hz}, 1 \mathrm{H}), 7.50(\mathrm{dd}, J=6.8,1.8 \mathrm{~Hz}, 1 \mathrm{H}), 7.02(\mathrm{dq}, J=3.8,2.1$ $\mathrm{Hz}, 2 \mathrm{H}), 6.20(\mathrm{dd}, J=6.1,2.4 \mathrm{~Hz}, 1 \mathrm{H}), 5.81(\mathrm{~d}, J=2.0 \mathrm{~Hz}, 1 \mathrm{H}), 5.01(\mathrm{dt}, J=6.2,1.7 \mathrm{~Hz}, 1 \mathrm{H})$, $4.06-3.95$ (m, 2H), 3.32 (td, $J=11.5,5.3 \mathrm{~Hz}, 1 \mathrm{H}), 2.87$ (ddd, $J=17.6,11.2,1.8 \mathrm{~Hz}, 1 \mathrm{H}$ ), $2.34(\mathrm{ddt}, J=12.5,5.9,1.8 \mathrm{~Hz}, 1 \mathrm{H}), 1.72(\mathrm{~s}, 9 \mathrm{H}) \mathrm{ppm} .{ }^{13} \mathbf{C} \mathbf{N M R}\left(100 \mathrm{MHz}, \mathrm{CDCl}_{3}\right) \delta 151.5$, 150.5, 138.9, 136.2, 134.0, 133.1, 128.2, 127.5, 124.1, 123.3, 122.9, 122.2, 117.5, 117.2, 116.7, 115.9, 102.6, 95.2, 84.0, 34.6, 29.9, 29.8, 28.4, 26.3 ppm. HRMS: $[\mathrm{M}+\mathrm{H}]^{+}$calcd. For $\mathrm{C}_{26} \mathrm{H}_{25} \mathrm{ClNO}_{4}{ }^{+}$450.1467, found 450.1462. $[\alpha]_{\mathrm{D}}{ }^{20}+88.74\left(c=1.12\right.$ in $\left.\mathrm{CHCl}_{3}\right)$. The enantiomeric excess was determined by HPLC analysis on Daicel Chiralpak ID column $[n$-hexane $/ \mathrm{i}-\mathrm{PrOH}=90 / 10,1 \mathrm{~mL} / \mathrm{min}], \lambda=220 \mathrm{~nm}, t_{\text {major }}=6.53 \mathrm{~min}, t_{\text {minor }}=7.77 \mathrm{~min}$, ee $>\mathbf{9 9 \%}$. The diastereomeric ratio was determined by ${ }^{1} \mathrm{H}$ NMR, $\boldsymbol{d r}>\mathbf{2 0 : 1}$.

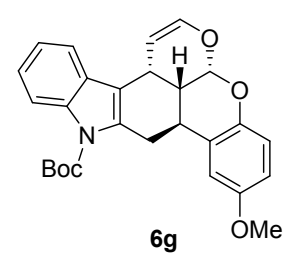

6g was obtained as a white solid $14 \mathrm{mg}$ in $32 \%$ yield for two steps after column chromatography on silica gel (petroleum ether/ethyl acetate $=60 / 1) .{ }^{\mathbf{1}} \mathbf{H} \mathbf{~ N M R}(400 \mathrm{MHz}$, $\left.\mathrm{CDCl}_{3}\right) \delta 8.20(\mathrm{~d}, J=7.4 \mathrm{~Hz}, 1 \mathrm{H}), 7.54-7.47(\mathrm{~m}, 1 \mathrm{H}), 7.35-7.26(\mathrm{~m}, 3 \mathrm{H}), 6.98-6.89(\mathrm{~m}$, 2H), $6.78(\mathrm{ddd}, J=8.9,3.0,1.0 \mathrm{~Hz}, 1 \mathrm{H}), 6.21(\mathrm{dd}, J=6.2,2.4 \mathrm{~Hz}, 1 \mathrm{H}), 5.79(\mathrm{~d}, J=2.0 \mathrm{~Hz}$, 1H), $4.99(\mathrm{dt}, J=6.2,1.7 \mathrm{~Hz}, 1 \mathrm{H}), 4.04-3.93(\mathrm{~m}, 2 \mathrm{H}), 3.80(\mathrm{~s}, 3 \mathrm{H}), 3.36(\mathrm{td}, J=11.8,5.5$ Hz, 1H), 2.89 (ddd, $J=17.5,11.2,1.8 \mathrm{~Hz}, 1 \mathrm{H}$ ), 2.35 (ddt, $J=12.5,6.0,1.8 \mathrm{~Hz}, 1 \mathrm{H}$ ), 1.72 (s, 
9H) ppm. ${ }^{13} \mathrm{C}$ NMR $\left(100 \mathrm{MHz}, \mathrm{CDCl}_{3}\right) \delta 154.7,150.4,144.8,139.1,136.4,134.1,128.2$, $125.36,124.1,122.8,117.5,117.5,116.9,115.9,113.4,111.7,102.5,95.3,83.8,55.8,34.8$, 30.1, 30.0, 28.4, 26.8 ppm. HRMS: $[\mathrm{M}+\mathrm{H}]^{+}$calcd. For $\mathrm{C}_{27} \mathrm{H}_{28} \mathrm{NO}_{5}{ }^{+}$446.1962, found 446.1962. $[\alpha]_{\mathbf{D}}{ }^{20}+92.06\left(c=1.75\right.$ in $\left.\mathrm{CHCl}_{3}\right)$. The enantiomeric excess was determined by HPLC analysis on Daicel Chiralpak IC column [ $n$-hexane $/ i-\mathrm{PrOH}=90 / 10,1 \mathrm{~mL} / \mathrm{min}], \lambda=$ $205 \mathrm{~nm}, t_{\text {major }}=13.67 \mathrm{~min}, t_{\text {minor }}=13.03 \mathrm{~min}$, ee $>\mathbf{9 9 \%}$. The diastereomeric ratio was determined by ${ }^{1} \mathrm{H}$ NMR, $\boldsymbol{d} \boldsymbol{r}>\mathbf{2 0 : 1}$.

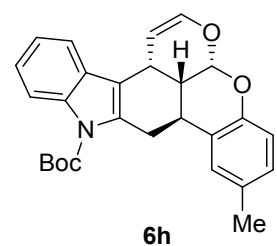

6h was obtained as a white solid 15 mg in 34\% yield for two steps after column chromatography on silica gel (petroleum ether/ethyl acetate $=90 / 1) .{ }^{1} \mathbf{H}$ NMR $(400 \mathrm{MHz}$ $\left.\mathrm{CDCl}_{3}\right) \delta 8.20-8.15(\mathrm{~m}, 1 \mathrm{H}), 7.50(\mathrm{dd}, J=7.7,1.5 \mathrm{~Hz}, 1 \mathrm{H}), 7.34-7.28(\mathrm{~m}, 2 \mathrm{H}), 7.21-7.17$ (m, 1H), 7.02 (dd, $J=8.2,2.0 \mathrm{~Hz}, 1 \mathrm{H}), 6.90(\mathrm{~d}, J=8.3 \mathrm{~Hz}, 1 \mathrm{H}), 6.20(\mathrm{dd}, J=6.1,2.4 \mathrm{~Hz}, 1 \mathrm{H}$ ), $5.80(\mathrm{~d}, J=2.1 \mathrm{~Hz}, 1 \mathrm{H}), 4.99(\mathrm{dt}, J=6.2,1.7 \mathrm{~Hz}, 1 \mathrm{H}), 4.07-3.98(\mathrm{~m}, 2 \mathrm{H}), 3.35(\mathrm{td}, J=11.8$, $5.5 \mathrm{~Hz}, 1 \mathrm{H}$ ), 2.89 (ddd, $J=17.6,11.2,1.8 \mathrm{~Hz}, 1 \mathrm{H}), 2.34(\mathrm{~s}, 4 \mathrm{H}), 1.74(\mathrm{~s}, 9 \mathrm{H}) \quad \mathrm{ppm} .{ }^{13} \mathrm{C}$ NMR $\left(100 \mathrm{MHz}, \mathrm{CDCl}_{3}\right) \delta 150.6,148.6,139.1,136.3,134.4,131.2,128.5,128.3,126.8$, $124.3,124.0,122.8,117.5,116.9,116.7,115.9,102.5,95.3,83.8,34.9,30.0,30.0,28.4$, 26.5, 20.9 ppm. HRMS: $[\mathrm{M}+\mathrm{H}]^{+}$calcd. For $\mathrm{C}_{27} \mathrm{H}_{28} \mathrm{NO}_{4}{ }^{+} 430.2013$, found 430.2011. [ $\left.\alpha\right]_{\mathbf{D}}{ }^{20}$ $+105.86\left(c=1.21\right.$ in $\mathrm{CHCl}_{3}$ ). The enantiomeric excess was determined by HPLC analysis on Daicel Chiralpak IB column $[n$-hexane $/ i$-PrOH $=90 / 10,1 \mathrm{~mL} / \mathrm{min}], \lambda=205 \mathrm{~nm}, t_{\text {major }}$ $=4.99 \mathrm{~min}, t_{\text {minor }}=6.64 \mathrm{~min}$, ee $>\mathbf{9 9 \%}$. The diastereomeric ratio was determined by ${ }^{1} \mathrm{H}$ NMR, $d r>20: 1$.

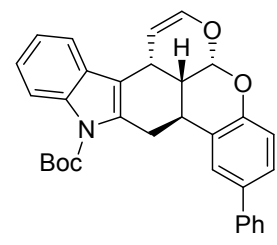

$6 \mathbf{i}$

6i was obtained as a white solid $13 \mathrm{mg}$ in $26 \%$ yield for two steps after column chromatography on silica gel (petroleum ether/ethyl acetate $=90 / 1) .{ }^{1} \mathbf{H}$ NMR $(400 \mathrm{MHz}$, 
$\left.\mathrm{CDCl}_{3}\right) \delta 8.25-8.20(\mathrm{~m}, 1 \mathrm{H}), 7.61-7.54(\mathrm{~m}, 3 \mathrm{H}), 7.54-7.50(\mathrm{~m}, 1 \mathrm{H}), 7.47-7.41(\mathrm{~m}, 3 \mathrm{H})$, $7.38-7.27$ (m, 3H), 7.09 (d, $J=8.4 \mathrm{~Hz}, 1 \mathrm{H}), 6.23$ (dd, $J=6.2,2.4 \mathrm{~Hz}, 1 \mathrm{H}), 5.86$ (d, $J=2.0$ $\mathrm{Hz}, 1 \mathrm{H}), 5.02(\mathrm{dt}, J=6.2,1.8 \mathrm{~Hz}, 1 \mathrm{H}), 4.11(\mathrm{dd}, J=17.5,5.6 \mathrm{~Hz}, 1 \mathrm{H}), 4.04(\mathrm{dp}, J=6.5,2.2$ $\mathrm{Hz}, 1 \mathrm{H}), 3.45(\mathrm{td}, J=11.8,5.6 \mathrm{~Hz}, 1 \mathrm{H}), 2.97(\mathrm{ddd}, J=17.5,11.1,1.7 \mathrm{~Hz}, 1 \mathrm{H}), 2.40(\mathrm{ddt}, J=$ 12.5, 5.9, $1.8 \mathrm{~Hz}, 1 \mathrm{H}), 1.73$ (s, 9H) ppm. ${ }^{13} \mathrm{C}$ NMR (100 MHz, $\left.\mathrm{CDCl}_{3}\right) \delta 150.5,150.4,141.1$, 139.1, 136.4, 135.4, 134.1, 128.8, 128.2, 127.0, 126.9, 126.9, 125.3, 125.0, 124.1, 122.9, $117.5,117.4,116.8,116.0,102.7,95.4,83.9,34.8,30.1,30.0,28.4,26.6$ ppm. HRMS: $[\mathrm{M}+\mathrm{H}]^{+}$calcd. $\mathrm{C}_{32} \mathrm{H}_{30} \mathrm{NO}_{4}{ }^{+}$492.2169, found 492.2166. $[\alpha]_{\mathrm{D}}{ }^{20}+198.09\left(c=1.08\right.$ in $\left.\mathrm{CHCl}_{3}\right)$. The enantiomeric excess was determined by HPLC analysis on Daicel Chiralpak IB column $[n$-hexane $/ i-\mathrm{PrOH}=90 / 10,1 \mathrm{~mL} / \mathrm{min}], \lambda=205 \mathrm{~nm}, t_{\text {major }}=5.92 \mathrm{~min}, t_{\text {minor }}=7.06$ min, ee $>\mathbf{9 9 \%}$. The diastereomeric ratio was determined by ${ }^{1} \mathrm{H} N \mathrm{NR}, \boldsymbol{d r}>\mathbf{2 0 : 1}$.

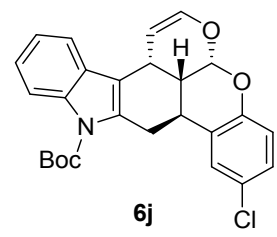

6j was obtained as a white solid $15 \mathrm{mg}$ in $34 \%$ yield for two steps after column chromatography on silica gel (petroleum ether/ethyl acetate $=90 / 1) .{ }^{1} \mathbf{H}$ NMR $(400 \mathrm{MHz}$, $\left.\mathrm{CDCl}_{3}\right) \delta 8.15(\mathrm{~d}, J=7.5 \mathrm{~Hz}, 1 \mathrm{H}), 7.53-7.47(\mathrm{~m}, 1 \mathrm{H}), 7.33-7.26(\mathrm{~m}, 3 \mathrm{H}), 7.01$ (dq, $J=3.7$, $2.1 \mathrm{~Hz}, 2 \mathrm{H}), 6.19$ (dd, $J=6.1,2.4 \mathrm{~Hz}, 1 \mathrm{H}), 5.80$ (d, $J=1.9 \mathrm{~Hz}, 1 \mathrm{H}), 5.00(\mathrm{dt}, J=6.2,1.7 \mathrm{~Hz}$, $1 \mathrm{H}), 4.00(\mathrm{dt}, J=13.6,5.5 \mathrm{~Hz}, 2 \mathrm{H}), 3.31(\mathrm{td}, J=11.6,5.6 \mathrm{~Hz}, 1 \mathrm{H}), 2.86(\mathrm{ddd}, J=17.4,11.2$, $1.5 \mathrm{~Hz}, 1 \mathrm{H}$ ), 2.32 (ddd, $J=12.5,4.3,1.6 \mathrm{~Hz}, 1 \mathrm{H}), 1.71$ (s, 9H) ppm. ${ }^{13} \mathrm{C}$ NMR (100 MHz, $\left.\mathrm{CDCl}_{3}\right) \delta 151.5,150.5,138.9,136.2,134.0,133.1,128.6,127.5,124.1,123.3,122.8,122.2$, 117.5, 117.2, 116.7, 115.9, 102.6, 95.2, 84.0, 34.6, 29.9, 29.8, 28.4, 26.3 ppm. HRMS: $[\mathrm{M}+\mathrm{H}]^{+}$calcd. For $\mathrm{C}_{26} \mathrm{H}_{25} \mathrm{ClNO}_{4}{ }^{+} 450.1467$, found 450.1270. $[\alpha]_{\mathrm{D}}{ }^{20}+85.98(c=1.28$ in $\mathrm{CHCl}_{3}$ ). The enantiomeric excess was determined by HPLC analysis on Daicel Chiralpak IC column $[n$-hexane $/ i-\mathrm{PrOH}=90 / 10,1 \mathrm{~mL} / \mathrm{min}], \lambda=220 \mathrm{~nm}, t_{\text {major }}=6.44 \mathrm{~min}, t_{\text {minor }}=$ $7.11 \mathrm{~min}$, ee $>\mathbf{9 9 \%}$. The diastereomeric ratio was determined by ${ }^{1} \mathrm{H} N \mathrm{NR}, \boldsymbol{d r}>\mathbf{2 0 : 1}$. 


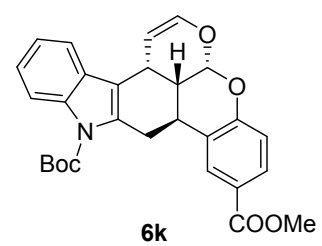

6k was obtained as a white solid $16 \mathrm{mg}$ in $26 \%$ yield for two steps after column chromatography on silica gel (petroleum ether/ethyl acetate $=60 / 1) .{ }^{\mathbf{1}} \mathbf{H}$ NMR $(400 \mathrm{MHz}$, $\left.\mathrm{CDCl}_{3}\right) \delta 8.23(\mathrm{~d}, J=7.9 \mathrm{~Hz}, 1 \mathrm{H}), 8.12(\mathrm{~s}, 1 \mathrm{H}), 7.91(\mathrm{~d}, J=8.5 \mathrm{~Hz}, 1 \mathrm{H}), 7.50(\mathrm{~d}, J=7.7 \mathrm{~Hz}$, 1H), $7.34-7.26(\mathrm{~m}, 2 \mathrm{H}), 7.04(\mathrm{~d}, J=8.5 \mathrm{~Hz}, 1 \mathrm{H}), 6.21(\mathrm{dd}, J=6.1,2.1 \mathrm{~Hz}, 1 \mathrm{H}), 5.86(\mathrm{~s}, 1 \mathrm{H})$, $5.03(\mathrm{~d}, J=6.1 \mathrm{~Hz}, 1 \mathrm{H}), 4.12-4.01(\mathrm{~m}, 2 \mathrm{H}), 3.91(\mathrm{~s}, 3 \mathrm{H}), 3.38(\mathrm{td}, J=11.7,5.5 \mathrm{~Hz}, 1 \mathrm{H})$, $2.91(\mathrm{dd}, J=17.4,11.1 \mathrm{~Hz}, 1 \mathrm{H}), 2.35(\mathrm{dd}, J=12.5,5.8 \mathrm{~Hz}, 1 \mathrm{H}), 1.75(\mathrm{~s}, 9 \mathrm{H}) \mathrm{ppm} .{ }^{13} \mathbf{C} \mathbf{~ N M R}$ $\left(100 \mathrm{MHz}, \mathrm{CDCl}_{3}\right) \delta 166.7,154.8,150.4,138.8,136.4,133.7,129.8,128.6,128.1,124.8$, 124.2, 124.0, 122.8, 117.4, 117.0, 116.5, 115.9, 102.8, 95.3, 84.0, 52.0, 34.5, 30.0, 29.8,

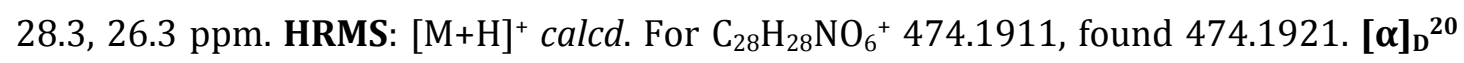
$+202.48\left(c=1.95\right.$ in $\left.\mathrm{CHCl}_{3}\right)$. The enantiomeric excess was determined by HPLC analysis on Daicel Chiralpak IC column [ $n$-hexane $/ i$-PrOH $=90 / 10,1 \mathrm{~mL} / \mathrm{min}], \lambda=205 \mathrm{~nm}, t_{\text {major }}$ $=18.16 \mathrm{~min}, t_{\text {minor }}=15.93 \mathrm{~min}$, ee $\mathbf{9 9 9 \%}$. The diastereomeric ratio was determined by ${ }^{1} \mathrm{H}$ NMR, $\boldsymbol{d} \boldsymbol{r}>\mathbf{2 0 : 1}$.

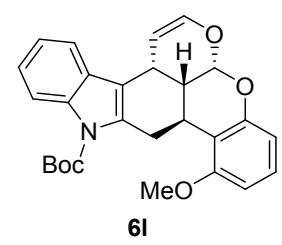

61 was obtained as a white solid $18 \mathrm{mg}$ in $40 \%$ yield for two steps after column chromatography on silica gel (petroleum ether/ethyl acetate $=60 / 1) .{ }^{\mathbf{1}} \mathbf{H}$ NMR $(400 \mathrm{MHz}$, $\left.\mathrm{CDCl}_{3}\right) \delta 8.26-8.19(\mathrm{~m}, 1 \mathrm{H}), 7.49(\mathrm{dd}, J=7.0,1.8 \mathrm{~Hz}, 1 \mathrm{H}), 7.33-7.26(\mathrm{~m}, 2 \mathrm{H}), 7.17(\mathrm{t}, J=$ $8.2 \mathrm{~Hz}, 1 \mathrm{H}), 6.67$ (dd, J = 8.2, $1.1 \mathrm{~Hz}, 1 \mathrm{H}), 6.61-6.54(\mathrm{~m}, 1 \mathrm{H}), 6.20(\mathrm{dd}, J=6.2,2.5 \mathrm{~Hz}, 1 \mathrm{H})$, $5.78(\mathrm{~d}, J=2.1 \mathrm{~Hz}, 1 \mathrm{H}), 5.01(\mathrm{dt}, J=6.2,1.8 \mathrm{~Hz}, 1 \mathrm{H}), 4.59(\mathrm{dd}, J=17.9,4.6 \mathrm{~Hz}, 1 \mathrm{H}), 4.00$ (dq, $J=4.3,1.9 \mathrm{~Hz}, 1 \mathrm{H}$ ), 3.87 (s, 3H), 3.34 (td, $J=11.6,4.5 \mathrm{~Hz}, 1 \mathrm{H}$ ), 2.70 (ddd, $J=17.9$, 11.3, $1.9 \mathrm{~Hz}, 1 \mathrm{H}), 2.46(\mathrm{ddt}, J=11.8,5.7,1.8 \mathrm{~Hz}, 1 \mathrm{H}), 1.68(\mathrm{~s}, 9 \mathrm{H}) \mathrm{ppm} .{ }^{13} \mathbf{C}$ NMR (100 $\left.\mathrm{MHz}, \mathrm{CDCl}_{3}\right) \delta 159.7,152.3,150.4,139.6,136.6,136.1,128.3,128.1,123.8,122.7,117.3$, 116.0, 115.7, 113.1, 110.4, 104.5, 102.2, 94.9, 83.4, 55.5, 36.5, 30.8, 30.6, 28.3, 26.8 ppm. 
HRMS: $[\mathrm{M}+\mathrm{H}]^{+}$calcd. For $\mathrm{C}_{27} \mathrm{H}_{28} \mathrm{NO}_{5}{ }^{+} 446.1962$, found 446.1960. $[\alpha]_{\mathbf{D}}{ }^{20}+255.25(c=1.50$ in $\mathrm{CHCl}_{3}$ ). The enantiomeric excess was determined by HPLC analysis on Daicel Chiralpak IB column $[n$-hexane $/ i$-PrOH $=90 / 10,1 \mathrm{~mL} / \mathrm{min}], \lambda=205 \mathrm{~nm}, t_{\text {major }}=5.95 \mathrm{~min}$, $t_{\text {minor }}=5.28 \mathrm{~min}$, ee $\mathbf{> 9 9 \%}$. The diastereomeric ratio was determined by ${ }^{1} \mathrm{H} \mathrm{NMR}$, $d r>20: 1$.

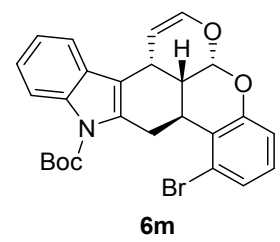

6m was obtained as a white solid $28 \mathrm{mg}$ in $58 \%$ yield for two steps after column chromatography on silica gel (petroleum ether/ethyl acetate $=60 / 1) .{ }^{1} \mathbf{H}$ NMR $(400 \mathrm{MHz}$, $\left.\mathrm{CDCl}_{3}\right) \delta 8.24(\mathrm{dd}, J=7.7,1.4 \mathrm{~Hz}, 1 \mathrm{H}), 7.52-7.48(\mathrm{~m}, 1 \mathrm{H}), 7.33-7.26(\mathrm{~m}, 3 \mathrm{H}), 7.08-7.03$ (m, 1H), $6.98(\mathrm{dd}, J=8.2,1.4 \mathrm{~Hz}, 1 \mathrm{H}), 6.23(\mathrm{dd}, J=6.1,2.5 \mathrm{~Hz}, 1 \mathrm{H}), 5.78(\mathrm{~d}, J=2.1 \mathrm{~Hz}, 1 \mathrm{H})$, $5.11-5.01(\mathrm{~m}, 2 \mathrm{H}), 4.03(\mathrm{dq}, J=4.3,1.9 \mathrm{~Hz}, 1 \mathrm{H}), 3.33(\mathrm{td}, J=11.3,4.1 \mathrm{~Hz}, 1 \mathrm{H}), 2.64$ (ddd, $J=17.5,11.2,1.9 \mathrm{~Hz}, 1 \mathrm{H}), 2.53(\mathrm{ddt}, J=11.4,5.4,1.9 \mathrm{~Hz}, 1 \mathrm{H}), 1.69(\mathrm{~s}, 9 \mathrm{H}) \mathrm{ppm} .{ }^{13} \mathbf{C} \mathbf{~ N M R}$ $\left(100 \mathrm{MHz}, \mathrm{CDCl}_{3}\right) \delta 152.6,150.3,139.9,136.7,135.3,128.8,128.1,128.1,124.2,124.0$, $123.4,122.8,117.3,117.3,116.0,115.8,102.0,94.5,83.9,37.5,31.0,30.6,30.1,28.4 \mathrm{ppm}$ HRMS: $[\mathrm{M}+\mathrm{H}]^{+}$calcd. For $\mathrm{C}_{26} \mathrm{H}_{25} \mathrm{BrNO}_{4}{ }^{+}$494.0961, found 494.0959. $[\alpha]_{\mathrm{D}}{ }^{20}+265.41(c=$ 2.40 in $\mathrm{CHCl}_{3}$ ). The enantiomeric excess was determined by HPLC analysis on Daicel Chiralpak IB column $[n$-hexane $/ i-\mathrm{PrOH}=90 / 10,1 \mathrm{~mL} / \mathrm{min}], \lambda=220 \mathrm{~nm}, t_{\text {major }}=5.23 \mathrm{~min}$, $t_{\text {minor }}=5.81 \mathrm{~min}$, ee $\mathbf{> 9 9 \%}$. The diastereomeric ratio was determined by ${ }^{1} \mathrm{H} \mathrm{NMR}$, $d r>20: 1$.

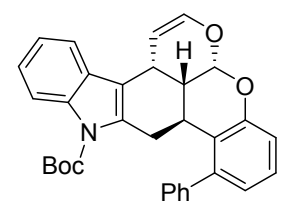

$6 n$

6n was obtained as a white solid $14 \mathrm{mg}$ in $29 \%$ yield for two steps after column chromatography on silica gel (petroleum ether/ethyl acetate $=60 / 1) .{ }^{1} \mathbf{H}$ NMR $(400 \mathrm{MHz}$, $\left.\mathrm{CDCl}_{3}\right) \delta 8.04(\mathrm{dd}, J=6.8,2.6 \mathrm{~Hz}, 1 \mathrm{H}), 7.50-7.28(\mathrm{~m}, 6 \mathrm{H}), 7.26-7.18(\mathrm{~m}, 3 \mathrm{H}), 7.03(\mathrm{dd}, J$ $=8.1,1.4 \mathrm{~Hz}, 1 \mathrm{H}), 6.94(\mathrm{dd}, J=7.5,1.4 \mathrm{~Hz}, 1 \mathrm{H}), 6.31(\mathrm{dd}, J=6.2,2.5 \mathrm{~Hz}, 1 \mathrm{H}), 5.82(\mathrm{~d}, J=$ 
$2.0 \mathrm{~Hz}, 1 \mathrm{H}), 5.05(\mathrm{dt}, J=6.2,1.7 \mathrm{~Hz}, 1 \mathrm{H}), 3.99(\mathrm{dq}, J=6.0,2.1 \mathrm{~Hz}, 1 \mathrm{H}), 3.59(\mathrm{td}, J=11.4$, $4.4 \mathrm{~Hz}, 1 \mathrm{H}), 2.87(\mathrm{dd}, J=17.7,4.4 \mathrm{~Hz}, 1 \mathrm{H}), 2.48-2.42$ (m, 1H), 2.35 (ddd, $J=17.7,11.3$, $1.9 \mathrm{~Hz}, 1 \mathrm{H}), 1.51(\mathrm{~s}, 9 \mathrm{H}) \mathrm{ppm} .{ }^{13} \mathbf{C}$ NMR $\left(100 \mathrm{MHz}, \mathrm{CDCl}_{3}\right) \delta 151.7,149.8,143.2,142.9$, $139.9,136.3,135.7,128.2,128.0,127.7,127.2,125.5,123.8,122.6,121.9,117.4,116.8$, 115.9, 115.4, 102.2, 94.7, 83.5, 37.0, 31.0, 30.9, 28.1, 27.8 ppm. HRMS: $[\mathrm{M}+\mathrm{H}]^{+}$calcd. For $\mathrm{C}_{32} \mathrm{H}_{30} \mathrm{NO}_{4}{ }^{+}$492.2169, found 492.2178. $[\boldsymbol{\alpha}]_{\mathrm{D}}{ }^{20}+255.92\left(c=0.83\right.$ in $\left.\mathrm{CHCl}_{3}\right)$. The enantiomeric excess was determined by HPLC analysis on Daicel Chiralpak IC column $[n$-hexane $/ i-\mathrm{PrOH}=90 / 10,1 \mathrm{~mL} / \mathrm{min}], \lambda=220 \mathrm{~nm}, t_{\text {major }}=7.22 \mathrm{~min}, t_{\text {minor }}=5.73 \mathrm{~min}$, ee $\mathbf{= 9 9 \%}$. The diastereomeric ratio was determined by ${ }^{1} \mathrm{H}$ NMR, $\boldsymbol{d r}>\mathbf{2 0 : 1}$.

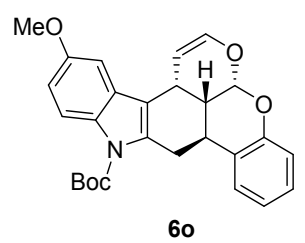

60 was obtained as a white solid $21 \mathrm{mg}$ in $47 \%$ yield for two steps after column chromatography on silica gel (petroleum ether/ethyl acetate $=60 / 1) .{ }^{1} \mathbf{H}$ NMR $(400 \mathrm{MHz}$, $\left.\mathrm{CDCl}_{3}\right) \delta 8.06(\mathrm{~d}, J=9.0 \mathrm{~Hz}, 1 \mathrm{H}), 7.38(\mathrm{~d}, J=7.6 \mathrm{~Hz}, 1 \mathrm{H}), 7.22(\mathrm{t}, J=7.6 \mathrm{~Hz}, 1 \mathrm{H}), 7.07-$ $6.98(\mathrm{~m}, 2 \mathrm{H}), 6.95(\mathrm{~d}, J=2.3 \mathrm{~Hz}, 1 \mathrm{H}), 6.90(\mathrm{dd}, J=9.0,2.4 \mathrm{~Hz}, 1 \mathrm{H}), 6.21$ (dd, $J=6.1,2.2 \mathrm{~Hz}$, 1H), $5.88-5.76(\mathrm{~m}, 1 \mathrm{H}), 4.99(\mathrm{~d}, J=6.1 \mathrm{~Hz}, 1 \mathrm{H}), 4.08-3.94(\mathrm{~m}, 2 \mathrm{H}), 3.88(\mathrm{~s}, 3 \mathrm{H}), 3.37$ (td, $J=11.8,5.5 \mathrm{~Hz}, 1 \mathrm{H}), 2.87(\mathrm{dd}, J=17.4,11.2 \mathrm{~Hz}, 1 \mathrm{H}), 2.34(\mathrm{dd}, J=12.5,5.8 \mathrm{~Hz}, 1 \mathrm{H})$, 1.71 (s, 9H) ppm. ${ }^{13} \mathrm{C}$ NMR $\left(100 \mathrm{MHz}, \mathrm{CDCl}_{3}\right) \delta 156.0,150.8,150.4,139.1,135.0,130.9$, 129.1, 128.0, 126.4, 124.7, 122.0, 117.0, 116.7, 116.6, 111.9, 102.4, 100.7, 95.3, 83.7, 55.8, 34.8, 30.1, 29.9, 28.4, 26.4 ppm. HRMS: $[\mathrm{M}+\mathrm{H}]^{+}$calcd. For $\mathrm{C}_{27} \mathrm{H}_{28} \mathrm{NO}_{5}{ }^{+} 446.1962$, found 446.1956. $[\alpha]_{\mathrm{D}}{ }^{20}+100.61\left(c=1.75\right.$ in $\left.\mathrm{CHCl}_{3}\right)$. The enantiomeric excess was determined by HPLC analysis on Daicel Chiralpak IB column [ $n$-hexane $/ i$-PrOH $=90 / 10,1 \mathrm{~mL} / \mathrm{min}$, $\lambda=205 \mathrm{~nm}, t_{\text {major }}=7.19 \mathrm{~min}, t_{\text {minor }}=8.17 \mathrm{~min}$, ee $>\mathbf{9 9 \%}$. The diastereomeric ratio was determined by ${ }^{1} \mathrm{H}$ NMR, $\boldsymbol{d r}>\mathbf{2 0 : 1}$. 


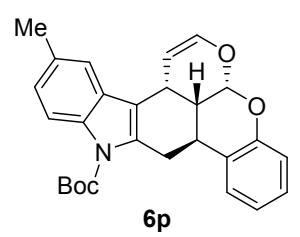

6p was obtained as a white solid $24 \mathrm{mg}$ in $56 \%$ yield for two steps after column chromatography on silica gel (petroleum ether/ethyl acetate $=60 / 1) .{ }^{\mathbf{1}} \mathbf{H} \mathbf{~ N M R}(400 \mathrm{MHz}$, $\left.\mathrm{CDCl}_{3}\right) \delta 8.04(\mathrm{~d}, J=8.5 \mathrm{~Hz}, 1 \mathrm{H}), 7.39(\mathrm{~d}, J=7.7 \mathrm{~Hz}, 1 \mathrm{H}), 7.28(\mathrm{~s}, 1 \mathrm{H}), 7.22(\mathrm{t}, J=7.7 \mathrm{~Hz}$, $1 \mathrm{H}), 7.12(\mathrm{dd}, J=8.5,1.0 \mathrm{~Hz}, 1 \mathrm{H}), 7.07-6.98(\mathrm{~m}, 2 \mathrm{H}), 6.21(\mathrm{dd}, J=6.1,2.3 \mathrm{~Hz}, 1 \mathrm{H}), 5.82$ $(\mathrm{d}, J=1.8 \mathrm{~Hz}, 1 \mathrm{H}), 5.00(\mathrm{dt}, J=6.1,1.6 \mathrm{~Hz}, 1 \mathrm{H}), 4.00(\mathrm{ddd}, J=10.7,5.9,3.7 \mathrm{~Hz}, 2 \mathrm{H}), 3.37$ $(\mathrm{td}, J=11.8,5.5 \mathrm{~Hz}, 1 \mathrm{H}), 2.88(\mathrm{ddd}, J=17.4,11.2,1.3 \mathrm{~Hz}, 1 \mathrm{H}), 2.47$ (s, 3H), 2.34 (dd, $J=$ 12.5, $5.9 \mathrm{~Hz}, 1 \mathrm{H}), 1.72$ (s, 9H) ppm. ${ }^{13} \mathrm{C}$ NMR (100 MHz, $\left.\mathrm{CDCl}_{3}\right) \delta 150.9,150.5,139.0$, 134.5, 134.3, 132.3, 128.4, 127.9, 126.4, 125.3, 124.7, 122.0, 117.5, 117.0, 116.6, 115.6, 102.6, 95.3, 83.7, 34.8, 30.0, 29.9, 28.4, 26.5, 21.4 ppm. HRMS: $[\mathrm{M}+\mathrm{H}]^{+}$calcd. For $\mathrm{C}_{27} \mathrm{H}_{28} \mathrm{NO}_{4}{ }^{+}$430.2013, found 430.2017. $[\alpha]_{\mathbf{D}}{ }^{20}+147.04$ ( $c=1.25$ in $\mathrm{CHCl}_{3}$ ). The enantiomeric excess was determined by HPLC analysis on Daicel Chiralpak AD column $[n$-hexane $/ i-\mathrm{PrOH}=90 / 10,1 \mathrm{~mL} / \mathrm{min}], \lambda=205 \mathrm{~nm}, t_{\text {major }}=4.95 \mathrm{~min}, t_{\text {minor }}=5.44 \mathrm{~min}, \mathbf{e e}$ $\mathbf{= 9 7 \%}$. The diastereomeric ratio was determined by ${ }^{1} \mathrm{H}$ NMR, $\boldsymbol{d r}>\mathbf{2 0 : 1}$.

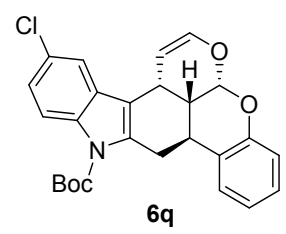

6q was obtained as a white solid $18 \mathrm{mg}$ in $39 \%$ yield for two steps after column chromatography on silica gel (petroleum ether/ethyl acetate $=60 / 1) .{ }^{\mathbf{1}} \mathbf{H}$ NMR $(400 \mathrm{MHz}$, $\left.\mathrm{CDCl}_{3}\right) \delta 8.09(\mathrm{~d}, J=8.9 \mathrm{~Hz}, 1 \mathrm{H}), 7.46(\mathrm{~d}, J=2.1 \mathrm{~Hz}, 1 \mathrm{H}), 7.36(\mathrm{~d}, J=7.7 \mathrm{~Hz}, 1 \mathrm{H}), 7.26-$ 7.19 (m, 2H), $7.08-7.03$ (m, 1H), 7.01 (dd, $J=8.1,1.3 \mathrm{~Hz}, 1 \mathrm{H}), 6.21$ (dd, $J=6.1,2.4 \mathrm{~Hz}$, $1 \mathrm{H}), 5.81(\mathrm{~d}, J=2.0 \mathrm{~Hz}, 1 \mathrm{H}), 4.95(\mathrm{dt}, J=6.3,1.8 \mathrm{~Hz}, 1 \mathrm{H}), 4.01(\mathrm{dd}, J=17.7,5.6 \mathrm{~Hz}, 1 \mathrm{H})$, $3.96(\mathrm{dq}, J=6.1,2.0 \mathrm{~Hz}, 1 \mathrm{H}), 3.37$ (td, $J=11.8,5.5 \mathrm{~Hz}, 1 \mathrm{H}$ ), 2.88 (ddd, $J=17.6,11.2,1.8$ $\mathrm{Hz}, 1 \mathrm{H}), 2.35$ (ddt, $J=12.6,6.0,1.8 \mathrm{~Hz}, 1 \mathrm{H}), 1.72(\mathrm{~s}, 9 \mathrm{H})$ ppm. ${ }^{13} \mathbf{C}$ NMR $\left(100 \mathrm{MHz}, \mathrm{CDCl}_{3}\right)$ $\delta 150.8,150.1,139.2,135.7,134.7,129.5,128.5,128.0,126.3,124.4,124.1,122.1,117.2$, 117.1, 116.9, 116.3, 102.2, 95.1, 84.3, 34.7, 30.1, 29.8, 28.3, 26.4 ppm. HRMS: [M+H] ${ }^{+}$ 
calcd. For $\mathrm{C}_{26} \mathrm{H}_{25} \mathrm{ClNO}_{4}{ }^{+} 450.1467$, found 450.1464 . [ $\left.\alpha\right]_{\mathrm{D}}{ }^{20}+93.15\left(c=1.46\right.$ in $\left.\mathrm{CHCl}_{3}\right)$. The enantiomeric excess was determined by HPLC analysis on Daicel Chiralpak AD column $[n$-hexane $/ i-\mathrm{PrOH}=90 / 10,1 \mathrm{~mL} / \mathrm{min}], \lambda=205 \mathrm{~nm}, t_{\text {major }}=5.33 \mathrm{~min}, t_{\text {minor }}=6.70 \mathrm{~min}$, ee $>\mathbf{9 9 \%}$. The diastereomeric ratio was determined by ${ }^{1} \mathrm{H}$ NMR, $\boldsymbol{d r}>\mathbf{2 0 : 1}$.

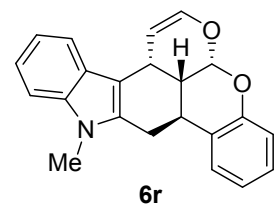

6r was obtained as a yellow solid $11 \mathrm{mg}$ in $32 \%$ yield for two steps after column chromatography on silica gel (petroleum ether/ethyl acetate $=30 / 1) .{ }^{1} \mathbf{H}$ NMR $(400 \mathrm{MHz}$, $\left.\mathrm{CDCl}_{3}\right) \delta 7.58(\mathrm{~d}, J=7.8 \mathrm{~Hz}, 1 \mathrm{H}), 7.39(\mathrm{~d}, J=7.7 \mathrm{~Hz}, 1 \mathrm{H}), 7.33(\mathrm{~d}, J=8.1 \mathrm{~Hz}, 1 \mathrm{H}), 7.23(\mathrm{td}, J$ = 7.0, $1.7 \mathrm{~Hz}, 2 \mathrm{H}), 7.18-7.11(\mathrm{~m}, 1 \mathrm{H}), 7.09-7.00(\mathrm{~m}, 2 \mathrm{H}), 6.19$ (dd, $J=6.1,2.3 \mathrm{~Hz}, 1 \mathrm{H}$ ), $5.84(\mathrm{~d}, J=1.8 \mathrm{~Hz}, 1 \mathrm{H}), 5.05(\mathrm{dt}, J=6.1,1.6 \mathrm{~Hz}, 1 \mathrm{H}), 4.11(\mathrm{dd}, J=4.0,1.8 \mathrm{~Hz}, 1 \mathrm{H}), 3.73(\mathrm{~s}$, 3H), 3.59 (dd, $J=15.5,5.5 \mathrm{~Hz}, 1 \mathrm{H}), 3.49(\mathrm{td}, J=11.7,5.5 \mathrm{~Hz}, 1 \mathrm{H}), 2.76-2.64(\mathrm{~m}, 1 \mathrm{H}), 2.40$ (dd, $J=12.3,5.9 \mathrm{~Hz}, 1 \mathrm{H})$ ppm. ${ }^{13} \mathrm{C}$ NMR $\left(100 \mathrm{MHz}, \mathrm{CDCl}_{3}\right) \delta 151.0,138.3,137.3,134.0$, 128.0, 126.3, 125.9, 124.7, 121.9, 121.3, 119.3, 117.6, 117.1, 109.7, 109.1, 104.4, 95.7, 35.8, 30.1, 29.4, 26.6, 26.3 ppm. HRMS: $[\mathrm{M}+\mathrm{H}]^{+}$calcd. For $\mathrm{C}_{22} \mathrm{H}_{20} \mathrm{NO}_{2}{ }^{+} 330.1489$, found 330.1483. $[\alpha]_{\mathbf{D}}{ }^{20}+38.11\left(c=1.42\right.$ in $\left.\mathrm{CHCl}_{3}\right)$. The enantiomeric excess was determined by HPLC analysis on Daicel Chiralpak ID column [ $n$-hexane $/ i$-PrOH $=90 / 10,1 \mathrm{~mL} / \mathrm{min}], \lambda=$ $205 \mathrm{~nm}, t_{\text {major }}=11.12 \mathrm{~min}, t_{\text {minor }}=13.12 \mathrm{~min}$, ee $>\mathbf{9 9 \%}$. The diastereomeric ratio was determined by ${ }^{1} \mathrm{H}$ NMR, $\boldsymbol{d} \boldsymbol{r}>\mathbf{2 0 : 1}$.

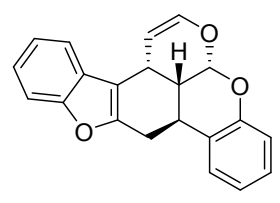

$6 s$

6s was obtained as a white solid $17 \mathrm{mg}$ in $54 \%$ yield for two steps after column chromatography on silica gel (petroleum ether/ethyl acetate $=60 / 1) .{ }^{1} \mathbf{H}$ NMR $(400 \mathrm{MHz}$, $\left.\mathrm{CDCl}_{3}\right) \delta 7.56-7.50(\mathrm{~m}, 1 \mathrm{H}), 7.49-7.45(\mathrm{~m}, 1 \mathrm{H}), 7.34-7.26(\mathrm{~m}, 3 \mathrm{H}), 7.24-7.20(\mathrm{~m}, 1 \mathrm{H})$, $7.09-6.98$ (m, 2H), 6.23 (dd, $J=6.1,2.4 \mathrm{~Hz}, 1 \mathrm{H}), 5.83$ (d, $J=2.1 \mathrm{~Hz}, 1 \mathrm{H}), 4.99$ (dt, $J=6.1$, $1.7 \mathrm{~Hz}, 1 \mathrm{H}), 4.01(\mathrm{dq}, J=6.1,2.1 \mathrm{~Hz}, 1 \mathrm{H}), 3.59$ (dd, $J=16.2,5.6 \mathrm{~Hz}, 1 \mathrm{H}), 3.48(\mathrm{td}, J=11.6$, $5.6 \mathrm{~Hz}, 1 \mathrm{H}), 2.79$ (ddd, $J=16.2,11.0,1.8 \mathrm{~Hz}, 1 \mathrm{H}), 2.41(\mathrm{ddt}, J=12.4,5.9,1.8 \mathrm{~Hz}, 1 \mathrm{H}) \mathrm{ppm}$. 
${ }^{13} \mathrm{C}$ NMR $\left(100 \mathrm{MHz}, \mathrm{CDCl}_{3}\right) \delta 154.9,152.5,150.8,139.2,128.2,127.3,126.3,123.9,123.8$, 122.7, 122.1, 118.3, 117.0, 113.8, 111.3, 102.5, 95.4, 35.5, 29.8, 27.6, 26.6 ppm. HRMS: $[\mathrm{M}+\mathrm{H}]^{+}$calcd. For $\mathrm{C}_{21} \mathrm{H}_{17} \mathrm{O}_{3}{ }^{+}$317.1172, found 317.1173. $[\alpha]_{\mathrm{D}}{ }^{20}+44.18\left(c=1.42\right.$ in $\left.\mathrm{CHCl}_{3}\right)$. The enantiomeric excess was determined by HPLC analysis on Daicel Chiralpak ID column $[n$-hexane $/ i$-PrOH $=90 / 10,1 \mathrm{~mL} / \mathrm{min}], \lambda=205 \mathrm{~nm}, t_{\text {major }}=7.26 \mathrm{~min}, t_{\text {minor }}=$ $11.40 \mathrm{~min}, \mathbf{e e}=\mathbf{9 5 \%}$. The diastereomeric ratio was determined by ${ }^{1} \mathrm{H}$ NMR, $\boldsymbol{d r}>\mathbf{2 0 : 1}$.

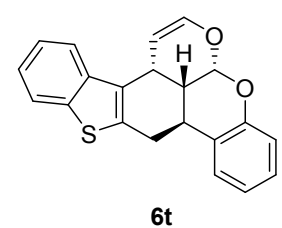

6t was obtained as a white solid $8 \mathrm{mg}$ in $24 \%$ yield for two steps after column chromatography on silica gel (petroleum ether/ethyl acetate $=60 / 1) .{ }^{\mathbf{1}} \mathbf{H} \mathbf{~ N M R}(400 \mathrm{MHz}$, $\left.\mathrm{CDCl}_{3}\right) \delta 7.82(\mathrm{dt}, J=8.0,0.9 \mathrm{~Hz}, 1 \mathrm{H}), 7.70(\mathrm{dt}, J=8.0,1.0 \mathrm{~Hz}, 1 \mathrm{H}), 7.40(\mathrm{td}, J=8.0,7.6,1.2$ $\mathrm{Hz}, 1 \mathrm{H}), 7.33$ (ddd, $J=8.4,5.4,1.7 \mathrm{~Hz}, 2 \mathrm{H}), 7.27-7.19$ (m, 4H), $7.09-6.98$ (m, 2H), 6.23 (dd, $J=6.2,2.4 \mathrm{~Hz}, 1 \mathrm{H}), 5.85(\mathrm{~d}, J=2.0 \mathrm{~Hz}, 1 \mathrm{H}), 5.00(\mathrm{dt}, J=6.2,1.8 \mathrm{~Hz}, 1 \mathrm{H}), 4.13$ (dq, $J=$ 6.0, $2.0 \mathrm{~Hz}, 1 \mathrm{H}$ ), 3.70 (dd, $J=16.3,5.3 \mathrm{~Hz}, 1 \mathrm{H}$ ), 3.50 (td, $J=11.9,5.4 \mathrm{~Hz}, 1 \mathrm{H}$ ), 2.89 (ddd, $J$ $=16.4,11.3,1.5 \mathrm{~Hz}, 1 \mathrm{H}), 2.44(\mathrm{ddt}, J=12.4,5.9,1.8 \mathrm{~Hz}, 1 \mathrm{H}) \mathrm{ppm} .{ }^{13} \mathrm{C}$ NMR $(100 \mathrm{MHz}$, $\left.\mathrm{CDCl}_{3}\right) \delta 150.8,139.4,139.1,138.2,136.3,129.4,128.1,126.3,124.3,124.2,124.2,122.7$, 122.1, 120.5, 117.1, 102.2, 95.3, 35.1, 32.3, 29.8, 26.9 ppm. HRMS: [M+H]+ calcd. For $\mathrm{C}_{21} \mathrm{H}_{17} \mathrm{O}_{2} \mathrm{~S}^{+}$333.0944, found 333.0938. $[\boldsymbol{\alpha}]_{\mathbf{D}}{ }^{20}+48.27$ ( $c=0.67$ in $\left.\mathrm{CHCl}_{3}\right)$. The enantiomeric excess was determined by HPLC analysis on Daicel Chiralpak ID column $[n$-hexane $/ \mathrm{i}-\mathrm{PrOH}=90 / 10,1 \mathrm{~mL} / \mathrm{min}], \lambda=205 \mathrm{~nm}, t_{\text {major }}=7.46 \mathrm{~min}, t_{\text {minor }}=10.05 \mathrm{~min}$, $\mathbf{e e}=\mathbf{9 9} \%$. The diastereomeric ratio was determined by ${ }^{1} \mathrm{H} N \mathrm{NR}, \boldsymbol{d r}>\mathbf{2 0}: \mathbf{1}$. 


\section{E. Other reactions}

\section{E1. Synthesis of 8}

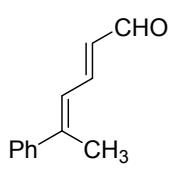

$7 a$

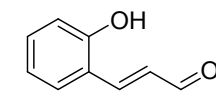

2a

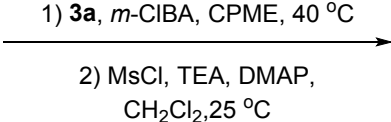

$\mathrm{CH}_{2} \mathrm{Cl}_{2}, 25^{\circ} \mathrm{C}$

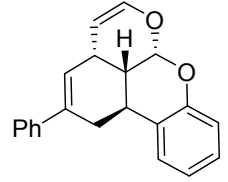

Detailed method: A glass vial equipped with a magnetic stirring bar was charged with catalyst $3 a(0.02 \mathrm{mmol}, 6.5 \mathrm{mg}, 20 \mathrm{~mol} \%)$ in CPME $(0.2 \mathrm{~mL})$, then 2-hydroxycinnamaldehyde 2a ( $0.12 \mathrm{mmol}, 17.8 \mathrm{mg}, 1.2$ equiv.), 3-chlorobenzoic acid (0.02 mmol, $3.1 \mathrm{mg}, 20 \mathrm{~mol} \%)$ and the aldehyde 7a (0.1 mmol, $17.2 \mathrm{mg}, 1.0$ equiv.) was added in one portion and the resulting solution was stirred for about 13 hours at $40{ }^{\circ} \mathrm{C}$ in oil bath until the material 1 disappeared. After completion of the reaction, the crude product was directly put into the next step without column chromatography separation. The crude product (1.0 equiv.) was respectively dissolved in $\mathrm{CH}_{2} \mathrm{Cl}_{2}(0.2 \mathrm{mmol}$ in $1 \mathrm{~mL})$ at $0{ }^{\circ} \mathrm{C}$ in ice bath. TEA (6.0 equiv.), $\mathrm{MsCl}$ (2.0 equiv.), DMAP (0.2 equiv.) were added to the reaction mixtures. After full conversion of the reaction about 8 hours, the reaction mixture was purified by flash chromatography on silica gel (petroleum ether/ethyl acetate $=$ $60 / 1$ ) to give product 8 as a white solid $17 \mathrm{mg}$ in $56 \%$ yield for two steps. ${ }^{1} \mathbf{H}$ NMR $\left(400 \mathrm{MHz}, \mathrm{CDCl}_{3}\right) \delta 7.49-7.42(\mathrm{~m}, 2 \mathrm{H}), 7.41-7.30(\mathrm{~m}, 4 \mathrm{H}), 7.33-7.25(\mathrm{~m}, 4 \mathrm{H}), 7.20(\mathrm{t}, J$ $=7.3 \mathrm{~Hz}, 1 \mathrm{H}), 7.05-6.97(\mathrm{~m}, 2 \mathrm{H}), 6.22(\mathrm{dd}, J=6.2,2.5 \mathrm{~Hz}, 1 \mathrm{H}), 6.08(\mathrm{dd}, J=5.4,2.4 \mathrm{~Hz}$, $1 \mathrm{H}), 5.70(\mathrm{~d}, J=2.1 \mathrm{~Hz}, 1 \mathrm{H}), 4.78-4.71(\mathrm{~m}, 1 \mathrm{H}), 3.41(\mathrm{tq}, J=4.9,2.3 \mathrm{~Hz}, 1 \mathrm{H}), 3.27-3.14$ (m, 2H), 2.48 (tdd, $J=13.2,5.1,2.5 \mathrm{~Hz}, 1 \mathrm{H}$ ), 2.23 (ddt, $J=12.5,6.3,1.8 \mathrm{~Hz}, 1 \mathrm{H}$ ) ppm. ${ }^{13} \mathrm{C}$ NMR $\left(100 \mathrm{MHz}, \mathrm{CDCl}_{3}\right) \delta 151.0,141.7,138.7,136.8,128.5,127.8,127.4,126.3,125.5$, 125.0, 124.7, 121.8, 117.0, 103.2, 95.1, 34.8, 33.6, 32.0, 25.5 ppm. HRMS: $[\mathrm{M}+\mathrm{H}]^{+}$calcd. For $\mathrm{C}_{21} \mathrm{H}_{20} \mathrm{O}_{2}{ }^{+}$303.1380, found 303.1387. $[\alpha]_{\mathbf{D}}{ }^{20}+46.68\left(c=1.42\right.$ in $\left.\mathrm{CHCl}_{3}\right)$. The enantiomeric excess was determined by HPLC analysis on Daicel Chiralpak IB column 
$[n$-hexane $/ i-\operatorname{PrOH}=90 / 10,1 \mathrm{~mL} / \mathrm{min}], \lambda=205 \mathrm{~nm}, t_{\text {major }}=6.45 \mathrm{~min}, t_{\text {minor }}=8.20 \mathrm{~min}$, ee $>\mathbf{9 9 \%}$. The diastereomeric ratio was determined by ${ }^{1} \mathrm{H} N M R, \boldsymbol{d r}>\mathbf{2 0 : 1}$.

\section{E2. Synthesis of 9}

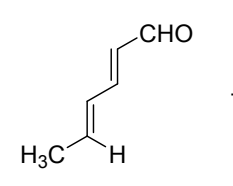

7b<smiles>O=C/C=C/c1ccccc1O</smiles>

$2 a$

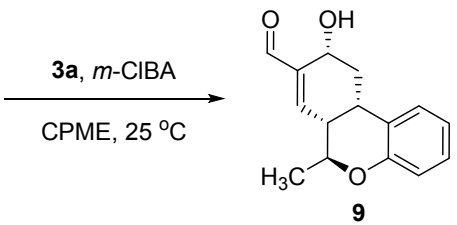

9

Detailed method: A glass vial equipped with a magnetic stirring bar was charged with catalyst 3a (0.02 mmol, $20 \mathrm{~mol} \%$.) in CPME $(0.2 \mathrm{~mL})$, then 2-hydroxycinnamaldehyde (0.12 mmol, $17.8 \mathrm{mg}, 1.2$ equiv.), $m$-ClBA (0.02 mmol, $2.4 \mathrm{mg}, 20 \mathrm{~mol} \%$ ) and the $(E, E)$-2,4-hexadienal $\mathbf{7 b}(0.1 \mathrm{mmol}, 9.6 \mathrm{mg}, 1.0$ equiv.) were added in one portion and the resulting solution was stirred for about 8 hours at $25{ }^{\circ} \mathrm{C}$ until the material $\mathbf{7 b}$ disappeared. The reaction mixture was purified by flash chromatography on silica gel (petroleum ether/ethyl acetate $=6 / 1$ ) to give product 9 as a yellow solid $7 \mathrm{mg}$ in $28 \%$ yield. ${ }^{1} \mathrm{H}$ NMR $\left(400 \mathrm{MHz}, \mathrm{CDCl}_{3}\right) \delta 9.52(\mathrm{~s}, 1 \mathrm{H}), 7.21-7.12(\mathrm{~m}, 2 \mathrm{H}), 6.94(\mathrm{td}, J=7.5,1.3$ $\mathrm{Hz}, 1 \mathrm{H}), 6.87$ (dd, $J=8.1,1.3 \mathrm{~Hz}, 1 \mathrm{H}), 6.84(\mathrm{dd}, J=5.1,1.2 \mathrm{~Hz}, 1 \mathrm{H}), 4.85-4.76(\mathrm{~m}, 1 \mathrm{H})$, $4.05(\mathrm{dq}, J=9.2,6.2 \mathrm{~Hz}, 1 \mathrm{H}), 3.88(\mathrm{~s}, 1 \mathrm{H}), 2.95$ (ddd, $J=13.6,5.4,3.3 \mathrm{~Hz}, 1 \mathrm{H}), 2.66$ (tdd, $J$ $=9.2,4.0,2.6 \mathrm{~Hz}, 1 \mathrm{H}), 2.50-2.40(\mathrm{~m}, 1 \mathrm{H}), 1.76(\mathrm{td}, J=13.5,10.1 \mathrm{~Hz}, 1 \mathrm{H}), 1.55(\mathrm{~d}, J=6.1$ $\mathrm{Hz}, 3 \mathrm{H}) \mathrm{ppm} .{ }^{13} \mathrm{C}$ NMR $\left(100 \mathrm{MHz}, \mathrm{CDCl}_{3}\right) \delta 195.3,154.3,149.89,144.6,129.5,127.9$, 124.6, 121.1, 116.9, 71.0, 65.3, 41.4, 36.8, 32.2, 19.3 ppm. HRMS: [M+Na] calcd. For $\mathrm{C}_{15} \mathrm{H}_{16} \mathrm{O}_{3} \mathrm{Na}^{+}$267.0992, found 267.0994. $[\alpha]_{\mathbf{D}}{ }^{20}-16.22\left(c=0.47\right.$ in $\left.\mathrm{CHCl}_{3}\right)$. The enantiomeric excess was determined by HPLC analysis on Daicel Chiralpak IC column $[n$-hexane $/ i-\mathrm{PrOH}=90 / 10,1 \mathrm{~mL} / \mathrm{min}], \lambda=220 \mathrm{~nm}, t_{\text {major }}=39.79 \mathrm{~min}, t_{\text {minor }}=21.79 \mathrm{~min}$, ee $=86 \%$. 


\section{F. Synthetic transformation}

The first way of transformation:

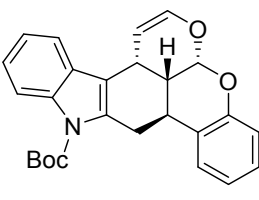

$6 a$

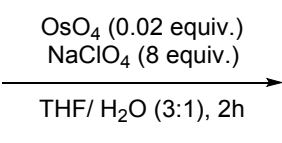

THF/ $\mathrm{H}_{2} \mathrm{O}(3: 1), 2 \mathrm{~h}$

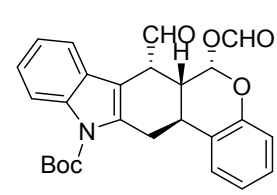

10

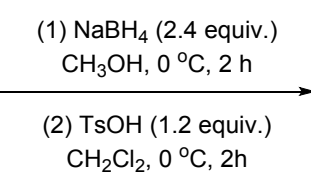

$\mathrm{CH}_{2} \mathrm{Cl}_{2}, 0{ }^{\circ} \mathrm{C}, 2 \mathrm{~h}$

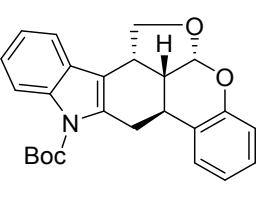

11

A glass vial equipped with a magnetic stirring bar containing $6 \mathbf{6}(21 \mathrm{mg}, 0.05 \mathrm{mmol})$ in a mixture of THF and $\mathrm{H}_{2} \mathrm{O}(3: 1,0.8 \mathrm{~mL})$ solution was added $\mathrm{OsO}_{4}(50 \mu \mathrm{L}, 0.001 \mathrm{mmol})$. Then $\mathrm{NaIO}_{4}(86 \mathrm{mg}, 0.4 \mathrm{mmol})$ was added to the reaction solution. After stirring at room temperature for about 2 hours (TLC monitoring) the mixture was diluted with saturated $\mathrm{NaS}_{2} \mathrm{O}_{3}$ solution and extracted with $\mathrm{CH}_{2} \mathrm{Cl}_{2}$ and $\mathrm{H}_{2} \mathrm{O}$ until no product was visible in TLC. The organic layer was dried with $\mathrm{Na}_{2} \mathrm{SO}_{4}$, filtered and the filtrate was evaporated and obtained crude was purified by column chromatography on a silica gel (petroleum ether/ethyl acetate $=10 / 1)$ to get product 10 as white solid $(12 \mathrm{mg}, 54 \%)$.

The solution of 10 (31 mg, $0.07 \mathrm{mmol}$ ) in $\mathrm{MeOH}(0.5 \mathrm{~mL})$ was added $\mathrm{NaBH}_{4}(6.4 \mathrm{mg}$, $0.16 \mathrm{mmol}$ ) at $0{ }^{\circ} \mathrm{C}$. After the reaction completed, the mixture was diluted with saturated $\mathrm{NH}_{4} \mathrm{Cl}$ solution and extracted with ethyl acetate and $\mathrm{H}_{2} \mathrm{O}$. The organic layer was dried with $\mathrm{Na}_{2} \mathrm{SO}_{4}$, filtered and the filtrate was evaporated and purified by column chromatograph on a silica gel (petroleum ether/ethyl acetate $=3 / 1$ ) to get compound alcohol intermediate as white solid. To a solution of alcohol intermediate in $\mathrm{CH}_{2} \mathrm{Cl}_{2}(0.5$ $\mathrm{mL}$ ) was added TsOH (14.5 mg, $0.08 \mathrm{mmol}$ ). After the reaction completed (detected by TLC), the mixture was diluted with saturated $\mathrm{NaHCO}_{3}$ solution and extracted with $\mathrm{CH}_{2} \mathrm{Cl}_{2}$ and $\mathrm{H}_{2} \mathrm{O}$. The organic layer was dried with $\mathrm{Na}_{2} \mathrm{SO}_{4}$, filtered and the filtrate was evaporated and purified by column chromatograph on a silica gel (petroleum ether/ethyl acetate $=14 / 1)$ to get compound 11 as white solid (14 mg, 50\%). 


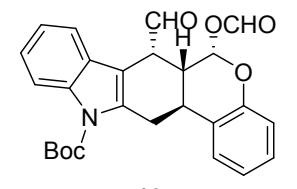

10

tert-butyl(6S,6aR,7R,13aS)-7-formyl-6-(formyloxy)-6a,7,13,13a-t

etrahydrochromeno[4,3-b] carbazole-12(6H)-carboxylate

(10) : ${ }^{1} \mathrm{H}$ NMR (400 MHz, $\left.\mathrm{CDCl}_{3}\right) \delta 9.65(\mathrm{~d}, J=5.5 \mathrm{~Hz}, 1 \mathrm{H}), 8.14(\mathrm{~d}, J=$ $8.3 \mathrm{~Hz}, 1 \mathrm{H}), 8.03(\mathrm{~s}, 1 \mathrm{H}), 7.44(\mathrm{~d}, J=7.7 \mathrm{~Hz}, 1 \mathrm{H}), 7.37(\mathrm{~d}, J=7.6 \mathrm{~Hz}, 1 \mathrm{H}), 7.31(\mathrm{t}, J=7.3 \mathrm{~Hz}$, 1H), $7.22(\mathrm{~d}, J=7.8 \mathrm{~Hz}, 2 \mathrm{H}), 7.10(\mathrm{t}, J=7.2 \mathrm{~Hz}, 1 \mathrm{H}), 6.95(\mathrm{~d}, J=8.1 \mathrm{~Hz}, 1 \mathrm{H}), 6.76(\mathrm{~d}, J=1.3$ $\mathrm{Hz}, 1 \mathrm{H}), 4.26(\mathrm{dd}, J=18.0,6.0 \mathrm{~Hz}, 1 \mathrm{H}), 3.92(\mathrm{t}, J=5.3 \mathrm{~Hz}, 1 \mathrm{H}), 3.90-3.82(\mathrm{~m}, 1 \mathrm{H}), 3.03$ (dd, $J=17.7,10.7 \mathrm{~Hz}, 1 \mathrm{H}), 2.62(\mathrm{dd}, J=13.1,3.2 \mathrm{~Hz}, 1 \mathrm{H}), 1.74(\mathrm{~s}, 9 \mathrm{H}) \mathrm{ppm} .{ }^{13} \mathbf{C}$ NMR $(100$ $\left.\mathrm{MHz}, \mathrm{CDCl}_{3}\right) \delta 197.6,158.9,150.3,149.8,136.5,136.1,128.5,127.8,126.7,124.6,124.3$, 123.3, 122.7, 117.9, 117.8, 115.8, 109.5, 90.3, 84.5, 47.5, 39.8, 31.6, 28.3, 27.7 ppm. HRMS: $[\mathrm{M}+\mathrm{H}]^{+}$calcd. For $\mathrm{C}_{26} \mathrm{H}_{26} \mathrm{NO}_{6}{ }^{+} 448.1755$, found 448.1751. $[\alpha]_{\mathbf{D}}{ }^{20}+192.43(c=1$ in $\mathrm{CHCl}_{3}$ ). The enantiomeric excess was determined by HPLC analysis on Daicel Chiralpak IB column $[n$-hexane $/ i-\mathrm{PrOH}=90 / 10,1 \mathrm{~mL} / \mathrm{min}], \lambda=205 \mathrm{~nm}, t_{\text {major }}=37.59 \mathrm{~min}, t_{\text {minor }}=$ $50.40 \mathrm{~min}$, ee $>\mathbf{9 9 \%}$. The diastereomeric ratio was determined by ${ }^{1} \mathrm{H} \mathrm{NMR}, \boldsymbol{d r}>\mathbf{2 0 : 1}$.

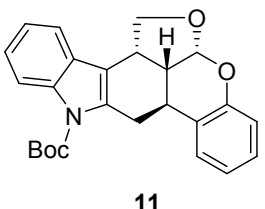

tert-butyl(2aR,2a1R,7bS,13cR)-1,2a,2a1,7b,8,13c-hexahydro-9Hbenzo[3,4]furo[4',3',2':8,1]isochromeno[6,7-b]indole-9-carboxyl ate (11) : ${ }^{1} \mathrm{H}$ NMR $\left(500 \mathrm{MHz}, \mathrm{CDCl}_{3}\right) \delta 8.17(\mathrm{~d}, J=8.3 \mathrm{~Hz}, 1 \mathrm{H}), 7.41(\mathrm{~d}, J$ $=6.8 \mathrm{~Hz}, 1 \mathrm{H}), 7.34-7.28(\mathrm{~m}, 3 \mathrm{H}), 7.26(\mathrm{dd}, J=7.3,1.2 \mathrm{~Hz}, 1 \mathrm{H}), 7.12-7.06(\mathrm{~m}, 2 \mathrm{H}), 5.92$ $(\mathrm{d}, J=5.0 \mathrm{~Hz}, 1 \mathrm{H}), 4.64(\mathrm{t}, J=8.1 \mathrm{~Hz}, 1 \mathrm{H}), 4.08(\mathrm{dd}, J=10.5,8.3 \mathrm{~Hz}, 1 \mathrm{H}), 3.97-3.89(\mathrm{~m}$, 1H), $3.83-3.77(\mathrm{~m}, 1 \mathrm{H}), 3.16-3.04(\mathrm{~m}, 2 \mathrm{H}), 2.41$ (ddd, J=11.9, 6.6, $4.9 \mathrm{~Hz}, 1 \mathrm{H}), 1.77$ (s, 9H) ppm. ${ }^{13} \mathrm{C}$ NMR $\left(125 \mathrm{MHz}, \mathrm{CDCl}_{3}\right) \delta 153.0,150.4,136.1,135.5,129.4,129.0,127.8$, 124.1, 123.6, 122.9, 122.3, 117.7, 117.5, 115.8, 114.8, 104.5, 84.1, 71.4, 45.1, 37.1, 29.8, 28.4, 27.2 ppm. HRMS: $[\mathrm{M}+\mathrm{H}]^{+}$calcd. For $\mathrm{C}_{25} \mathrm{H}_{26} \mathrm{NO}_{4}{ }^{+}$404.1817, found 404.1813. $[\alpha]_{\mathbf{D}}{ }^{20}$ -83.78 $\left(c=1.25\right.$ in $\left.\mathrm{CHCl}_{3}\right)$. The enantiomeric excess was determined by HPLC analysis on Daicel Chiralpak OD-H column $[n$-hexane $/ i$-PrOH $=80 / 20,1 \mathrm{~mL} / \mathrm{min}], \lambda=225 \mathrm{~nm}, t_{\text {major }}$ $=6.13 \mathrm{~min}, t_{\text {minor }}=10.36 \mathrm{~min}, \mathbf{e e}>\mathbf{9 9 \%}$. The diastereomeric ratio was determined by ${ }^{1} \mathrm{H}$ NMR, $d r>20: 1$. 


\section{The second way of transformation:}

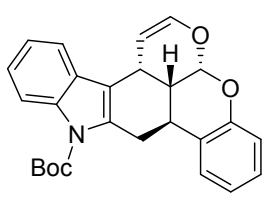

$6 a$

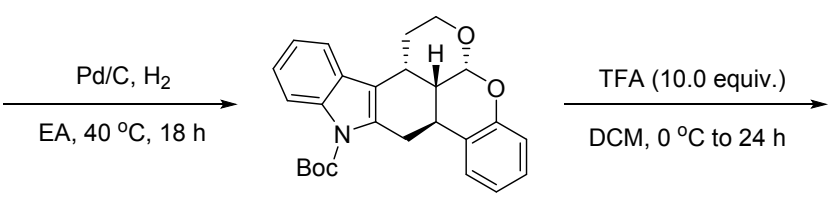

12

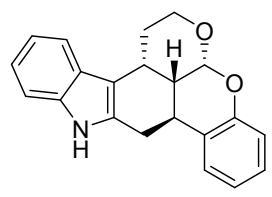

13

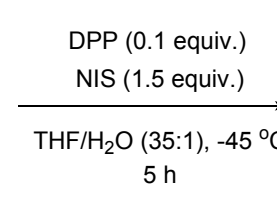

$5 \mathrm{~h}$

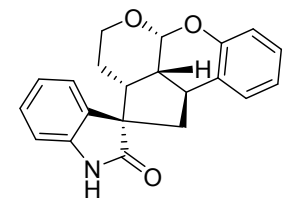

14

Hydrogenate a solution of $\mathbf{6 a}(49 \mathrm{mg}, 0.12 \mathrm{mmol})$ in ethyl acetate $(1 \mathrm{~mL})$ at atmospheric pressure using $20 \% \mathrm{Pd} / \mathrm{C}(9.8 \mathrm{mg})$ as the catalyst. And the reaction mixture was stirred at $40^{\circ} \mathrm{C}$ in oil bath. After completion of the reaction (18 hours), filter the catalyst and the solvent was removed under vacuum. The product was purified by column chromatography on a silica gel (petroleum ether/ethyl acetate $=20 / 1$ ) to afford the desired product $\mathbf{1 1}$ as a white solid (20 mg, 40\%).

To a solvent of 12 in $\mathrm{CH}_{2} \mathrm{Cl}_{2}(0.5 \mathrm{~mL})$ was added trifluoroacetic acid (30 $\left.\mu \mathrm{l}, 0.4 \mathrm{mmol}\right)$ at $0{ }^{\circ} \mathrm{C}$ in ice bath. Then the reaction mixture was stirred at room temperature for 24 hours. After the reaction completed (detected by TLC), the mixture was diluted with saturated $\mathrm{NaHCO}_{3}$ solution and extracted with $\mathrm{CH}_{2} \mathrm{Cl}_{2}$ and $\mathrm{H}_{2} \mathrm{O}$. The organic layer was dried with $\mathrm{Na}_{2} \mathrm{SO}_{4}$, filtered and the filtrate was evaporated and purified by column chromatograph on a silica gel (petroleum ether/ethyl acetate $=10 / 1$ ) to get compound 13 as white solid (6 mg, 45\%).

At room temperature, to a vial charged with diphenyl phosphate $(1.3 \mathrm{mg}, 10 \mathrm{~mol} \%)$ and the indole $13(15 \mathrm{mg}, 0.047 \mathrm{mmol})$ was added the mixed solvent of THF/ $\mathrm{H}_{2} \mathrm{O}(432 \mu \mathrm{L}$, $\mathrm{v} / \mathrm{v}=35: 1$ ). The mixture was cooled to $-45^{\circ} \mathrm{C}$ and followed by slow addition of NIS (16 $\mathrm{mg}, 0.071 \mathrm{mmol}$ ). The mixture was stirred at $-45{ }^{\circ} \mathrm{C}$, and the reaction progress was monitored by TLC. Upon completion 5 hours, the reaction was quenched by addition of a saturated aqueous $\mathrm{Na}_{2} \mathrm{SO}_{3}$ solution $(3 \mathrm{~mL})$. Next, the mixture was extracted with ethyl acetate and $\mathrm{H}_{2} \mathrm{O}$, dried over anhydrous $\mathrm{Na}_{2} \mathrm{SO}_{4}$, filtered, and concentrated. The residue 
was subjected to silica gel column chromatography (petroleum ether/ethyl acetate $=$ 8/1) to afford the pure product 14 as white solid (15 mg, 96\%).

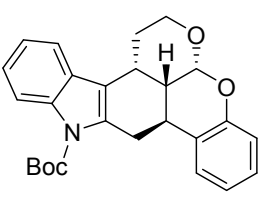

12

tert-butyl(3aR,3a1S,8bS,14cR)-1,3a,3a1,8b,9,14c-hexahydrochro meno $\left[4^{\prime}, 3^{\prime}, 2 ': 8,1\right]$ isochromeno $[6,5-b]$ indole-10(2H)-carboxylate (12): ${ }^{1} \mathrm{H}$ NMR (400 MHz, $\left.\mathrm{CDCl}_{3}\right) \delta 8.19(\mathrm{~d}, J=8.1 \mathrm{~Hz}, 1 \mathrm{H}), 7.48-7.42$ (m, 1H), 7.36 (d, $J=7.7 \mathrm{~Hz}, 1 \mathrm{H}), 7.30$ (dd, $J=7.3,1.5 \mathrm{~Hz}, 1 \mathrm{H}), 7.26-$ $7.22(\mathrm{~m}, 1 \mathrm{H}), 7.19(\mathrm{t}, J=7.7 \mathrm{~Hz}, 1 \mathrm{H}), 7.01(\mathrm{td}, J=7.4,1.3 \mathrm{~Hz}, 1 \mathrm{H}), 6.96(\mathrm{dd}, J=8.1,1.3 \mathrm{~Hz}$, $1 \mathrm{H}), 5.38(\mathrm{~d}, J=2.5 \mathrm{~Hz}, 1 \mathrm{H}), 4.17(\mathrm{dd}, J=11.8,4.9 \mathrm{~Hz}, 1 \mathrm{H}), 4.03(\mathrm{dd}, J=17.6,5.8 \mathrm{~Hz}, 1 \mathrm{H}$ ), $3.85(\mathrm{td}, J=12.4,2.5 \mathrm{~Hz}, 1 \mathrm{H}), 3.53(\mathrm{td}, J=11.8,5.8 \mathrm{~Hz}, 1 \mathrm{H}), 3.33(\mathrm{dt}, J=10.9,4.9 \mathrm{~Hz}, 1 \mathrm{H})$, 2.92 (dd, $J=17.6,11.2 \mathrm{~Hz}, 1 \mathrm{H}$ ), 2.19 (ddd, $J=12.5,4.5,2.5 \mathrm{~Hz}, 1 \mathrm{H}), 2.12-2.04(\mathrm{~m}, 1 \mathrm{H}$ ), $1.83(\mathrm{td}, J=13.1,5.0 \mathrm{~Hz}, 1 \mathrm{H}), 1.73(\mathrm{~s}, 9 \mathrm{H}) \mathrm{ppm} .{ }^{13} \mathbf{C} \mathbf{N M R}\left(100 \mathrm{MHz}, \mathrm{CDCl}_{3}\right) \delta 151.1,150.5$, $136.3,134.2,128.2,127.9,126.3,124.0,123.9,122.7,121.6,119.6,117.5,116.8,115.9$, 97.6, 83.8, 66.2, 36.3, 31.3, 31.0, 28.6, 28.4, 25.6 ppm. HRMS: $[\mathrm{M}+\mathrm{H}]^{+}$calcd. For $\mathrm{C}_{26} \mathrm{H}_{28} \mathrm{NO}_{4}{ }^{+}$418.2013, found 418.2018. $[\alpha]_{\mathrm{D}}{ }^{20}+106.70\left(c=2.08\right.$ in $\left.\mathrm{CHCl}_{3}\right)$. The enantiomeric excess was determined by HPLC analysis on Daicel Chiralpak IB column $[n$-hexane $/ i-\operatorname{PrOH}=90 / 10,1 \mathrm{~mL} / \mathrm{min}], \lambda=225 \mathrm{~nm}, t_{\text {major }}=7.97 \mathrm{~min}$, ee $>\mathbf{9 9 \%}$. The diastereomeric ratio was determined by ${ }^{1} \mathrm{H}$ NMR, $\boldsymbol{d r} \mathbf{> 2 0 : 1}$.

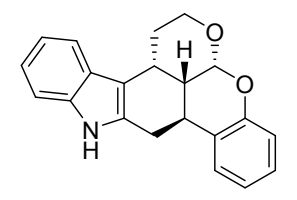

13

(3aR,3a1S,8bS,14cR)-1,2,3a,3a1,8b,9,10,14c-octahydrochromeno $\left[4^{\prime}, 3^{\prime}, 2^{\prime}: 8,1\right]$ isochromeno[6,5-b] indole (13): ${ }^{1} \mathrm{H}$ NMR $(400 \mathrm{MHz}$, $\left.\mathrm{CDCl}_{3}\right) \delta 7.86(\mathrm{~s}, 1 \mathrm{H}), 7.51(\mathrm{~d}, J=7.6 \mathrm{~Hz}, 1 \mathrm{H}), 7.34(\mathrm{~d}, J=8.0 \mathrm{~Hz}, 1 \mathrm{H})$, $7.28(\mathrm{~d}, J=7.7 \mathrm{~Hz}, 1 \mathrm{H}), 7.22-7.15(\mathrm{~m}, 2 \mathrm{H}), 7.12(\mathrm{t}, J=7.4 \mathrm{~Hz}, 1 \mathrm{H})$, $6.99(\mathrm{dd}, J=16.6,7.8 \mathrm{~Hz}, 2 \mathrm{H}), 5.38(\mathrm{~d}, J=2.5 \mathrm{~Hz}, 1 \mathrm{H}), 4.16(\mathrm{dd}, J=11.8,5.0 \mathrm{~Hz}, 1 \mathrm{H}), 3.85$ (ddd, $J=13.0,11.8,2.5 \mathrm{~Hz}, 1 \mathrm{H}$ ), $3.66-3.52(\mathrm{~m}, 2 \mathrm{H}), 3.39$ (dt, $J=12.1,4.8 \mathrm{~Hz}, 1 \mathrm{H}$ ), 2.80 (dd, $J=15.2,10.7 \mathrm{~Hz}, 1 \mathrm{H}$ ), 2.26 (ddd, $J=12.1,4.5,2.5 \mathrm{~Hz}, 1 \mathrm{H}$ ), 2.09 (dd, $J=13.8,4.3 \mathrm{~Hz}$, 1H), 1.81 (qd, $J=12.9,5.1 \mathrm{~Hz}, 1 \mathrm{H}) \mathrm{ppm} .{ }^{13} \mathbf{C}$ NMR $\left(100 \mathrm{MHz}, \mathrm{CDCl}_{3}\right) \delta 151.3,136.1,132.3$, $128.0,126.5,126.2,123.9,121.6,121.5,119.6,117.7,116.9,113.8,110.9,98.0,66.4,37.5$ 31.5, 29.5, 28.4, 25.8 ppm. HRMS: $[\mathrm{M}+\mathrm{H}]^{+}$calcd. For $\mathrm{C}_{21} \mathrm{H}_{20} \mathrm{NO}_{2}{ }^{+} 318.1449$, found 318.1442. $[\alpha]_{\mathbf{D}}{ }^{20}+86.11\left(c=0.83\right.$ in $\left.\mathrm{CHCl}_{3}\right)$. The enantiomeric excess was determined by 
HPLC analysis on Daicel Chiralpak IC column [ $n$-hexane $/ i-\mathrm{PrOH}=90 / 10,1 \mathrm{~mL} / \mathrm{min}], \lambda=$ $225 \mathrm{~nm}, t_{\text {major }}=23.45 \mathrm{~min}$, ee $>\mathbf{9 9 \%}$. The diastereomeric ratio was determined by ${ }^{1} \mathrm{H}$ NMR, $\boldsymbol{d} \boldsymbol{r}>\mathbf{2 0 : 1}$.

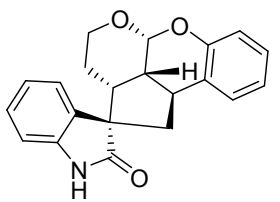

14

(2S,2aR,2a1R,5aR,10bS)-1,2a,2a1,3,5a,10b-hexahydro-4H-5,6-di oxaspiro[aceanthrylene-2,3'-indolin]-6a(10a),7,9-trien-2'-one (14): ${ }^{1}$ H NMR (400 MHz, DMSO- $\left.d_{6}\right) \delta 10.84(\mathrm{~s}, 1 \mathrm{H}), 7.45(\mathrm{~d}, J=7.7 \mathrm{~Hz}$, $1 \mathrm{H}), 7.37(\mathrm{~d}, J=7.8 \mathrm{~Hz}, 1 \mathrm{H}), 7.29(\mathrm{~d}, J=7.8 \mathrm{~Hz}, 1 \mathrm{H}), 7.17(\mathrm{t}, J=7.6 \mathrm{~Hz}$, 1H), $7.04-6.93(\mathrm{~m}, 3 \mathrm{H}), 6.84(\mathrm{dd}, J=8.1,1.3 \mathrm{~Hz}, 1 \mathrm{H}), 5.35(\mathrm{~d}, J=2.4 \mathrm{~Hz}, 1 \mathrm{H}), 3.98(\mathrm{dd}, J$ $=11.6,4.8 \mathrm{~Hz}, 1 \mathrm{H}), 3.76(\mathrm{td}, J=12.2,2.3 \mathrm{~Hz}, 1 \mathrm{H}), 3.60(\mathrm{dd}, J=15.8,5.6 \mathrm{~Hz}, 1 \mathrm{H}), 3.40(\mathrm{ddd}$ $J=21.4,10.7,5.2 \mathrm{~Hz}, 4 \mathrm{H}), 2.65(\mathrm{dd}, J=15.8,11.2 \mathrm{~Hz}, 1 \mathrm{H}), 2.16(\mathrm{ddd}, J=12.3,4.5,2.5 \mathrm{~Hz}$, 1H), 1.99 (dd, $J=13.7,4.8 \mathrm{~Hz}, 1 \mathrm{H}), 1.60(\mathrm{qd}, J=12.8,5.0 \mathrm{~Hz}, 1 \mathrm{H}) \mathrm{ppm} .{ }^{13} \mathbf{C}$ NMR ${ }^{13} \mathrm{C}$ NMR $\left(100 \mathrm{MHz}, \mathrm{DMSO}-d_{6}\right) \delta 151.6,136.5,133.4,128.1,127.2,126.6,124.8,121.6,120.8$, $118.8,117.8,116.5,112.7,111.3,97.7,65.9,37.4,31.2,29.7,28.5,25.7$. HRMS: $[M+H]^{+}$ calcd. For $\mathrm{C}_{21} \mathrm{H}_{20} \mathrm{NO}_{3}{ }^{+} 334.1398$, found 334.1395. $[\alpha]_{\mathbf{D}}{ }^{20}+55.3\left(c=0.83\right.$ in $\left.\mathrm{CHCl}_{3}\right)$. The enantiomeric excess was determined by HPLC analysis on Daicel Chiralpak OD-H column $[n$-hexane $/ i-\mathrm{PrOH}=80 / 20,1 \mathrm{~mL} / \mathrm{min}], \lambda=225 \mathrm{~nm}, t_{\text {major }}=23.28 \mathrm{~min}, t_{\text {minor }}=$ 22.06 min, ee $>\mathbf{9 9} \%$. The diastereomeric ratio was determined by ${ }^{1} \mathrm{H}$ NMR, $\boldsymbol{d r}>\mathbf{2 0 : 1}$.

\section{The third way of transformation:}

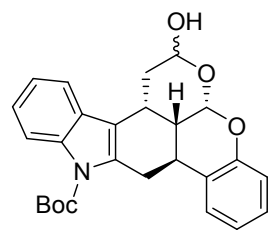

$5 a$

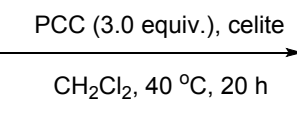

$\mathrm{CH}_{2} \mathrm{Cl}_{2}, 40^{\circ} \mathrm{C}, 20 \mathrm{~h}$

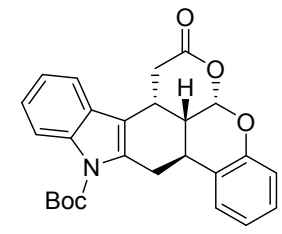

15

To a solvent of $5 \mathbf{a}(22 \mathrm{mg}, 0.5 \mathrm{mmol})$ in $\mathrm{CH}_{2} \mathrm{Cl}_{2}(0.5 \mathrm{~mL})$ was added celite $(32 \mathrm{mg})$ and PCC (32 mg, $0.4 \mathrm{mmol})$ at $40{ }^{\circ} \mathrm{C}$ in oil bath. Then the reaction mixture was stirred for 20 hours. After the reaction completed (detected by TLC), the mixture was purified by column chromatograph on a silica gel (petroleum ether/ethyl acetate $=8 / 1$ ) to get compound 15 as white solid (6 mg, 28\%). 
tert-butyl(3aS,3a1S,8bS,14cR)-2-oxo-1,3a,3a1,8b,9,14c-hexahydrochromeno[4',3', 2':8,1]isochromeno[6,5-b]indole-10(2H)-carboxylate (15): ${ }^{1} \mathrm{H}$ NMR (400 MHz, $\left.\mathrm{CDCl}_{3}\right) \delta 8.17(\mathrm{~d}, J=8.4 \mathrm{~Hz}, 1 \mathrm{H}), 7.41-7.35(\mathrm{~m}, 2 \mathrm{H}), 7.35-7.26(\mathrm{~m}, 3 \mathrm{H}), 7.08(\mathrm{td}, J=7.5$, $1.3 \mathrm{~Hz}, 1 \mathrm{H}$ ), 7.02 (dd, $J=8.1,1.3 \mathrm{~Hz}, 1 \mathrm{H}), 6.16(\mathrm{~d}, J=2.3 \mathrm{~Hz}, 1 \mathrm{H}$ ), 4.10 (dd, $J=17.5,5.4 \mathrm{~Hz}$, 1H), $3.73-3.65(\mathrm{~m}, 1 \mathrm{H}), 3.24(\mathrm{dd}, J=18.6,10.2 \mathrm{~Hz}, 1 \mathrm{H}), 2.94$ (ddd, $J=17.6,11.0,1.7 \mathrm{~Hz}$, 1H), $2.72(\mathrm{dd}, J=18.6,6.8 \mathrm{~Hz}, 1 \mathrm{H}), 2.53(\mathrm{ddd}, J=12.8,5.5,2.3 \mathrm{~Hz}, 1 \mathrm{H}), 1.73(\mathrm{~s}, 9 \mathrm{H}) \mathrm{ppm}$. ${ }^{13}$ C NMR $\left(100 \mathrm{MHz}, \mathrm{CDCl}_{3}\right) \delta 168.8,150.4,150.2,136.2,134.0,128.6,127.6,126.3,124.4$, $123.1,122.7,118.0,117.4,117.3,116.0,97.0,84.3,34.7,33.4,30.0,29.7,28.3,26.0,25.7$ ppm. HRMS: $[\mathrm{M}+\mathrm{H}]^{+}$calcd. For $\mathrm{C}_{26} \mathrm{H}_{26} \mathrm{O}_{5} \mathrm{~N}^{+}$432.1811, found 432.1805. [ $\left.\alpha\right]_{\mathbf{D}}{ }^{20}+69.84(c=$ 0.33 in $\mathrm{CHCl}_{3}$ ). The enantiomeric excess was determined by HPLC analysis on Daicel Chiralpak IA column $[n$-hexane $/ i-\mathrm{PrOH}=70 / 30,1 \mathrm{~mL} / \mathrm{min}], \lambda=205 \mathrm{~nm}, t_{\text {major }}=7.84 \mathrm{~min}$, $t_{\text {minor }}=11.75 \mathrm{~min}, \mathbf{e e}=\mathbf{9 9 \%}$. The diastereomeric ratio was determined by ${ }^{1} \mathrm{H} \mathrm{NMR}$, $d r>20: 1$.

The fourth way of transformation:
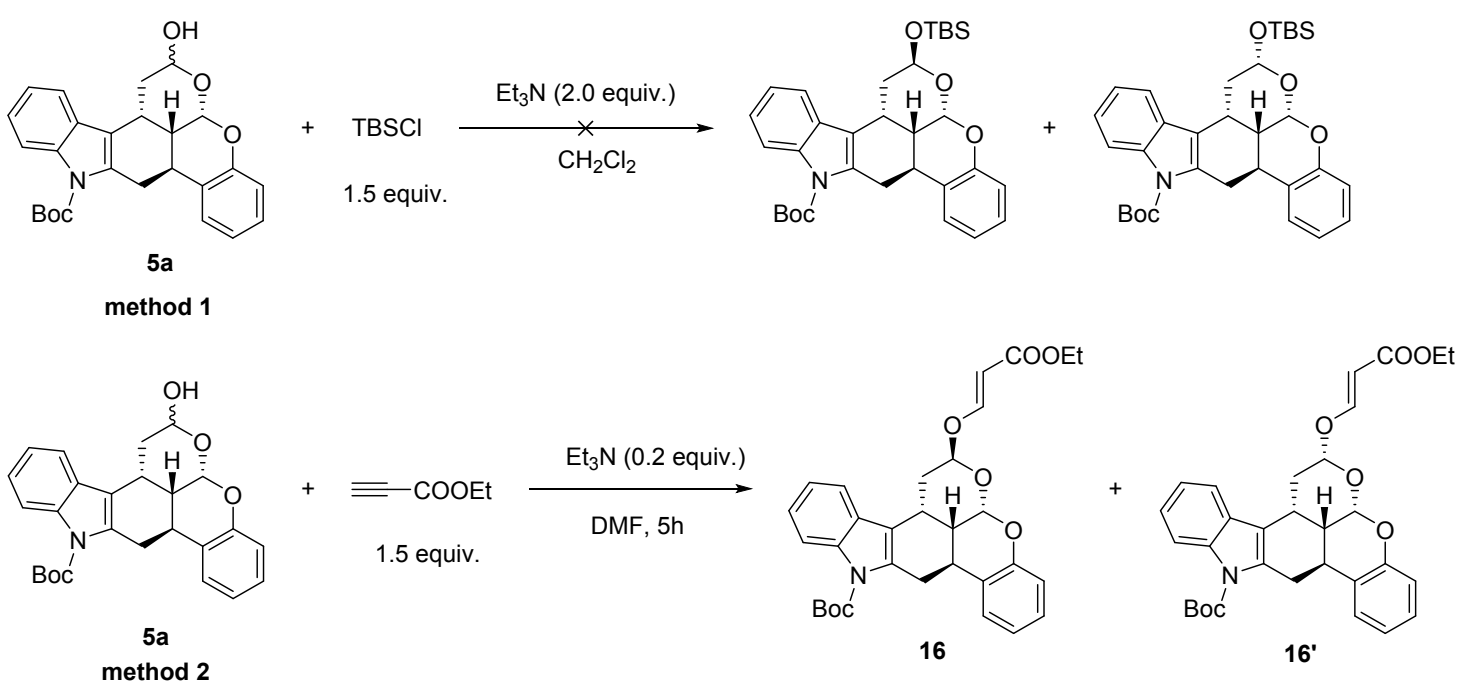

Attempts to separate the epimeric mixture of 5a upon derivatization, such as silylation or acylation (method 1), failed to afford any of the desired products.

Method 2: A glass vial equipped with a magnetic stirring bar containing 5 a $(22 \mathrm{mg}$, $0.05 \mathrm{mmol}$ ) in a dimethyl formamide (DMF, $0.2 \mathrm{~mL}$ ) solution was added TEA ( $2 \mu \mathrm{L}, 0.01$ mmol). Then ethyl propiolate ( $8 \mu \mathrm{L}, 0.075 \mathrm{mmol}$ ) was added to the reaction solution. After stirring at room temperature for about 5 hours (TLC monitoring) the mixture was 
extracted with ethyl acetate and $\mathrm{H}_{2} \mathrm{O}$ until no product was visible in TLC. The organic layer was dried with $\mathrm{Na}_{2} \mathrm{SO}_{4}$, filtered and the filtrate was evaporated and obtained crude was purified by column chromatography on a silica gel (petroleum ether/ethyl acetate $=$ 50/1) to get product 16 as white solid (10 mg, 39\%) and the same silica gel (petroleum ether/ethyl acetate $=20 / 1)$ to get product $\mathbf{1 6}^{\prime}$ as white solid (11 $\left.\mathrm{mg}, 41 \%\right)$.

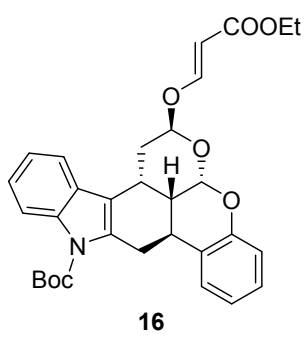

tert-butyl(2S,3aS,3a1S,8bS,14cR)-2-(((E)-3-ethoxy-3-oxoprop-1 -en-1-yl)oxy)-1,3a,3a1,8b,9,14c-hexahydrochromeno[4',3',2':8, 1]isochromeno[6,5-b]indole-10(2H)-carboxylate (16): ${ }^{1} \mathrm{H}$ NMR (400 MHz, $\left.\mathrm{CDCl}_{3}\right) \delta 8.17(\mathrm{~d}, J=7.7 \mathrm{~Hz}, 1 \mathrm{H}), 7.77(\mathrm{~d}, J=12.4 \mathrm{~Hz}, 1 \mathrm{H})$, $7.54-7.48(\mathrm{~m}, 1 \mathrm{H}), 7.37(\mathrm{~d}, J=7.7 \mathrm{~Hz}, 1 \mathrm{H}), 7.34-7.26(\mathrm{~m}, 2 \mathrm{H}), 7.21$ $(\mathrm{t}, J=7.6 \mathrm{~Hz}, 1 \mathrm{H}), 7.07-7.00(\mathrm{~m}, 1 \mathrm{H}), 6.97(\mathrm{dd}, J=8.1,0.8 \mathrm{~Hz}, 1 \mathrm{H}), 5.77(\mathrm{~d}, J=2.5 \mathrm{~Hz}$, 1H), $5.62(\mathrm{~d}, J=12.5 \mathrm{~Hz}, 1 \mathrm{H}), 5.47(\mathrm{~d}, J=3.3 \mathrm{~Hz}, 1 \mathrm{H}), 4.26-4.18(\mathrm{~m}, 2 \mathrm{H}), 4.05(\mathrm{dd}, J=$ 17.6, $5.8 \mathrm{~Hz}, 1 \mathrm{H}$ ), $3.75-3.66(\mathrm{~m}, 1 \mathrm{H}), 3.46$ (td, $J=11.7,5.8 \mathrm{~Hz}, 1 \mathrm{H}), 2.95$ (dd, $J=17.6$, $11.1 \mathrm{~Hz}, 1 \mathrm{H}), 2.40(\mathrm{dd}, J=14.6,5.3 \mathrm{~Hz}, 1 \mathrm{H}), 2.30-2.22(\mathrm{~m}, 1 \mathrm{H}), 1.99-1.87(\mathrm{~m}, 1 \mathrm{H}), 1.72$ (s, 9H), $1.31(\mathrm{t}, J=7.1 \mathrm{~Hz}, 3 \mathrm{H})$ ppm. ${ }^{13} \mathbf{C}$ NMR $\left(100 \mathrm{MHz}, \mathrm{CDCl}_{3}\right) \delta 167.4,158.9,150.7$ 150.5, 136.1, 134.2, 128.1, 127.9, 126.3, 124.1, 123.6, 122.8, 121.9, 119.1, 117.6, 117.0, $115.9,101.3,99.5,91.5,84.0,60.0,35.2,31.3,30.9,28.4,26.3,24.8,14.4$ ppm. HRMS: $[\mathrm{M}+\mathrm{H}]^{+}$calcd. For $\mathrm{C}_{31} \mathrm{H}_{34} \mathrm{NO}_{7}{ }^{+}$532.2330, found 532.2337. $[\alpha]_{\mathrm{D}}{ }^{20}+113.90(c=0.83$ in $\mathrm{CHCl}_{3}$ ). The enantiomeric excess was determined by HPLC analysis on Daicel Chiralpak IC column $[n$-hexane $/ i$-PrOH $=90 / 10,1 \mathrm{~mL} / \mathrm{min}], \lambda=225 \mathrm{~nm}, t_{\text {major }}=47.88 \mathrm{~min}, t_{\text {minor }}=$ $26.39 \mathrm{~min}$, ee $>\mathbf{9 9 \%}$. The diastereomeric ratio was determined by ${ }^{1} \mathrm{H} \mathrm{NMR}, \boldsymbol{d r}>\mathbf{2 0 : 1}$.

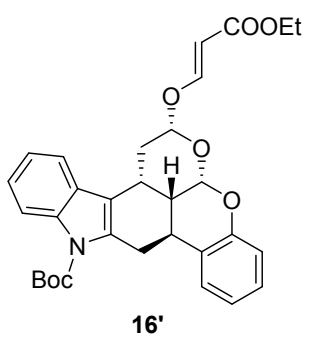

tert-butyl( $2 S, 3 \mathrm{a} S, 3 \mathrm{a} 1 S, 8 \mathrm{~b} S, 14 \mathrm{c} R)-2-(((E)-3$-ethoxy-3-oxoprop1-en-1-yl)oxy)-1,3a,3a1,8b,9,14c-hexahydrochromeno[4',3',2': 8,1]isochromeno[6,5-b]indole-10(2H)-carboxylate $\quad\left(16^{\prime}\right):{ }^{1} \mathrm{H}$ NMR (500 MHz, CDCl $) \delta 8.19(\mathrm{~d}, J=8.2 \mathrm{~Hz}, 1 \mathrm{H}), 7.66(\mathrm{~d}, J=12.5 \mathrm{~Hz}$, 1H), $7.44(\mathrm{~d}, J=7.4 \mathrm{~Hz}, 1 \mathrm{H}), 7.36(\mathrm{~d}, J=7.7 \mathrm{~Hz}, 1 \mathrm{H}), 7.34-7.28(\mathrm{~m}$, 1H), $7.25(\mathrm{~d}, J=8.0 \mathrm{~Hz}, 1 \mathrm{H}), 7.21(\mathrm{t}, J=7.6 \mathrm{~Hz}, 1 \mathrm{H}), 7.03(\mathrm{t}, J=7.5 \mathrm{~Hz}, 1 \mathrm{H}), 6.99-6.96(\mathrm{~m}$, 1H), $5.58(\mathrm{~d}, J=2.2 \mathrm{~Hz}, 1 \mathrm{H}), 5.47(\mathrm{~d}, J=12.5 \mathrm{~Hz}, 1 \mathrm{H}), 5.26(\mathrm{dd}, J=10.1,2.3 \mathrm{~Hz}, 1 \mathrm{H}), 4.22-$ 
$4.14(\mathrm{~m}, 2 \mathrm{H}), 4.06(\mathrm{dd}, J=17.7,5.8 \mathrm{~Hz}, 1 \mathrm{H}), 3.51(\mathrm{td}, J=11.8,5.7 \mathrm{~Hz}, 1 \mathrm{H}), 3.40(\mathrm{dt}, J=$ 12.1, $4.6 \mathrm{~Hz}, 1 \mathrm{H}$ ), 2.95 (dd, $J=17.5,11.1 \mathrm{~Hz}, 1 \mathrm{H}$ ), 2.50 (ddd, $J=13.7,5.0,2.3 \mathrm{~Hz}, 1 \mathrm{H}$ ), 2.21 (ddd, $J=12.7,4.5,2.5 \mathrm{~Hz}, 1 \mathrm{H}), 1.83(\mathrm{td}, J=13.0,10.2 \mathrm{~Hz}, 1 \mathrm{H}), 1.74(\mathrm{~s}, 9 \mathrm{H}), 1.29(\mathrm{t}, J=7.1$ $\mathrm{Hz}, 3 \mathrm{H}) \mathrm{ppm} .{ }^{13} \mathrm{C}$ NMR $\left(100 \mathrm{MHz}, \mathrm{CDCl}_{3}\right) \delta 167.2,159.2,150.8,150.4,136.2,134.4,128.1$, $127.8,126.3,124.1,123.5,122.8,122.0,117.9,117.3,116.9,115.9,101.3,100.0,95.5$, 84.0, 60.0, 35.19, 33.1, 30.7, 29.1, 28.4, 25.5, 14.4 ppm. HRMS: $[\mathrm{M}+\mathrm{H}]^{+}$calcd. For $\mathrm{C}_{31} \mathrm{H}_{34} \mathrm{NO}_{7}+532.2330$, found 532.2337. $[\alpha]_{\mathbf{D}}{ }^{20}-29.31\left(c=0.92\right.$ in $\left.\mathrm{CHCl}_{3}\right)$. The enantiomeric excess was determined by HPLC analysis on Daicel Chiralpak IC column $[n$-hexane $/ i-\mathrm{PrOH}=90 / 10,1 \mathrm{~mL} / \mathrm{min}], \lambda=220 \mathrm{~nm}, t_{\text {major }}=52.35 \mathrm{~min}, t_{\text {minor }}=38.43 \mathrm{~min}$, ee $>\mathbf{9 9 \%}$. The diastereomeric ratio was determined by ${ }^{1} \mathrm{H} N \mathrm{NR}, \boldsymbol{d r}>\mathbf{2 0 : 1}$. 


\section{G. 1 mmol scale reaction}

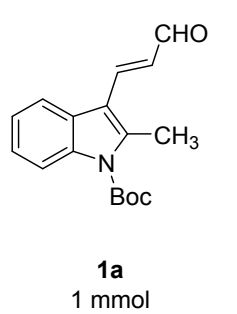<smiles>O=C/C=C/c1ccccc1O</smiles>
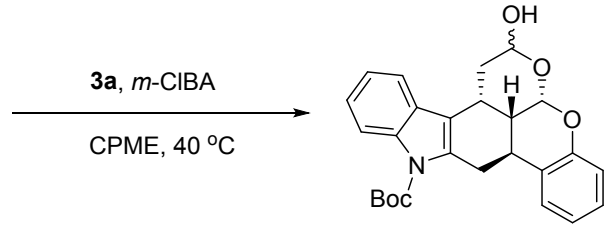

$1 \mathrm{mmol}$

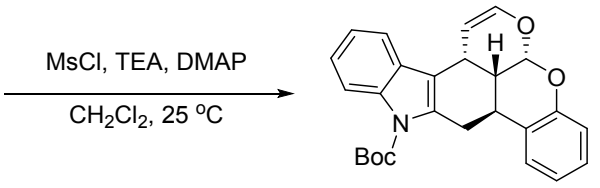

$6 a$

Detailed method: A glass vial equipped with a magnetic stirring bar was charged with catalyst 3a (0.2 mmol, $20 \mathrm{~mol} \%)$ in CPME (2 mL), then 2-hydroxycinnamaldehyde 2a (1.2 mmol, 1.2 equiv.), 3-chlorobenzoic acid ( $0.2 \mathrm{mmol}, 20 \mathrm{~mol} \%)$ and the aldehyde $1 \mathrm{a}$ (1.0 mmol, 1 equiv.) was added in one portion and the resulting solution was stirred for about $11 \mathrm{~h}$ at $40^{\circ} \mathrm{C}$ in oil bath until the material 1a disappeared. After completion of the reaction, pure compound was filtered by ether to collect white solids $256 \mathbf{m g}$. The $\mathbf{5 a}$ product (1.0 equiv.) was respectively dissolved in $\mathrm{CH}_{2} \mathrm{Cl}_{2}(0.2 \mathrm{M})$ at $0{ }^{\circ} \mathrm{C}$ in ice bath. TEA (6.0 equiv.), $\mathrm{MsCl}$ (2.0 equiv.), DMAP (0.2 equiv.) were added to the reaction mixtures. After full conversion of the reaction, the reaction mixture was purified by flash chromatography on silica gel (petroleum ether/ethyl acetate $=90 / 1$ ) to give product $\mathbf{6 a}$ as white solid $190 \mathrm{mg}$ for $46 \%$ yield and ee $\mathbf{8 9 9 \%}$. The diastereomeric ratio was determined by ${ }^{1} \mathrm{H}$ NMR, $\boldsymbol{d} \boldsymbol{r}>\mathbf{2 0 : 1}$. 


\section{H. Control Experiments}

\section{H1. Nonlinear effect study}

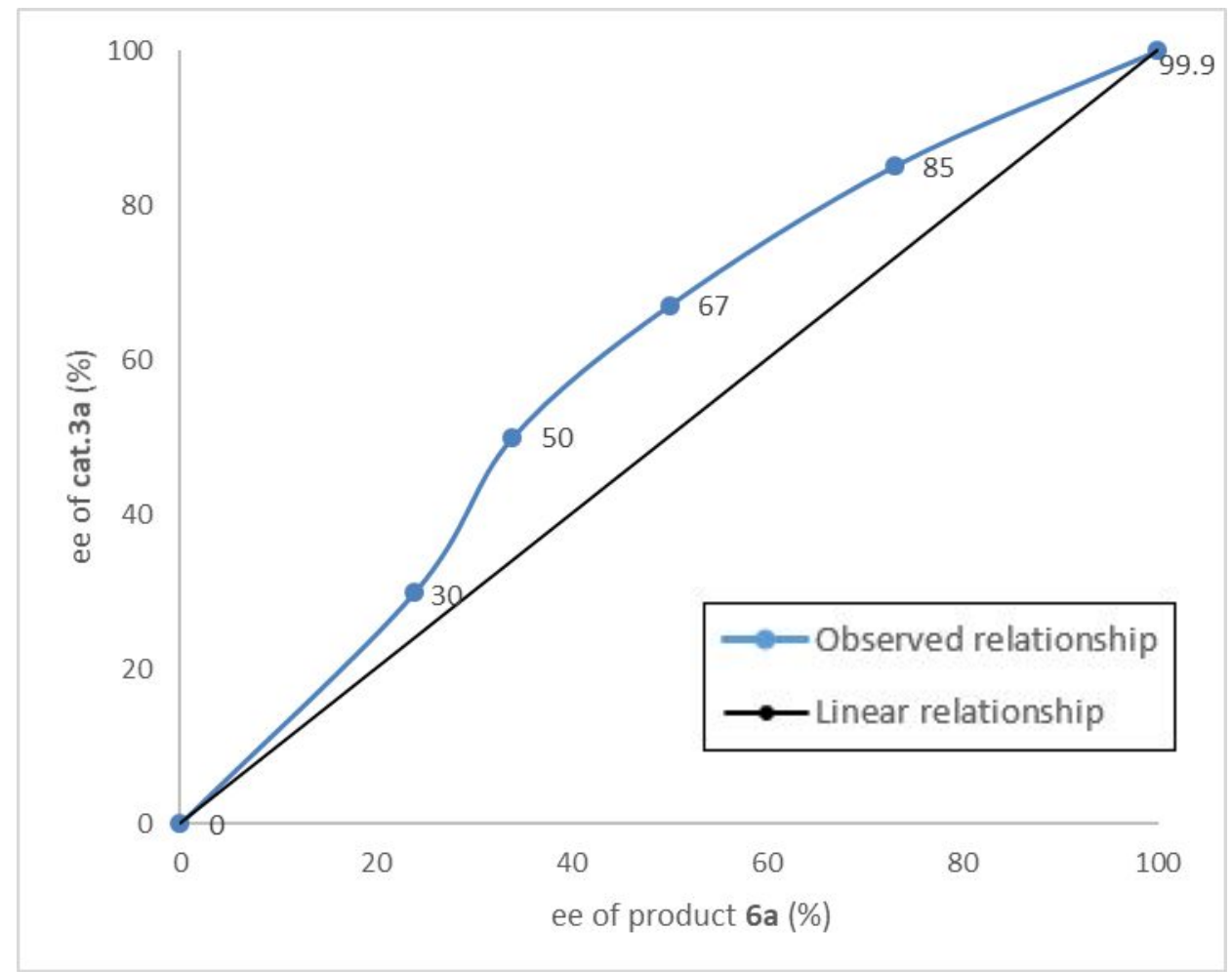

Figure 1: Nonlinear effect study of the product $6 \mathbf{a}$ and cat.3a with different ee values.

\section{H2. Substrates by using -CN group instead of -CHO group}

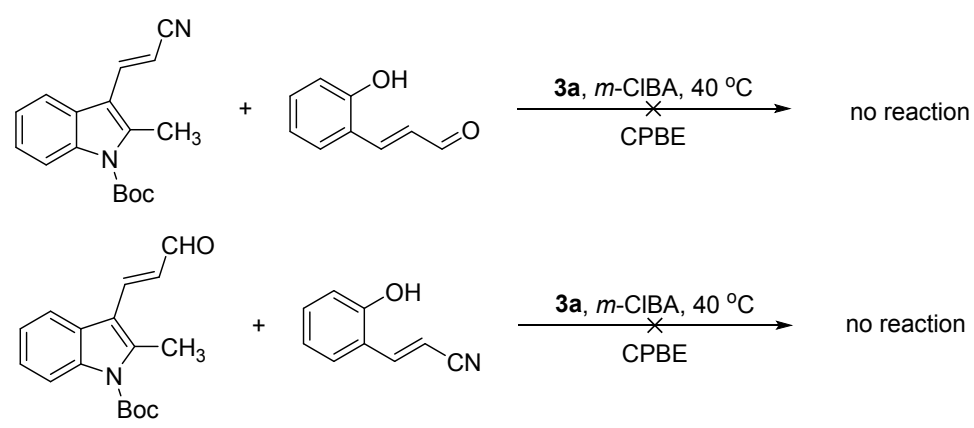

Two cyano-substituted compounds did not react under our conditions. 


\section{Unreactive substituted 2,4-dienals}

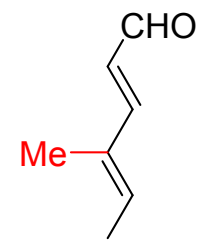

4-methyl-substituted

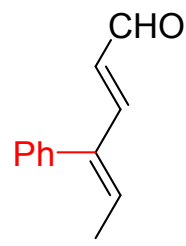

4-phenyl-substituted

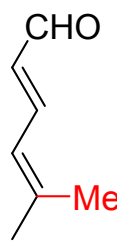

5-methyl-substituted

Unfortunately, under our conditions, almost no reaction was observed with 4-methyl-, 4-phenyl- and 5-methyl-substituted 2,4-dienals, 


\section{J. NMR spectra and HPLC traces}

The ${ }^{1} \mathrm{H}$ NMR spectrum of $6 \mathrm{a}\left(500 \mathrm{MHz}, \mathrm{CDCl}_{3}\right)$

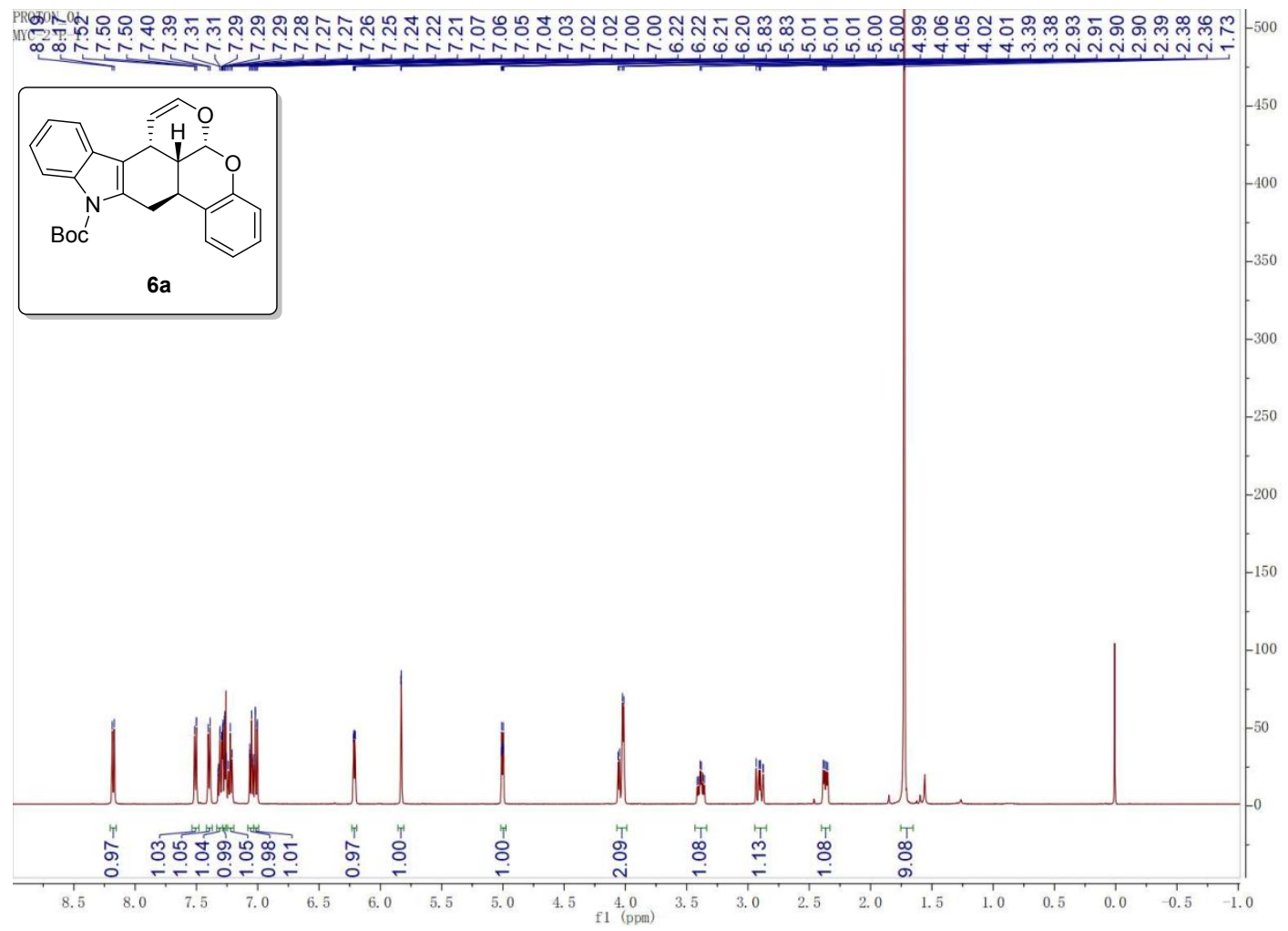

The ${ }^{13} \mathrm{C}$ NMR spectrum of $6 \mathrm{a}\left(125 \mathrm{MHz}, \mathrm{CDCl}_{3}\right)$

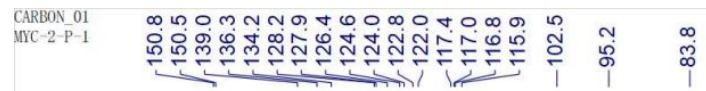
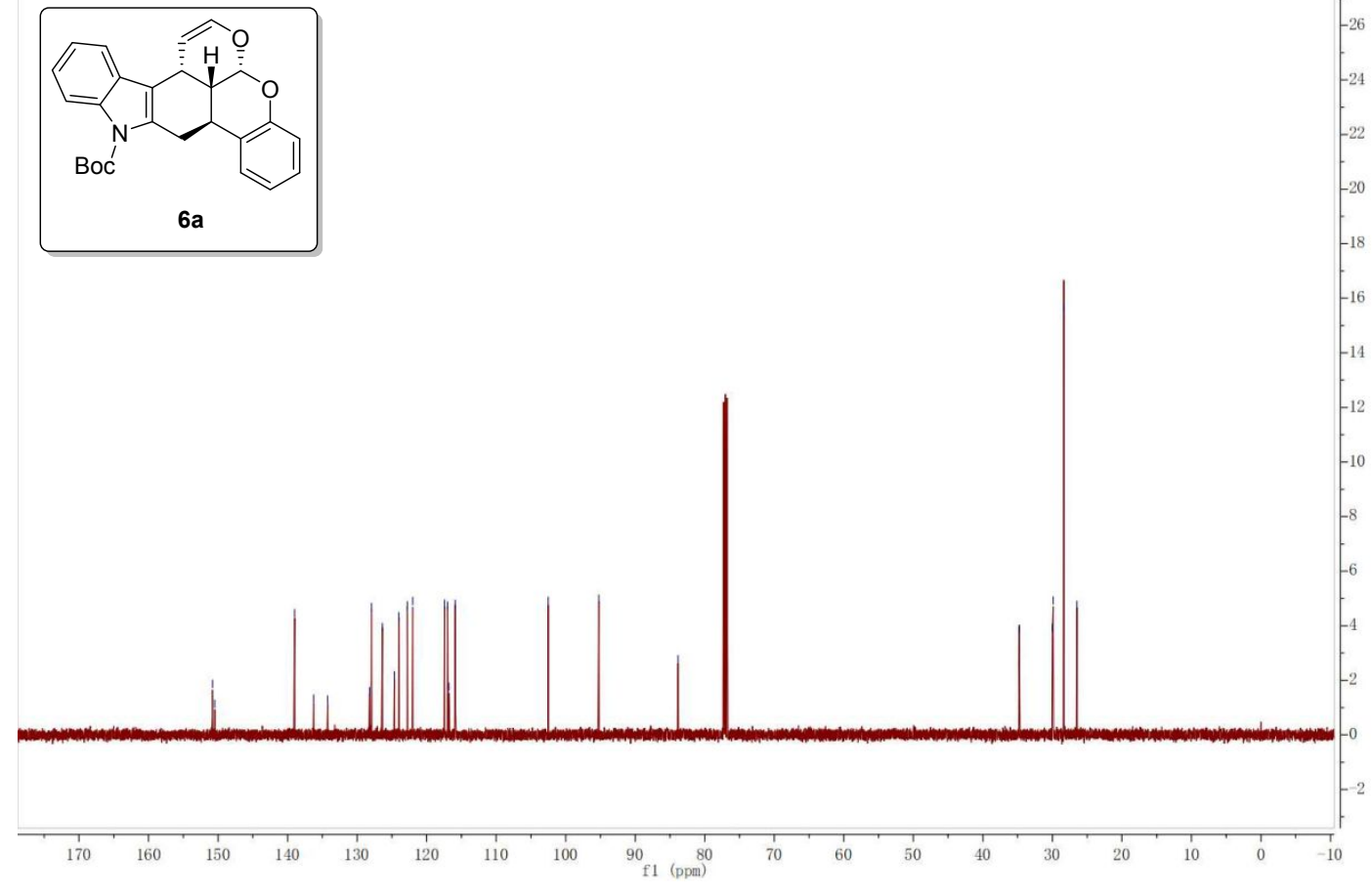


\section{The HPLC of racemic $6 a$}

Chrom Type: Fixed WL Chromatogram, 220 nm

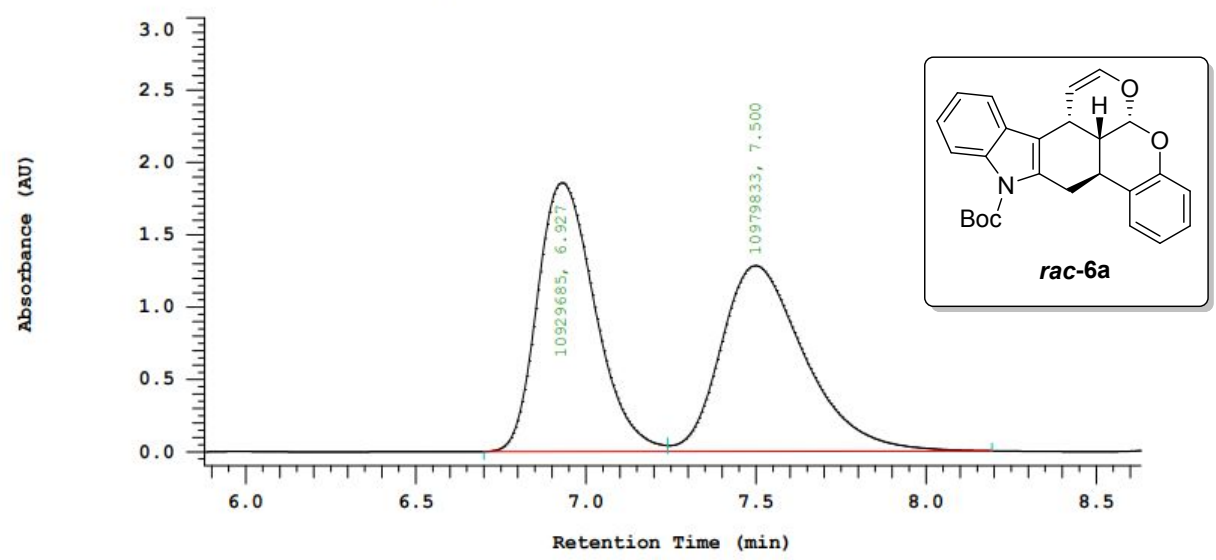

Chrom Type: Fixed WL Chromatogram, $220 \mathrm{~nm}$

Peak Quantitation: AREA

Calculation Method: AREA응

\begin{tabular}{|c|c|c|c|c|}
\hline No. & $\mathrm{RT}$ & Area & Area \% & $\mathrm{BC}$ \\
\hline 1 & 6.927 & 10929685 & 49.886 & BV \\
\hline \multirow[t]{2}{*}{2} & 7.500 & 10979833 & 50.114 & VB \\
\hline & & 21909518 & 100.000 & \\
\hline
\end{tabular}

The HPLC of chiral $6 a$

Chrom Type: Fixed WL Chromatogram, $220 \mathrm{~nm}$

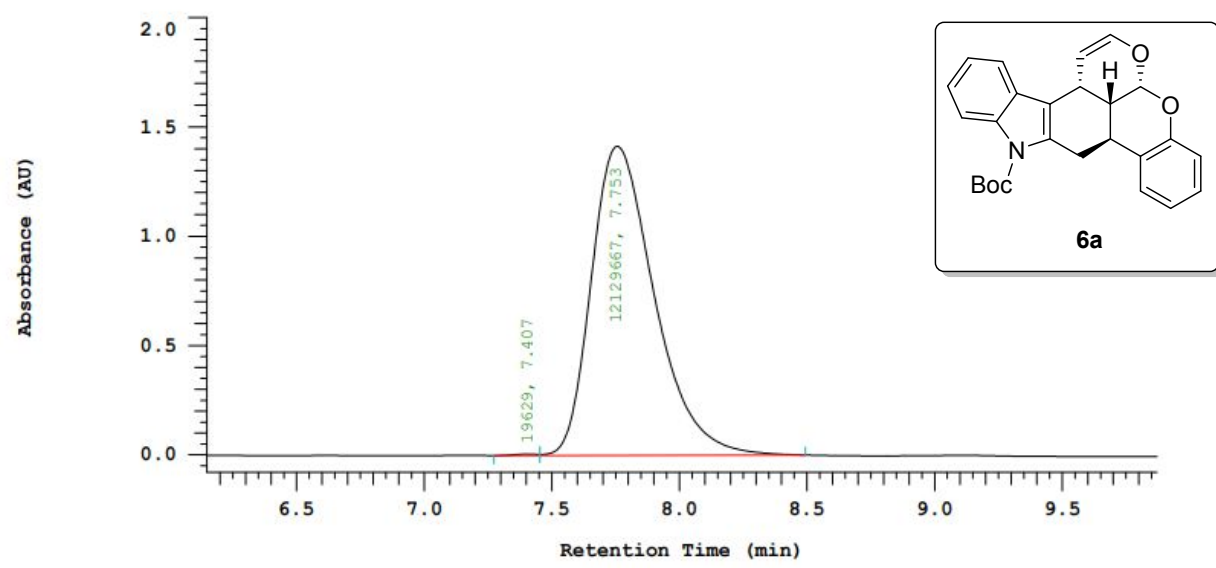

Chrom Type: Fixed WL Chromatogram, $220 \mathrm{~nm}$

Peak Quantitation: AREA

Calculation Method: AREA응

\begin{tabular}{|c|c|c|c|c|}
\hline No. & $\mathrm{RT}$ & Area & Area $\frac{\circ}{\circ}$ & $\mathrm{BC}$ \\
\hline 1 & 7.407 & 19629 & 0.162 & BV \\
\hline 2 & 7.753 & 12129667 & 99.838 & VB \\
\hline & & 12149296 & 100.000 & \\
\hline
\end{tabular}


The ${ }^{1} \mathrm{H}$ NMR spectrum of $6 \mathrm{~b}\left(400 \mathrm{MHz}, \mathrm{CDCl}_{3}\right)$

MYC-2-20-2

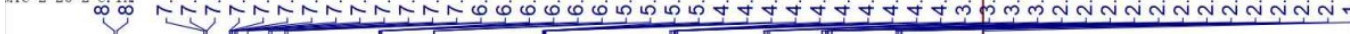
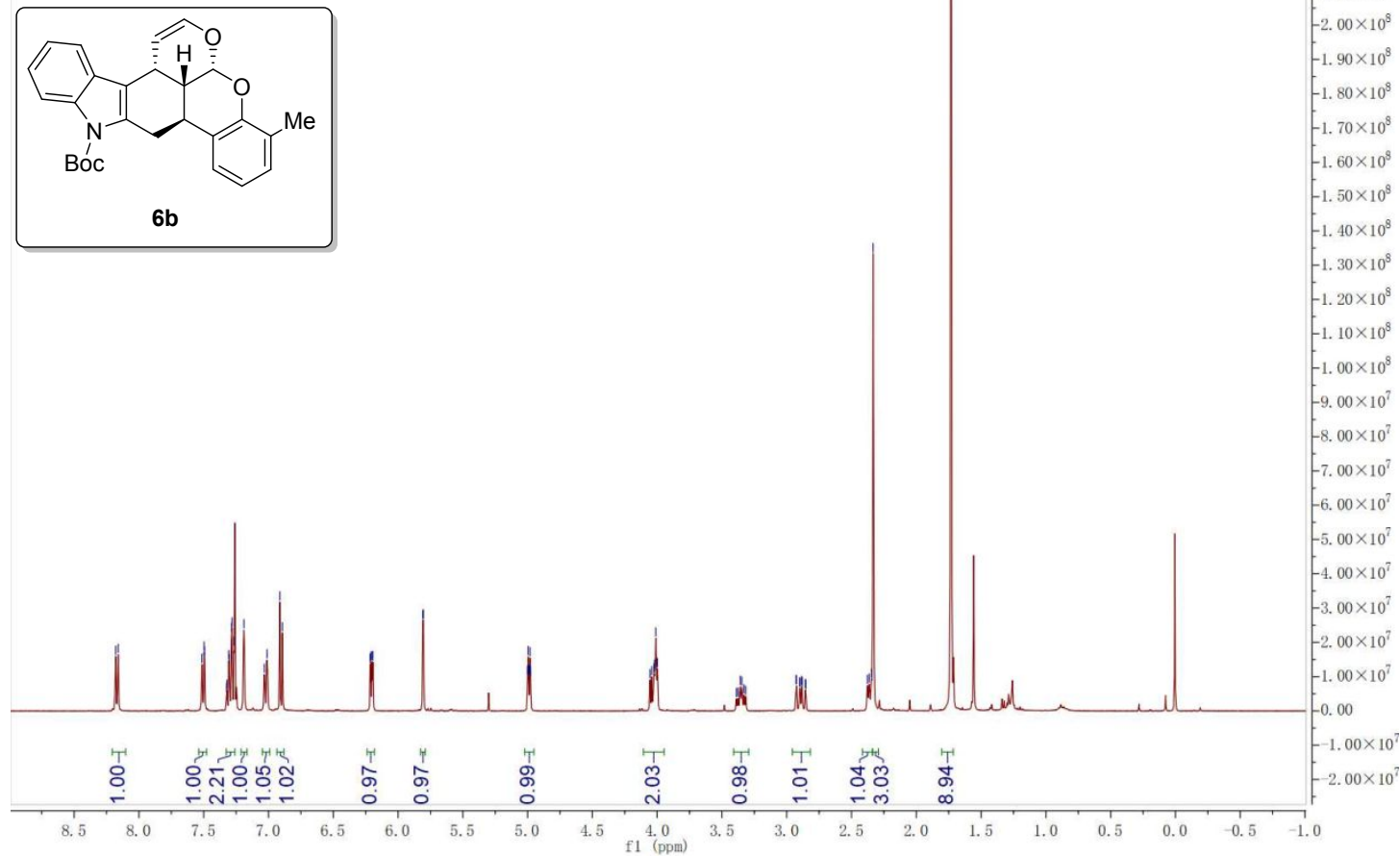

The ${ }^{13} \mathrm{C}$ NMR spectrum of $6 \mathrm{~b}\left(100 \mathrm{MHz}, \mathrm{CDCl}_{3}\right)$

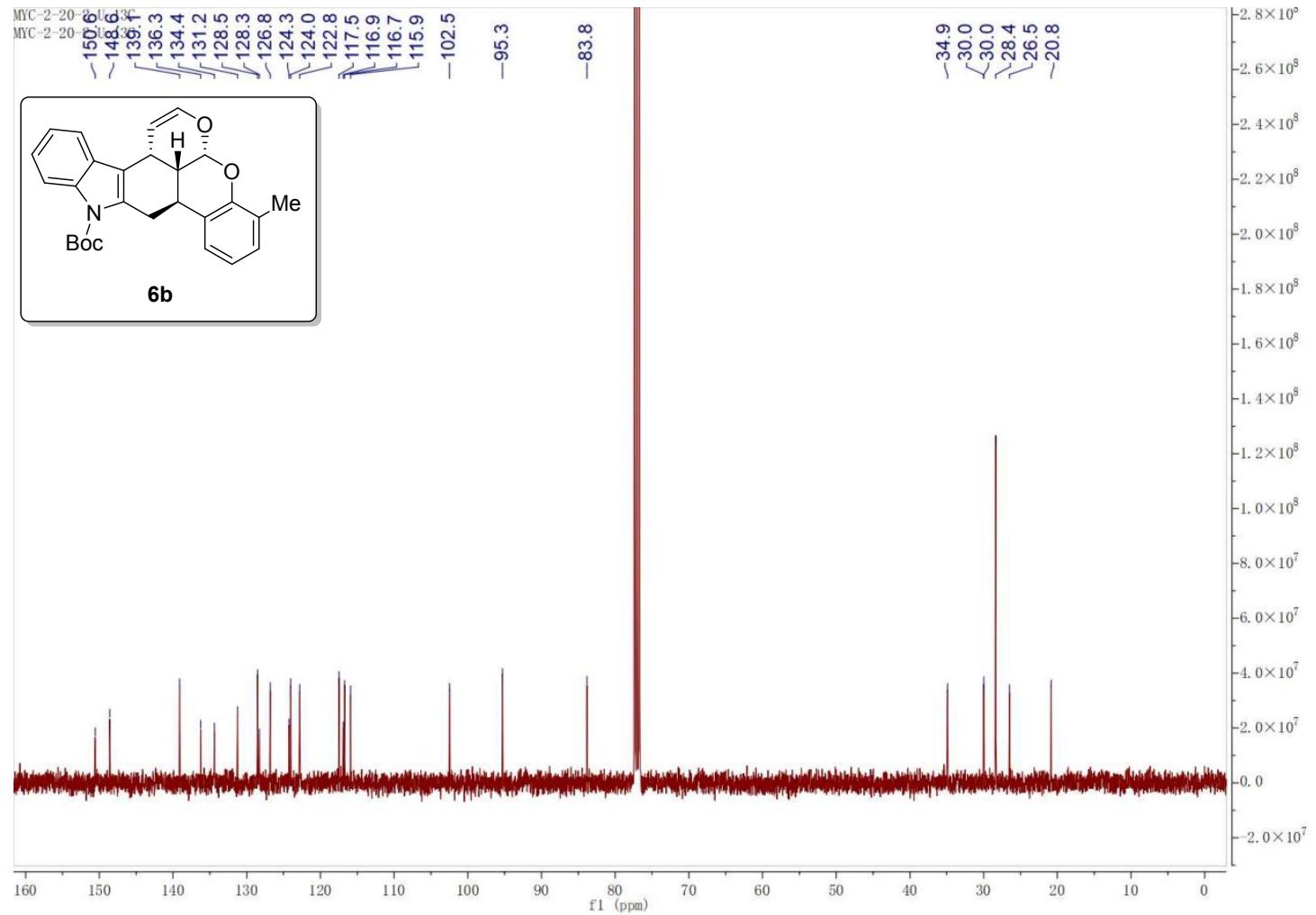




\section{The HPLC of racemic $6 b$}

Chrom Type: Fixed WL Chromatogram, 220 nm

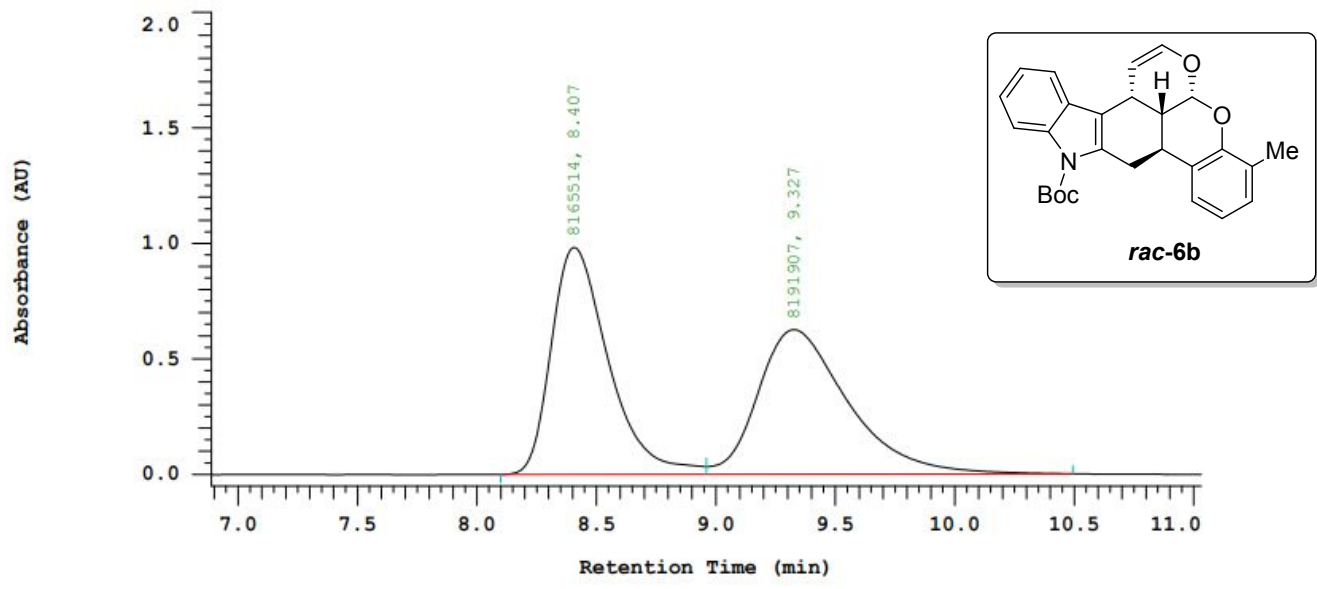

Chrom Type: Fixed WL Chromatogram, $220 \mathrm{~nm}$

Peak Quantitation: AREA

Calculation Method: AREA용

\begin{tabular}{ccccc} 
No. & RT & Area & Area & BC \\
\hline 1 & 8.407 & 8165514 & 49.919 & BV \\
2 & 9.327 & 8191907 & 50.081 & VB \\
\hline & & 16357421 & 100.000 \\
\hline
\end{tabular}

\section{The HPLC of chiral $6 b$}

Chrom Type: Fixed WL Chromatogram, 220 nm

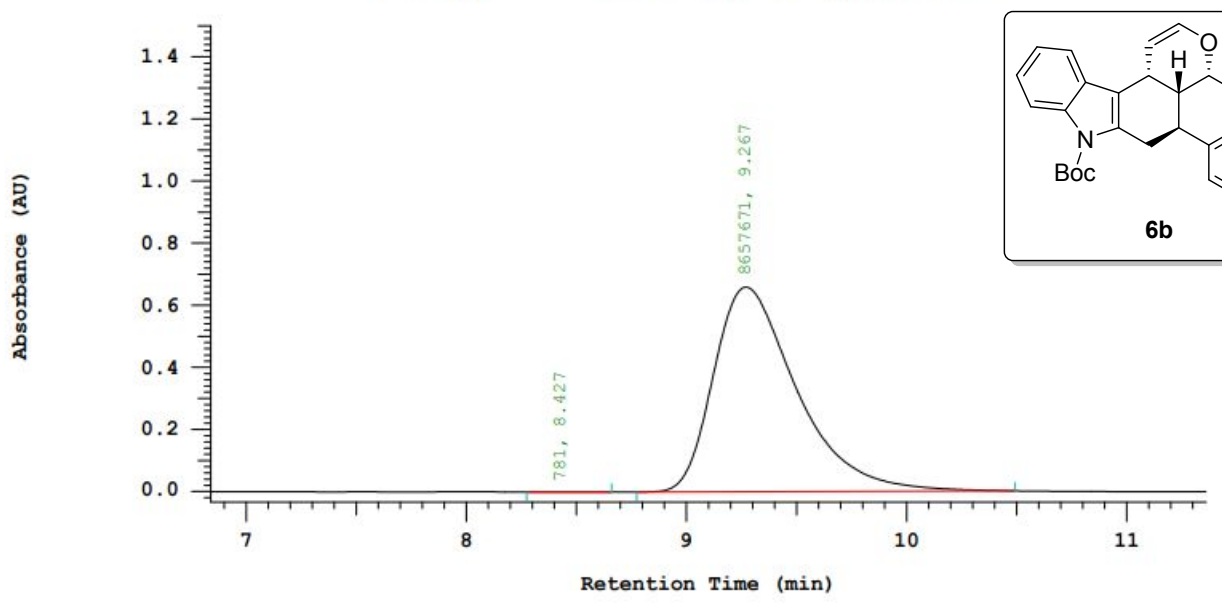

Chrom Type: Fixed WL Chromatogram, $220 \mathrm{~nm}$

Peak Quantitation: AREA

Calculation Method: AREA응

\begin{tabular}{ccccc} 
No. & RT & Area & Area & BC \\
\hline 1 & 8.427 & 781 & 0.009 & BB \\
2 & 9.267 & 8657671 & 99.991 & BB \\
\hline & & 8658452 & 100.000 &
\end{tabular}


The ${ }^{1} \mathrm{H}$ NMR spectrum of $6 \mathrm{c}\left(400 \mathrm{MHz}, \mathrm{CDCl}_{3}\right)$

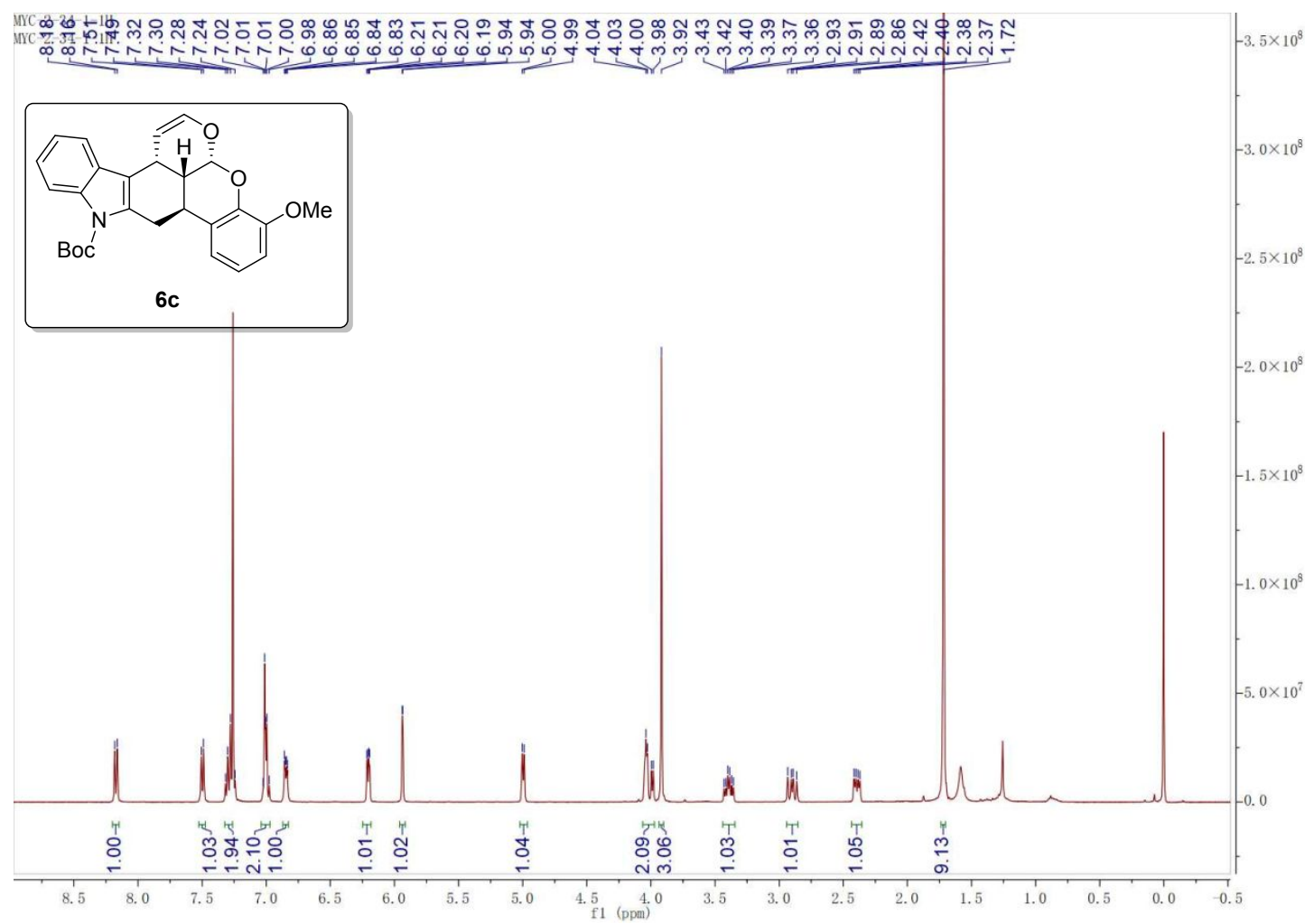

The ${ }^{13} \mathrm{C}$ NMR spectrum of $6 \mathrm{c}\left(100 \mathrm{MHz}, \mathrm{CDCl}_{3}\right)$

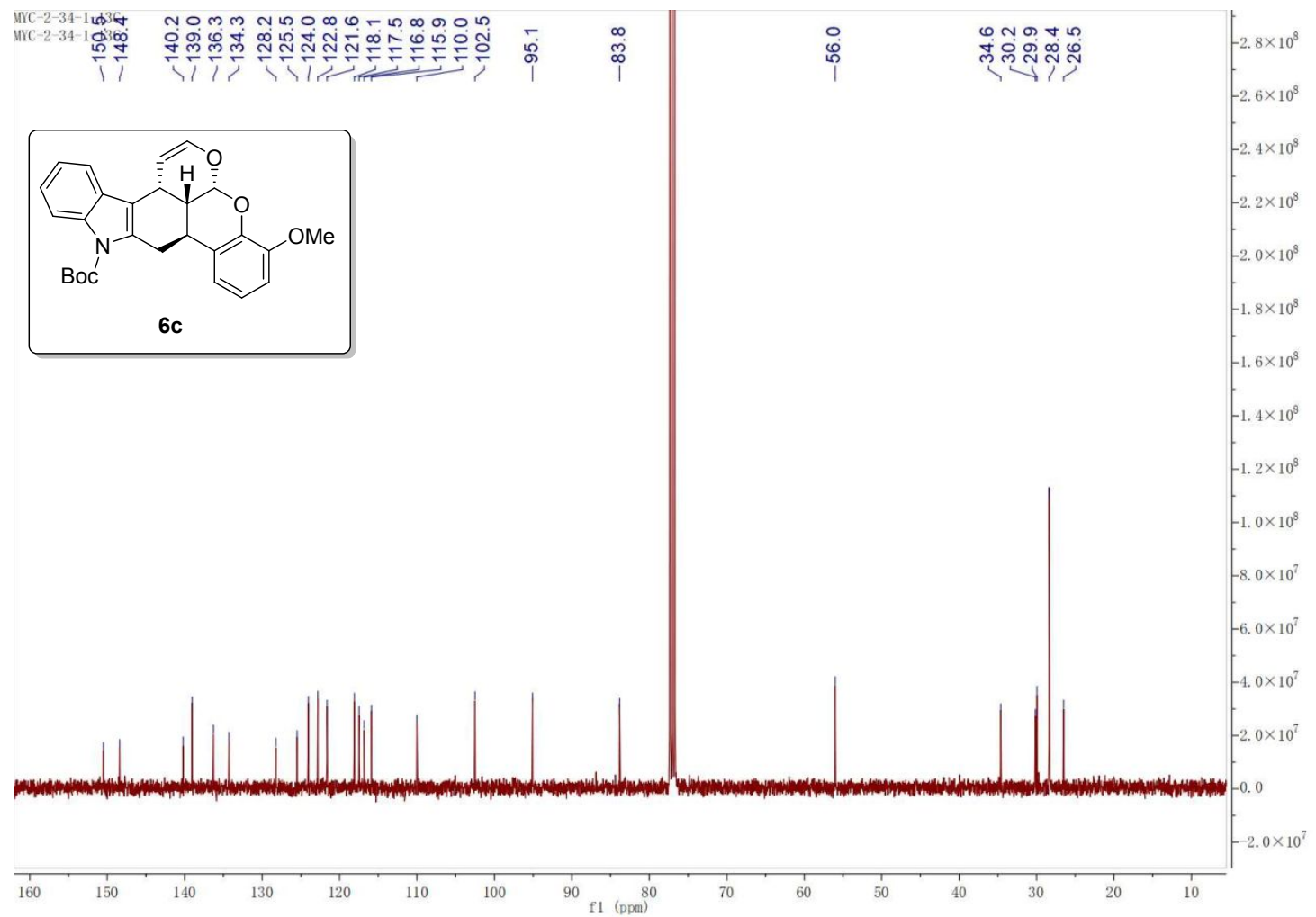




\section{The HPLC of racemic $6 c$}

Chrom Type: Fixed WL Chromatogram, 205 nm

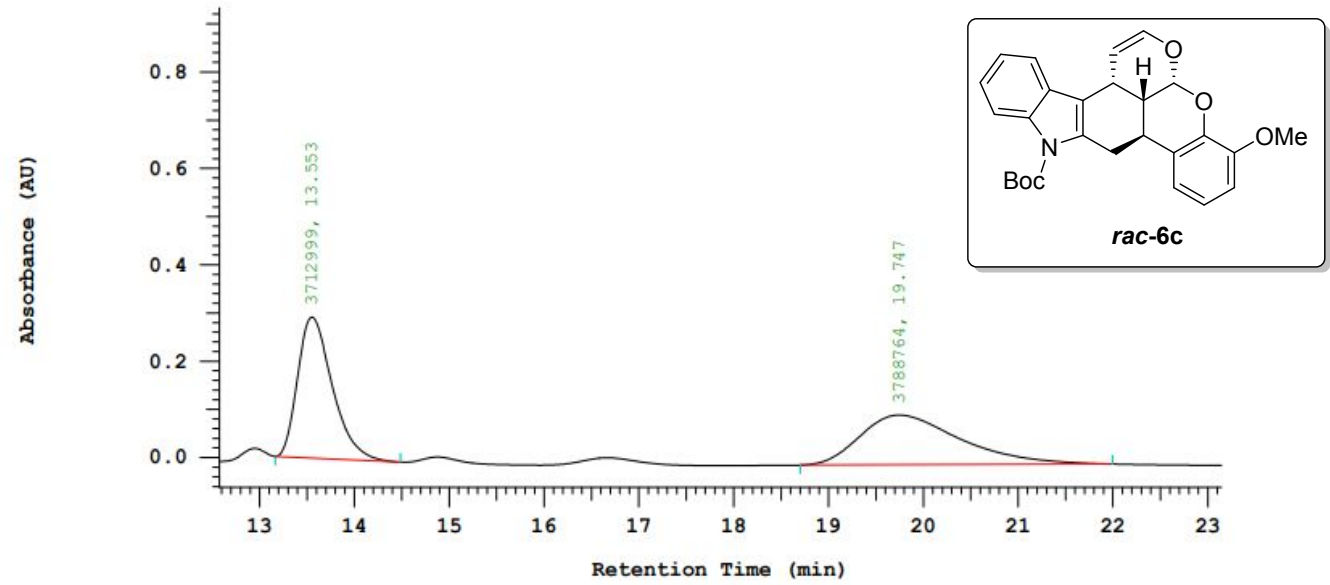

Chrom Type: Fixed WL Chromatogram, $205 \mathrm{~nm}$

Peak Quantitation: AREA

Calculation Method: AREA용

\begin{tabular}{ccccc} 
No. & RT & Area & Area & BC \\
\hline 1 & 13.553 & 3712999 & 49.495 & BB \\
2 & 19.747 & 3788764 & 50.505 & BB \\
\hline & & 7501763 & 100.000 & \\
\hline
\end{tabular}

\section{The HPLC of chiral 6c}

Chrom Type: Fixed WL Chromatogram, 205 nm

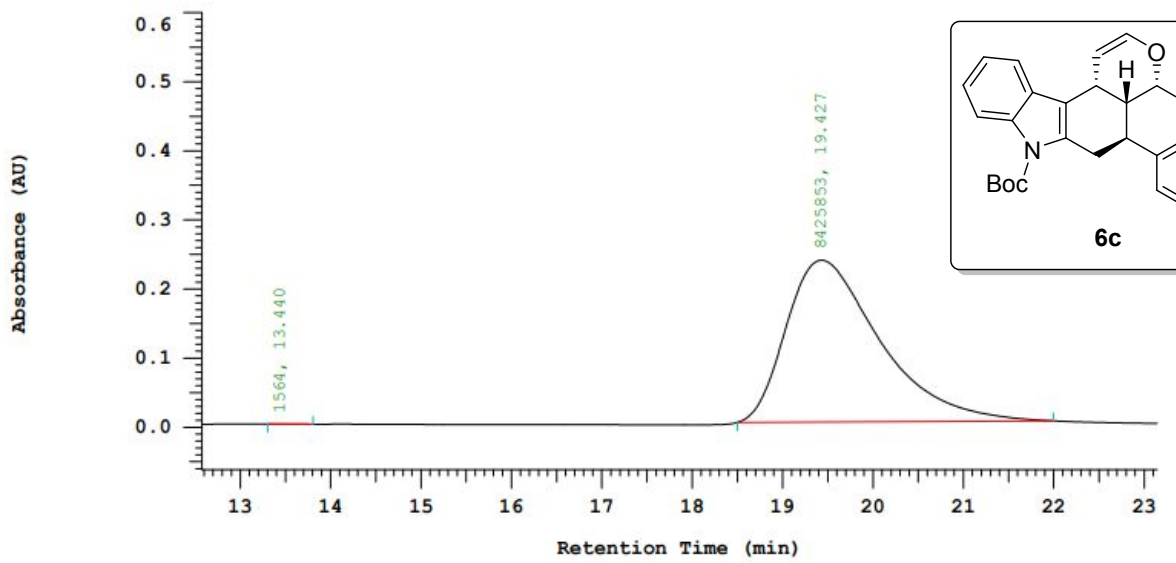

Chrom Type: Fixed WL Chromatogram, $205 \mathrm{~nm}$

Peak Quantitation: AREA

Calculation Method: AREA용

\begin{tabular}{|c|c|c|c|c|}
\hline No. & $\mathrm{RT}$ & Area & Area $\frac{8}{6}$ & $\mathrm{BC}$ \\
\hline 1 & 13.440 & 1564 & 0.019 & $\mathrm{BB}$ \\
\hline \multirow[t]{2}{*}{2} & 19.427 & 8425853 & 99.981 & $\mathrm{BB}$ \\
\hline & & 8427417 & 100.000 & \\
\hline
\end{tabular}


The ${ }^{1} \mathrm{H}$ NMR spectrum of $6 \mathrm{~d}\left(400 \mathrm{MHz}, \mathrm{CDCl}_{3}\right)$

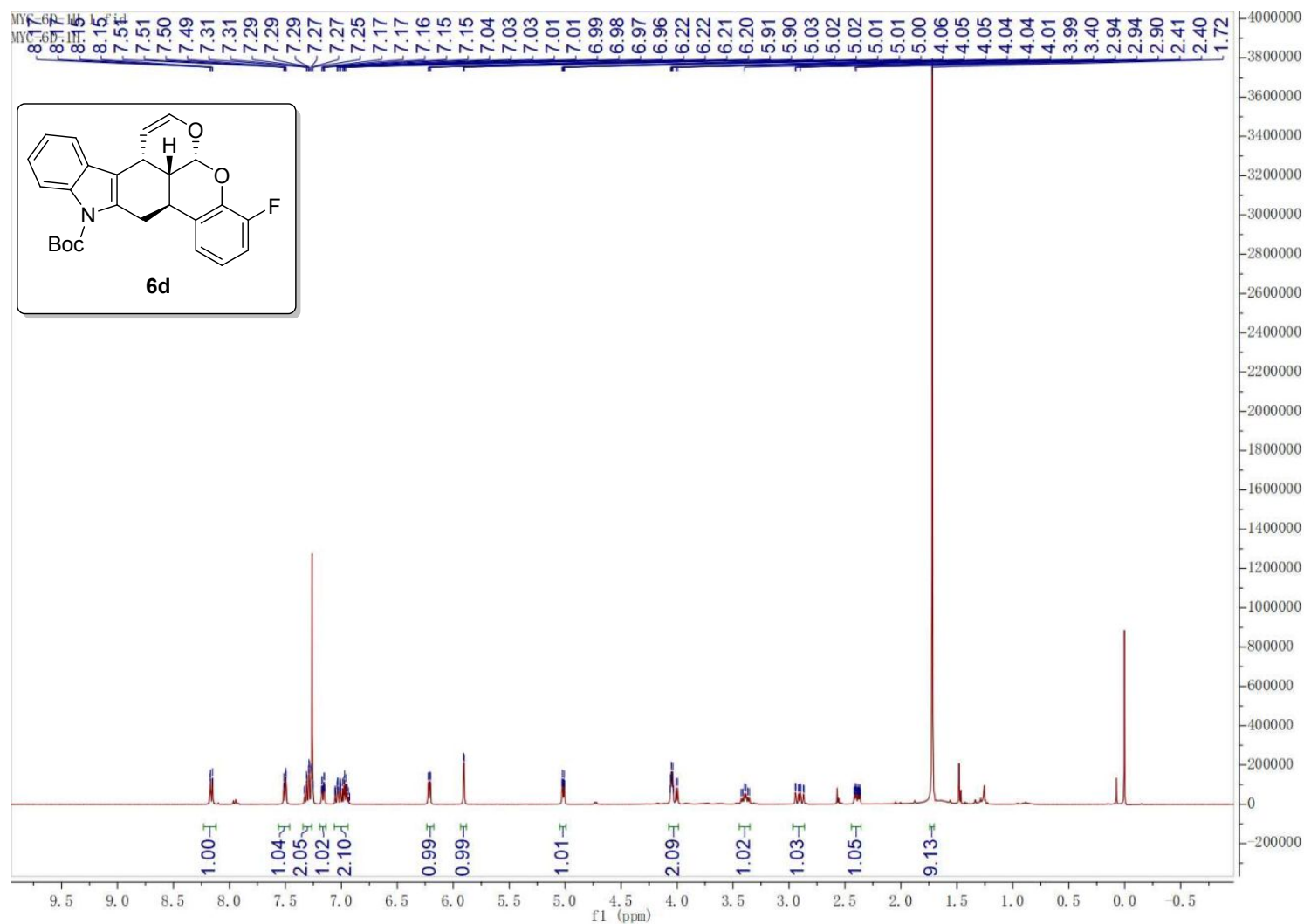

The ${ }^{13} \mathrm{C}$ NMR spectrum of $6 \mathrm{~d}\left(100 \mathrm{MHz}, \mathrm{CDCl}_{3}\right)$
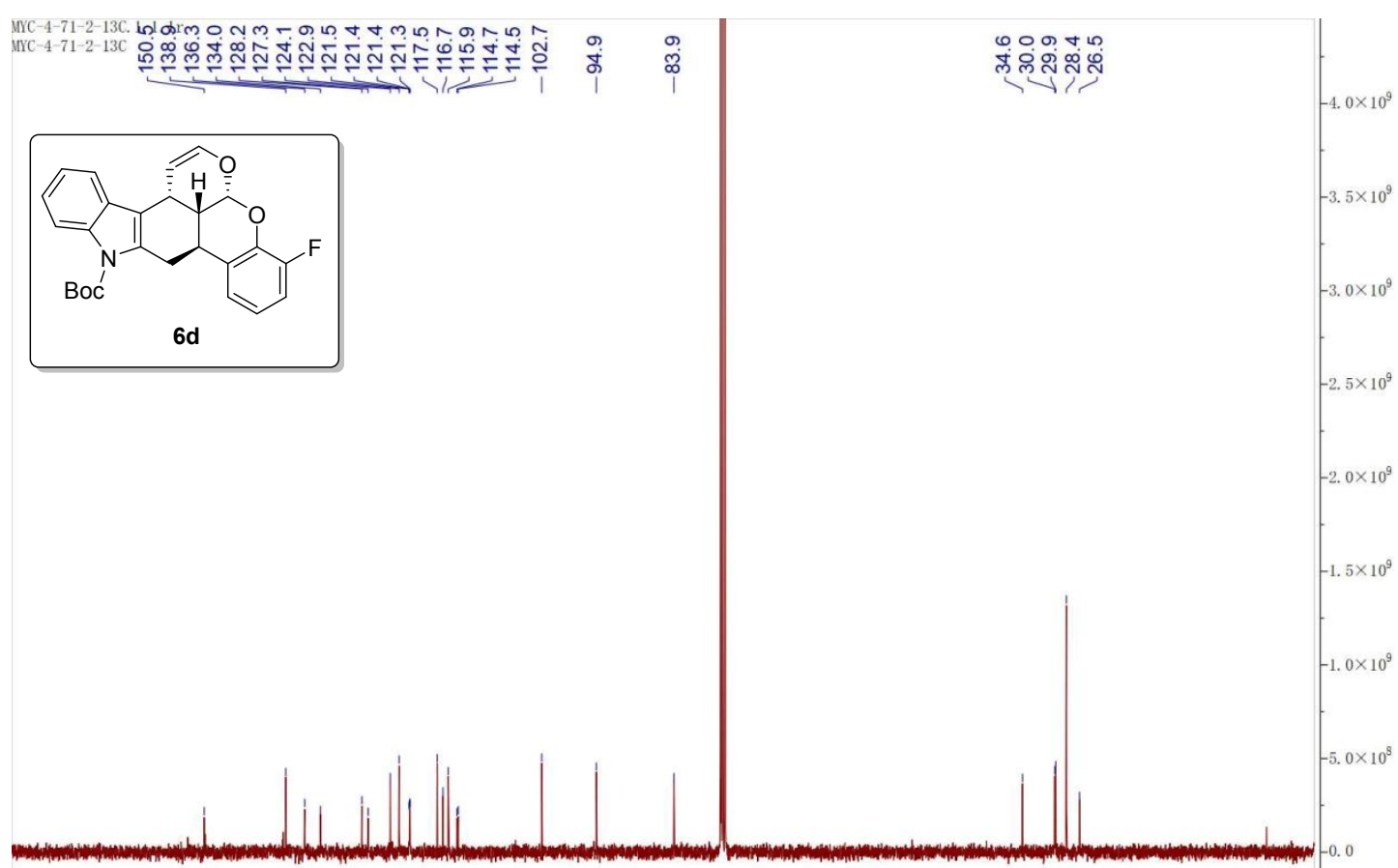

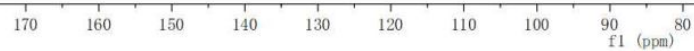




\section{The HPLC of racemic $6 d$}

Chrom Type: Fixed WL Chromatogram, 220 nm

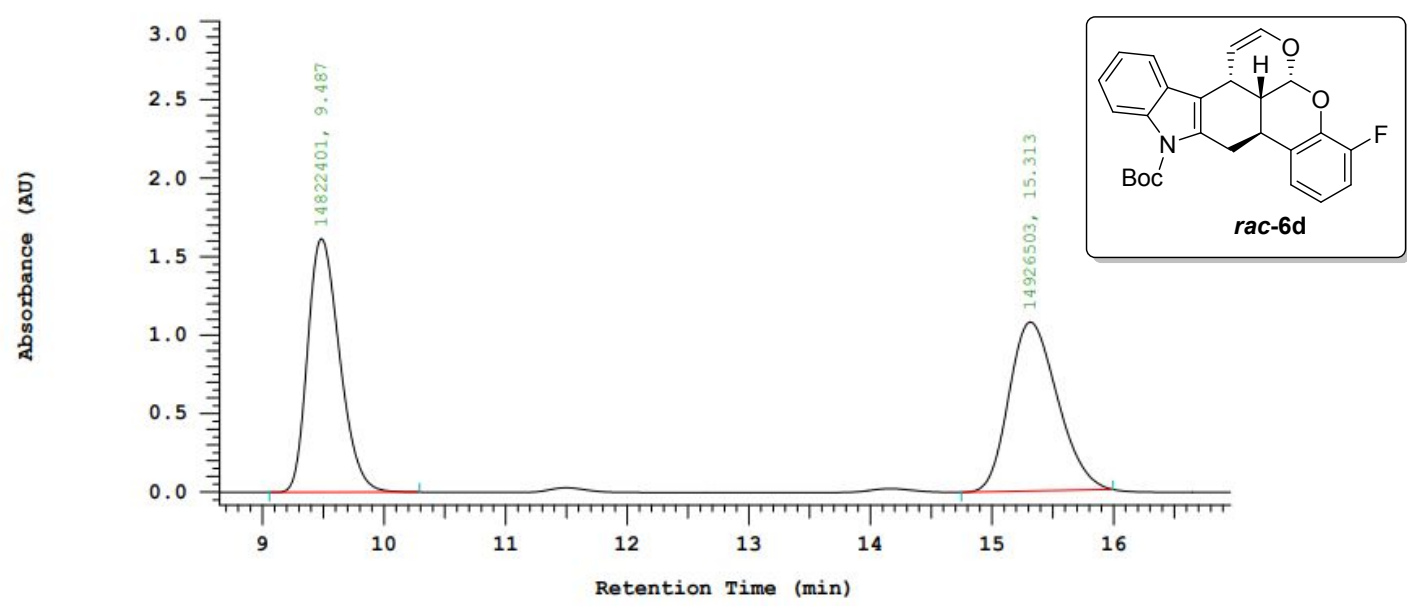

Chrom Type: Fixed WL Chromatogram, 220 nm

Peak Quantitation: AREA

Calculation Method: AREA음

\begin{tabular}{|c|c|c|c|c|}
\hline No. & $\mathrm{RT}$ & Area & Area $\frac{8}{\partial}$ & $\mathrm{BC}$ \\
\hline 1 & 9.487 & 14822401 & 49.825 & $\mathrm{BB}$ \\
\hline 2 & 15.313 & 14926503 & 50.175 & $\mathrm{BB}$ \\
\hline & & 29748904 & 100.000 & \\
\hline
\end{tabular}

\section{The HPLC of chiral 6d}

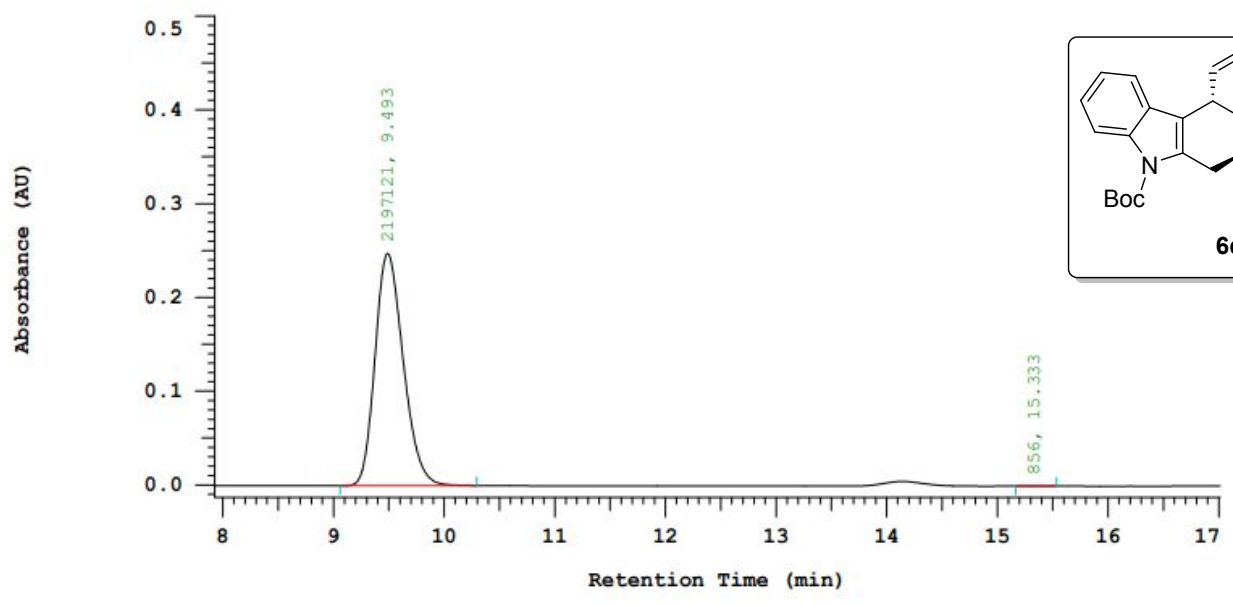

Chrom Type: Fixed WL Chromatogram, $220 \mathrm{~nm}$

Peak Quantitation: AREA

Calculation Method: AREA응

\begin{tabular}{rrrrr} 
No. & \multicolumn{1}{c}{ RT } & Area & Area $~$ & BC \\
\hline 1 & 9.493 & 2197121 & 99.961 & BB \\
2 & 15.333 & 856 & 0.039 & BB \\
\hline & & 2197977 & 100.000 & \\
\hline
\end{tabular}


The ${ }^{1} \mathrm{H}$ NMR spectrum of $6 \mathrm{e}\left(400 \mathrm{MHz}, \mathrm{CDCl}_{3}\right)$

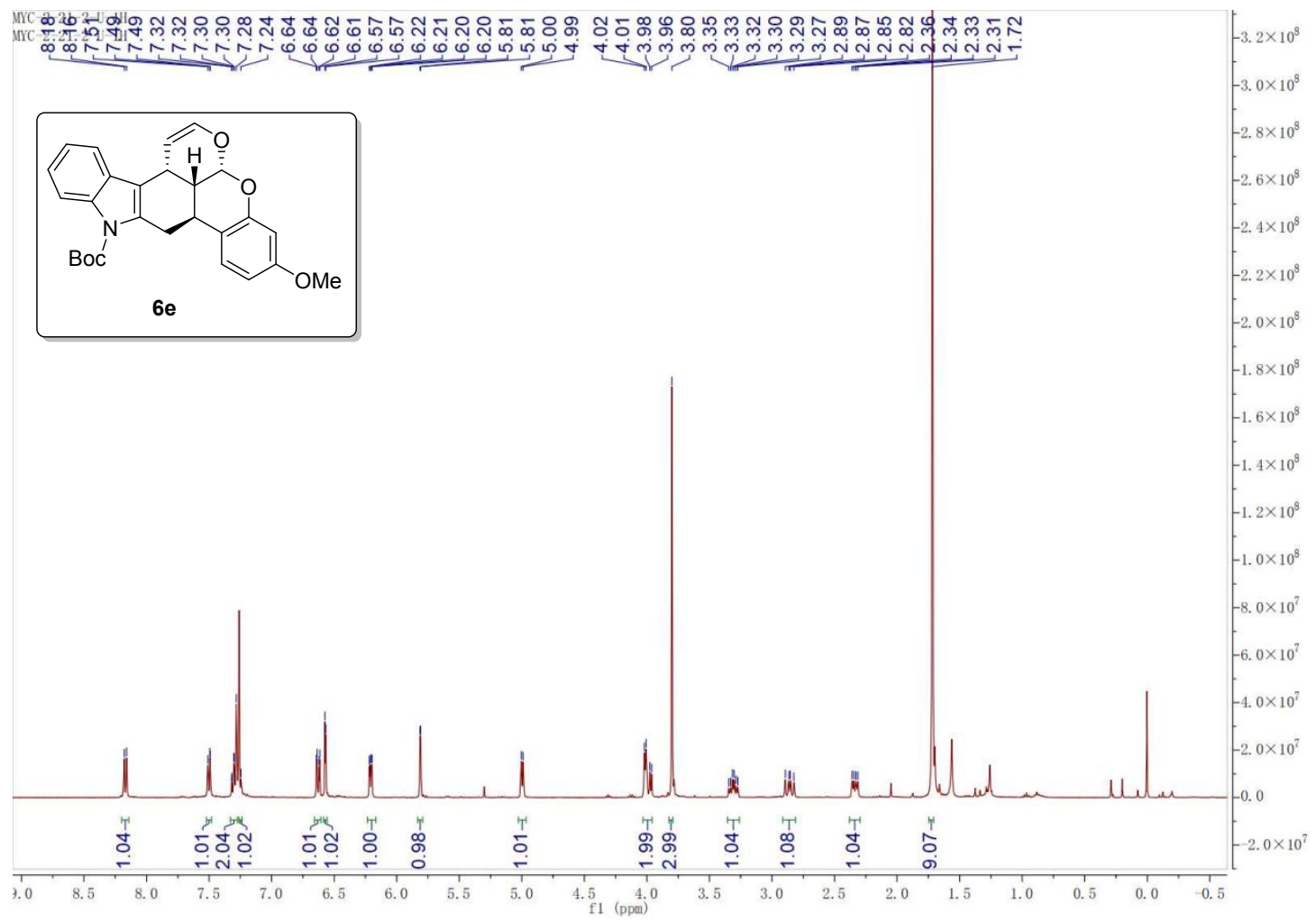

The ${ }^{13} \mathrm{C}$ NMR spectrum of $6 \mathrm{e}\left(100 \mathrm{MHz}, \mathrm{CDCl}_{3}\right)$

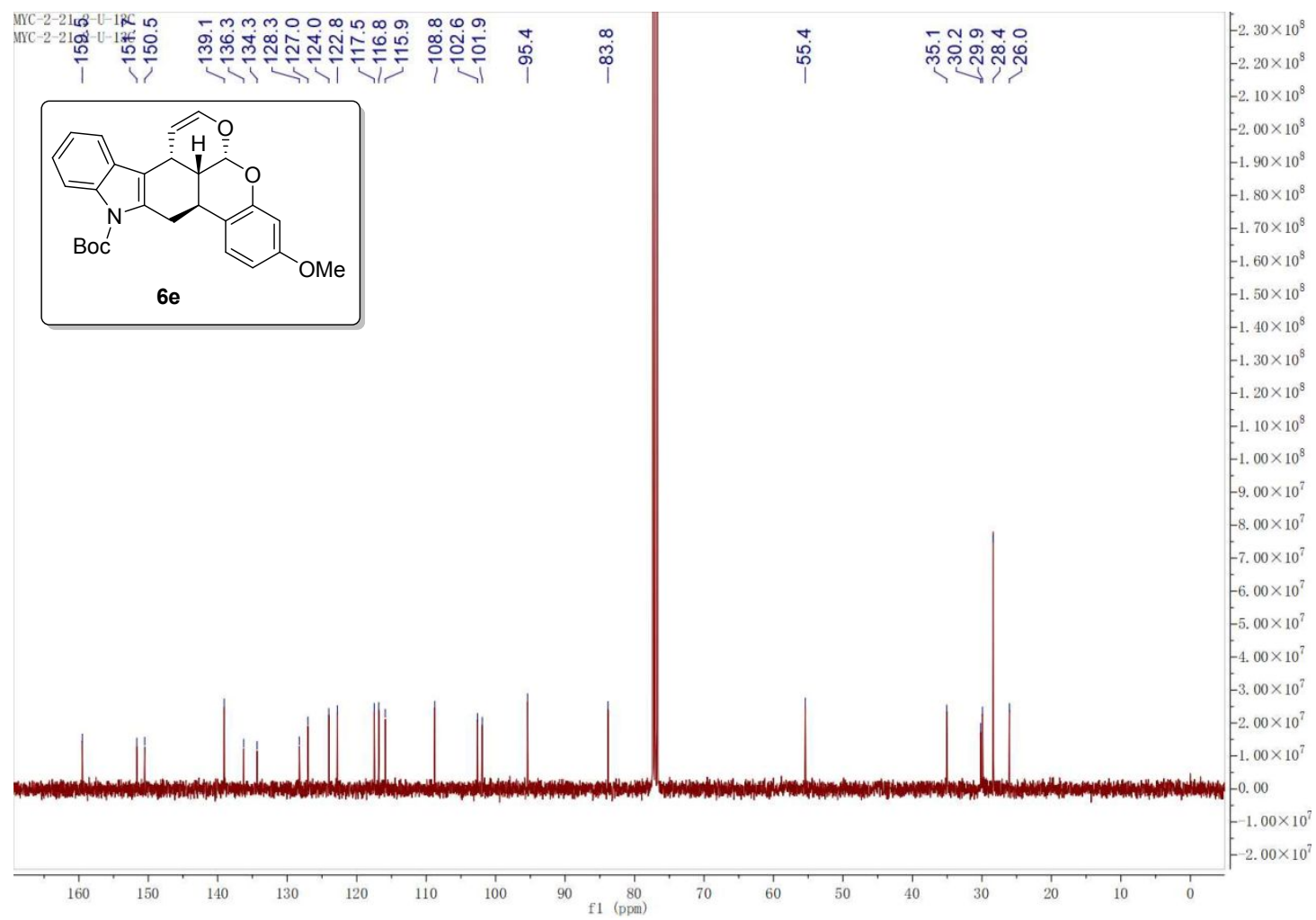


The HPLC of racemic $6 \mathrm{e}$

Chrom Type: Fixed WL Chromatogram, 205 nm

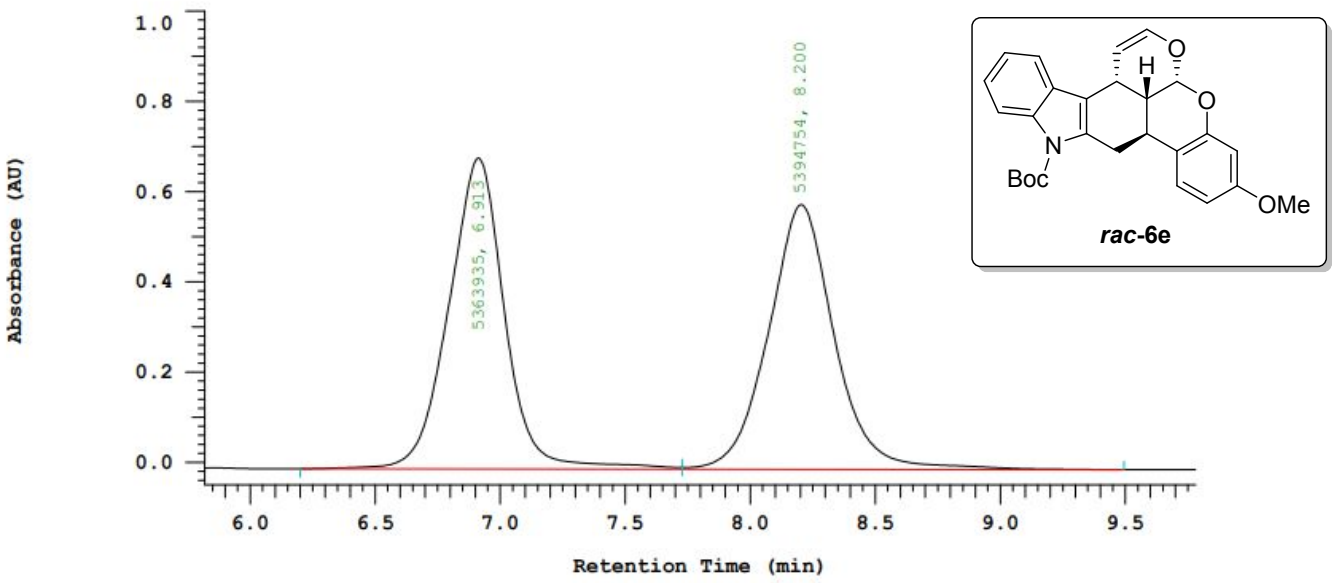

Chrom Type: Fixed WL Chromatogram, 205 nm

Peak Quantitation: AREA

Calculation Method: AREAs

\begin{tabular}{ccccc} 
No. & RT & Area & Area & BC \\
\hline 1 & 6.913 & 5363935 & 49.857 & BV \\
2 & 8.200 & 5394754 & 50.143 & VB \\
\hline & & 10758689 & 100.000 & \\
\hline
\end{tabular}

\section{The HPLC of chiral $6 \mathrm{e}$}

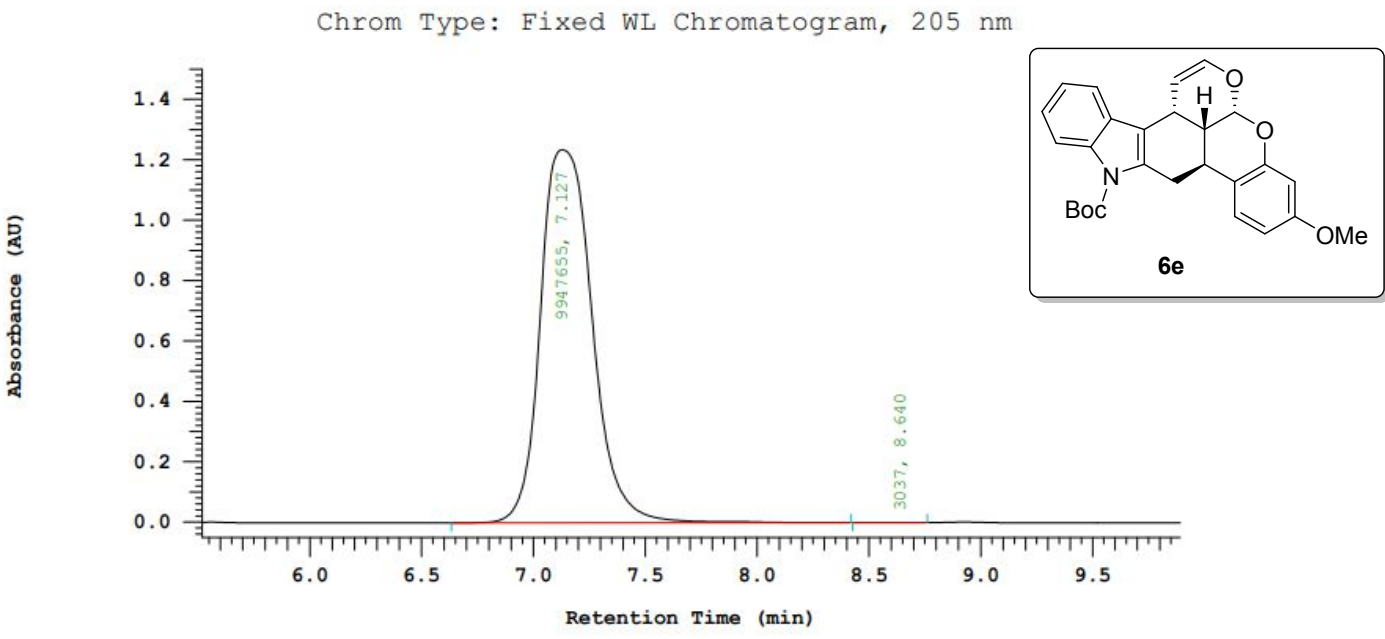

Chrom Type: Fixed WL Chromatogram, 205 nm

Peak Quantitation: AREA

Calculation Method: AREA

\begin{tabular}{rrrrr} 
No. & RT & Area & Area & BC \\
\hline 1 & 7.127 & 9947655 & 99.969 & BB \\
2 & 8.640 & 3037 & 0.031 & BB \\
\hline & & 9950692 & 100.000 & \\
\hline
\end{tabular}


The ${ }^{1} \mathrm{H}$ NMR spectrum of $6 \mathrm{f}\left(400 \mathrm{MHz}, \mathrm{CDCl}_{3}\right)$

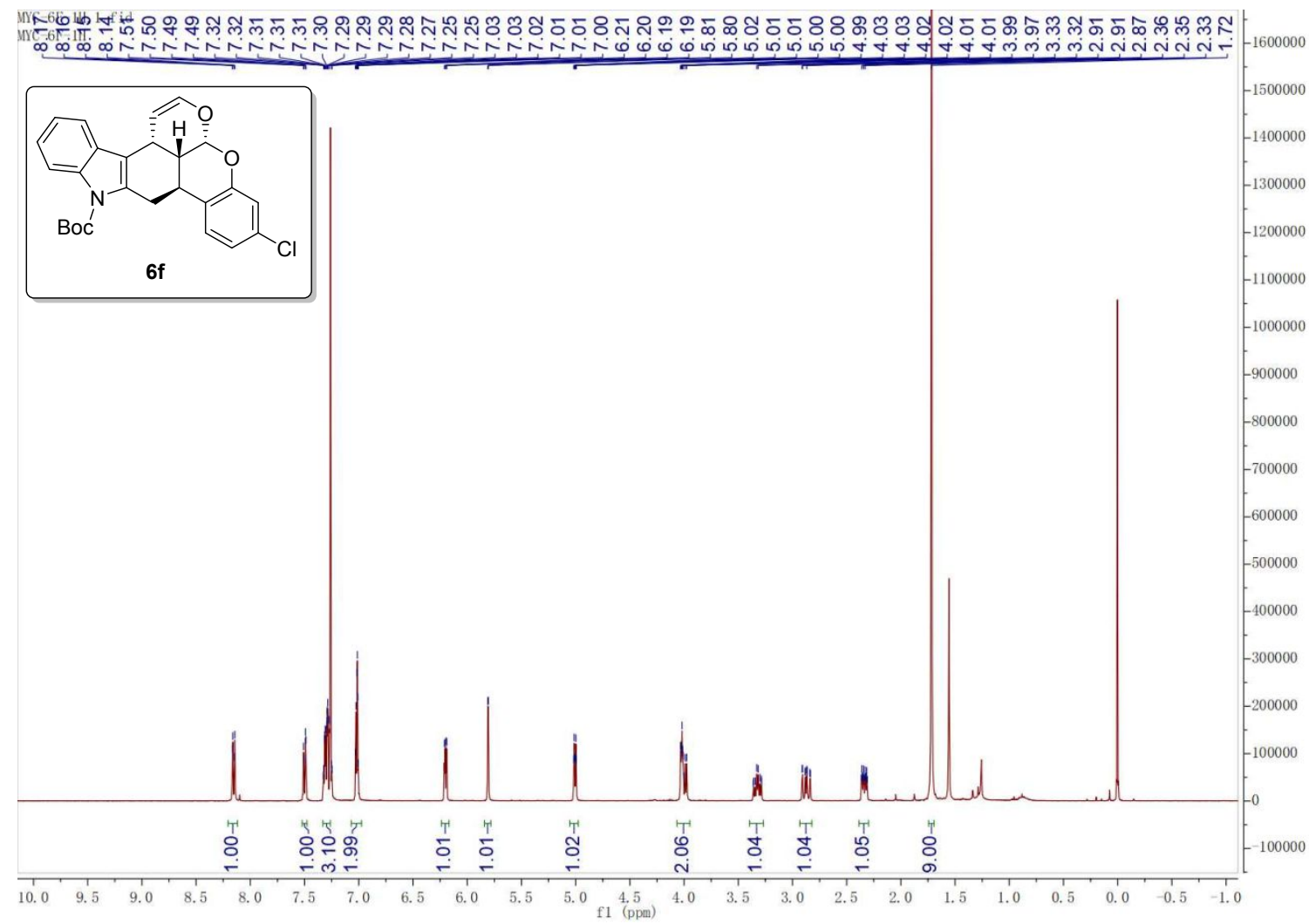

The ${ }^{13} \mathrm{C}$ NMR spectrum of $6 \mathrm{f}\left(100 \mathrm{MHz}, \mathrm{CDCl}_{3}\right)$
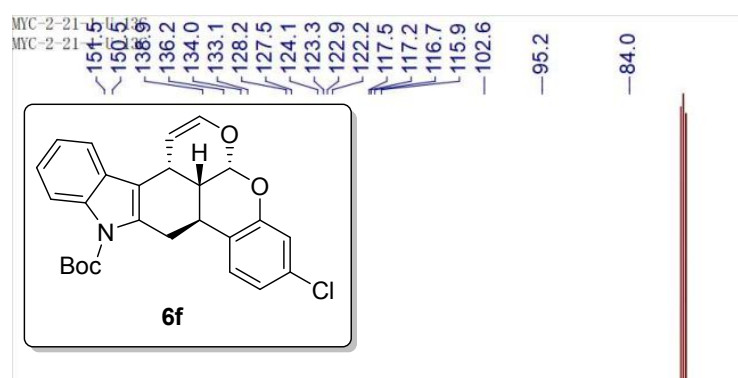

(


The HPLC of racemic $6 f$

Chrom Type: Fixed WL Chromatogram, 220 nm

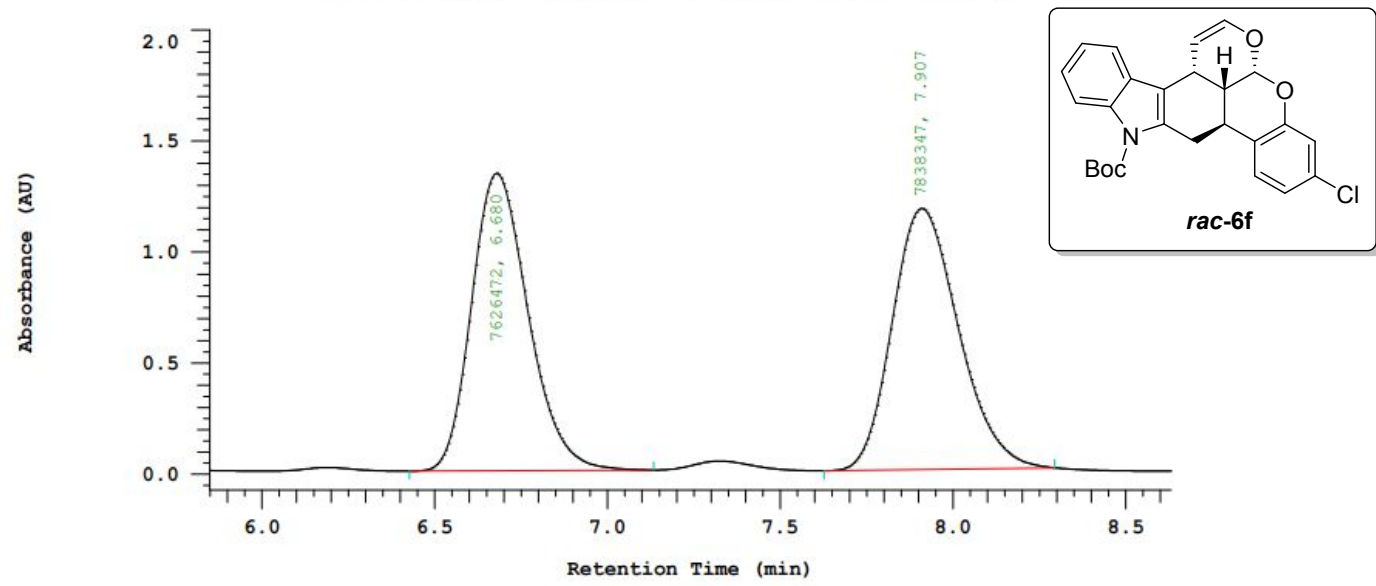

Chrom Type: Fixed WL Chromatogram, $220 \mathrm{~nm}$ Peak Quantitation: AREA

Calculation Method: AREA용

\begin{tabular}{ccccc} 
No. & RT & Area & Area o & BC \\
\hline 1 & 6.680 & 7626472 & 49.315 & BB \\
2 & 7.907 & 7838347 & 50.685 & BB \\
\hline & & 15464819 & 100.000 & \\
\hline
\end{tabular}

\section{The HPLC of chiral $6 f$}

Chrom Type: Fixed WL Chromatogram, $220 \mathrm{~nm}$

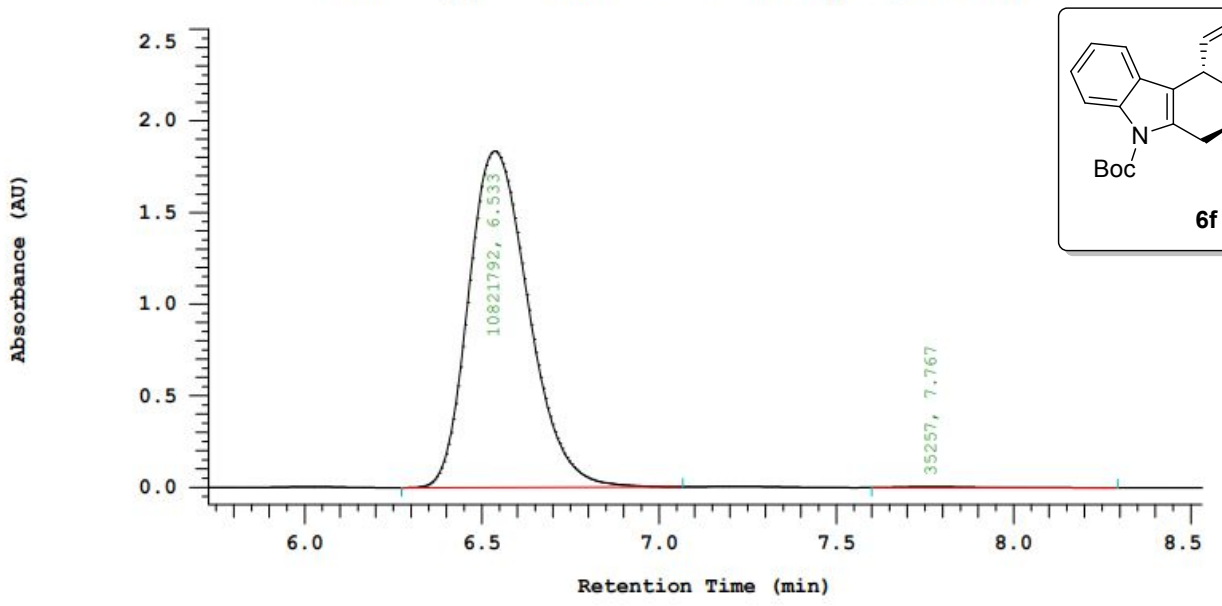

Chrom Type: Fixed WL Chromatogram, $220 \mathrm{~nm}$

Peak Quantitation: AREA

Calculation Method: AREA응

\begin{tabular}{rrrrr} 
No. & RT & \multicolumn{1}{c}{ Area } & Area & BC \\
\hline 1 & 6.533 & 10821792 & 99.675 & BB \\
2 & 7.767 & 35257 & 0.325 & BB \\
\hline & & 10857049 & 100.000 & \\
\hline
\end{tabular}


The ${ }^{1} \mathrm{H}$ NMR spectrum of $6 \mathrm{~g}\left(400 \mathrm{MHz}, \mathrm{CDCl}_{3}\right)$

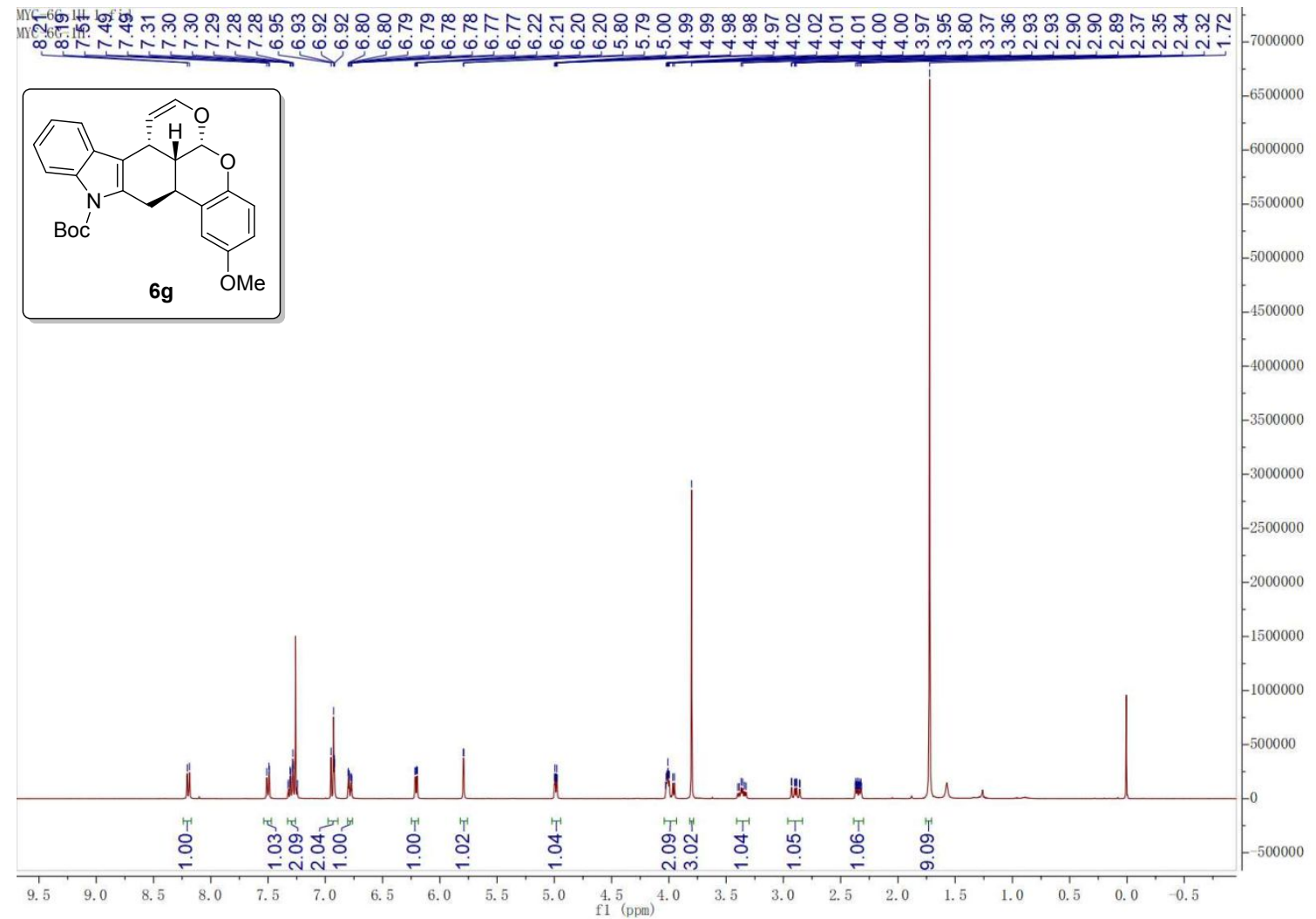

The ${ }^{13} \mathrm{C}$ NMR spectrum of $6 \mathrm{~g}\left(100 \mathrm{MHz}, \mathrm{CDCl}_{3}\right)$
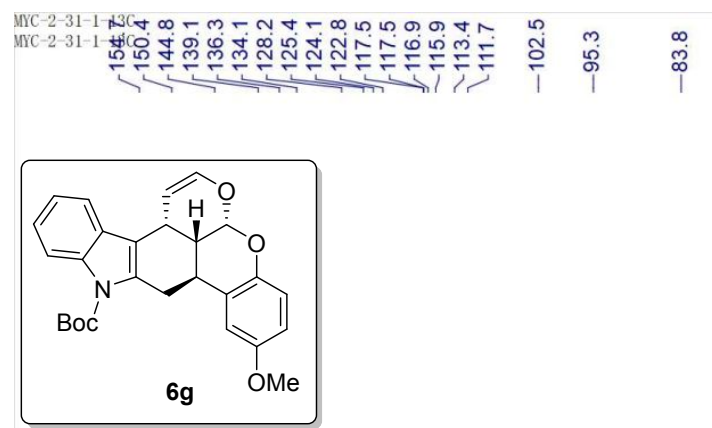


\section{The HPLC of racemic $6 \mathrm{~g}$}

Chrom Type: Fixed WL Chromatogram, 205 nm

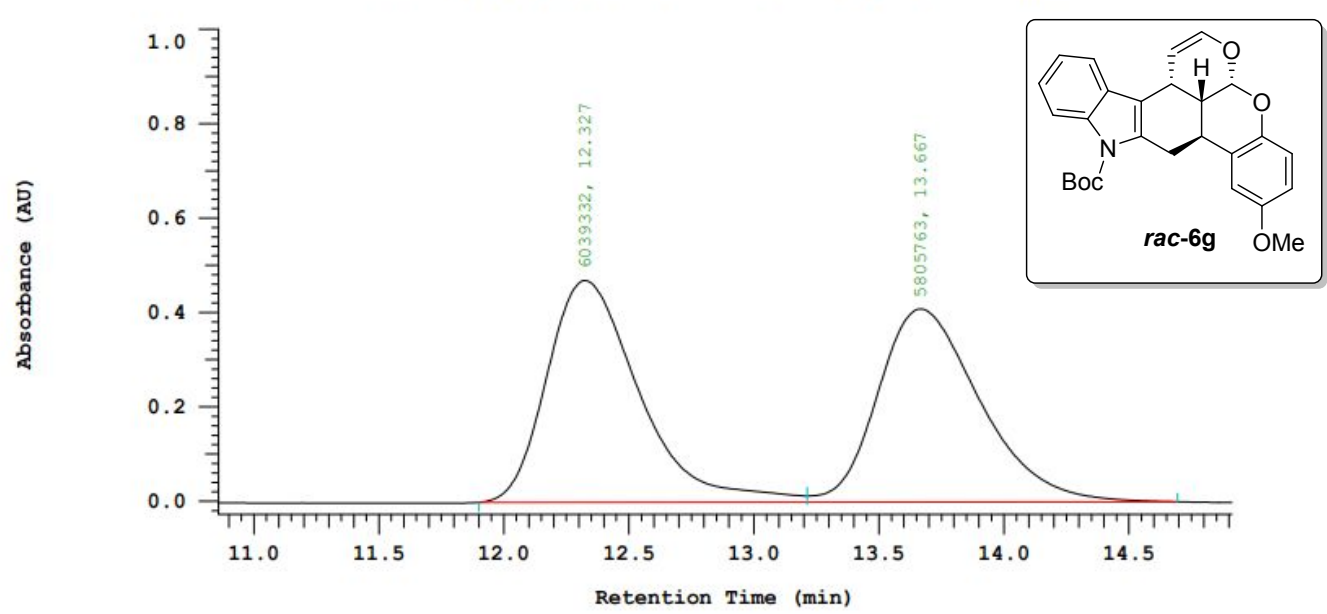

Chrom Type: Fixed WL Chromatogram, 205 nm

Peak Quantitation: AREA

Calculation Method: AREA

\begin{tabular}{ccccc} 
No. & RT & Area & Area & BC \\
\hline 1 & 12.327 & 6039332 & 50.986 & BV \\
2 & 13.667 & 5805763 & 49.014 & VB \\
\hline & & 11845095 & 100.000 & \\
\hline
\end{tabular}

\section{The HPLC of chiral $6 \mathrm{~g}$}

Chrom Type: Fixed WL Chromatogram, 205 nm

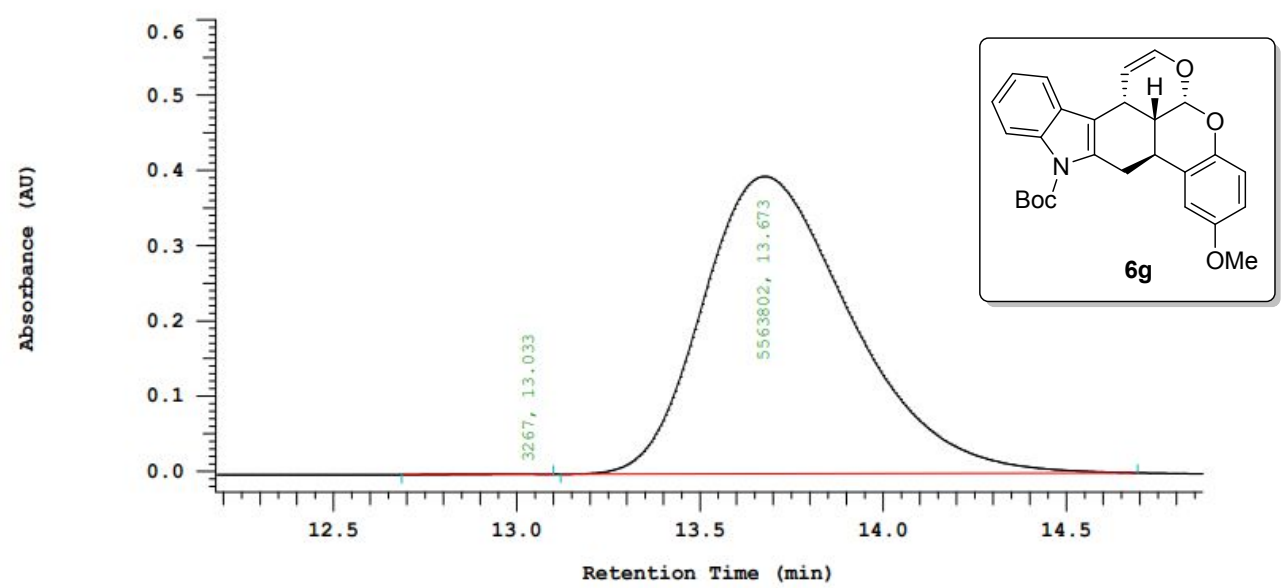

Chrom Type: Fixed WL Chromatogram, 205 nm

Peak Quantitation: AREA

Calculation Method: AREAs

\begin{tabular}{crrrr} 
No. & RT & Area & Area & BC \\
\hline 1 & 13.033 & 3267 & 0.059 & BB \\
2 & 13.673 & 5563802 & 99.941 & BB \\
\hline & & 5567069 & 100.000 & \\
\hline
\end{tabular}


The ${ }^{1} \mathrm{H}$ NMR spectrum of $6 \mathrm{~h}\left(400 \mathrm{MHz}, \mathrm{CDCl}_{3}\right)$

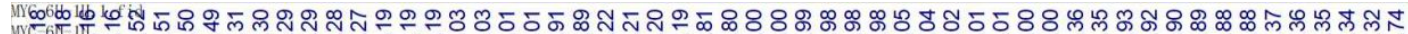
0000 N
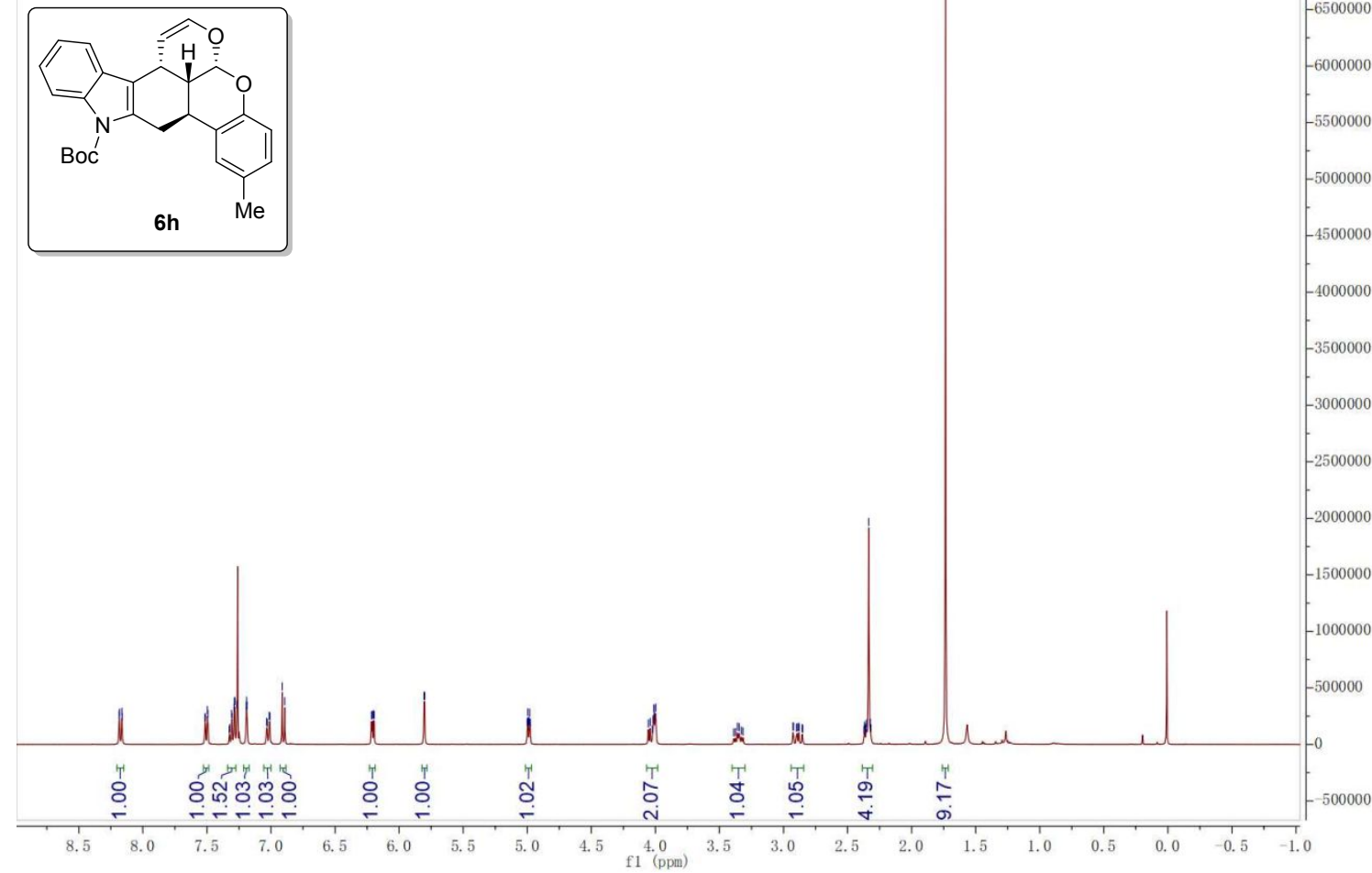

The ${ }^{13} \mathrm{C}$ NMR spectrum of $6 \mathrm{~h}\left(100 \mathrm{MHz}, \mathrm{CDCl}_{3}\right)$

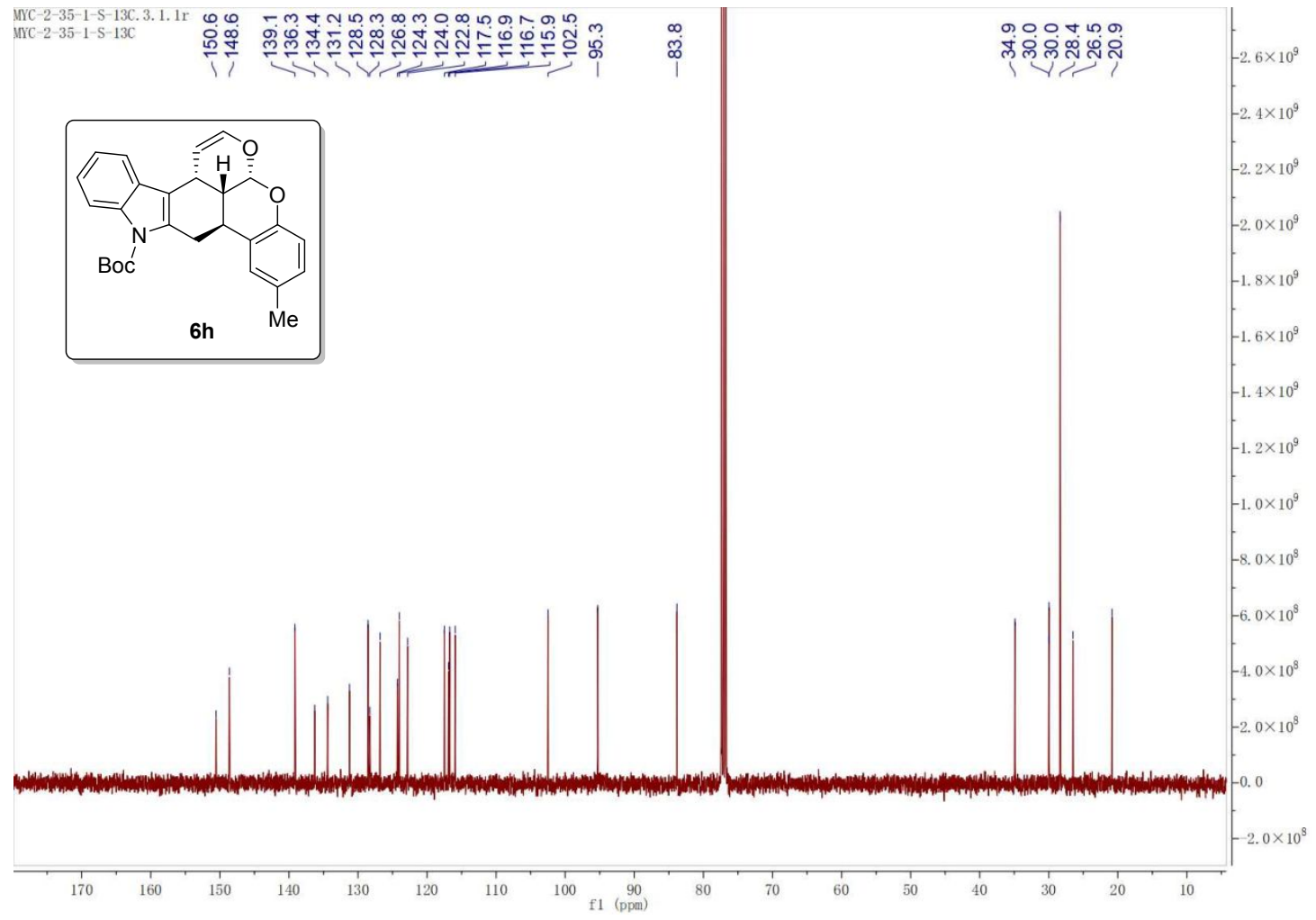




\section{The HPLC of racemic $6 \mathrm{~h}$}

Chrom Type: Fixed WL Chromatogram, 205 nm

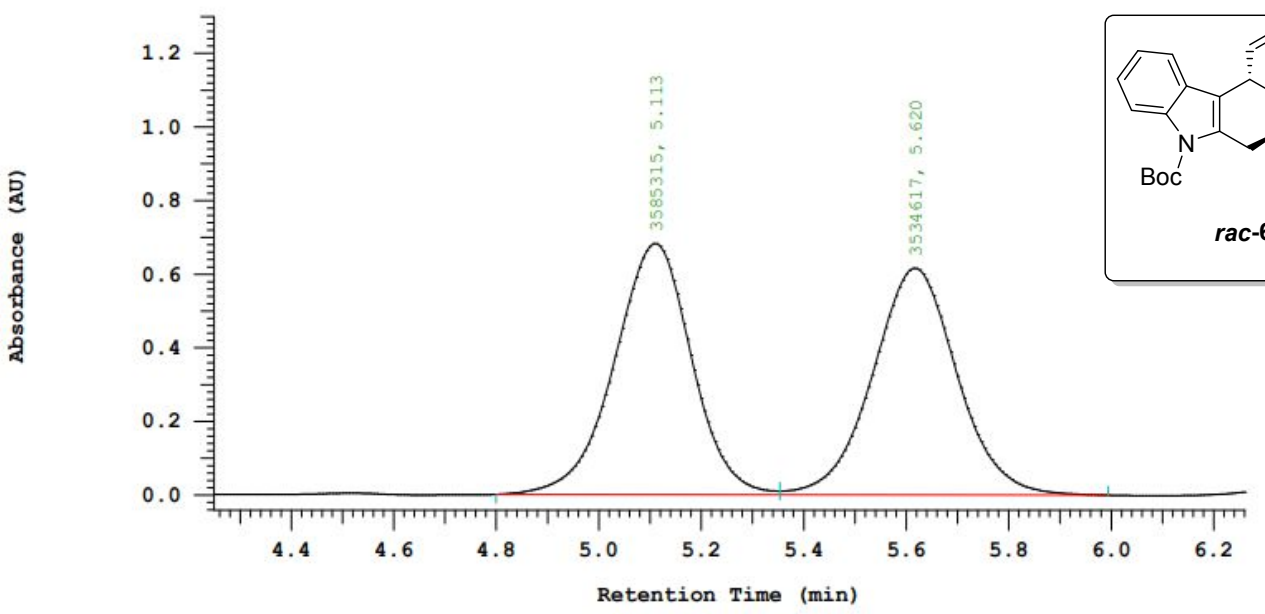

Chrom Type: Fixed WL Chromatogram, $205 \mathrm{~nm}$

Peak Quantitation: AREA

Calculation Method: AREA\%

\begin{tabular}{ccccc} 
No. & RT & Area & Area & BC \\
\hline 1 & 5.113 & 3585315 & 50.356 & BV \\
2 & 5.620 & 3534617 & 49.644 & VB \\
\hline & 7119932 & 100.000 & \\
\hline
\end{tabular}

\section{The HPLC of chiral $6 \mathrm{~h}$}

Chrom Type: Fixed WL Chromatogram, 205 nm
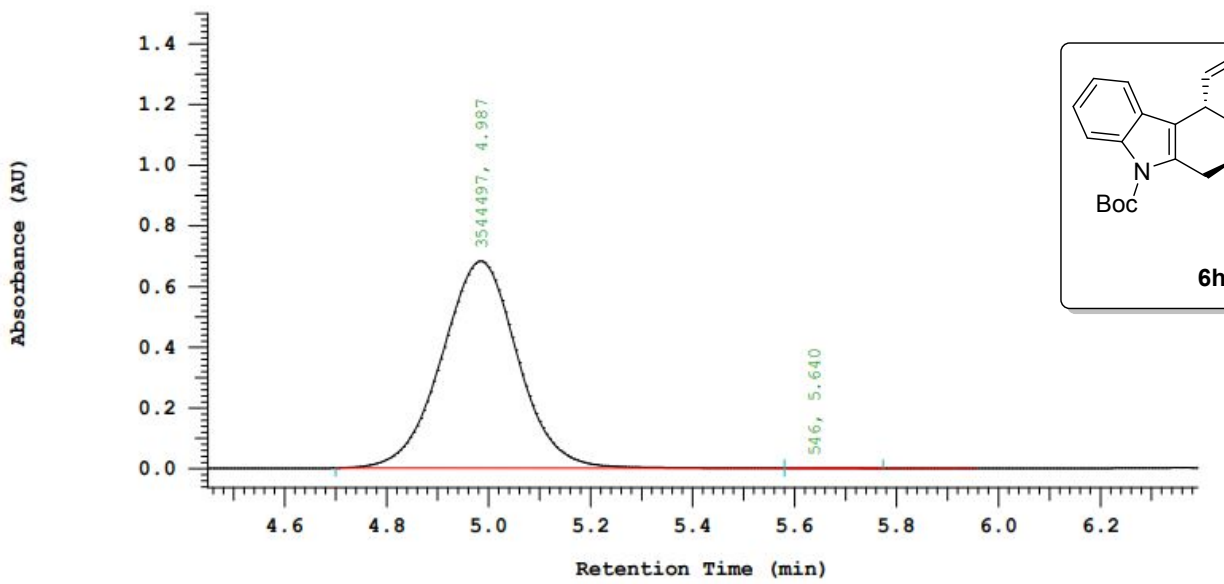

Chrom Type: Fixed WL Chromatogram, 205 nm

Peak Quantitation: AREA

Calculation Method: AREA용

\begin{tabular}{rrrrr} 
No. & RT & Area & Area & BC \\
\hline 1 & 4.987 & 3544497 & 99.985 & BV \\
2 & 5.640 & 546 & 0.015 & TBB \\
\hline & & 3545043 & 100.000 & \\
\hline
\end{tabular}


The ${ }^{1} \mathrm{H}$ NMR spectrum of $6 \mathrm{i}\left(400 \mathrm{MHz}, \mathrm{CDCl}_{3}\right)$

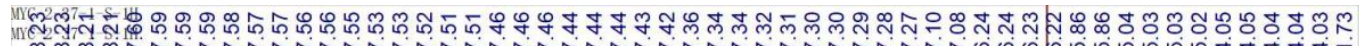

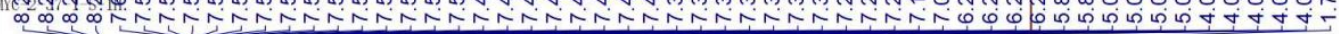
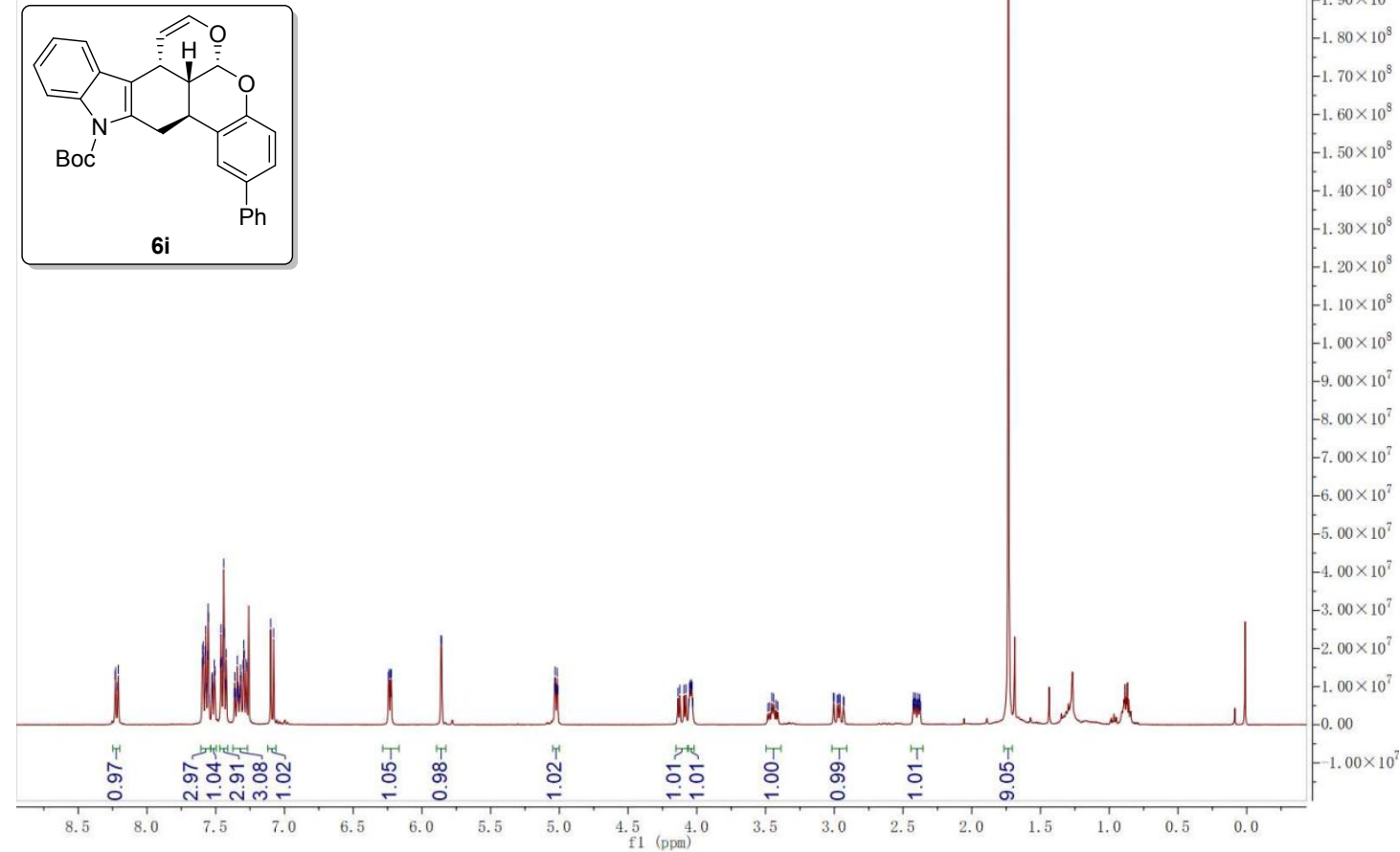

The ${ }^{13} \mathrm{C}$ NMR spectrum of $6 \mathrm{i}\left(100 \mathrm{MHz}, \mathrm{CDCl}_{3}\right)$

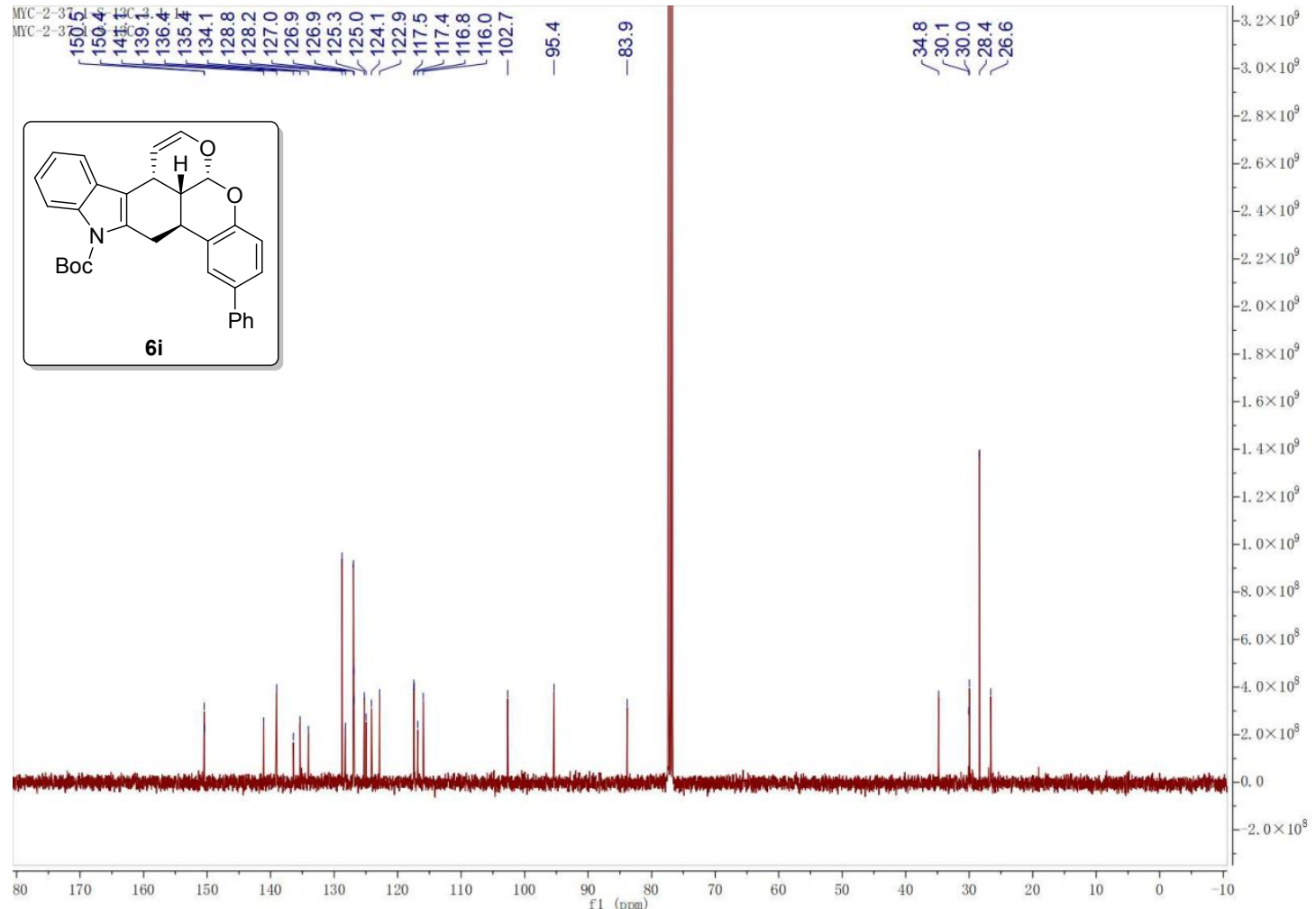




\section{The HPLC of racemic $6 i$}

Chrom Type: Fixed WL Chromatogram, 205 nm

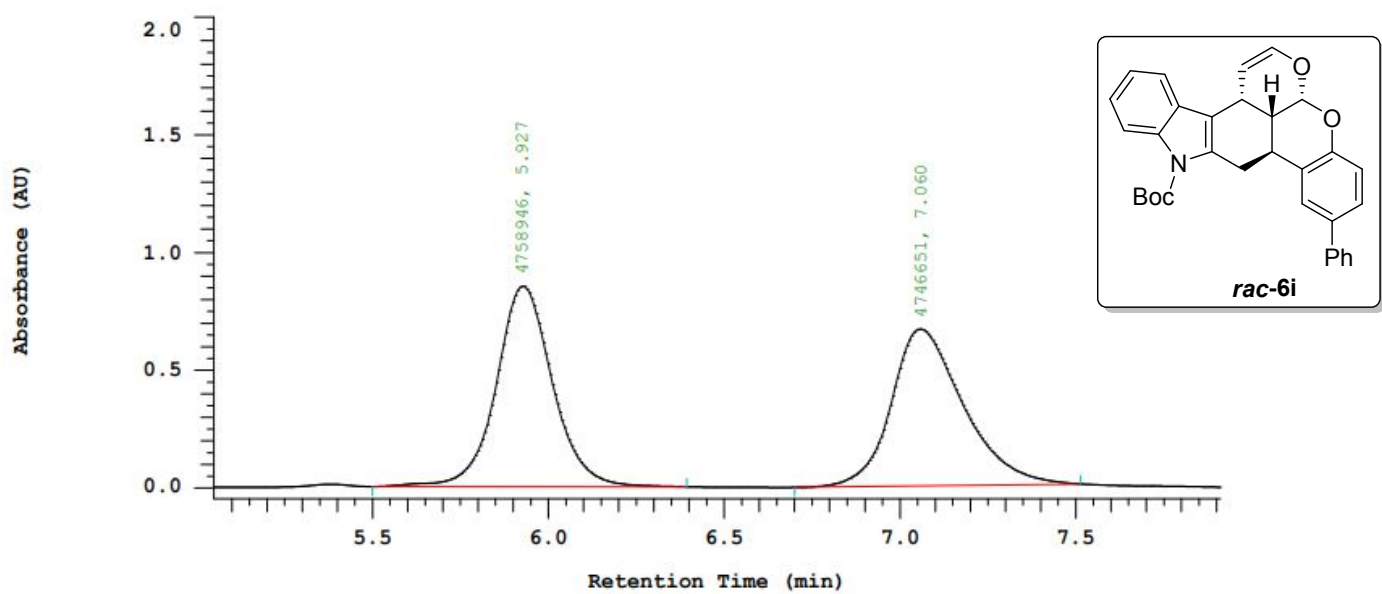

Chrom Type: Fixed WL Chromatogram, 205 nm Peak Quantitation: AREA

Calculation Method: AREA

\begin{tabular}{ccccc} 
No. & RT & Area & Area s & BC \\
\hline 1 & 5.927 & 4758946 & 50.065 & BB \\
2 & 7.060 & 4746651 & 49.935 & BB \\
\hline & & 9505597 & 100.000 & \\
\hline
\end{tabular}

\section{The HPLC of chiral $6 i$}

Chrom Type: Fixed WL Chromatogram, 205 nm

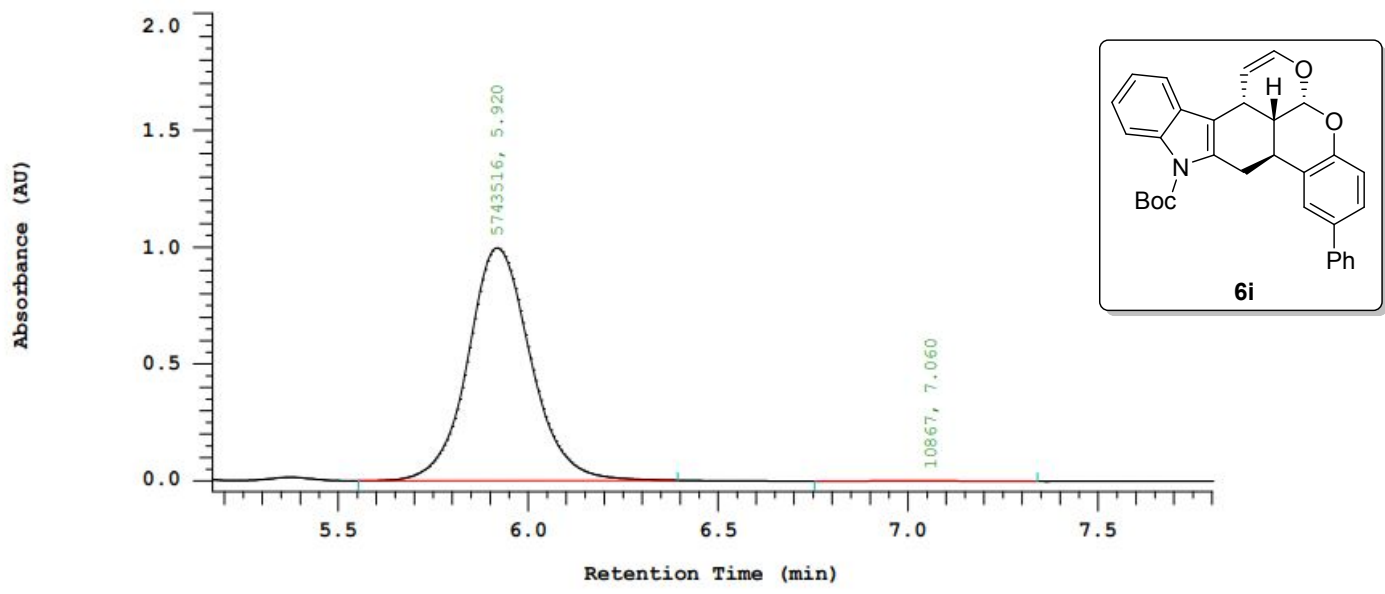

Chrom Type: Fixed WL Chromatogram, 205 nm

Peak Quantitation: AREA

Calculation Method: AREA\%

\begin{tabular}{crrrr} 
No. & RT & Area & Area & BC \\
\hline 1 & 5.920 & 5743516 & 99.811 & BB \\
2 & 7.060 & 10867 & 0.189 & BB \\
\hline & & 5754383 & 100.000 &
\end{tabular}


The ${ }^{1} \mathrm{H}$ NMR spectrum of $6 \mathrm{j}\left(400 \mathrm{MHz}, \mathrm{CDCl}_{3}\right)$

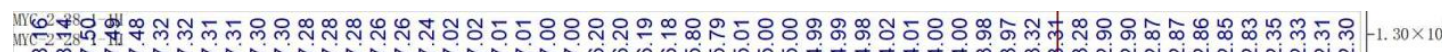

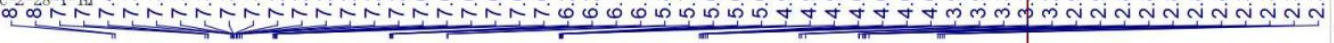
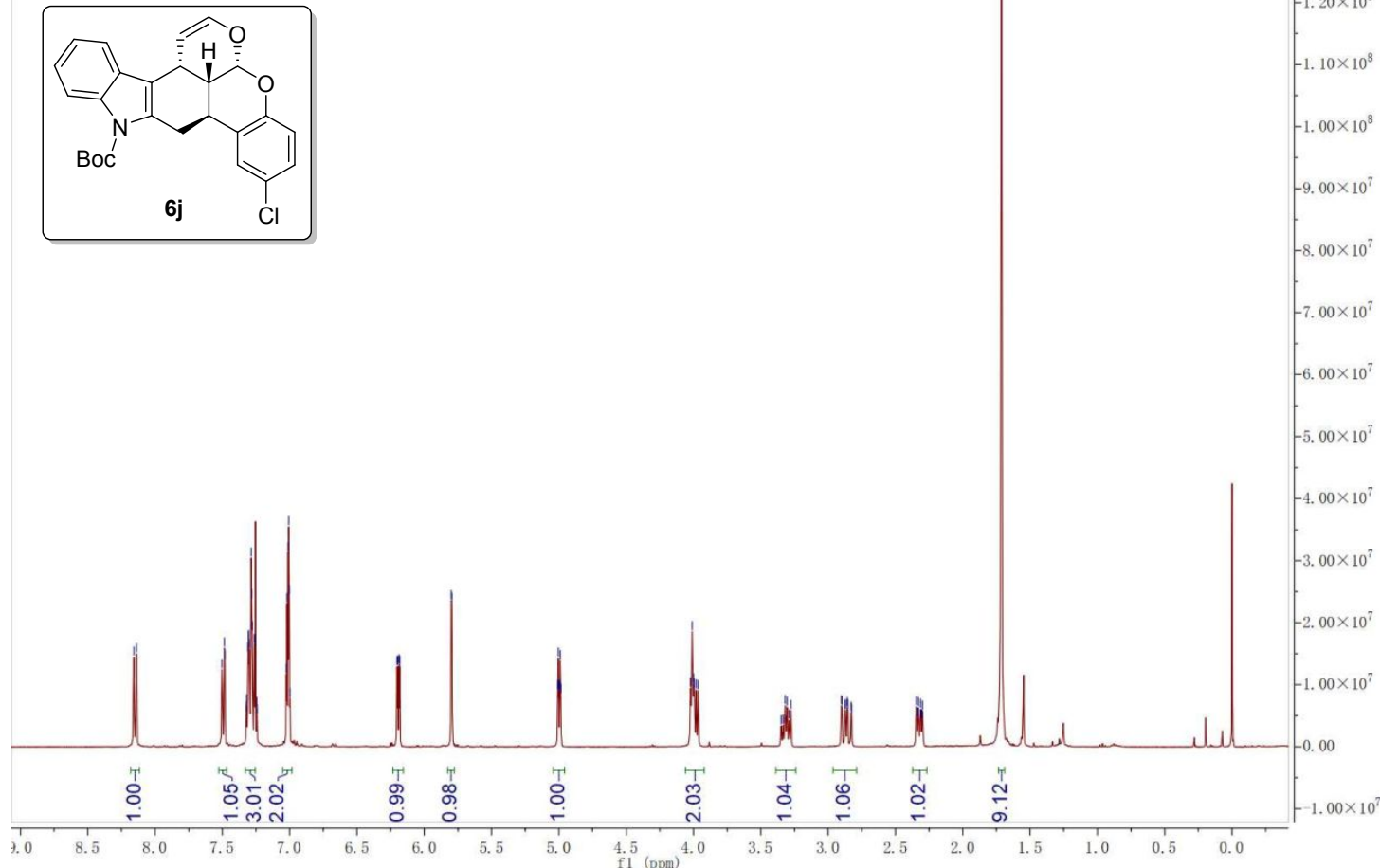

The ${ }^{13} \mathrm{C}$ NMR spectrum of $6 \mathrm{j}\left(100 \mathrm{MHz}, \mathrm{CDCl}_{3}\right)$

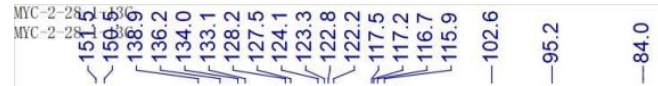

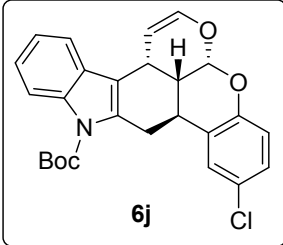

西

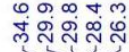

$5.0 \times 10^{8}$

4. $5 \times 10^{8}$

$-4.0 \times 10^{8}$

$-3.5 \times 10^{8}$

3. $0 \times 10^{8}$

$-2.5 \times 10^{9}$

$2.0 \times 10^{8}$

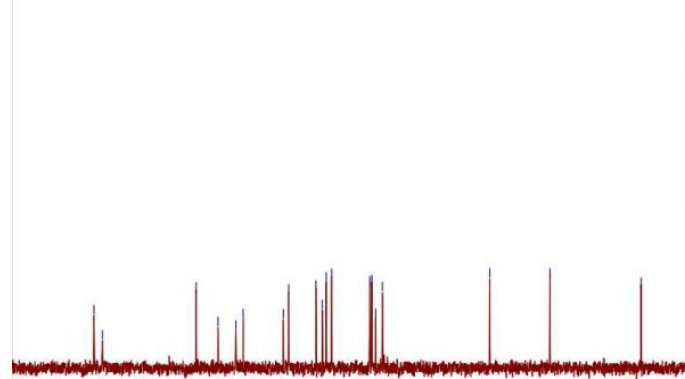

160

$140,130,120$

100

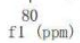




\section{The HPLC of racemic $6 j$}

Chrom Type: Fixed WL Chromatogram, 220 nm

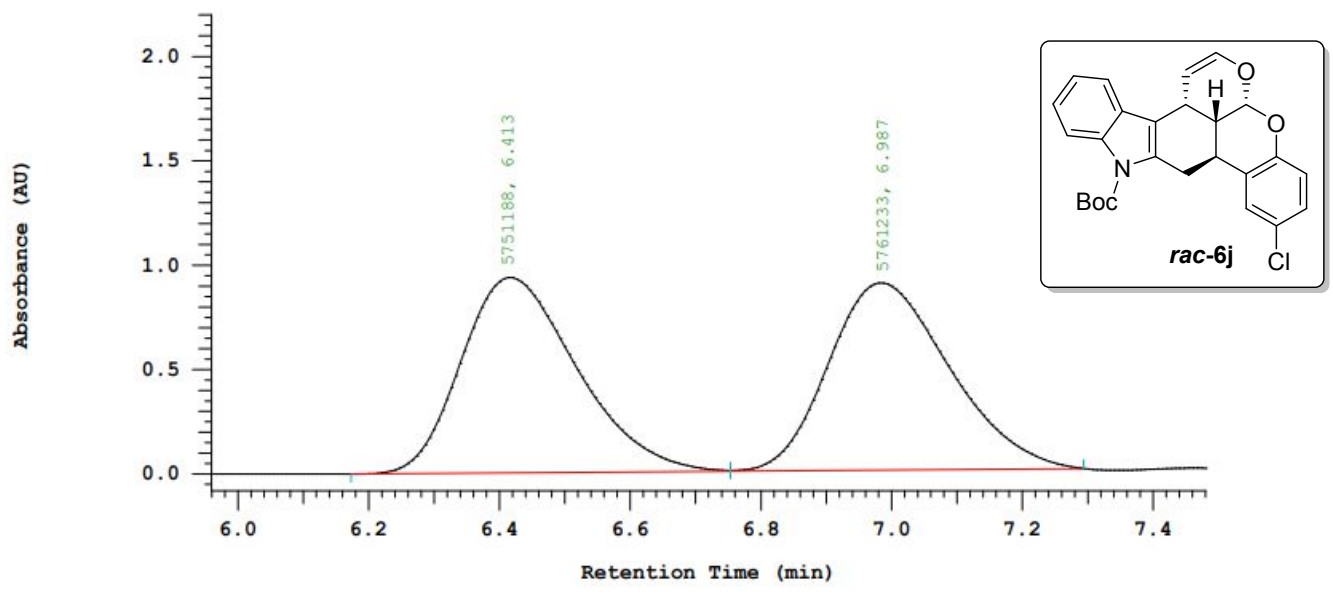

Chrom Type: Fixed WL Chromatogram, $220 \mathrm{~nm}$

Peak Quantitation: AREA

Calculation Method: AREA응

\begin{tabular}{ccccc} 
No. & RT & Area & Area & BC \\
\hline 1 & 6.413 & 5751188 & 49.956 & BV \\
2 & 6.987 & 5761233 & 50.044 & VB \\
\hline & & 11512421 & 100.000 & \\
\hline
\end{tabular}

\section{The HPLC of chiral 6j}

Chrom Type: Fixed WL Chromatogram, $220 \mathrm{~nm}$

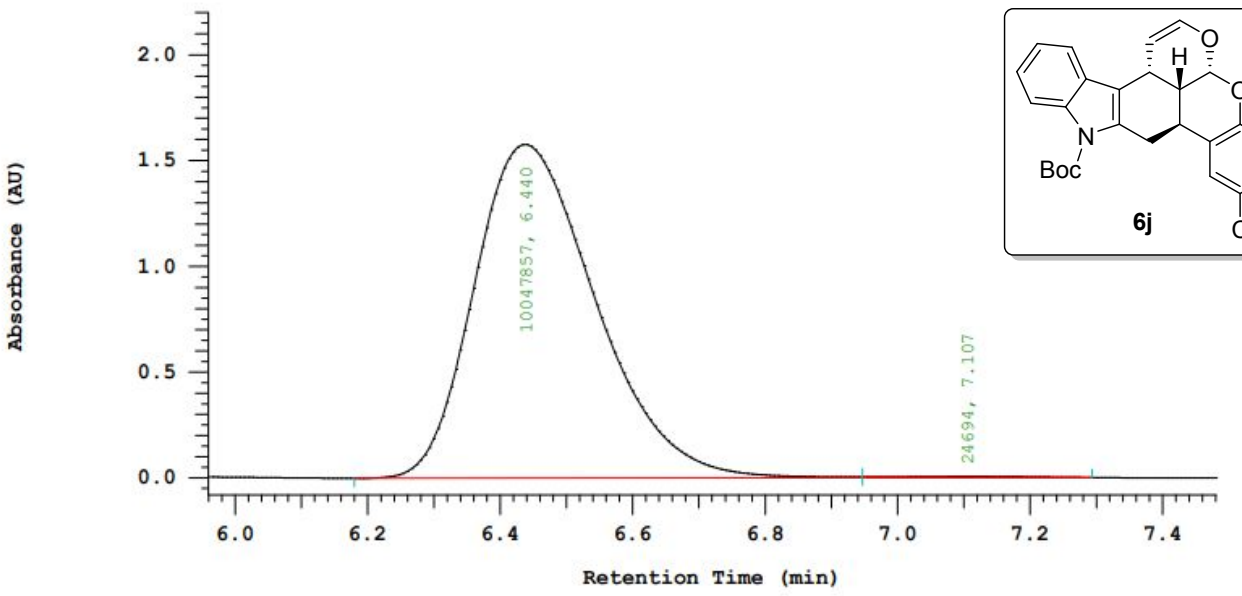

Chrom Type: Fixed WL Chromatogram, $220 \mathrm{~nm}$

Peak Quantitation: AREA

Calculation Method: AREA용

\begin{tabular}{rrrrr} 
No. & RT & \multicolumn{1}{c}{ Area } & Area $\%$ & BC \\
\hline 1 & 6.440 & 10047857 & 99.755 & BV \\
2 & 7.107 & 24694 & 0.245 & TBB \\
\hline & & 10072551 & 100.000 & \\
\hline
\end{tabular}


The ${ }^{1} \mathrm{H}$ NMR spectrum of $6 \mathrm{k}\left(400 \mathrm{MHz}, \mathrm{CDCl}_{3}\right)$

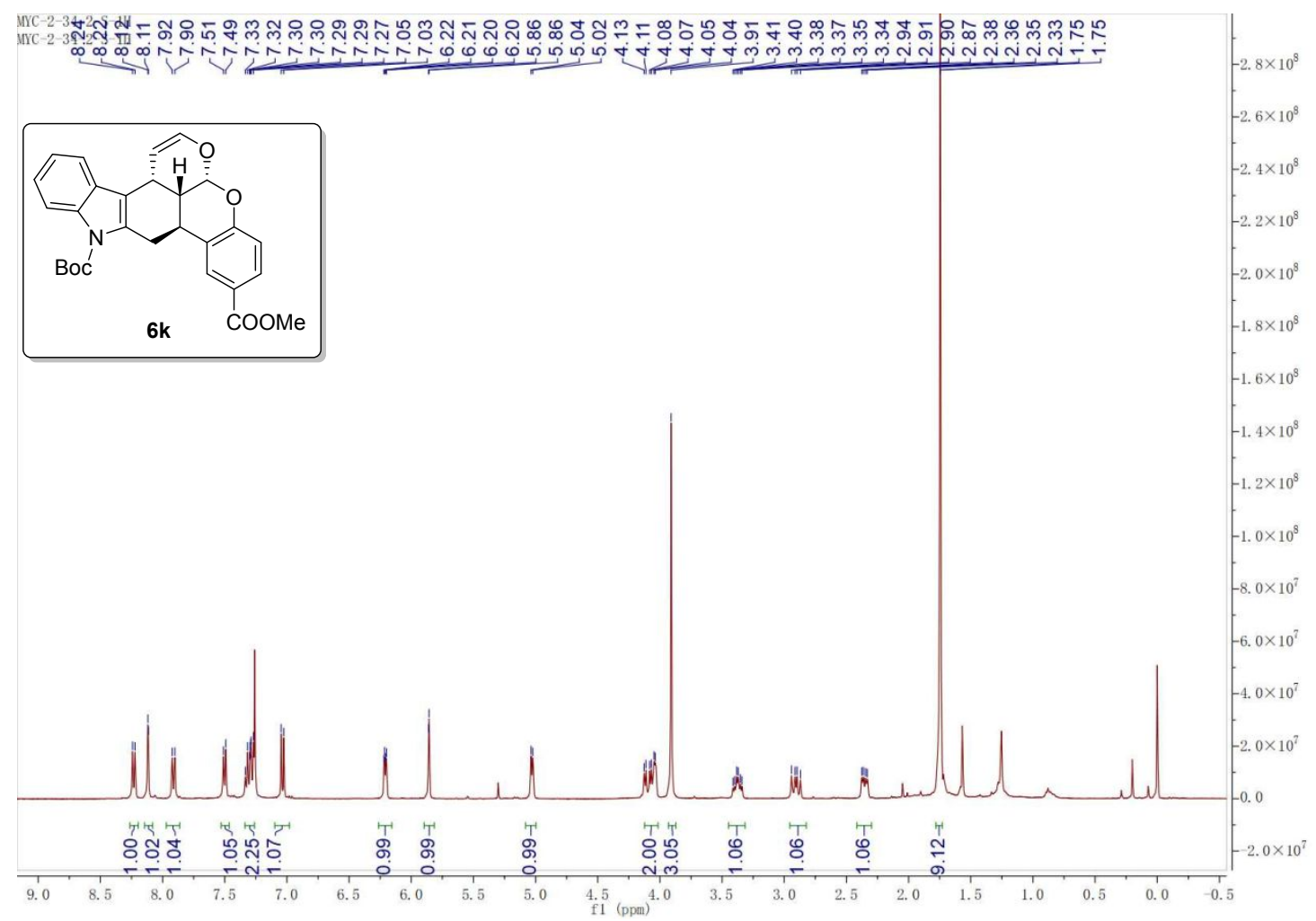

The ${ }^{13} \mathrm{C}$ NMR spectrum of $6 \mathrm{k}\left(100 \mathrm{MHz}, \mathrm{CDCl}_{3}\right)$

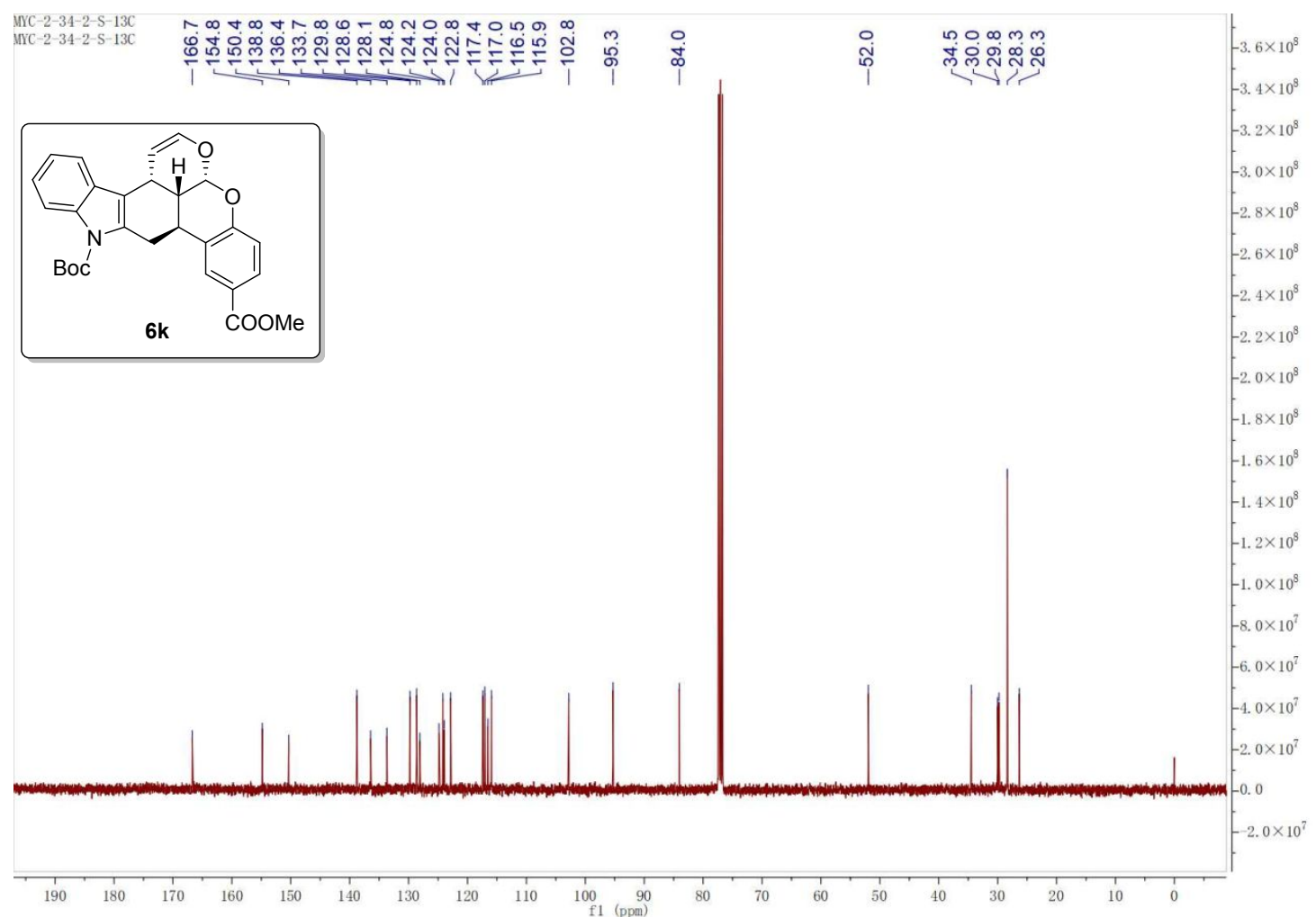




\section{The HPLC of racemic 6k}

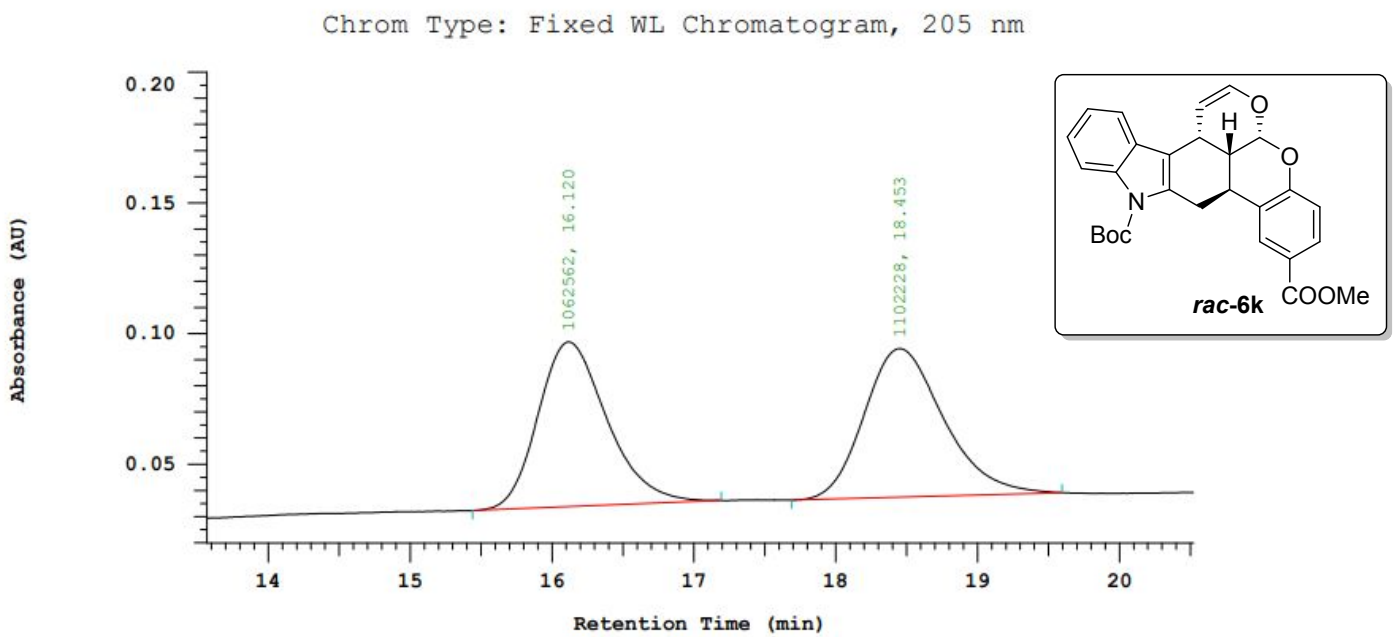

Chrom Type: Fixed WL Chromatogram, 205 nm Peak Quantitation: AREA

Calculation Method: AREA

\begin{tabular}{ccccc} 
No. & RT & Area & Area $\%$ & BC \\
\hline 1 & 16.120 & 1062562 & 49.084 & BB \\
2 & 18.453 & 1102228 & 50.916 & BB \\
\hline & & 2164790 & 100.000 & \\
\hline
\end{tabular}

\section{The HPLC of chiral 6k}

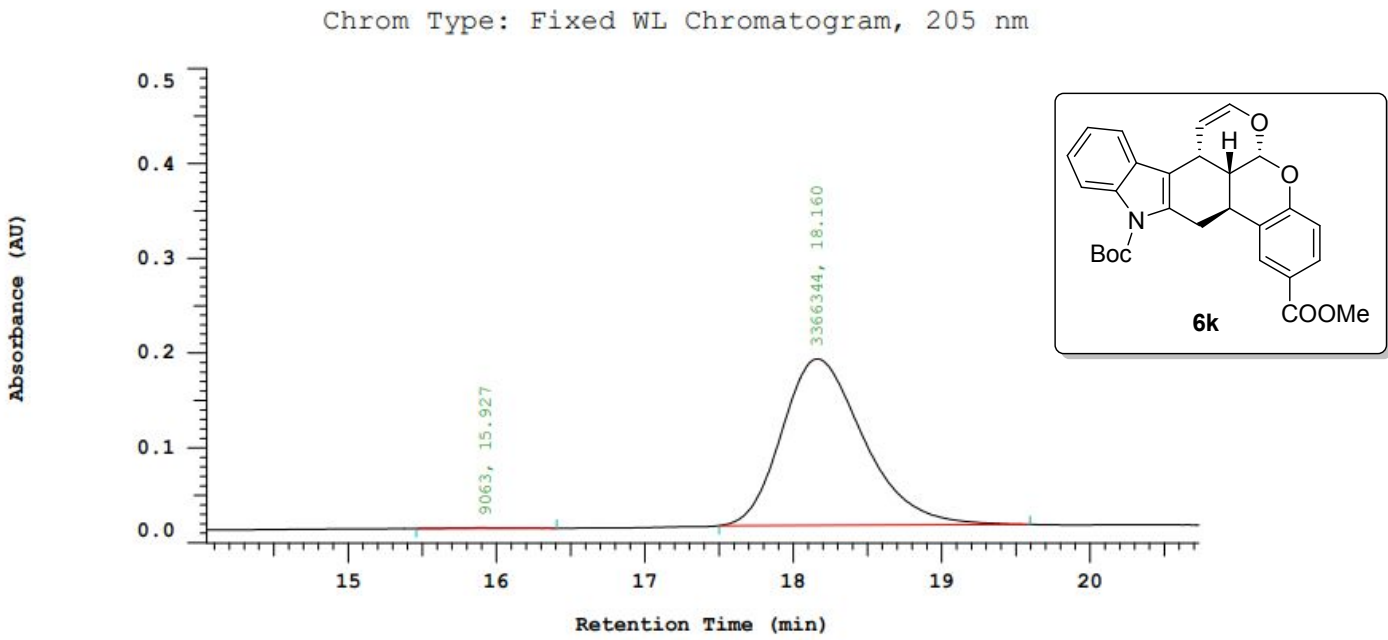

Chrom Type: Fixed WL Chromatogram, 205 nm Peak Quantitation: AREA

Calculation Method: AREA\%

\begin{tabular}{crrrr} 
No. & RT & Area & Area & BC \\
\hline 1 & 15.927 & 9063 & 0.269 & BB \\
2 & 18.160 & 3366344 & 99.731 & BB \\
\hline & & 3375407 & 100.000 & \\
\hline
\end{tabular}


The ${ }^{1} \mathrm{H}$ NMR spectrum of $61\left(400 \mathrm{MHz}, \mathrm{CDCl}_{3}\right)$

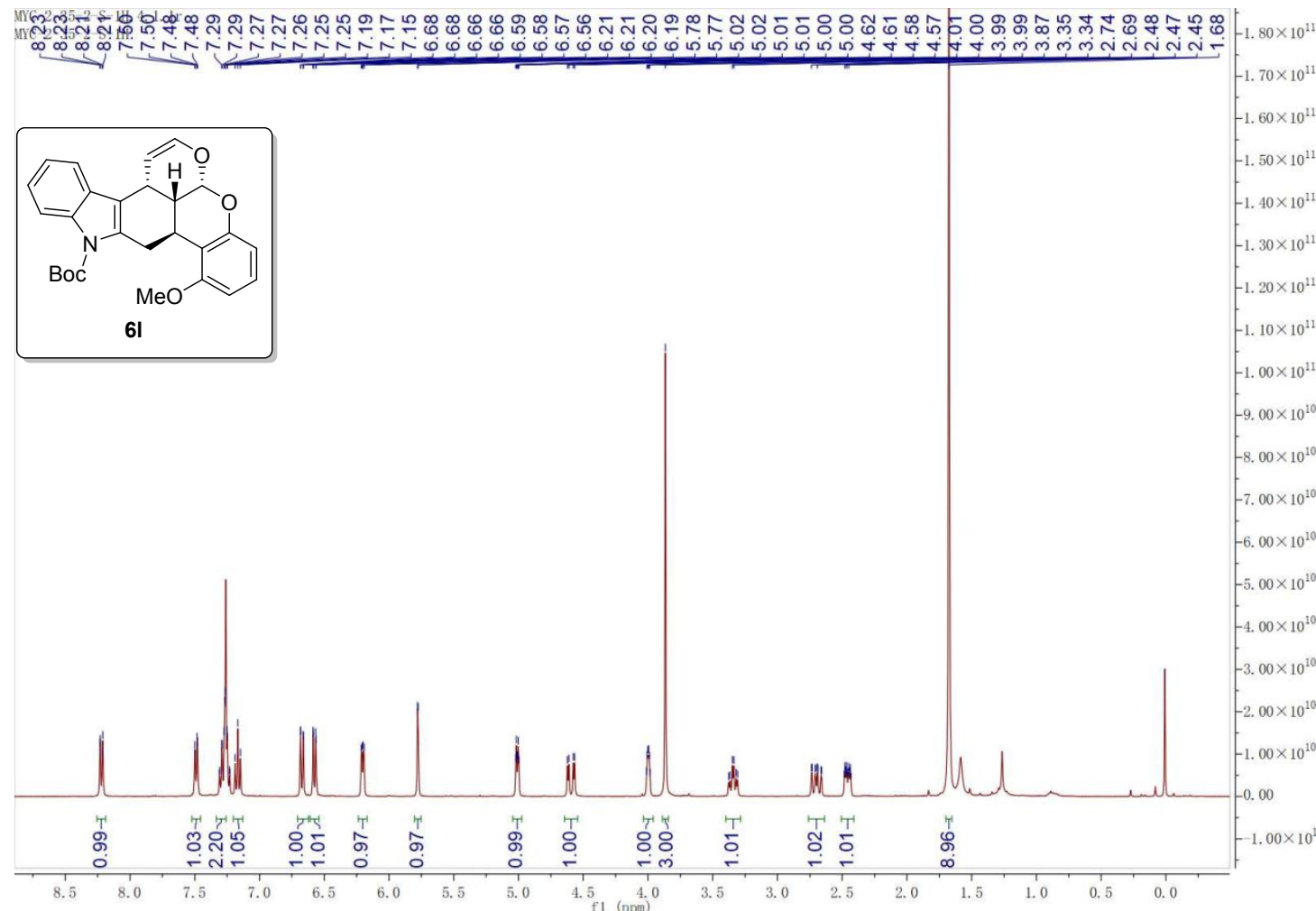

The ${ }^{13} \mathrm{C}$ NMR spectrum of $61\left(100 \mathrm{MHz}, \mathrm{CDCl}_{3}\right)$

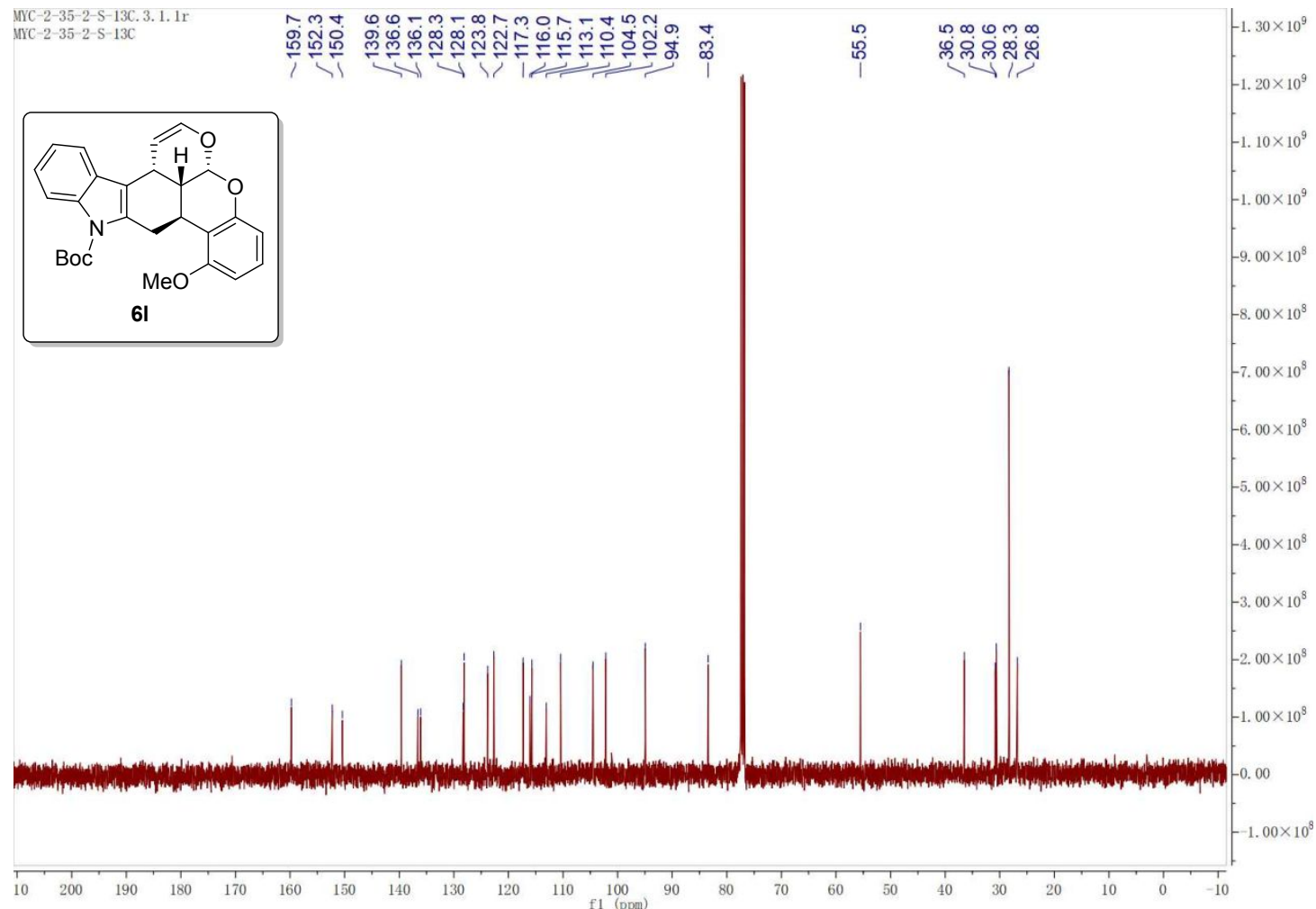


The HPLC of racemic 61

Chrom Type: Fixed WL Chromatogram, 205 nm

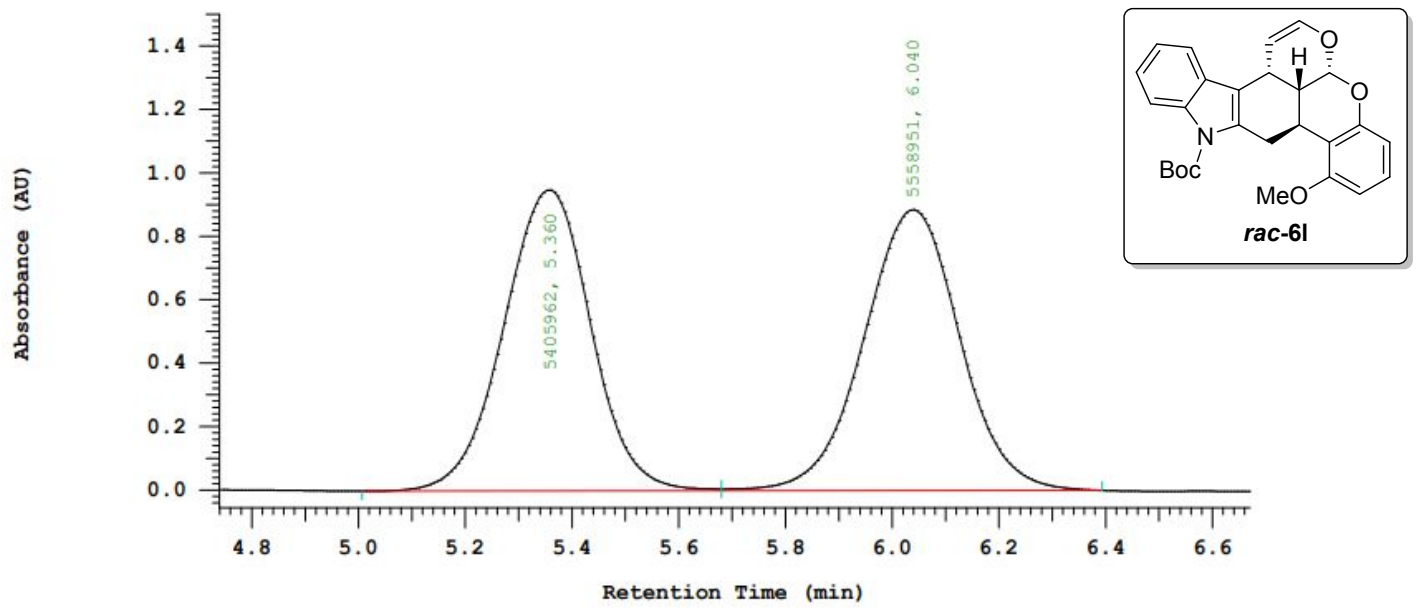

Chrom Type: Fixed WL Chromatogram, 205 nm Peak Quantitation: AREA

Calculation Method: AREA응

\begin{tabular}{ccccc} 
No. & RT & Area & Area & BC \\
\hline 1 & 5.360 & 5405962 & 49.302 & BV \\
2 & 6.040 & 5558951 & 50.698 & VB \\
\hline & & 10964913 & 100.000 & \\
\hline
\end{tabular}

\section{The HPLC of chiral 61}

Chrom Type: Fixed WL Chromatogram, 205 nm

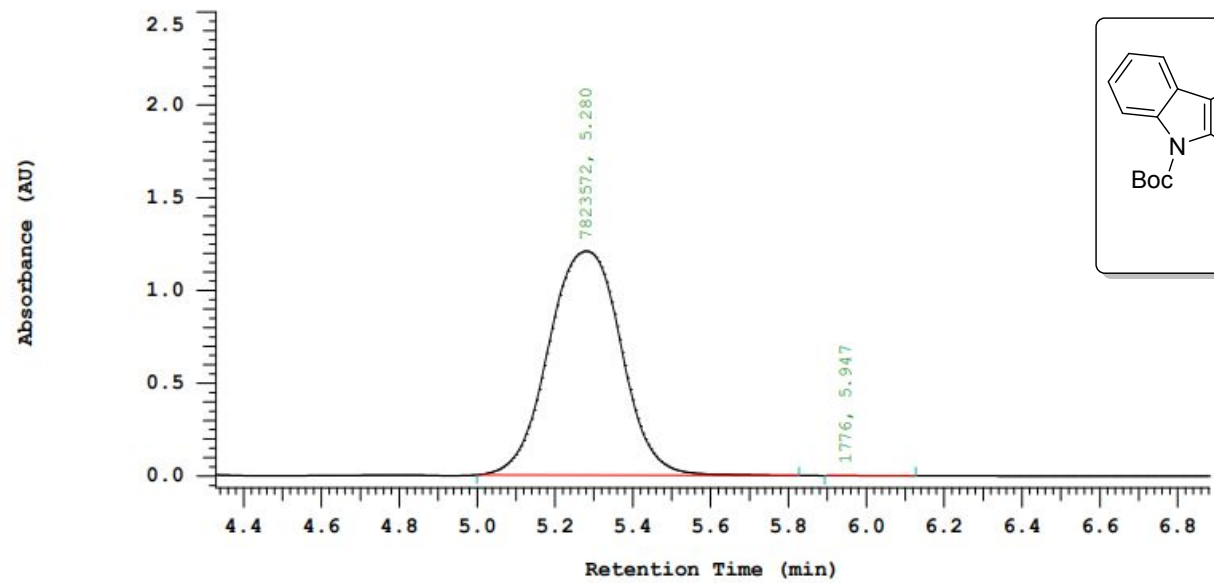

Chrom Type: Fixed WL Chromatogram, $205 \mathrm{~nm}$

Peak Quantitation: AREA

Calculation Method: AREA

\begin{tabular}{rrrrr} 
No. & RT & Area & Area & BC \\
\hline 1 & 5.280 & 7823572 & 99.977 & BB \\
2 & 5.947 & 1776 & 0.023 & BB \\
\hline & & 7825348 & 100.000 & \\
\hline
\end{tabular}


The ${ }^{1} \mathrm{H}$ NMR spectrum of $6 \mathrm{~m}\left(400 \mathrm{MHz}, \mathrm{CDCl}_{3}\right)$

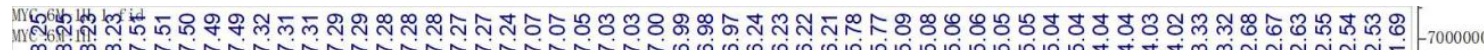

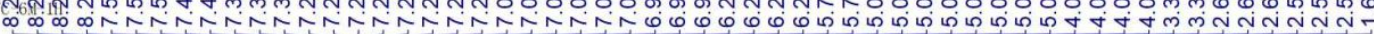
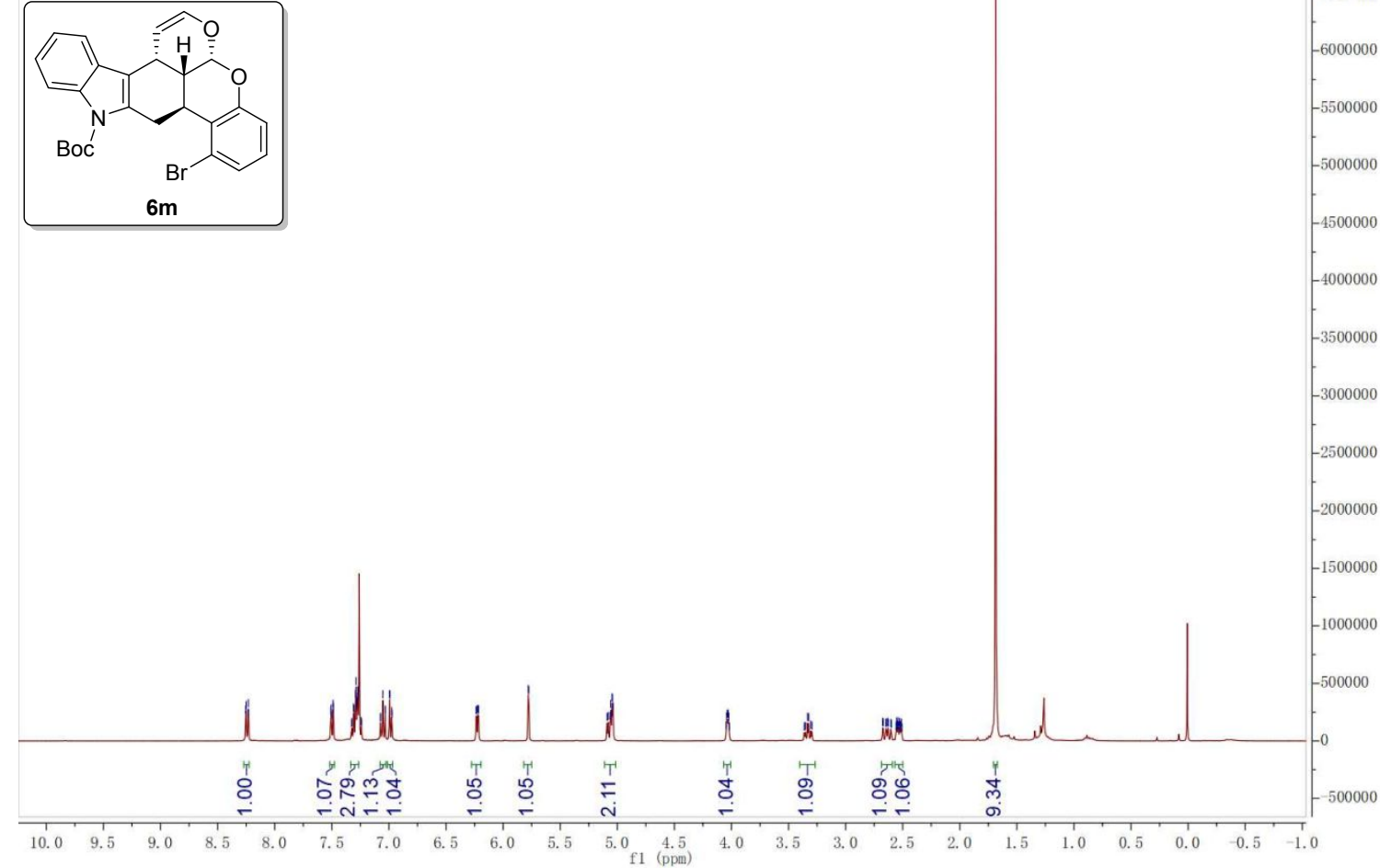

The ${ }^{13} \mathrm{C}$ NMR spectrum of $6 \mathrm{~m}\left(100 \mathrm{MHz}, \mathrm{CDCl}_{3}\right)$
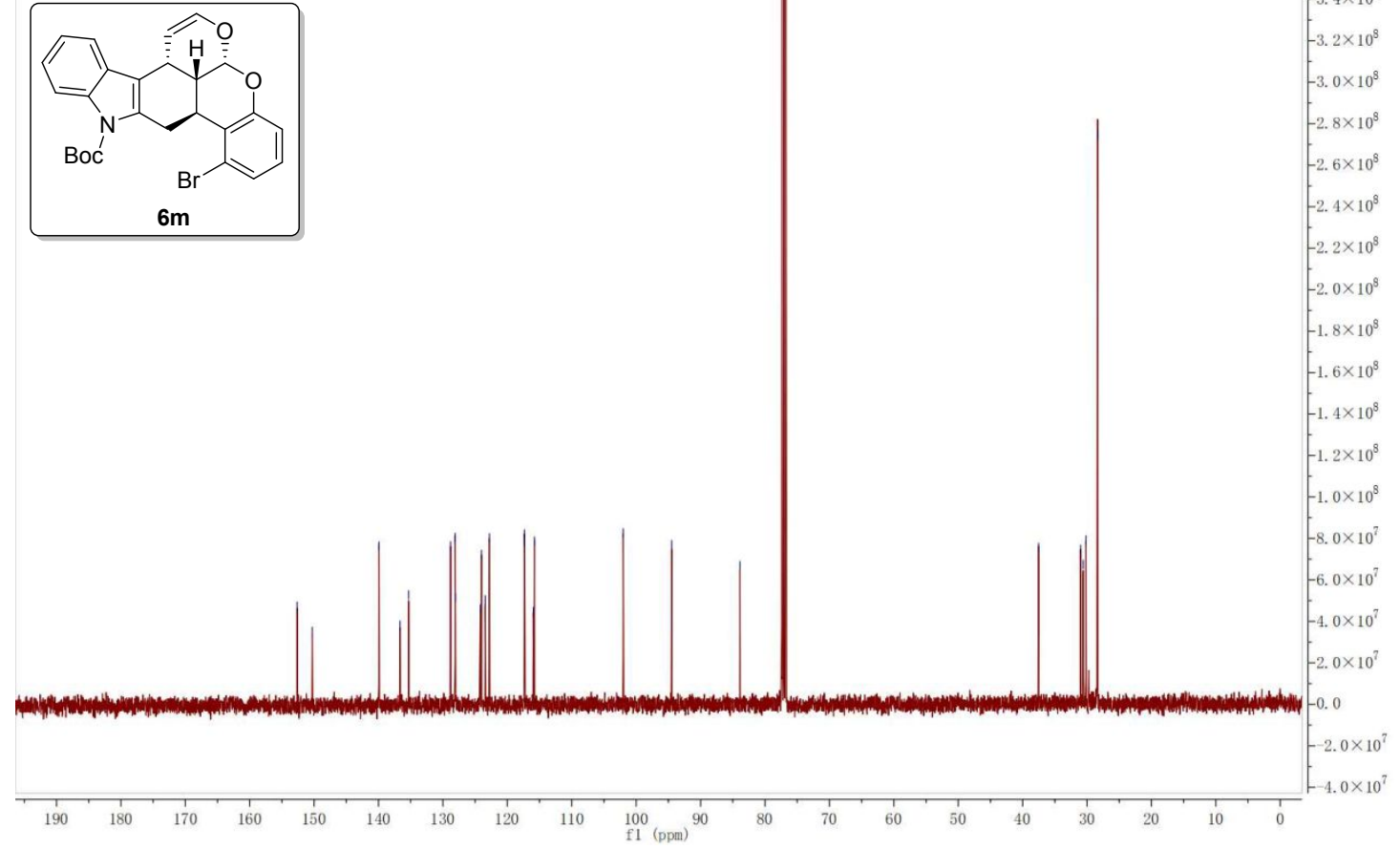


\section{The HPLC of racemic $6 \mathrm{~m}$}

Chrom Type: Fixed WL Chromatogram, 220 nm

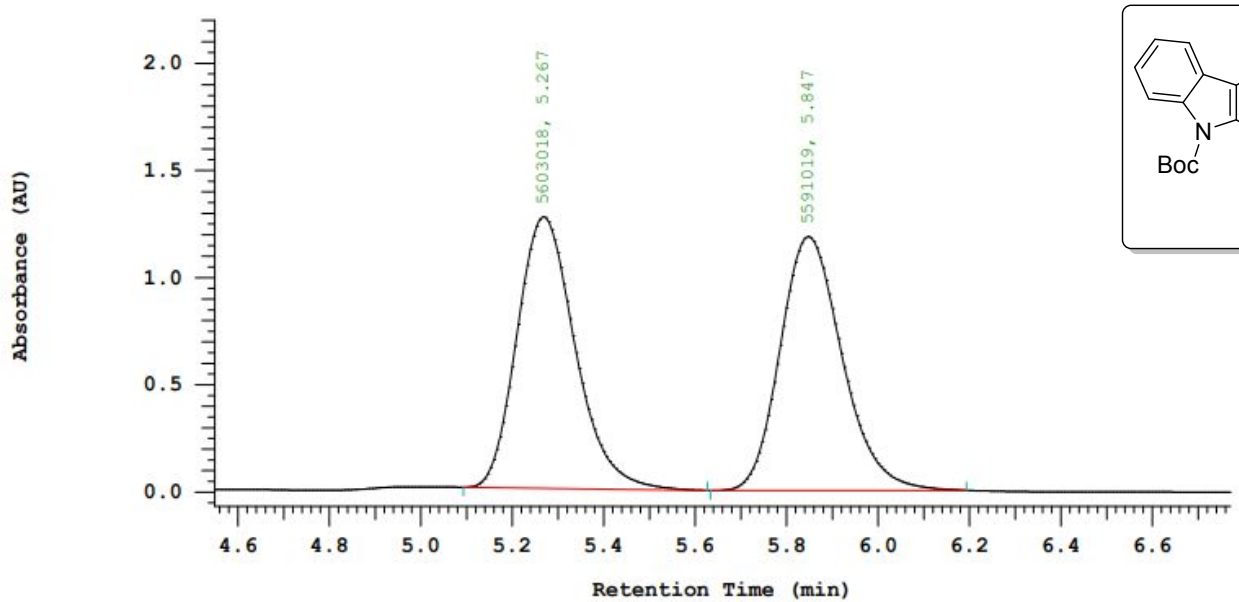

Chrom Type: Fixed WL Chromatogram, $220 \mathrm{~nm}$

Peak Quantitation: AREA

Calculation Method: AREA응

\begin{tabular}{ccccc} 
No. & RT & Area & Area 음 & BC \\
\hline 1 & 5.267 & 5603018 & 50.054 & BB \\
2 & 5.847 & 5591019 & 49.946 & BB \\
\hline & & 11194037 & 100.000
\end{tabular}

\section{The HPLC of chiral $6 \mathrm{~m}$}

Chrom Type: Fixed WL Chromatogram, $220 \mathrm{~nm}$

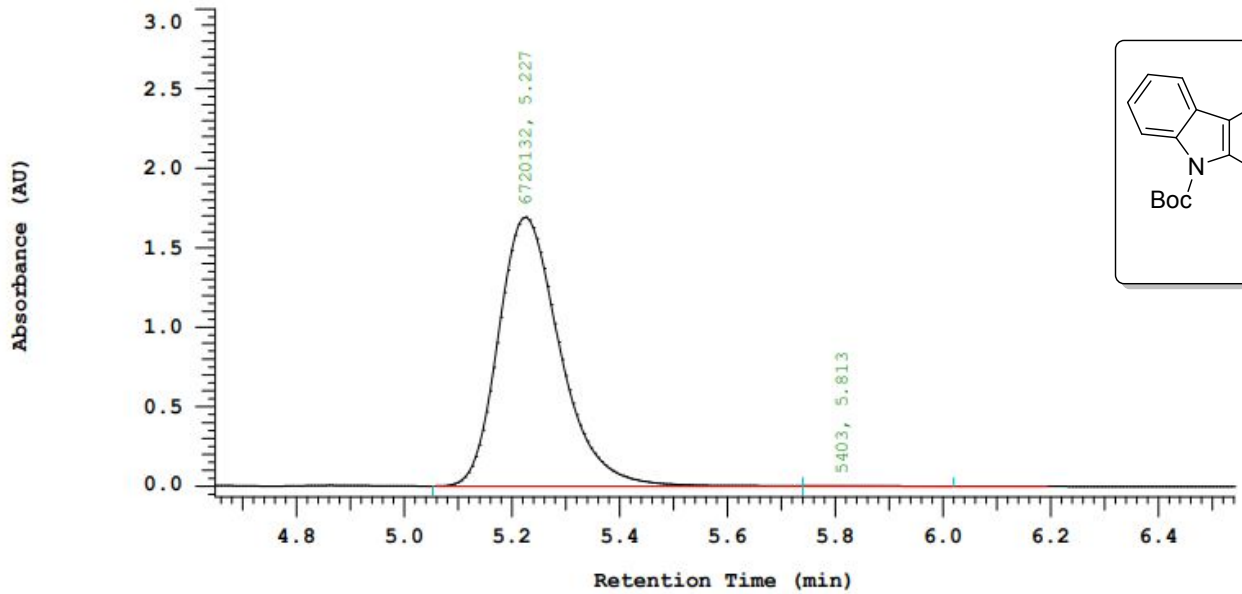

Chrom Type: Fixed WL Chromatogram, $220 \mathrm{~nm}$

Peak Quantitation: AREA

Calculation Method: AREA

\begin{tabular}{|c|c|c|c|c|}
\hline o. & $\mathrm{RT}$ & Area & Area \& & $\mathrm{BC}$ \\
\hline 1 & 5.227 & 6720132 & 99.920 & BV \\
\hline 2 & 5.813 & 5403 & 0.080 & TBB \\
\hline & & 6725535 & 100.000 & \\
\hline
\end{tabular}


The ${ }^{1} \mathrm{H}$ NMR spectrum of $6 \mathrm{n}\left(400 \mathrm{MHz}, \mathrm{CDCl}_{3}\right)$

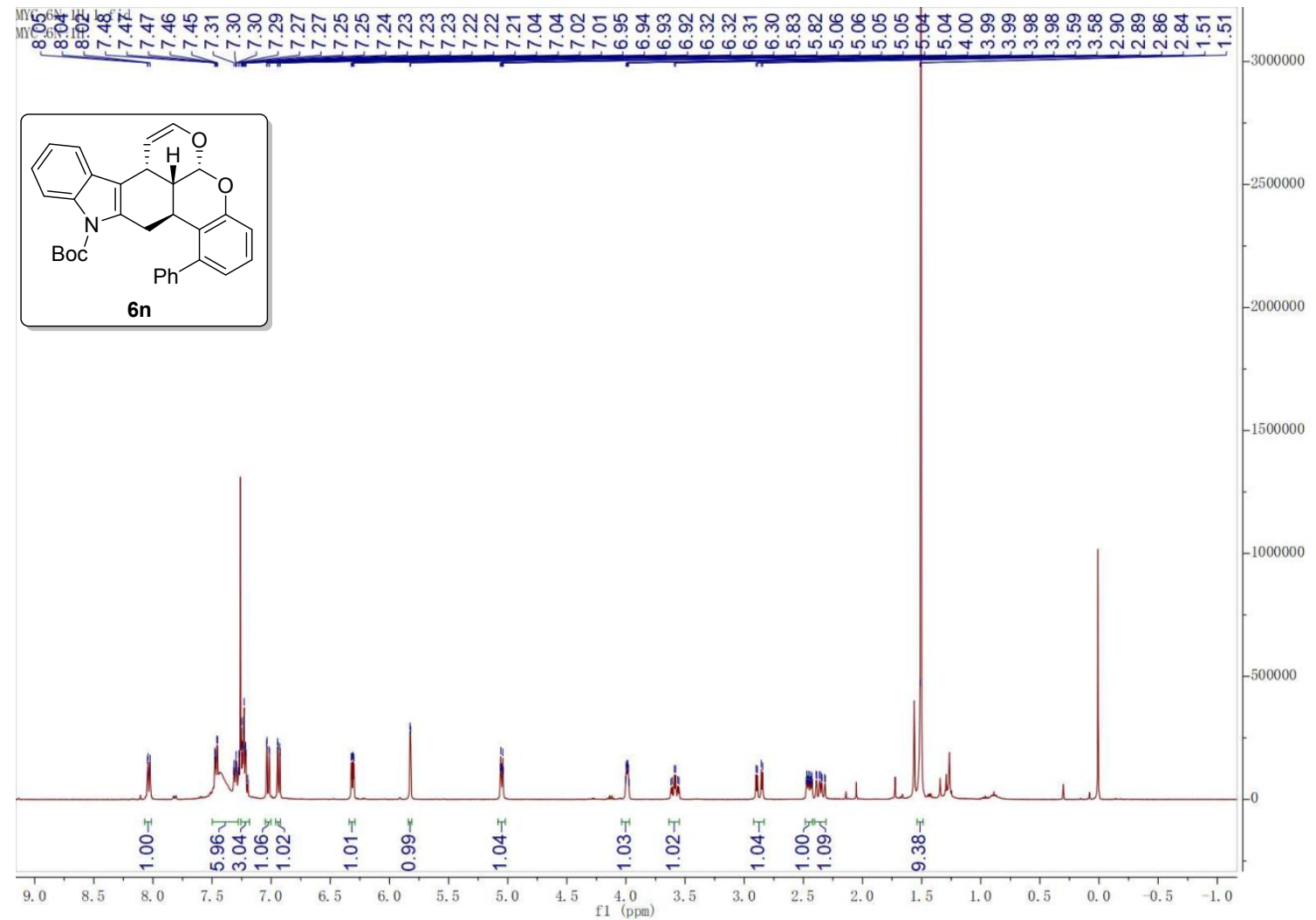

The ${ }^{13} \mathrm{C}$ NMR spectrum of $6 \mathrm{n}\left(100 \mathrm{MHz}, \mathrm{CDCl}_{3}\right)$

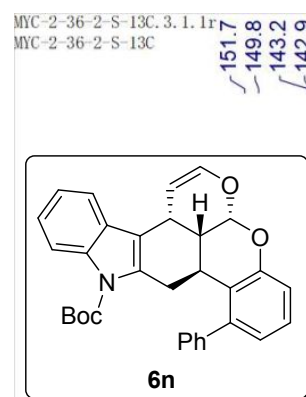

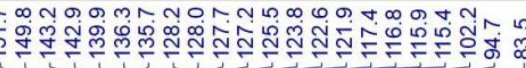

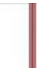




\section{The HPLC of racemic 6n}

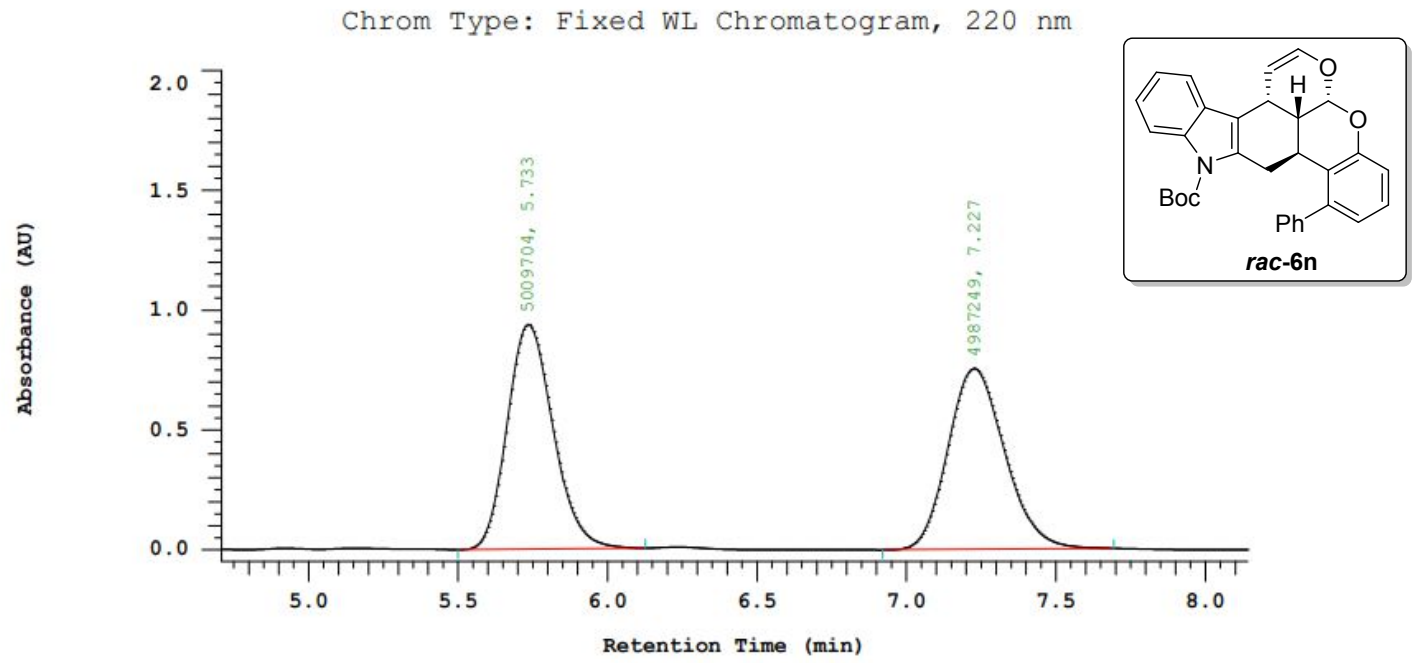

Peak Quantitation: AREA

Calculation Method: AREA음

\begin{tabular}{ccccc} 
No. & RT & Area & Area $\%$ & BC \\
\hline 1 & 5.733 & 5009704 & 50.112 & BB \\
2 & 7.227 & 4987249 & 49.888 & BB \\
\hline & & 9996953 & 100.000 & \\
\hline
\end{tabular}

\section{The HPLC of chiral 6n}

Chrom Type: Fixed WL Chromatogram, 220 nm

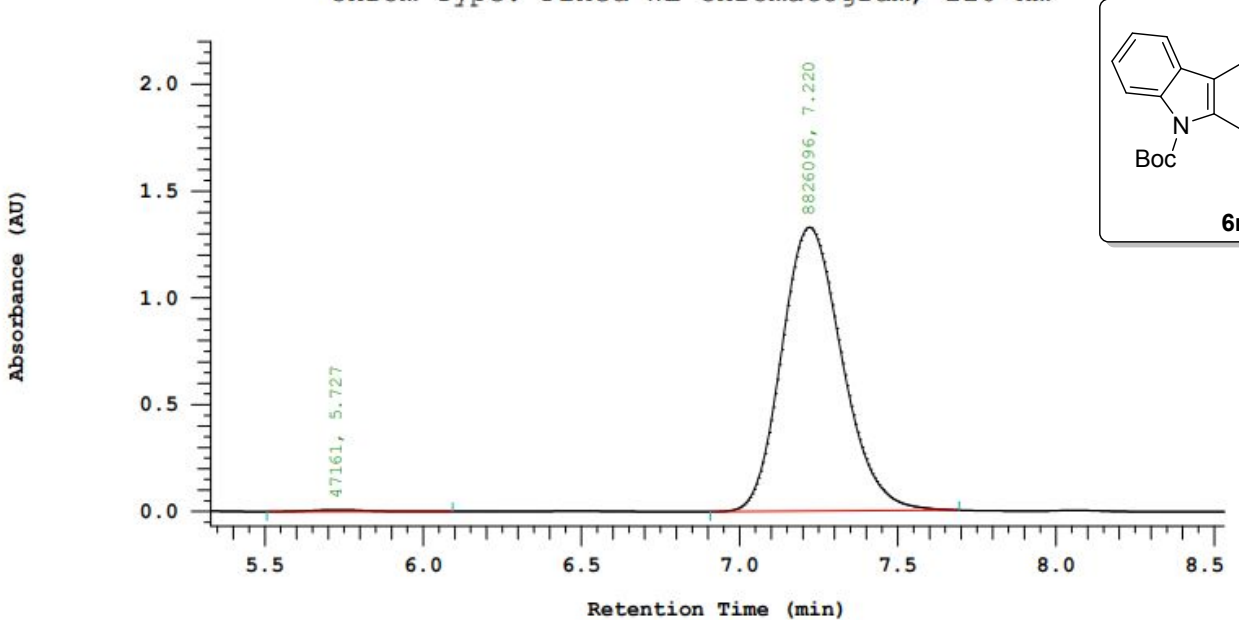

Chrom Type: Fixed WL Chromatogram, $220 \mathrm{~nm}$

Peak Quantitation: AREA

Calculation Method: AREA을

\begin{tabular}{|c|c|c|c|c|}
\hline No. & $\mathrm{RT}$ & Area & Area 웅 & $B C$ \\
\hline 1 & 5.727 & 47161 & 0.532 & BB \\
\hline \multirow[t]{2}{*}{2} & 7.220 & 8826096 & 99.468 & $\mathrm{BB}$ \\
\hline & & 8873257 & 100.000 & \\
\hline
\end{tabular}


The ${ }^{1} \mathrm{H}$ NMR spectrum of $60\left(400 \mathrm{MHz}, \mathrm{CDCl}_{3}\right)$

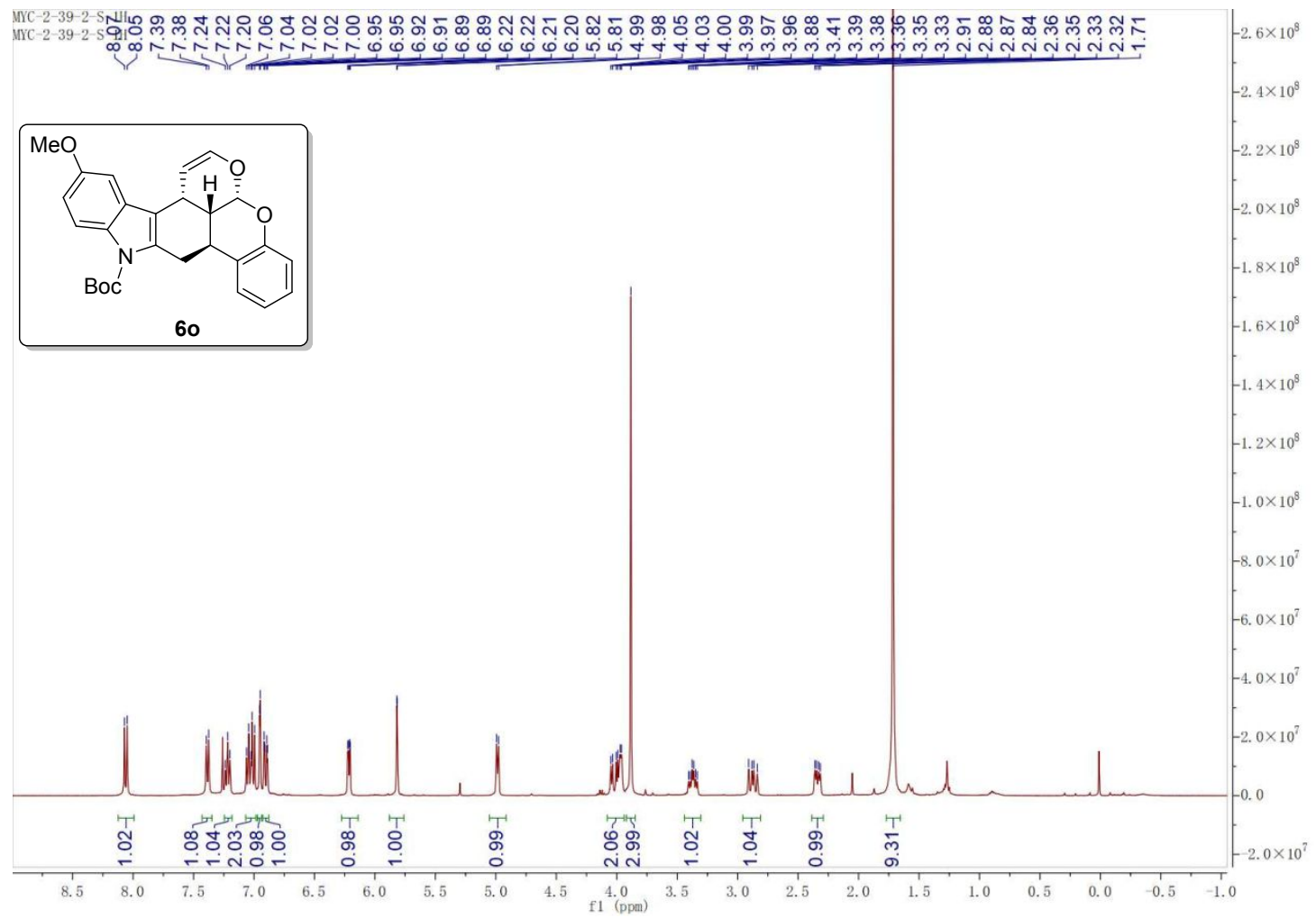

The ${ }^{13} \mathrm{C}$ NMR spectrum of $60\left(100 \mathrm{MHz}, \mathrm{CDCl}_{3}\right)$

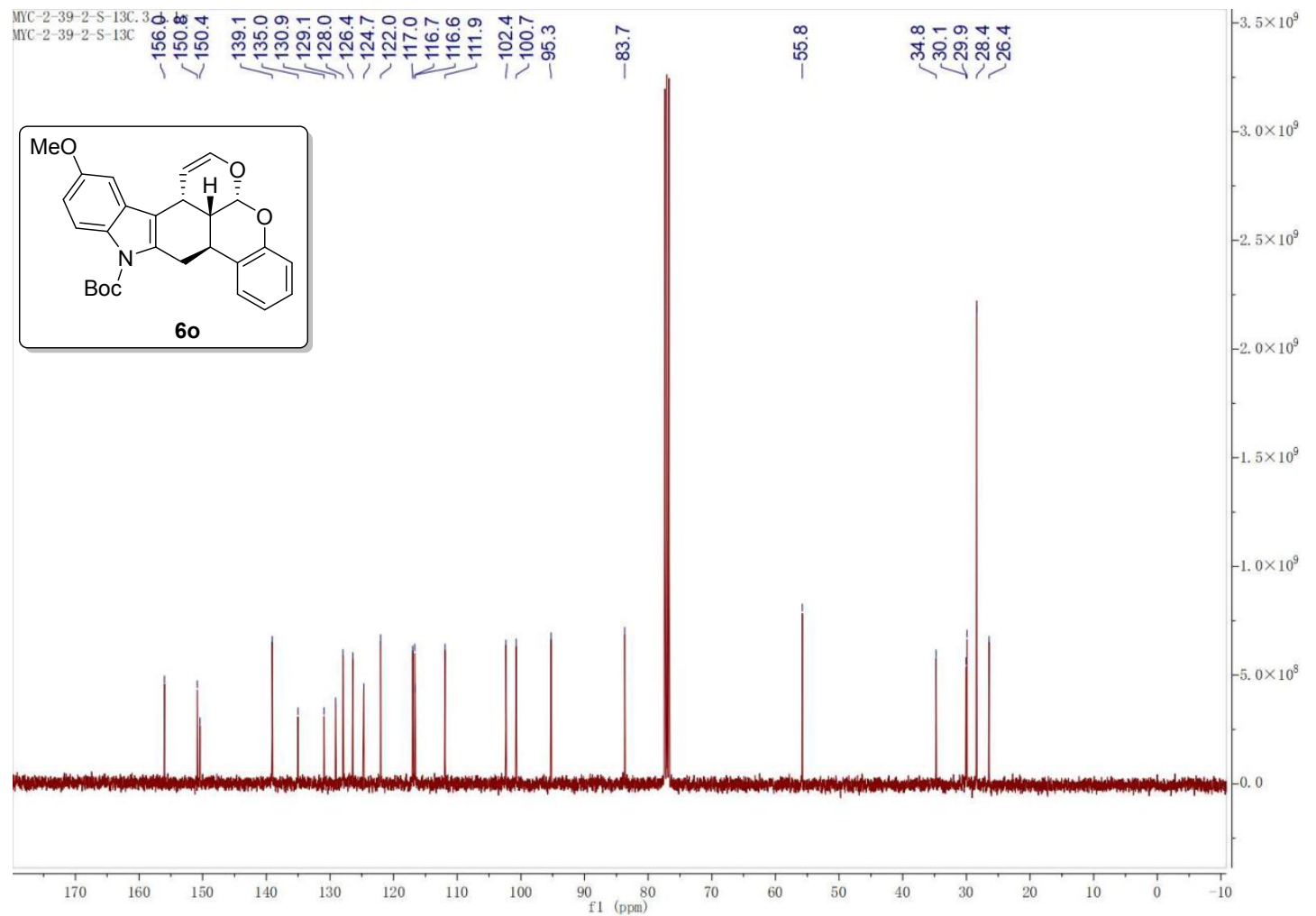




\section{The HPLC of racemic 60}

Chrom Type: Fixed WL Chromatogram, 205 nm

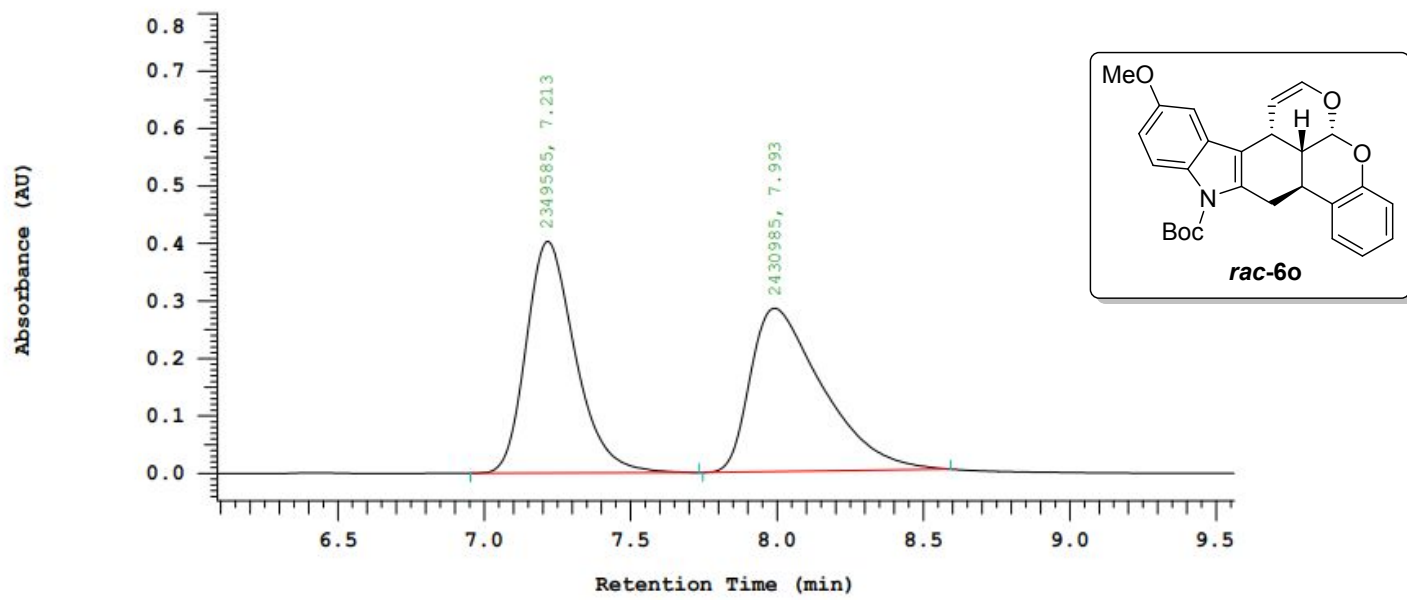

Chrom Type: Fixed WL Chromatogram, 205 nm

Peak Quantitation: AREA

Calculation Method: AREA응

\begin{tabular}{ccccc} 
No. & RT & Area & Area 8 & BC \\
\hline 1 & 7.213 & 2349585 & 49.149 & BB \\
2 & 7.993 & 2430985 & 50.851 & BB \\
\hline & & 4780570 & 100.000 & \\
\hline
\end{tabular}

The HPLC of chiral 60

Chrom Type: Fixed WL Chromatogram, 205 nm

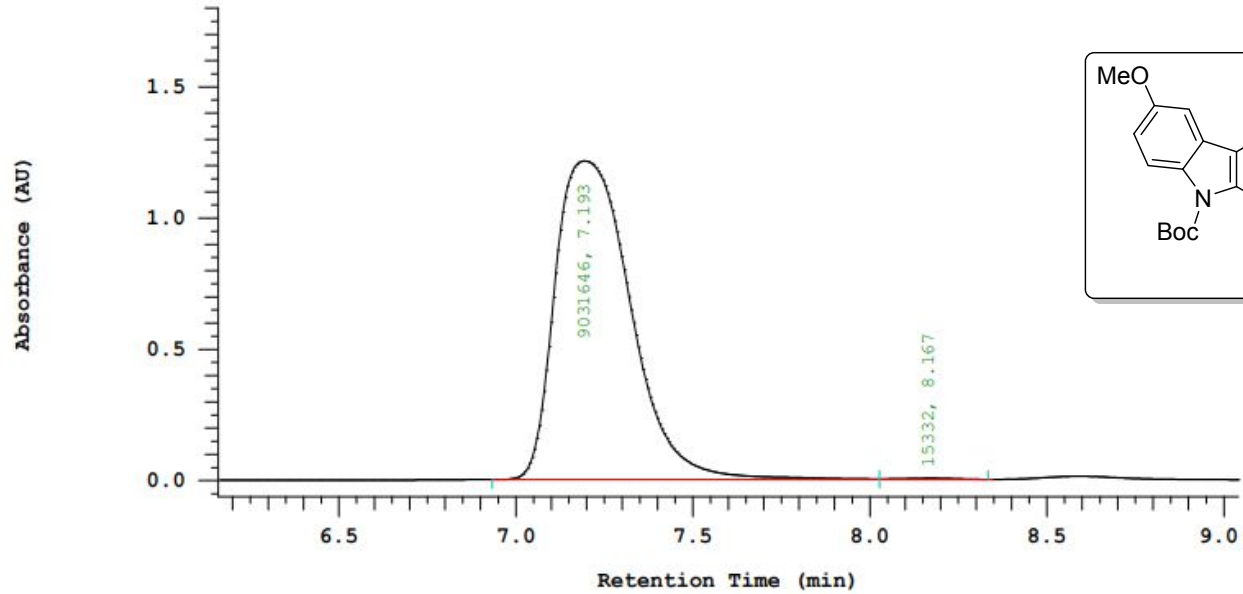

Chrom Type: Fixed WL Chromatogram, 205 nm

Peak Quantitation: AREA

Calculation Method: AREA용

\begin{tabular}{rrrrr} 
No. & RT & Area & Area & BC \\
\hline 1 & 7.193 & 9031646 & 99.831 & BV \\
2 & 8.167 & 15332 & 0.169 & TBB \\
\hline & & 9046978 & 100.000 & \\
\hline
\end{tabular}


The ${ }^{1} \mathrm{H}$ NMR spectrum of $6 \mathrm{p}\left(400 \mathrm{MHz}, \mathrm{CDCl}_{3}\right)$

Mต్ల

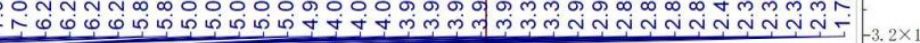

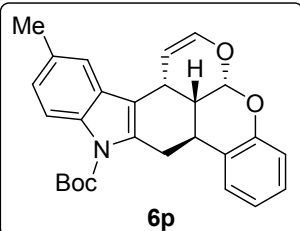

$6 p$

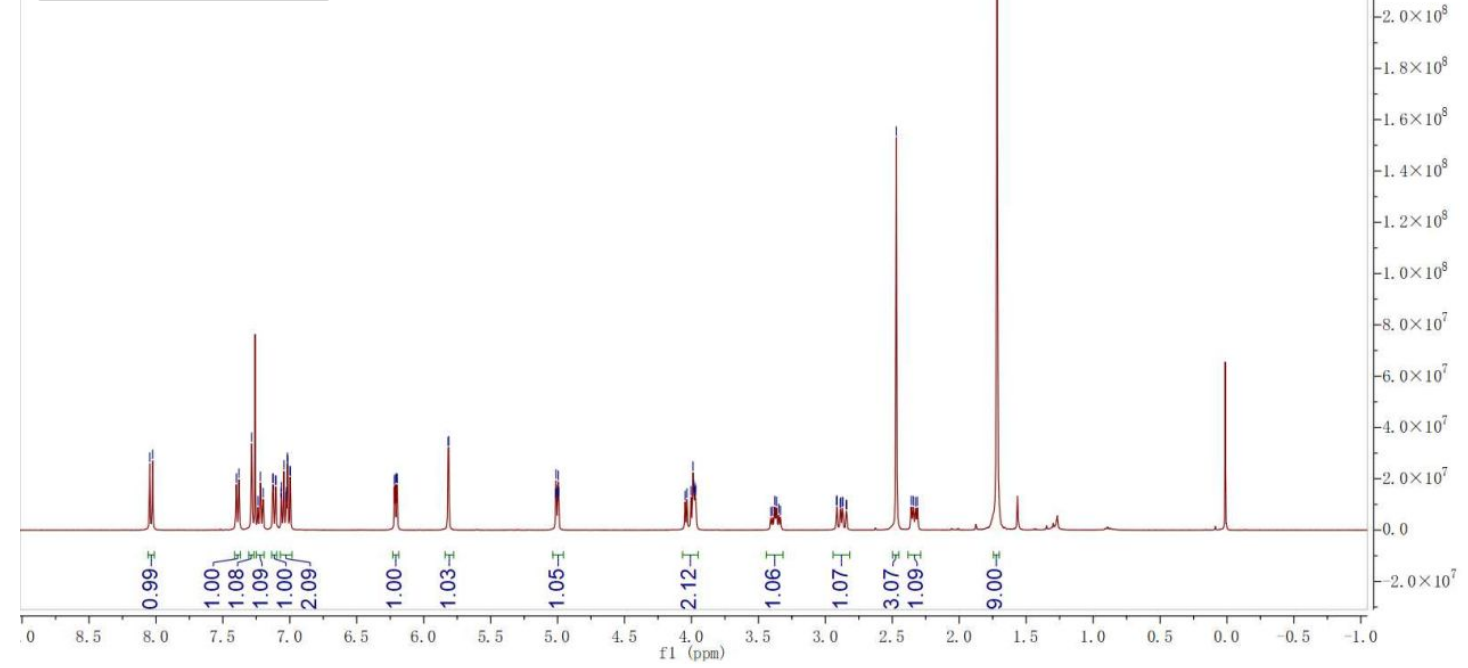

The ${ }^{13} \mathrm{C}$ NMR spectrum of $6 \mathrm{p}\left(100 \mathrm{MHz}, \mathrm{CDCl}_{3}\right)$

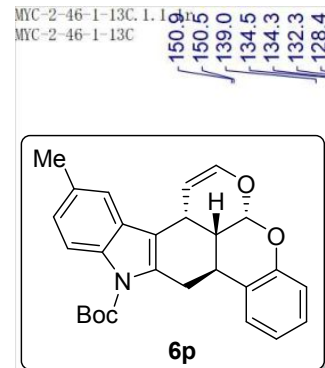

$6 p$

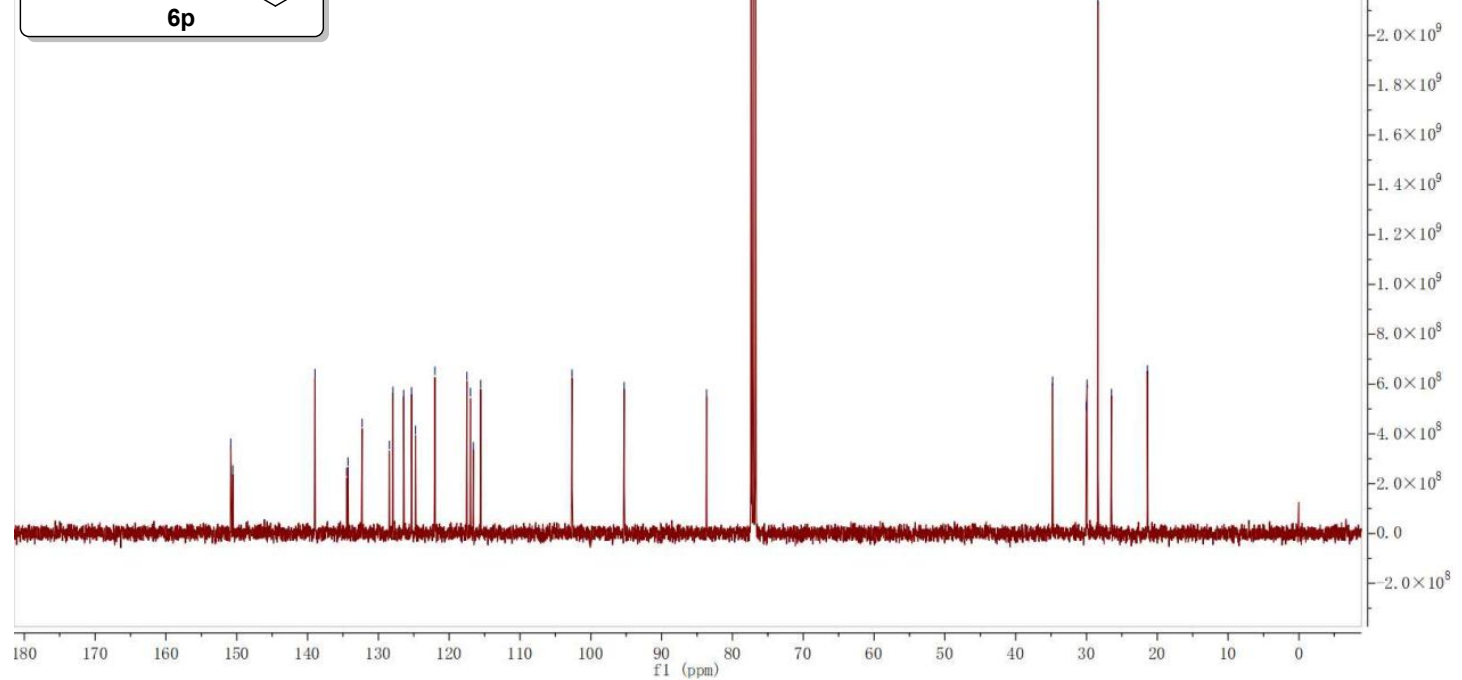




\section{The HPLC of racemic $6 p$}

Chrom Type: Fixed WL Chromatogram, $205 \mathrm{~nm}$

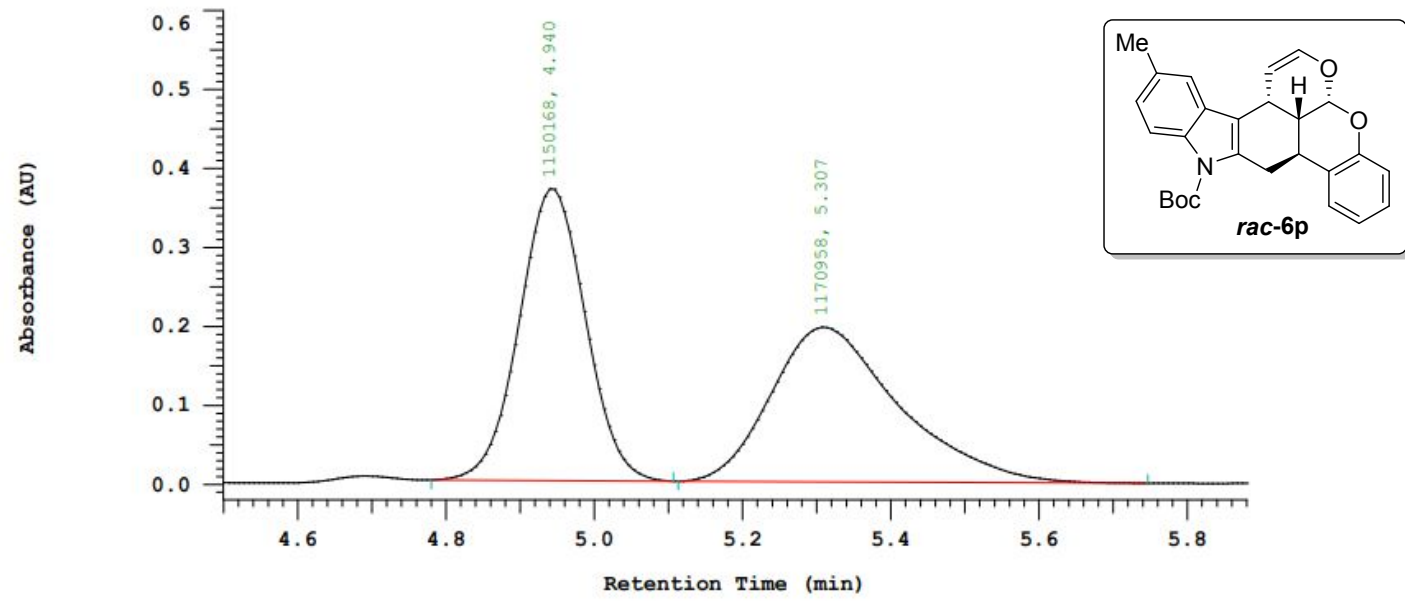

Chrom Type: Fixed WL Chromatogram, $205 \mathrm{~nm}$ Peak Quantitation: AREA

Calculation Method: AREA음

\begin{tabular}{ccccc} 
No. & RT & Area & Area & BC \\
\hline 1 & 4.940 & 1150168 & 49.552 & BB \\
2 & 5.307 & 1170958 & 50.448 & BB \\
\hline & 2321126 & 100.000 & \\
\hline
\end{tabular}

\section{The HPLC of chiral 6p}

Chrom Type: Fixed WL Chromatogram, 205 nm

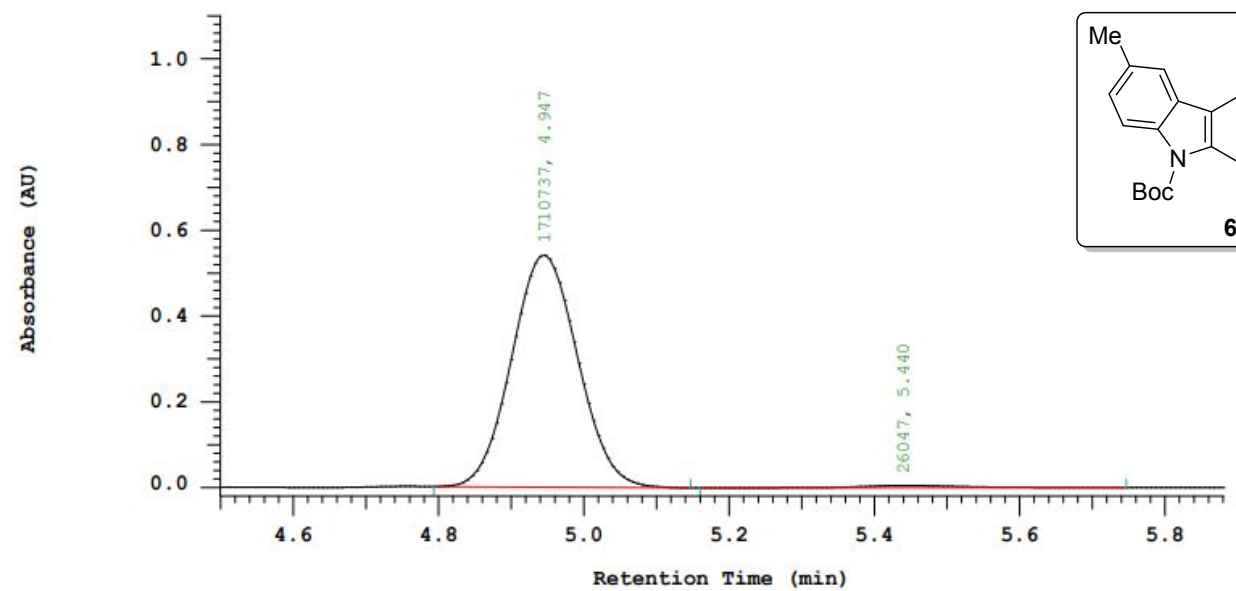

Chrom Type: Fixed WL Chromatogram, $205 \mathrm{~nm}$

Peak Quantitation: AREA

Calculation Method: AREA용

\begin{tabular}{rrrrr} 
No. & RT & Area & Area & BC \\
\hline 1 & 4.947 & 1710737 & 98.500 & BB \\
2 & 5.440 & 26047 & 1.500 & BB \\
\hline & & 1736784 & 100.000 &
\end{tabular}


The ${ }^{1} \mathrm{H}$ NMR spectrum of $6 \mathrm{q}\left(400 \mathrm{MHz}, \mathrm{CDCl}_{3}\right)$

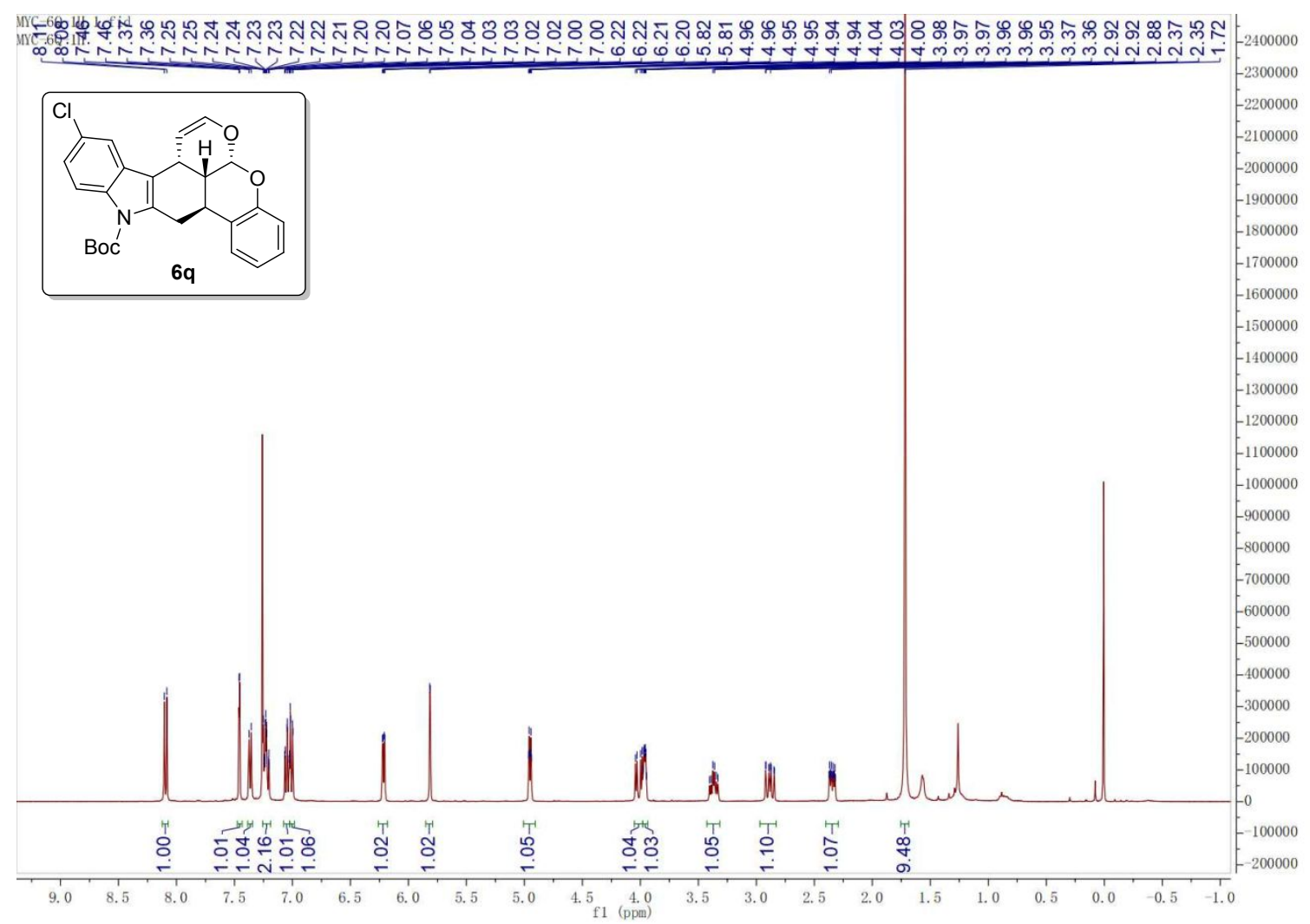

The ${ }^{13} \mathrm{C}$ NMR spectrum of $6 \mathrm{q}\left(100 \mathrm{MHz}, \mathrm{CDCl}_{3}\right)$

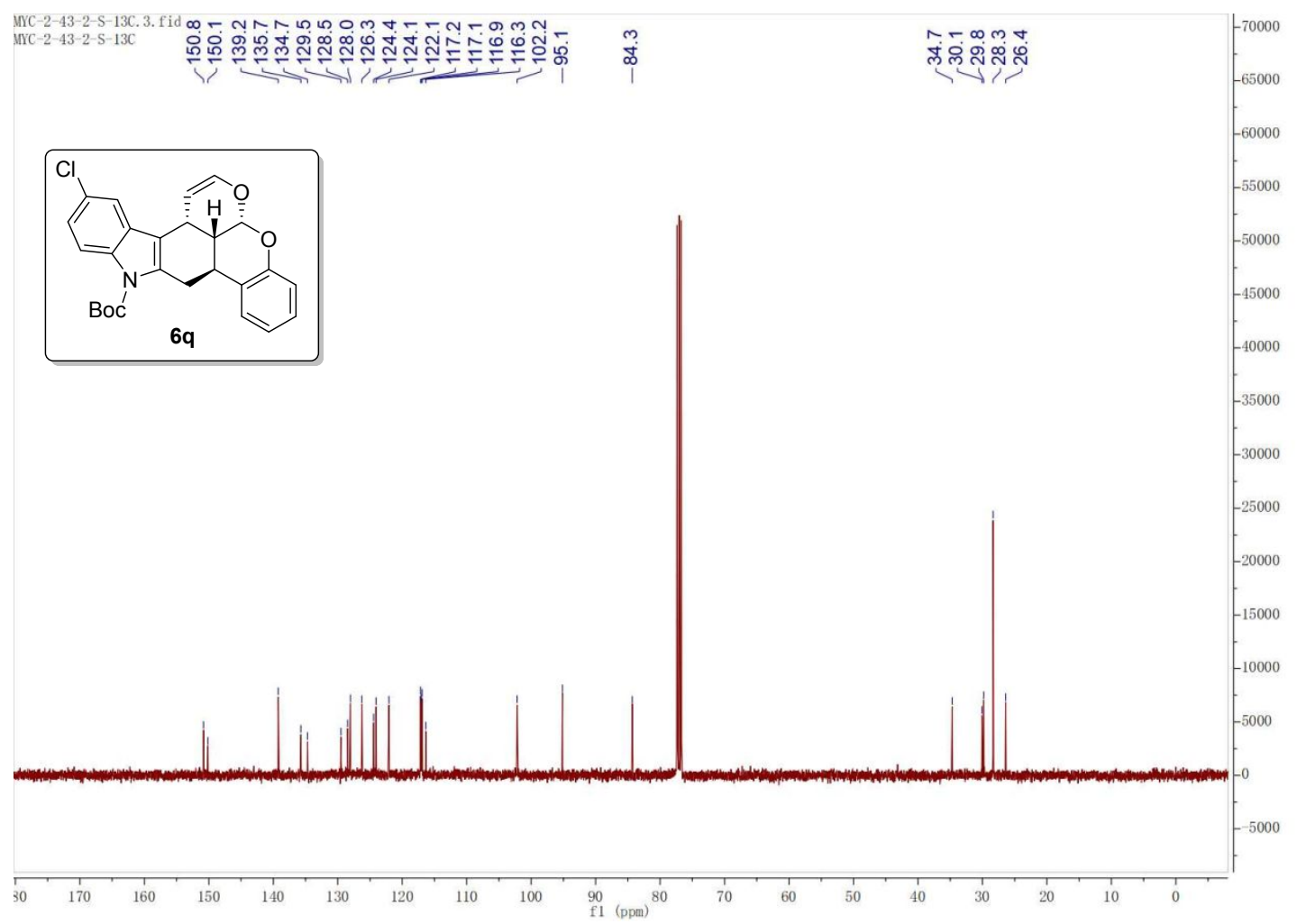




\section{The HPLC of racemic $6 q$}

Chrom Type: Fixed WL Chromatogram, 205 nm

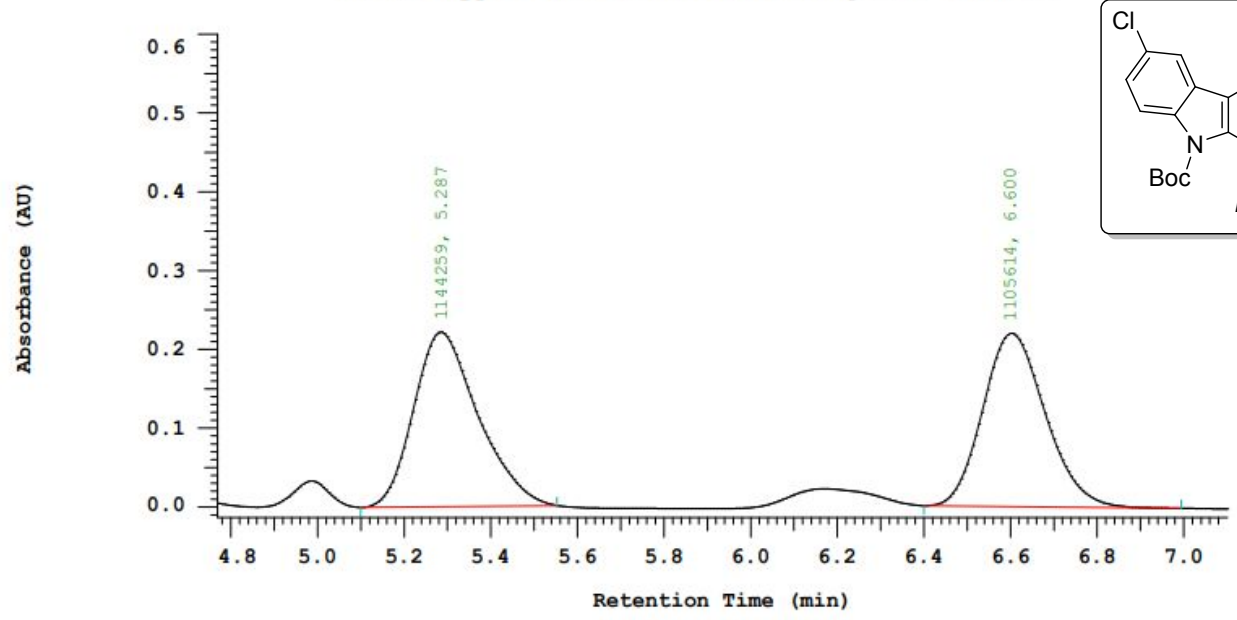

Chrom Type: Fixed WL Chromatogram, 205 nm

Peak Quantitation: AREA

Calculation Method: AREA응

\begin{tabular}{ccccc} 
No. & RT & Area & Area & BC \\
\hline 1 & 5.287 & 1144259 & 50.859 & BB \\
2 & 6.600 & 1105614 & 49.141 & BB \\
\hline & & 2249873 & 100.000 & \\
\hline
\end{tabular}

\section{The HPLC of chiral 6q}

Chrom Type: Fixed WL Chromatogram, 205 nm

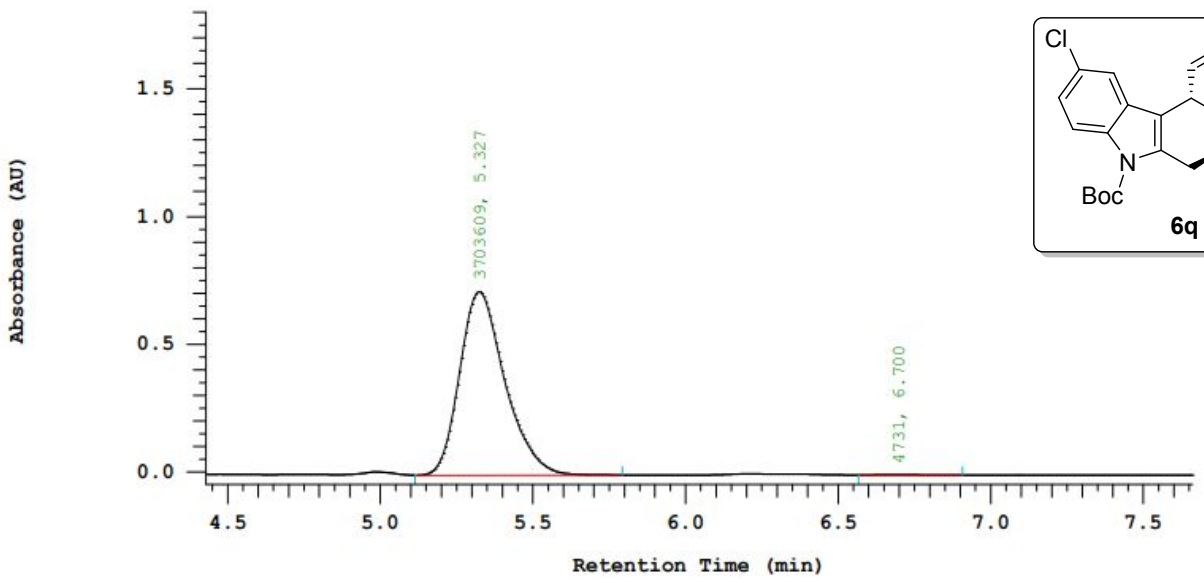

Chrom Type: Fixed WL Chromatogram, $205 \mathrm{~nm}$

Peak Quantitation: AREA

Calculation Method: AREA응

\begin{tabular}{rrrrr} 
No. & RT & Area & Area & BC \\
\hline 1 & 5.327 & 3703609 & 99.872 & BB \\
2 & 6.700 & 4731 & 0.128 & BB \\
\hline & & 3708340 & 100.000 & \\
\hline
\end{tabular}


The ${ }^{1} \mathrm{H}$ NMR spectrum of $6 \mathrm{r}\left(400 \mathrm{MHz}, \mathrm{CDCl}_{3}\right)$

MG2N6\%

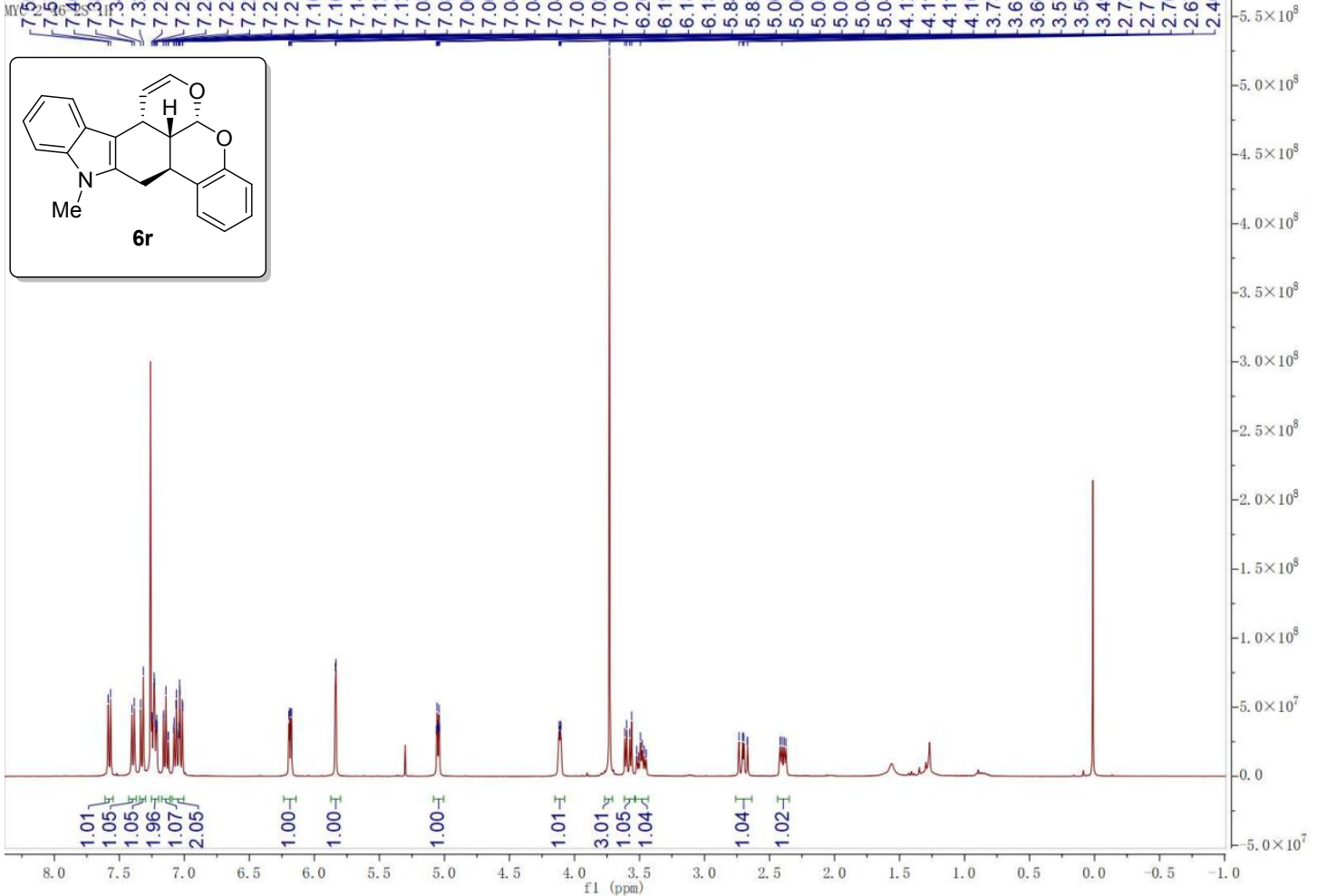

The ${ }^{13} \mathrm{C}$ NMR spectrum of $6 \mathrm{r}\left(100 \mathrm{MHz}, \mathrm{CDCl}_{3}\right)$

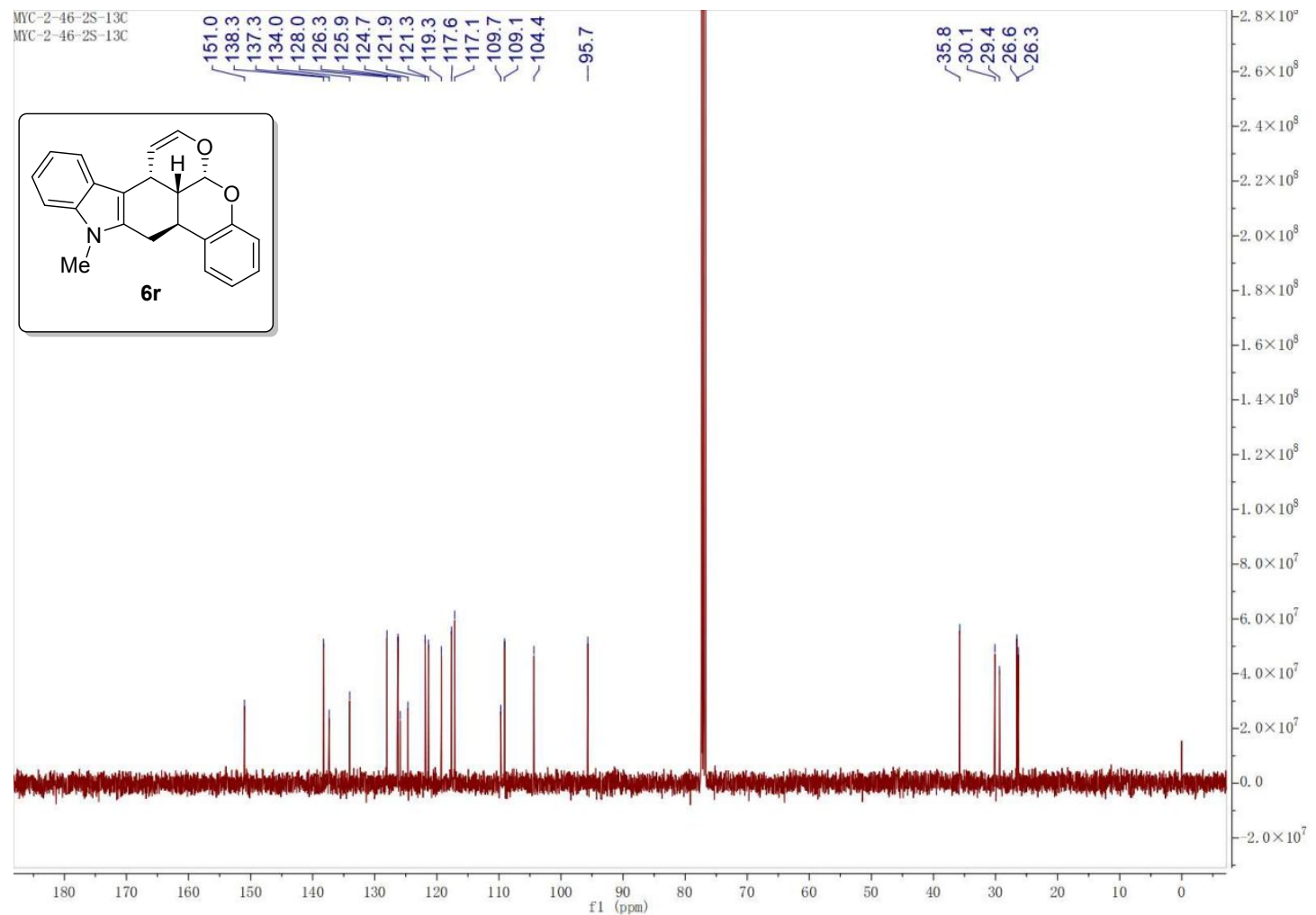




\section{The HPLC of racemic $6 r$}

Chrom Type: Fixed WL Chromatogram, 205 nm

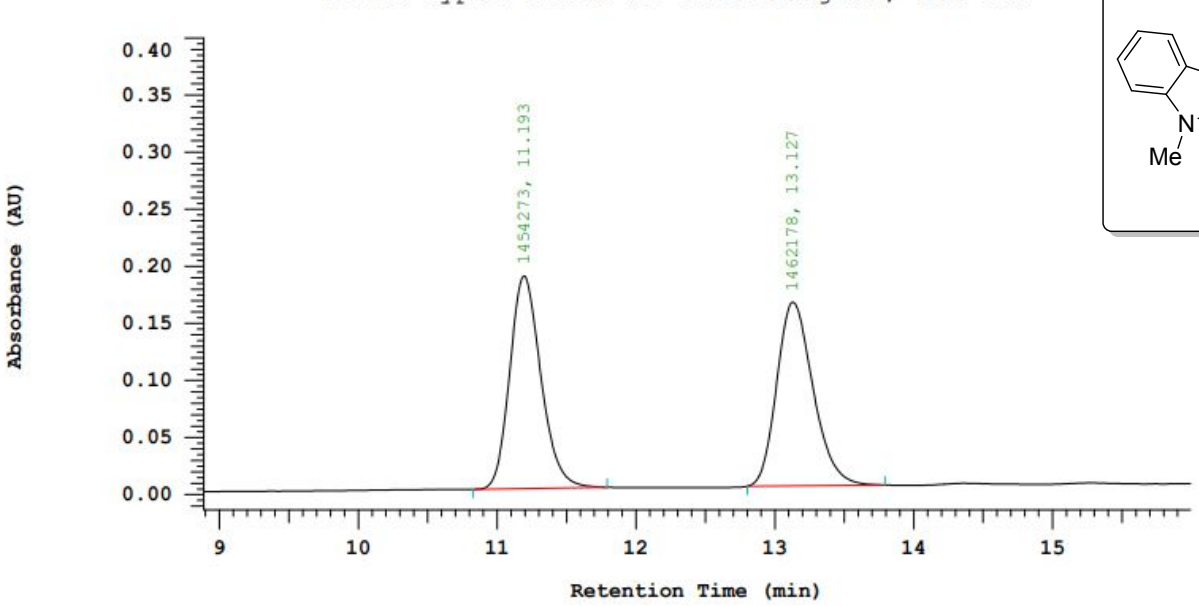

Chrom Type: Fixed WL Chromatogram, 205 nm

Peak Quantitation: AREA

Calculation Method: AREA

\begin{tabular}{ccccc} 
No. & RT & Area & Area $\%$ & BC \\
\hline 1 & 11.193 & 1454273 & 49.864 & BB \\
2 & 13.127 & 1462178 & 50.136 & BB \\
\hline & & 2916451 & 100.000 &
\end{tabular}

\section{The HPLC of chiral $6 r$}

Chrom Type: Fixed WL Chromatogram, 205 nm

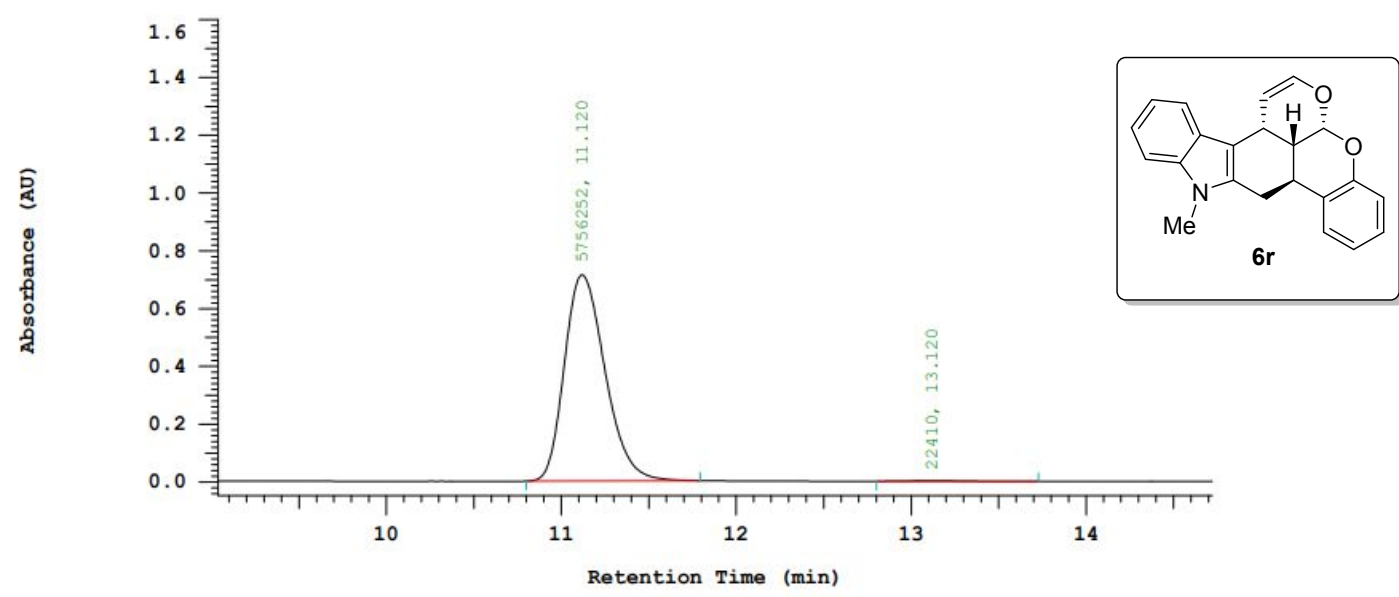

Chrom Type: Fixed WL Chromatogram, $205 \mathrm{~nm}$

Peak Quantitation: AREA

Calculation Method: AREA용

\begin{tabular}{|c|c|c|c|c|}
\hline No. & RT & Area & Area 웅 & $\mathrm{BC}$ \\
\hline 1 & 11.120 & 5756252 & 99.612 & $\mathrm{BB}$ \\
\hline 2 & 13.120 & 22410 & 0.388 & $\mathrm{BB}$ \\
\hline & & 5778662 & 100.000 & \\
\hline
\end{tabular}


The ${ }^{1} \mathrm{H}$ NMR spectrum of $6 \mathrm{~s}\left(400 \mathrm{MHz}, \mathrm{CDCl}_{3}\right)$

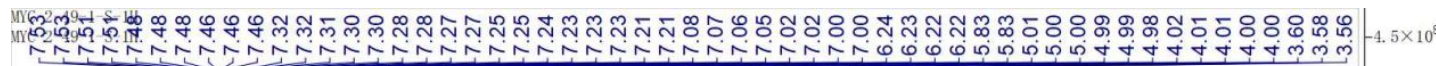

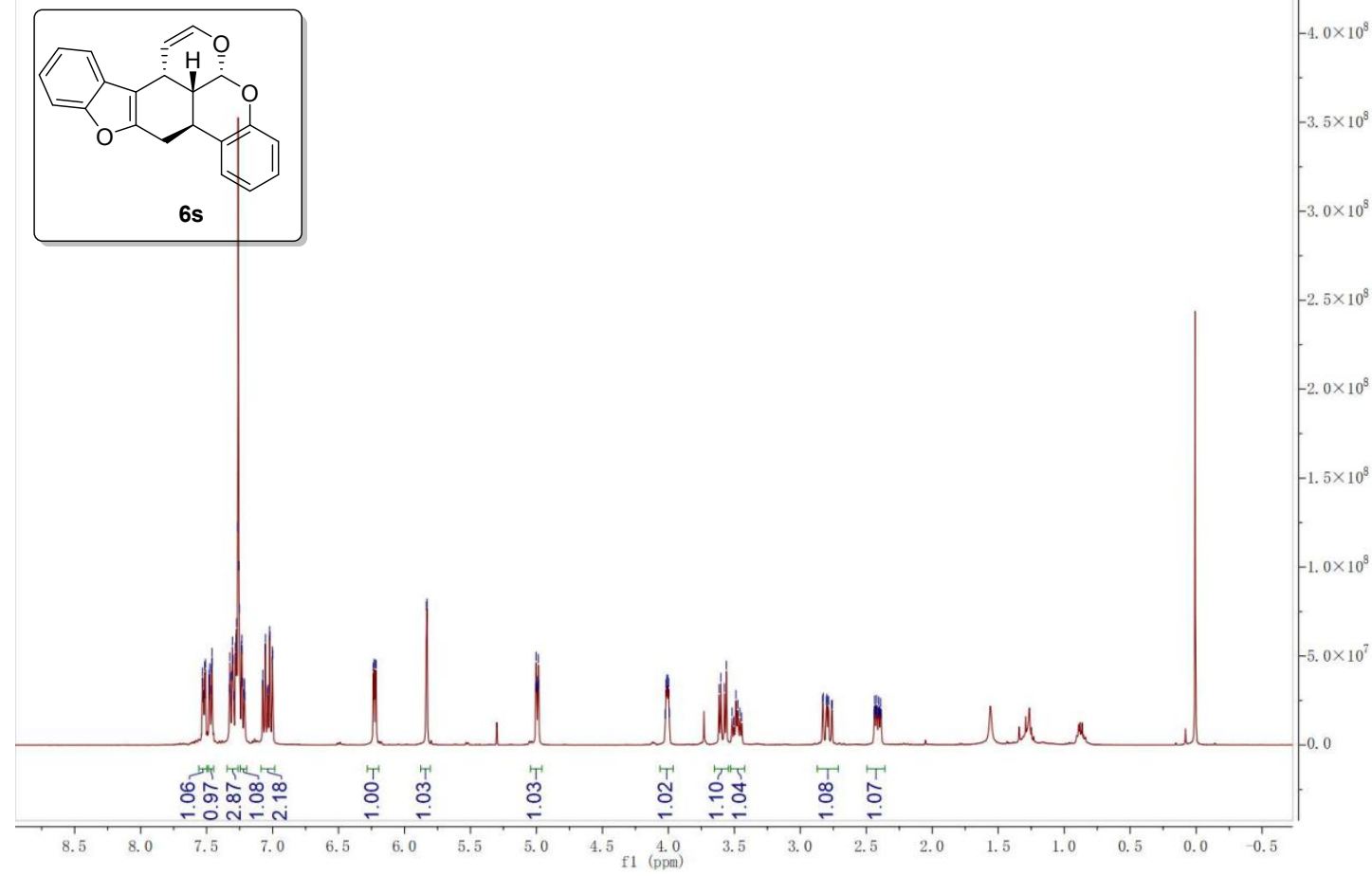

The ${ }^{13} \mathrm{C}$ NMR spectrum of $6 \mathrm{~s}\left(100 \mathrm{MHz}, \mathrm{CDCl}_{3}\right)$
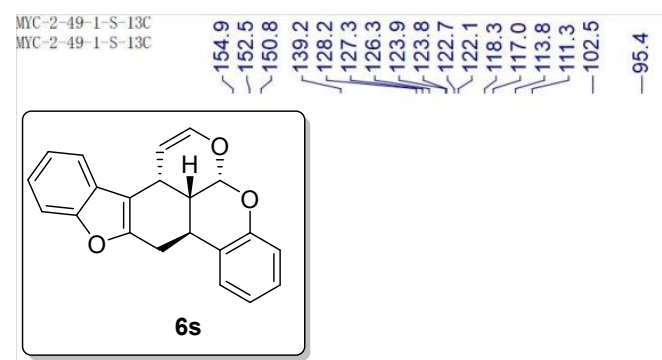

\section{:}
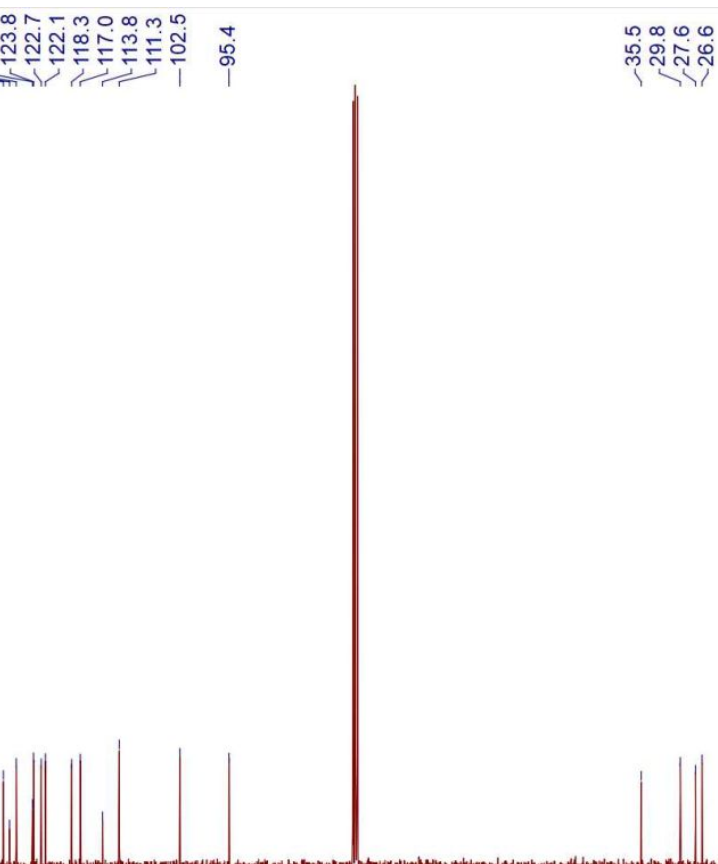

$.0 \times 10^{8}$
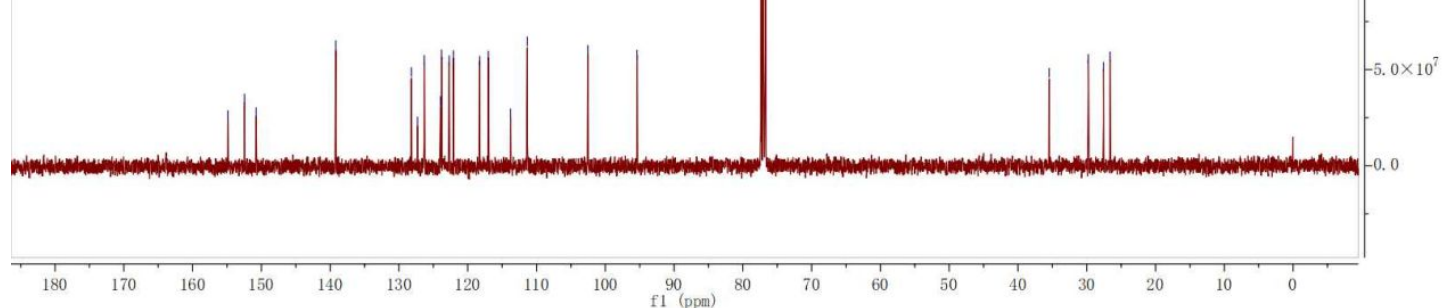


\section{The HPLC of racemic $6 \mathrm{~s}$}

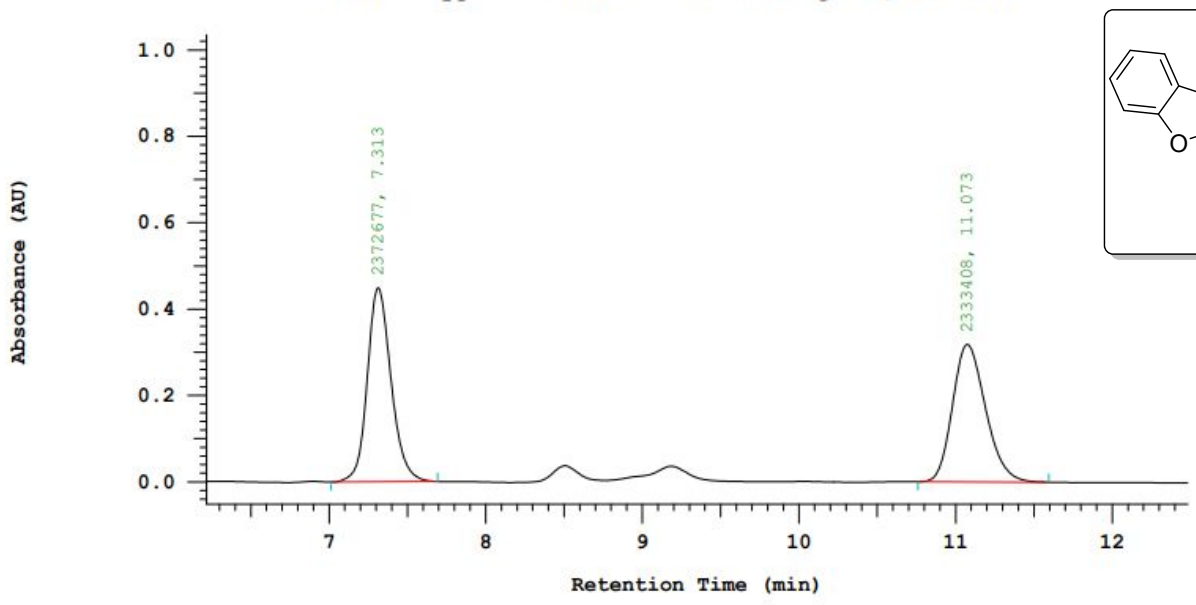

Chrom Type: Fixed WL Chromatogram, 205 nm

Peak Quantitation: AREA

Calculation Method: AREA용

\begin{tabular}{rrrrr} 
No. & \multicolumn{1}{c}{ RT } & Area & Area \% & BC \\
\hline 1 & 7.313 & 2372677 & 50.417 & BB \\
2 & 11.073 & 2333408 & 49.583 & BB \\
\hline & & 4706085 & 100.000 &
\end{tabular}

\section{The HPLC of chiral 6s}

Chrom Type: Fixed WL Chromatogram, 205 nm

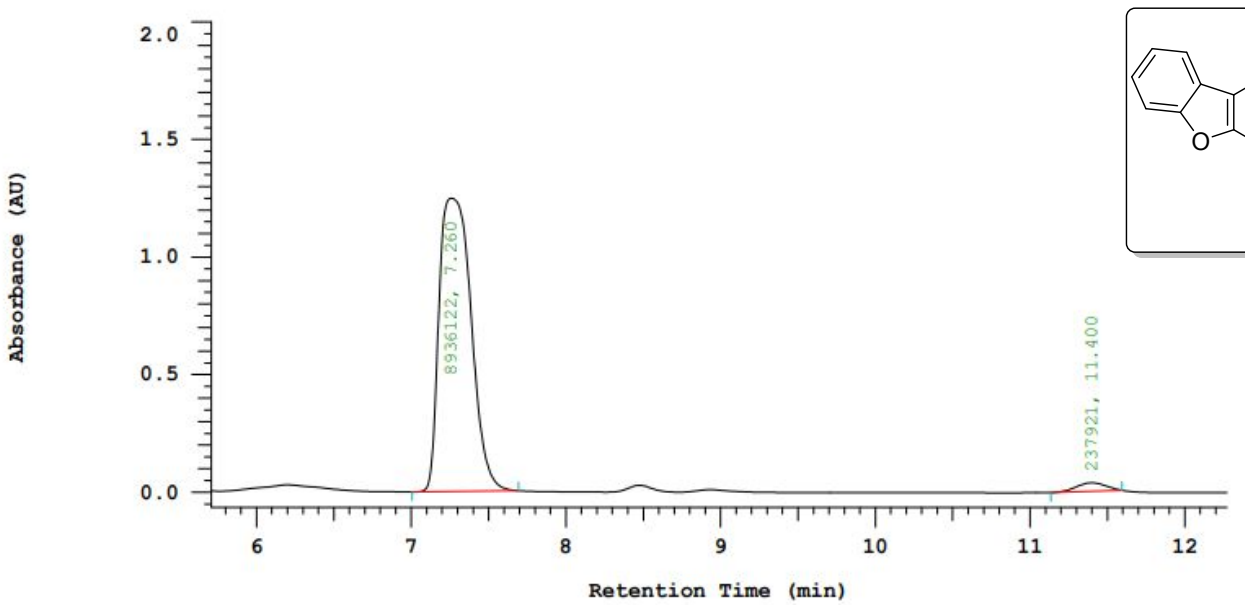

Chrom Type: Fixed WL Chromatogram, 205 nm Peak Quantitation: AREA

Calculation Method: AREA응

\begin{tabular}{|c|c|c|c|c|}
\hline No. & RT & Area & Area \& & $\mathrm{BC}$ \\
\hline 1 & 7.260 & 8936122 & 97.407 & $\mathrm{BB}$ \\
\hline \multirow[t]{2}{*}{2} & 11.400 & 237921 & 2.593 & $\mathrm{BB}$ \\
\hline & & 9174043 & 100.000 & \\
\hline
\end{tabular}


The ${ }^{1} \mathrm{H}$ NMR spectrum of $6 \mathrm{t}\left(400 \mathrm{MHz}, \mathrm{CDCl}_{3}\right)$

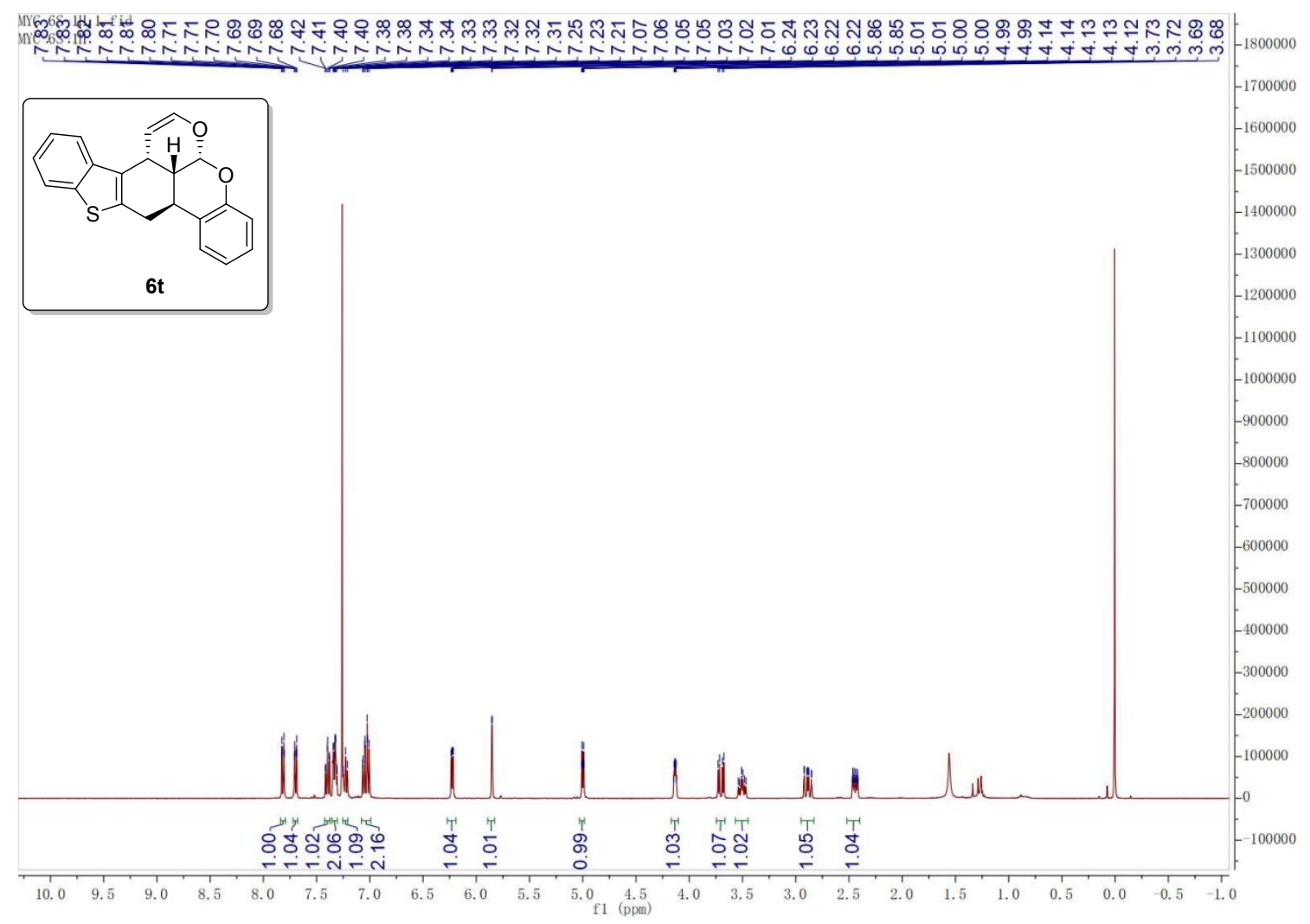

The ${ }^{13} \mathrm{C}$ NMR spectrum of $6 \mathrm{t}\left(100 \mathrm{MHz}, \mathrm{CDCl}_{3}\right)$

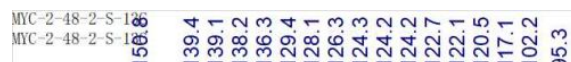

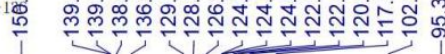

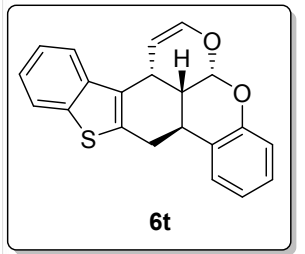


The HPLC of racemic $6 t$

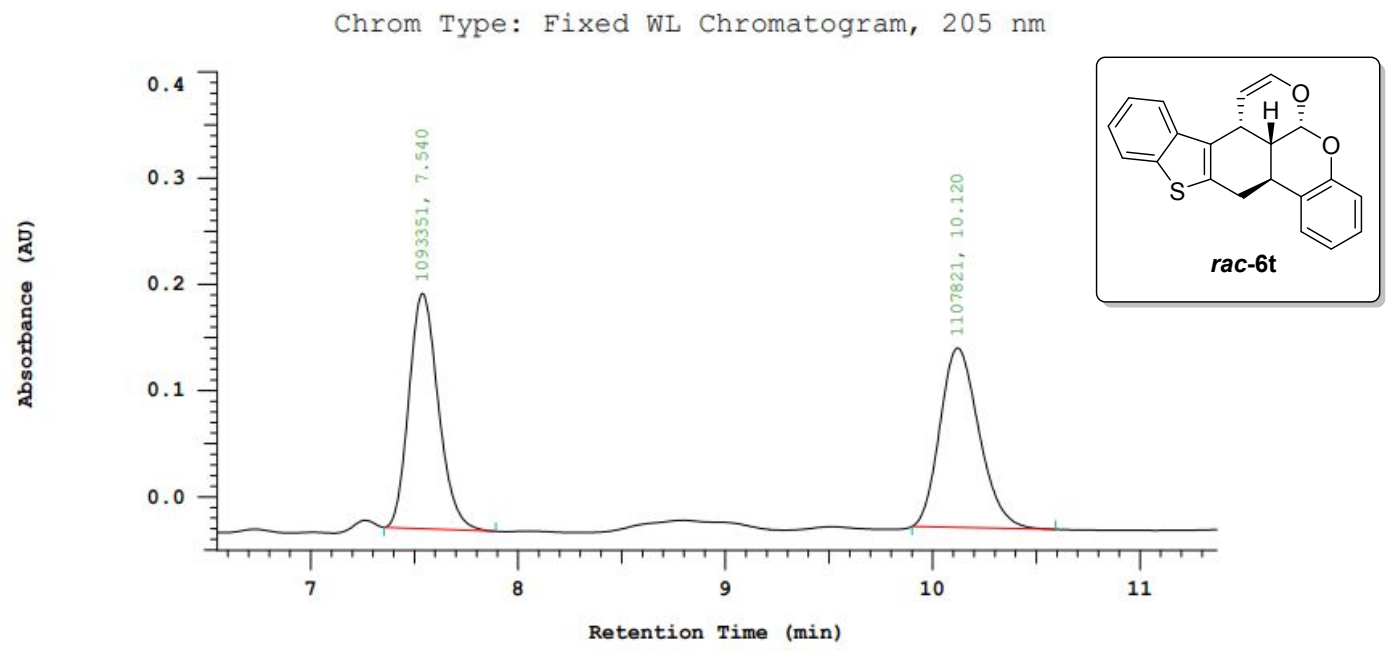

Peak Quantitation: AREA

Chrom Type: Fixed WL Chromatogram, $205 \mathrm{~nm}$ Calculation Method: AREA

\begin{tabular}{rrrrr} 
No. & RT & Area & Area & BC \\
\hline 1 & 7.540 & 1093351 & 49.671 & BB \\
2 & 10.120 & 1107821 & 50.329 & BB \\
\hline & & 2201172 & 100.000 & \\
\hline
\end{tabular}

\section{The HPLC of chiral 6t}

Chrom Type: Fixed WL Chromatogram, 205 nm

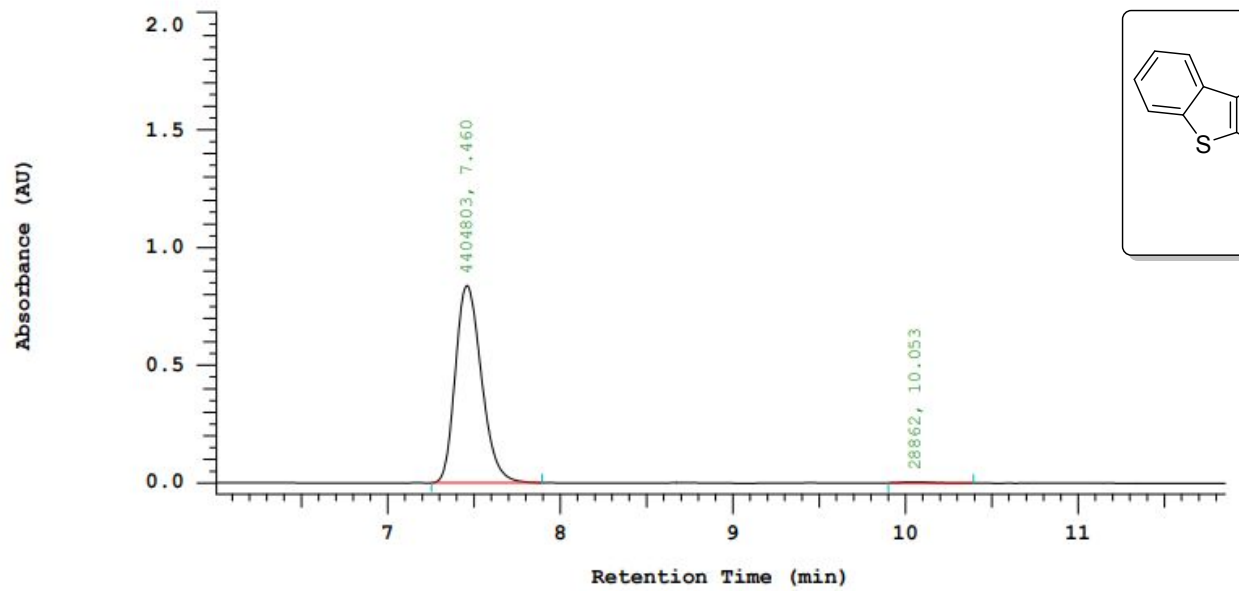

Chrom Type: Fixed WL Chromatogram, $205 \mathrm{~nm}$ Peak Quantitation: AREA Calculation Method: AREA용

\begin{tabular}{rrrrr} 
No. & \multicolumn{1}{c}{ RT } & Area & Area & BC \\
\hline 1 & 7.460 & 4404803 & 99.349 & BB \\
2 & 10.053 & 28862 & 0.651 & BB \\
\hline & & 4433665 & 100.000 & \\
\hline
\end{tabular}


The ${ }^{1} \mathrm{H}$ NMR spectrum of $8\left(400 \mathrm{MHz}, \mathrm{CDCl}_{3}\right)$

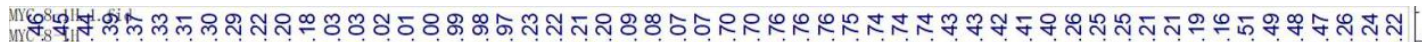

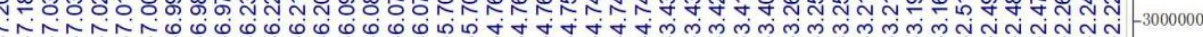
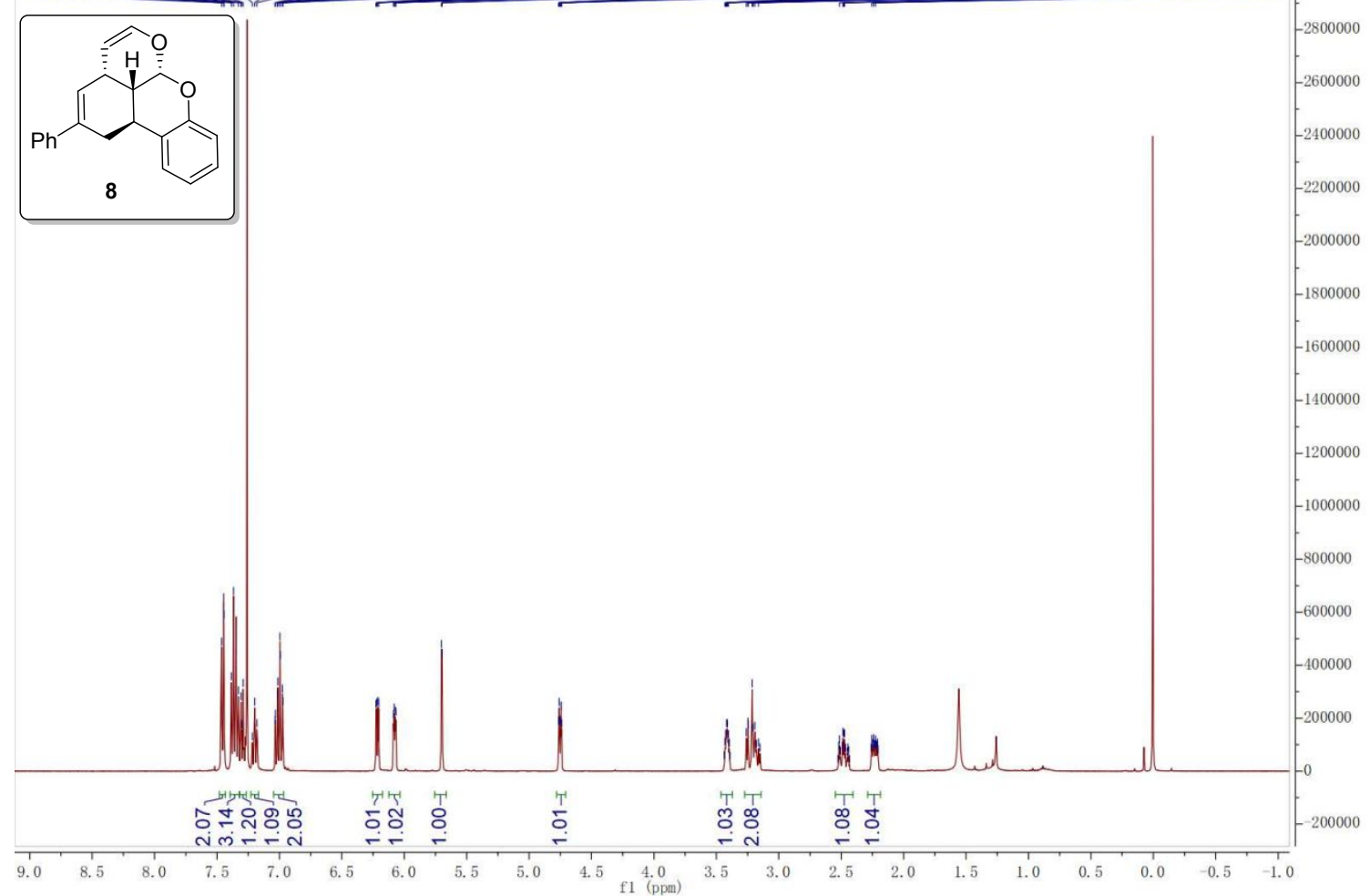

The ${ }^{13} \mathrm{C}$ NMR spectrum of $8\left(100 \mathrm{MHz}, \mathrm{CDCl}_{3}\right)$

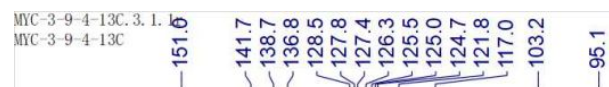

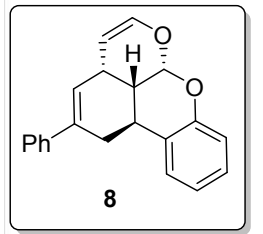

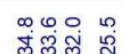
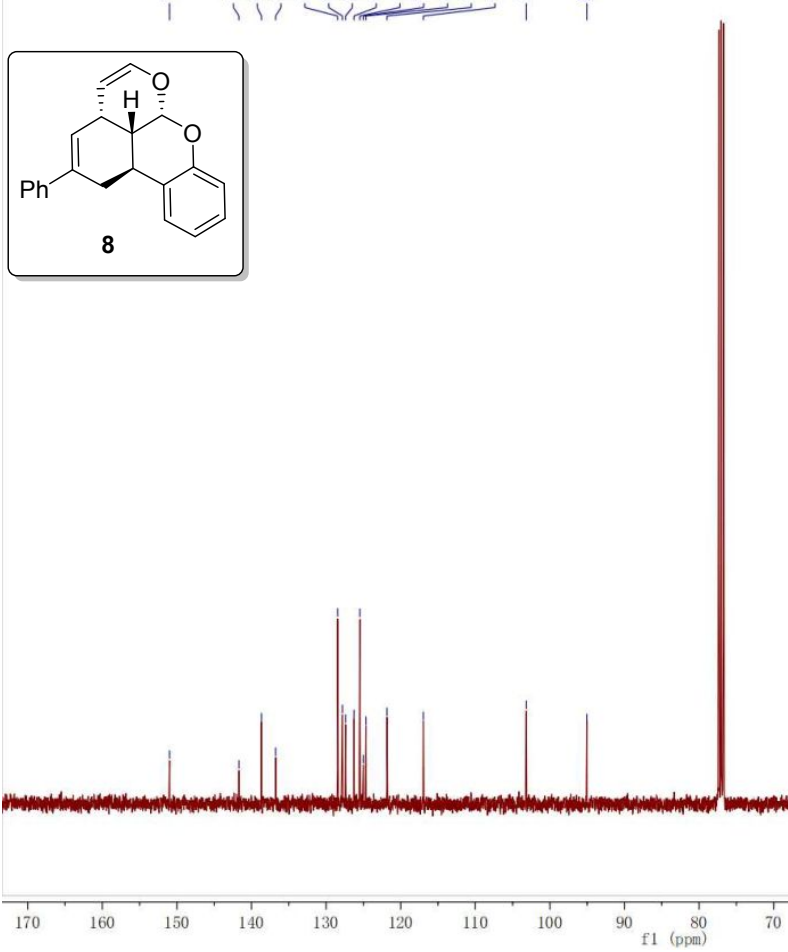


\section{The HPLC of racemic 8}

Chrom Type: Fixed WL Chromatogram, 205 nm

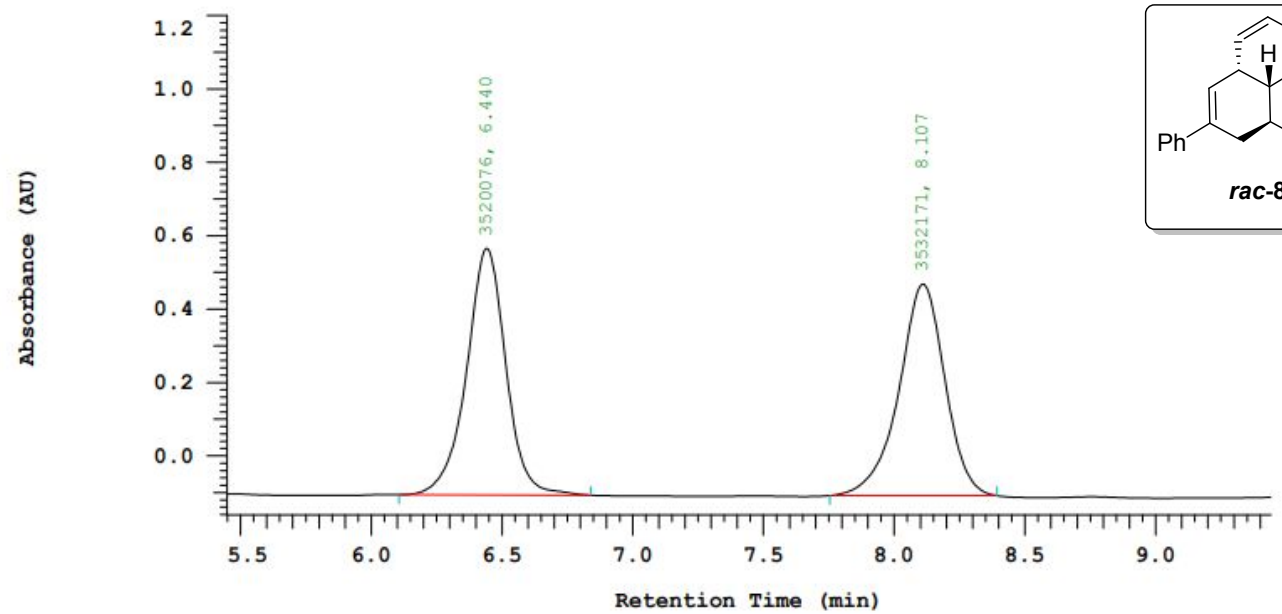

Chrom Type: Fixed WL Chromatogram, $205 \mathrm{~nm}$

Peak Quantitation: AREA

Calculation Method: AREA응

\begin{tabular}{ccccc} 
No. & RT & Area & Area s & BC \\
\hline 1 & 6.440 & 3520076 & 49.914 & BB \\
2 & 8.107 & 3532171 & 50.086 & BB \\
\hline & & 7052247 & 100.000 & \\
\hline
\end{tabular}

\section{The HPLC of chiral 8}

Chrom Type: Fixed WL Chromatogram, 205 nm

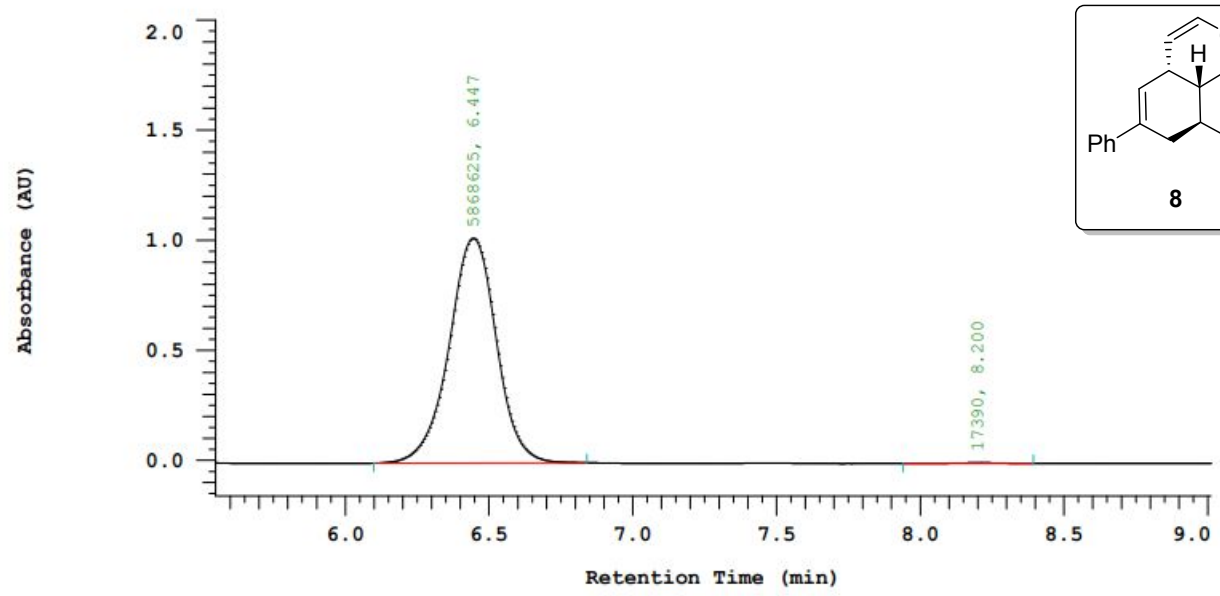

Chrom Type: Fixed WL Chromatogram, $205 \mathrm{~nm}$

Peak Quantitation: AREA

Calculation Method: AREA

\begin{tabular}{|c|c|c|c|c|}
\hline No. & $\mathrm{RT}$ & Area & Area 음 & $\mathrm{BC}$ \\
\hline 1 & 6.447 & 5868625 & 99.705 & BB \\
\hline 2 & 8.200 & 17390 & 0.295 & BB \\
\hline & & 5886015 & 100.000 & \\
\hline
\end{tabular}


The ${ }^{1} \mathrm{H}$ NMR spectrum of $9\left(400 \mathrm{MHz}, \mathrm{CDCl}_{3}\right)$

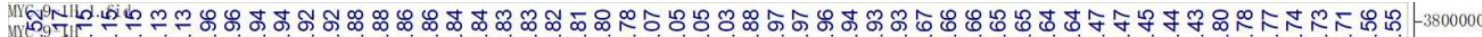

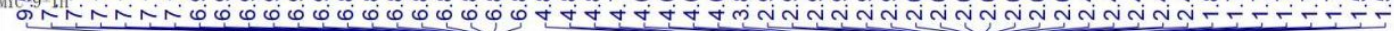
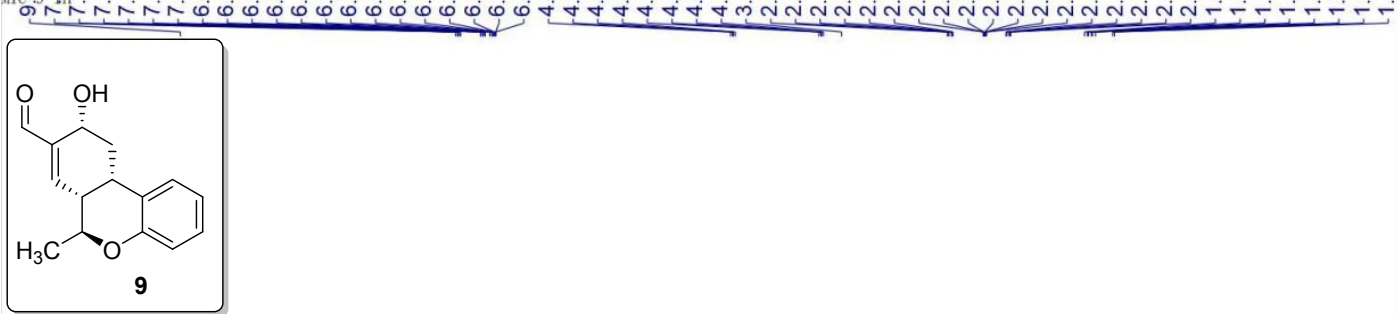

\section{0}

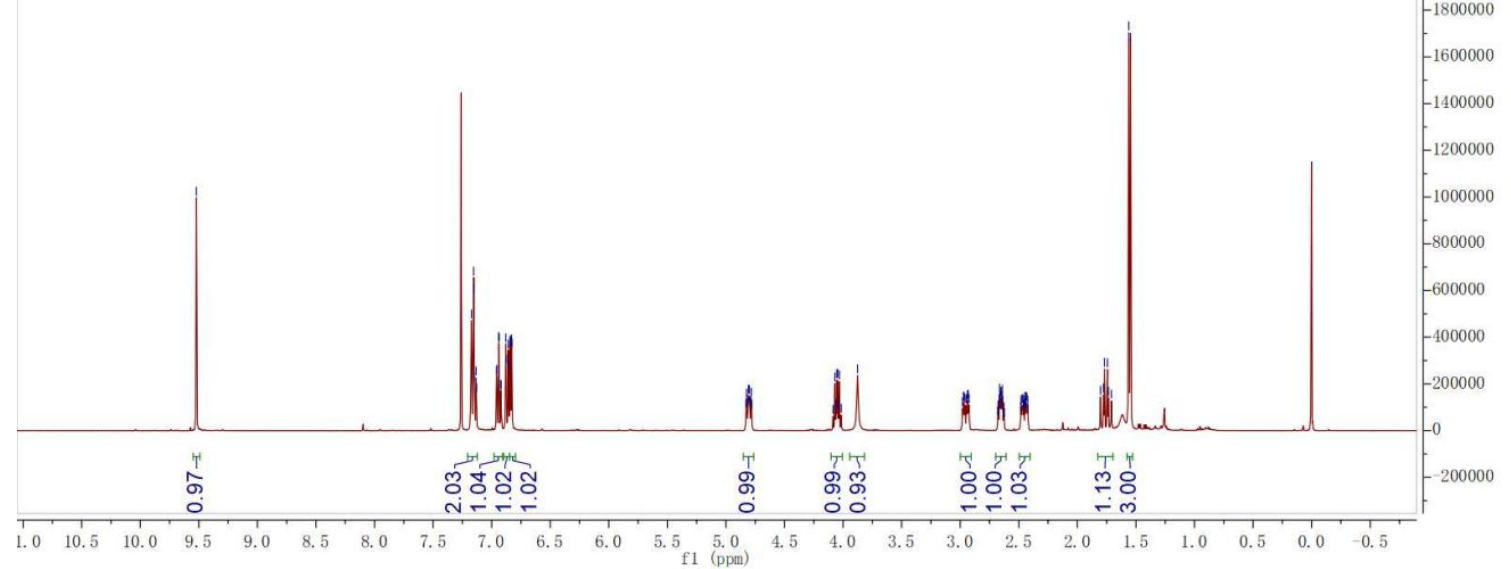

The ${ }^{13} \mathrm{C}$ NMR spectrum of $9\left(100 \mathrm{MHz}, \mathrm{CDCl}_{3}\right)$

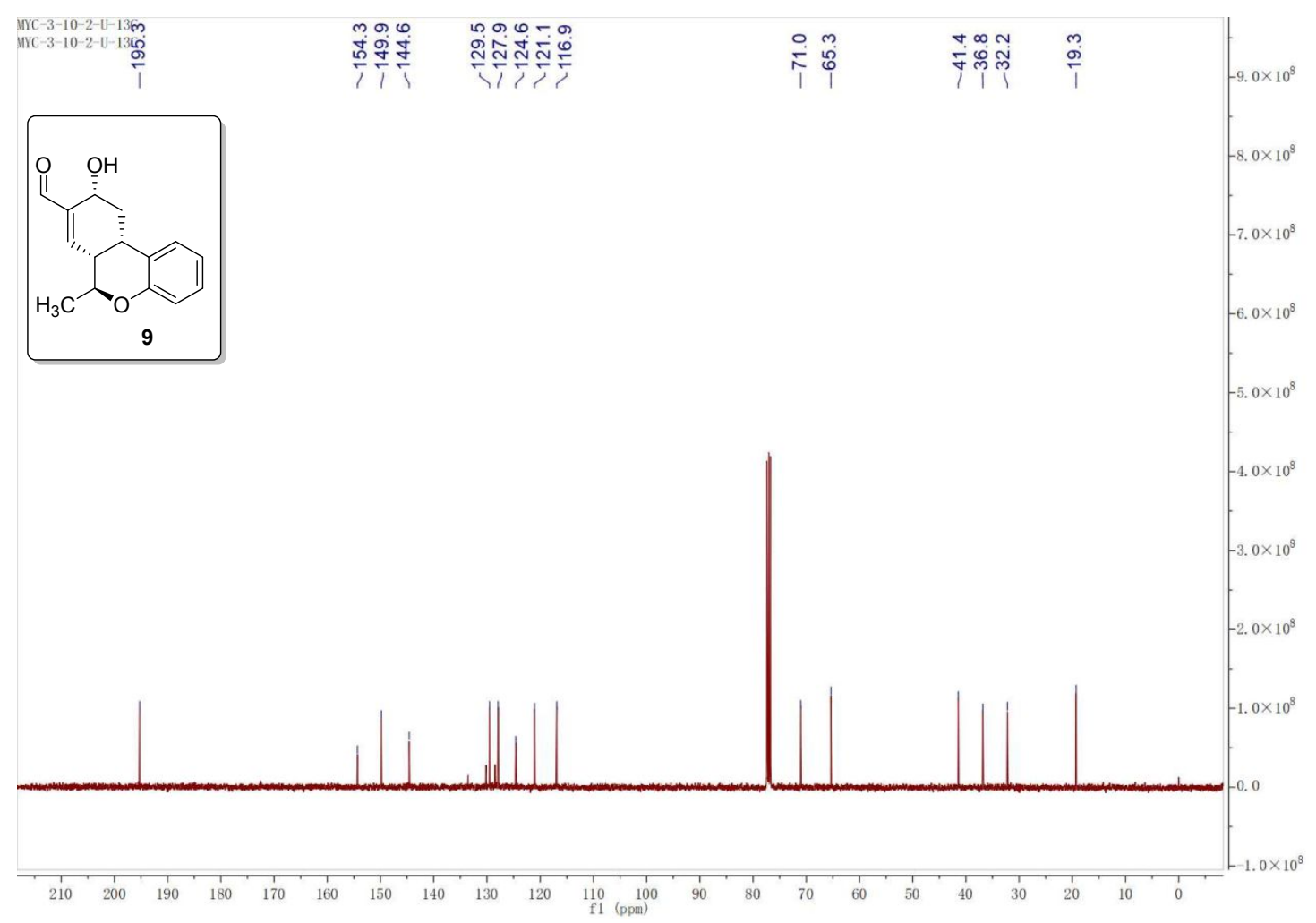




\section{The HPLC of racemic 9}

Chrom Type: Fixed WL Chromatogram, $220 \mathrm{~nm}$

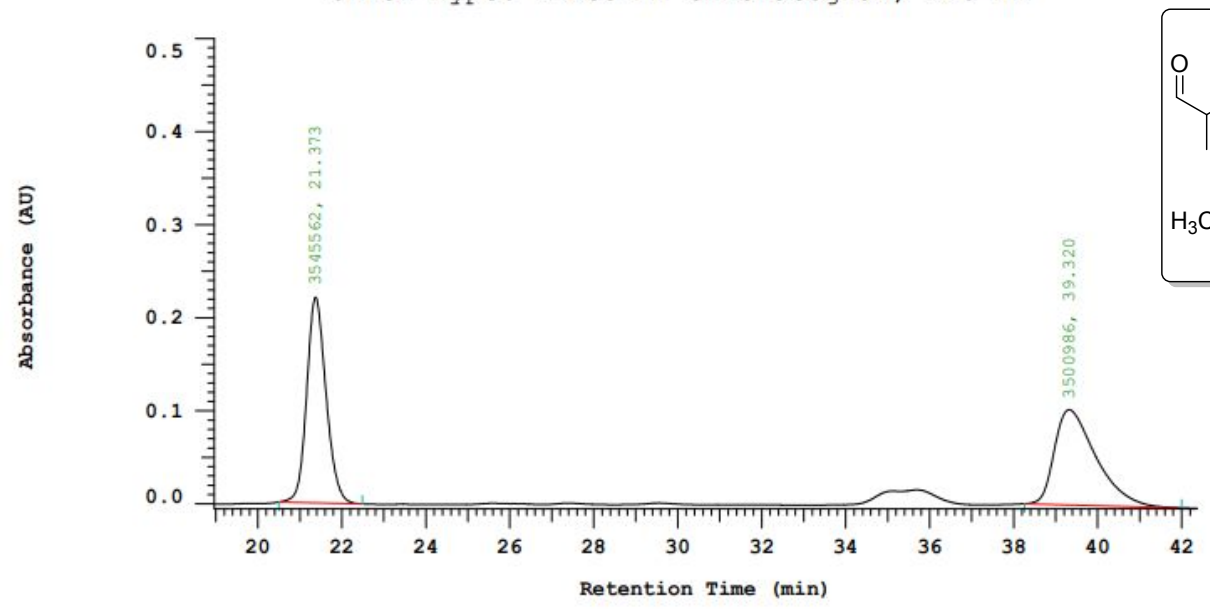

Peak Quantitation: AREA

Calculation Method: AREA응

\begin{tabular}{ccccc} 
No. & RT & Area & Area & BC \\
\hline 1 & 21.373 & 3545562 & 50.316 & BB \\
2 & 39.320 & 3500986 & 49.684 & BB \\
\hline & & 7046548 & 100.000 & \\
\hline
\end{tabular}

\section{The HPLC of chiral 9}

Chrom Type: Fixed WL Chromatogram, $220 \mathrm{~nm}$

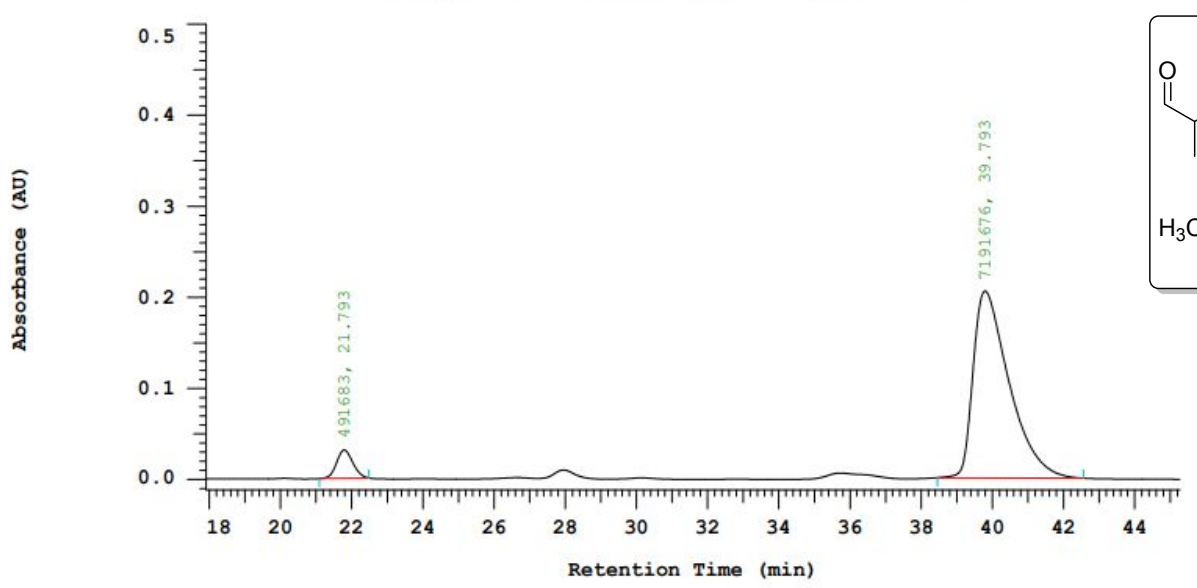

Chrom Type: Fixed WL Chromatogram, $220 \mathrm{~nm}$ Peak Quantitation: AREA

Calculation Method: AREA\&

\begin{tabular}{ccccc} 
No. & RT & Area & Area & BC \\
\hline 1 & 21.793 & 491683 & 6.399 & BB \\
2 & 39.793 & 7191676 & 93.601 & BB \\
\hline & & 7683359 & 100.000 & \\
\hline
\end{tabular}


The ${ }^{1} \mathrm{H}$ NMR spectrum of $10\left(400 \mathrm{MHz}, \mathrm{CDCl}_{3}\right)$

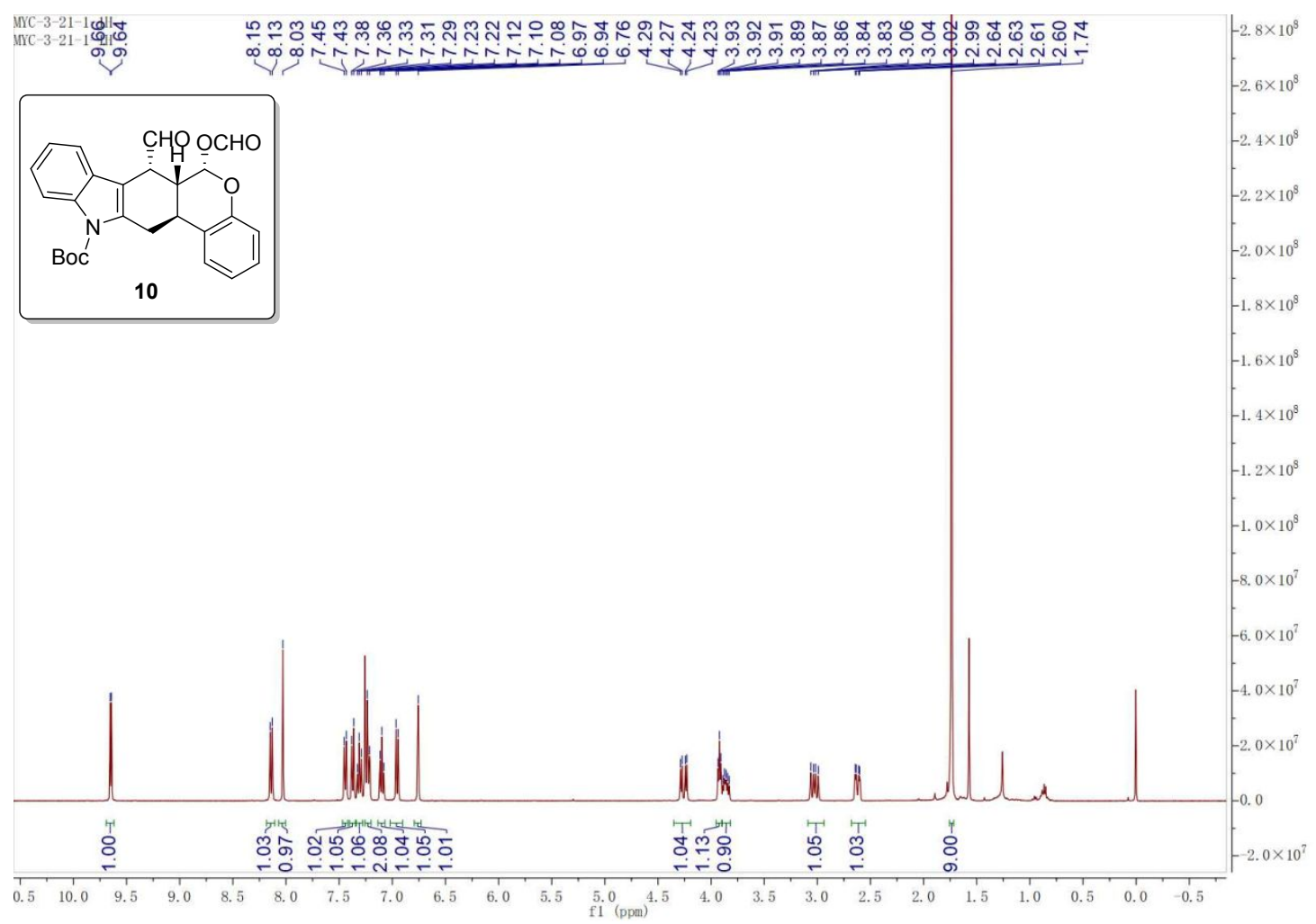

The ${ }^{13} \mathrm{C}$ NMR spectrum of $10\left(100 \mathrm{MHz}, \mathrm{CDCl}_{3}\right)$

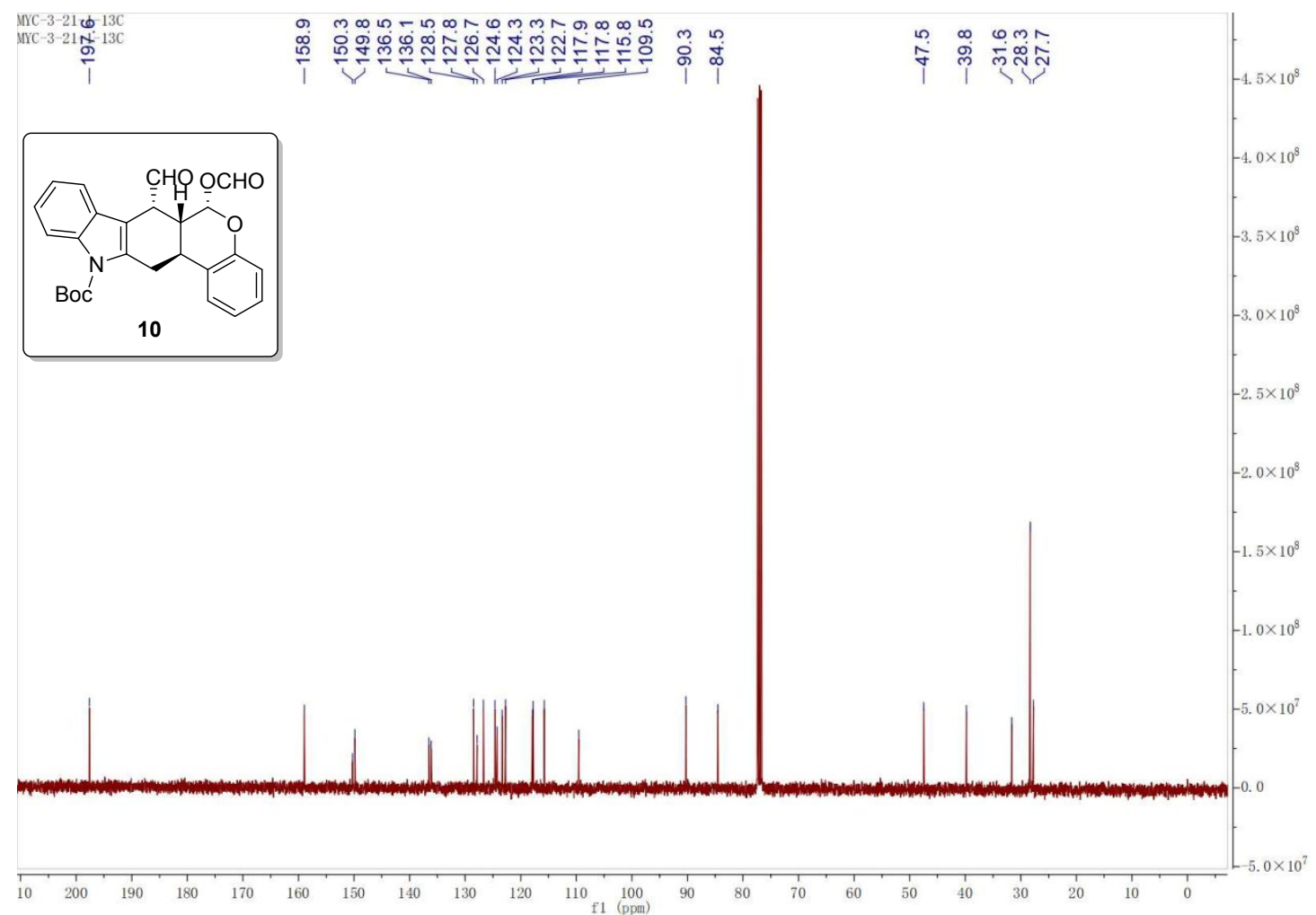




\section{The HPLC of racemic 10}

Chrom Type: Fixed WL Chromatogram, 205 nm

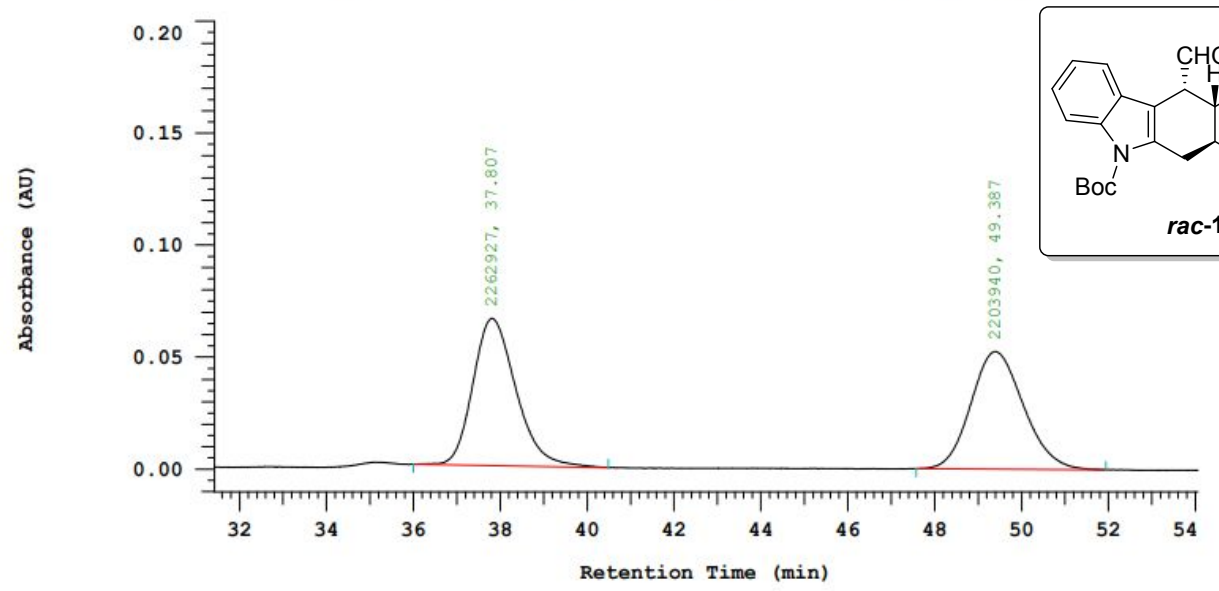

Chrom Type: Fixed WL Chromatogram, 205 nm

Peak Quantitation: AREA

Calculation Method: AREA용

\begin{tabular}{ccccc} 
No. & RT & Area & Area s & BC \\
\hline 1 & 37.807 & 2262927 & 50.660 & BB \\
2 & 49.387 & 2203940 & 49.340 & BB \\
\hline & & 4466867 & 100.000 & \\
\hline
\end{tabular}

\section{The HPLC of chiral 10}

Chrom Type: Fixed WL Chromatogram, 205 nm

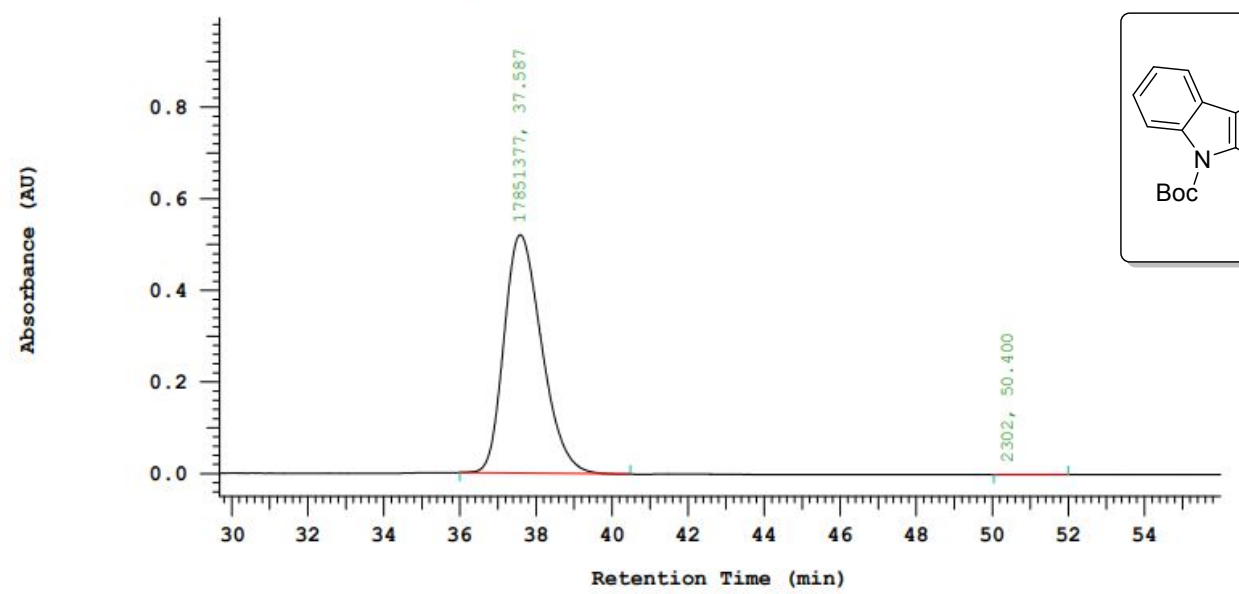

Chrom Type: Fixed WL Chromatogram, 205 nm Peak Quantitation: AREA

Calculation Method: AREA\&

\begin{tabular}{rrrrr} 
No. & RT & Area & Area $\%$ & BC \\
\hline 1 & 37.587 & 17851377 & 99.987 & BB \\
2 & 50.400 & 2302 & 0.013 & BB \\
\hline & & 17853679 & 100.000
\end{tabular}


The ${ }^{1} \mathrm{H}$ NMR spectrum of $11\left(500 \mathrm{MHz}, \mathrm{CDCl}_{3}\right)$

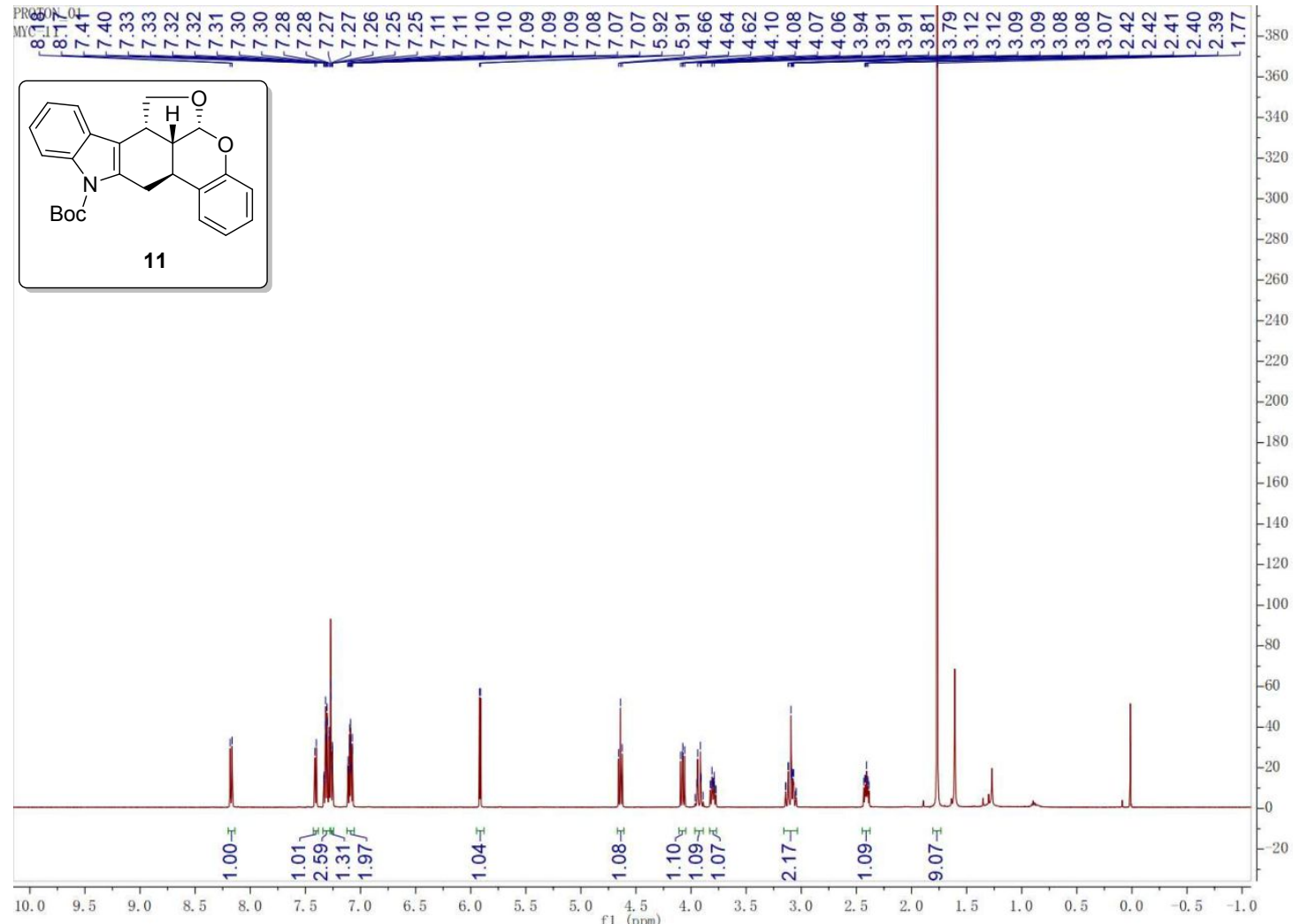

The ${ }^{13} \mathrm{C}$ NMR spectrum of $11\left(125 \mathrm{MHz}, \mathrm{CDCl}_{3}\right)$

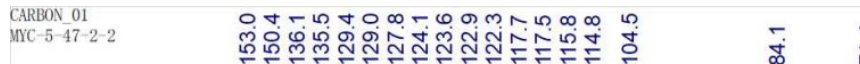

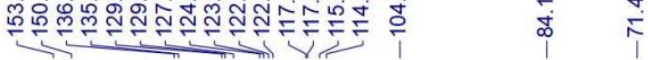
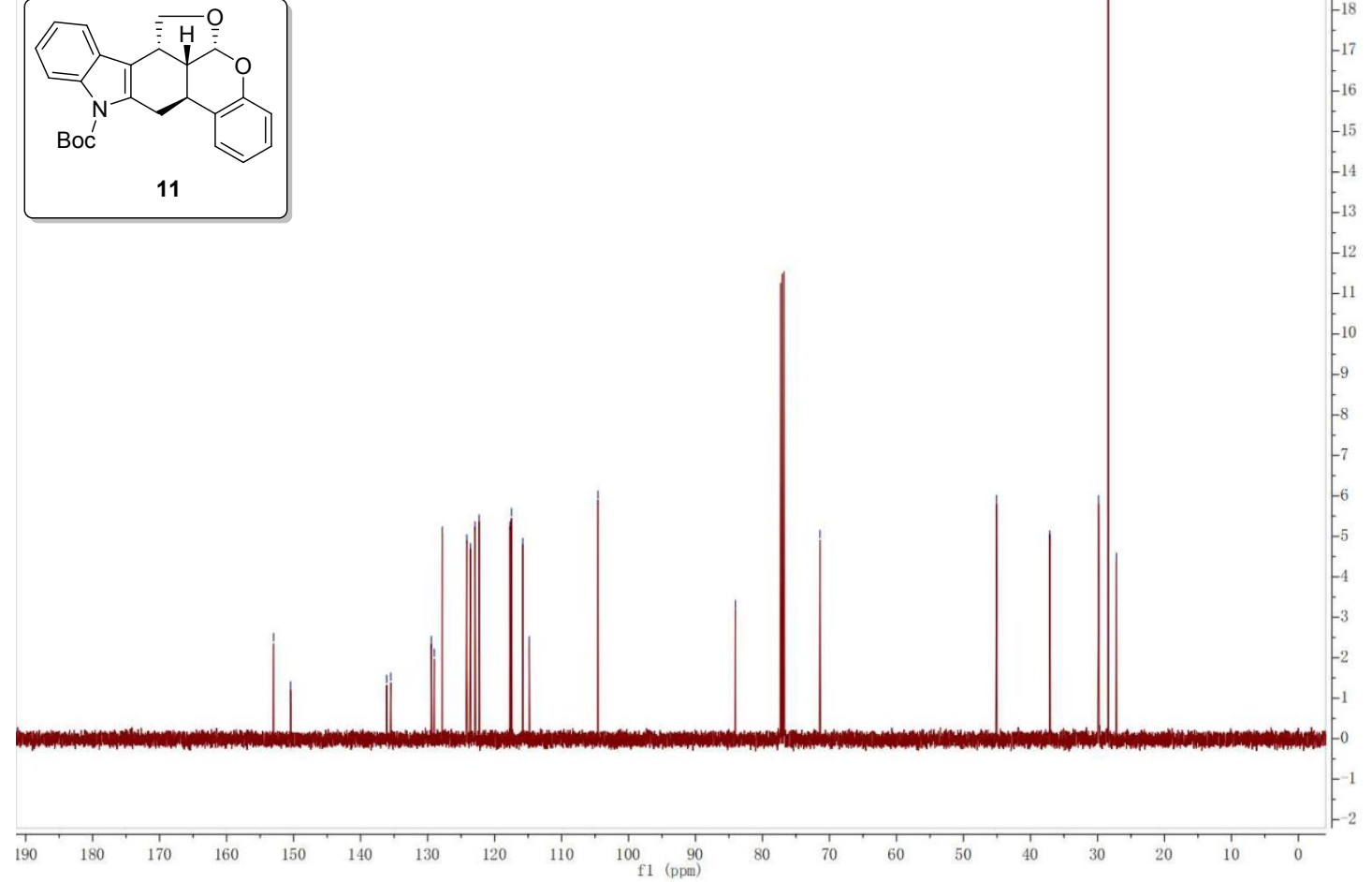
The NOSEY spectrum of $11\left(500 \mathrm{MHz}, \mathrm{CDCl}_{3}\right)$

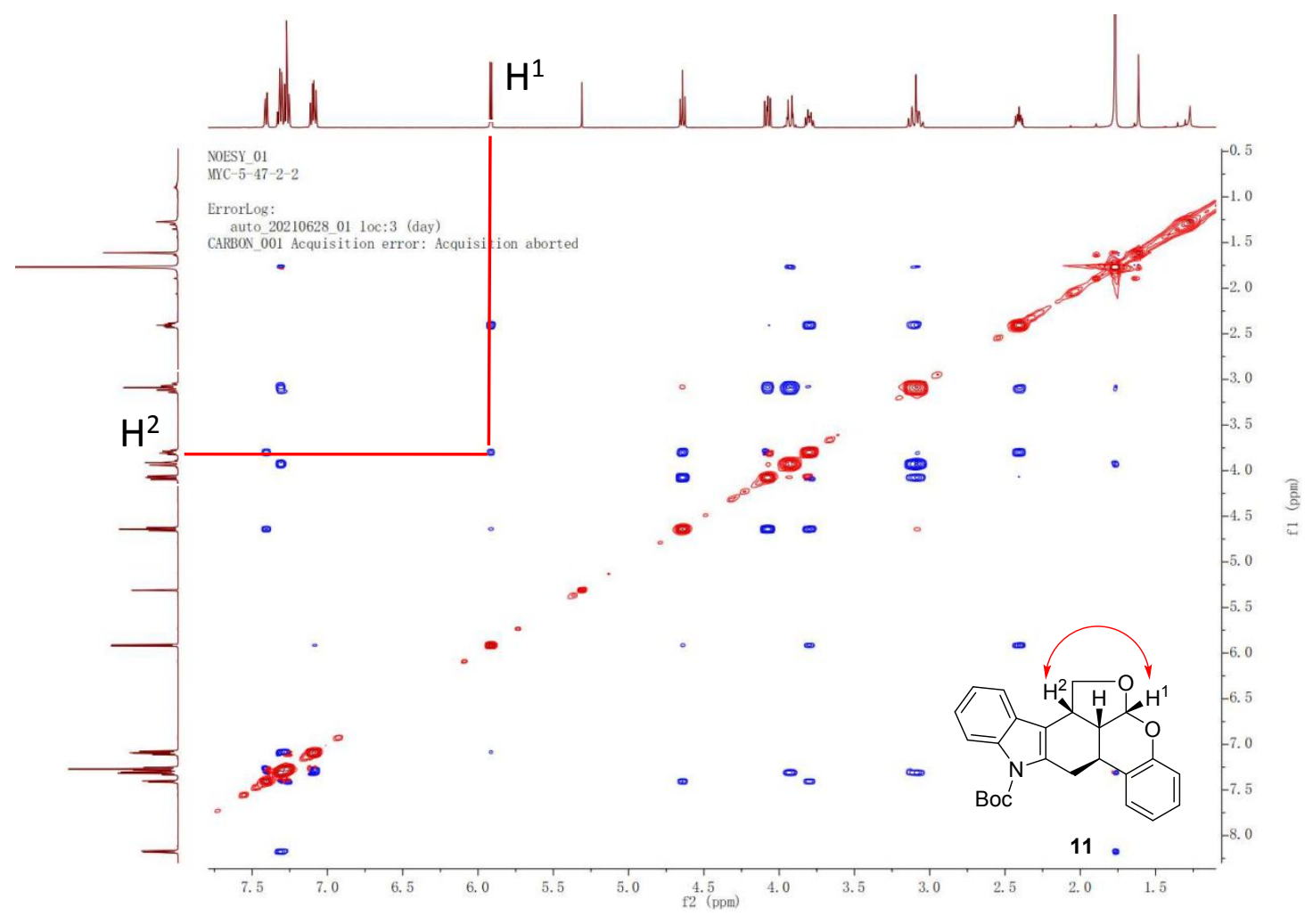




\section{The HPLC of racemic 11}

Chrom Type: Fixed WL Chromatogram, $225 \mathrm{~nm}$

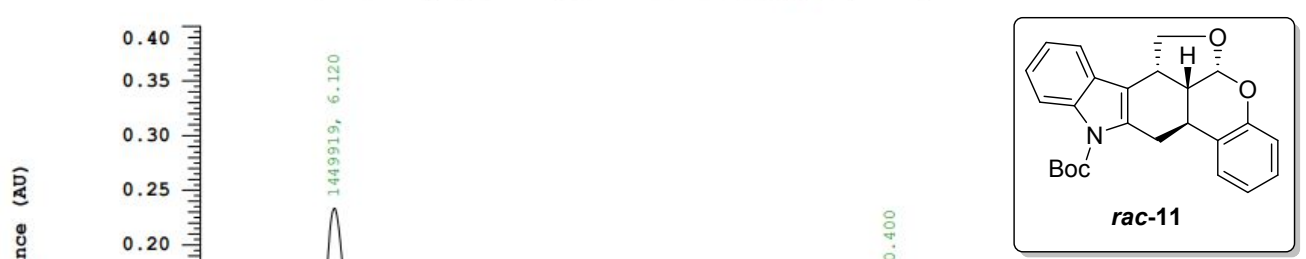

ntitation: AREA

Calculation Method: AREA응

\begin{tabular}{|c|c|c|c|c|}
\hline No. & $\mathrm{RT}$ & Area & Area $\frac{8}{6}$ & $\mathrm{BC}$ \\
\hline 1 & 6.120 & 1449919 & 50.044 & $\mathrm{BB}$ \\
\hline 2 & 10.400 & 1447393 & 49.956 & $\mathrm{BB}$ \\
\hline & & 2897312 & 100.000 & \\
\hline
\end{tabular}

\section{The HPLC of chiral 11}

Chrom Type: Fixed WL Chromatogram, $225 \mathrm{~nm}$
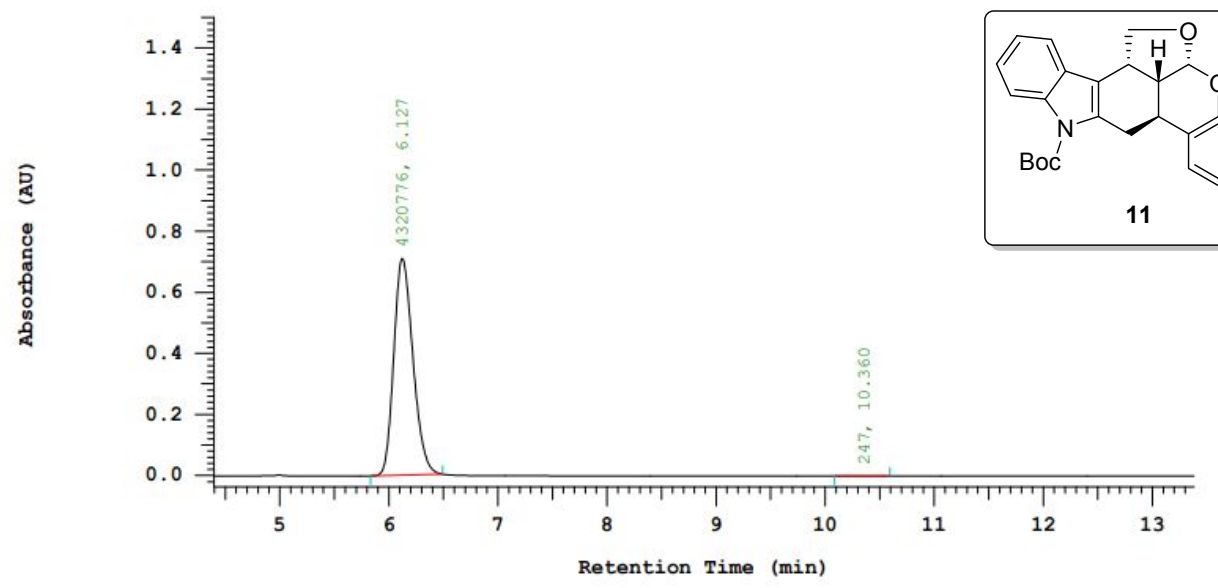

Chrom Type: Fixed WL Chromatogram, $225 \mathrm{~nm}$

Peak Quantitation: AREA

Calculation Method: AREA용

\begin{tabular}{|c|c|c|c|c|}
\hline No. & RT & Area & Area : & $\mathrm{BC}$ \\
\hline 1 & 6.127 & 4320776 & 99.994 & $\mathrm{BB}$ \\
\hline \multirow[t]{2}{*}{2} & 10.360 & 247 & 0.006 & $\mathrm{BB}$ \\
\hline & & 4321023 & 100.000 & \\
\hline
\end{tabular}


The ${ }^{1} \mathrm{H}$ NMR spectrum of $12\left(400 \mathrm{MHz}, \mathrm{CDCl}_{3}\right)$

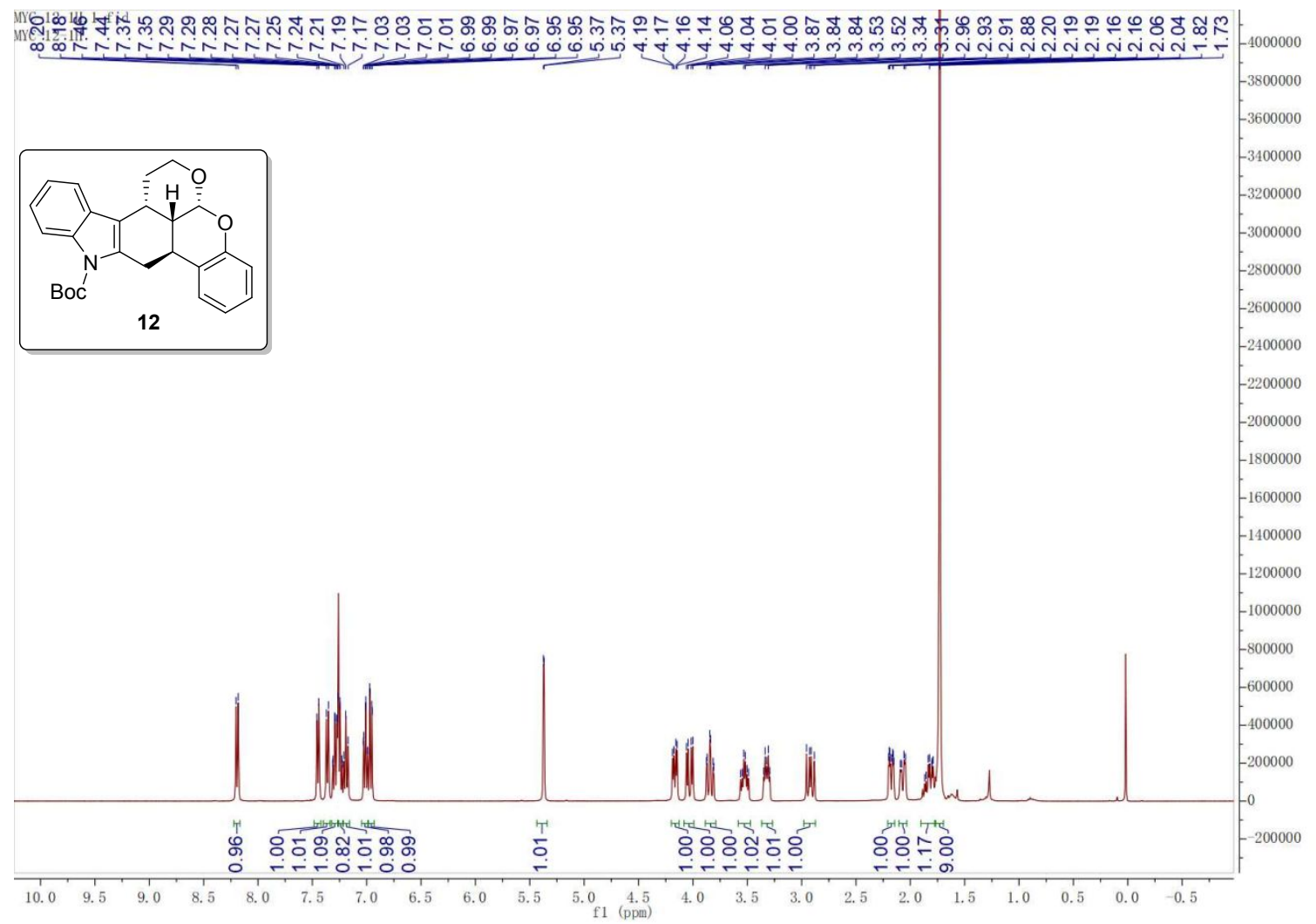

The ${ }^{13} \mathrm{C}$ NMR spectrum of $12\left(100 \mathrm{MHz}, \mathrm{CDCl}_{3}\right)$

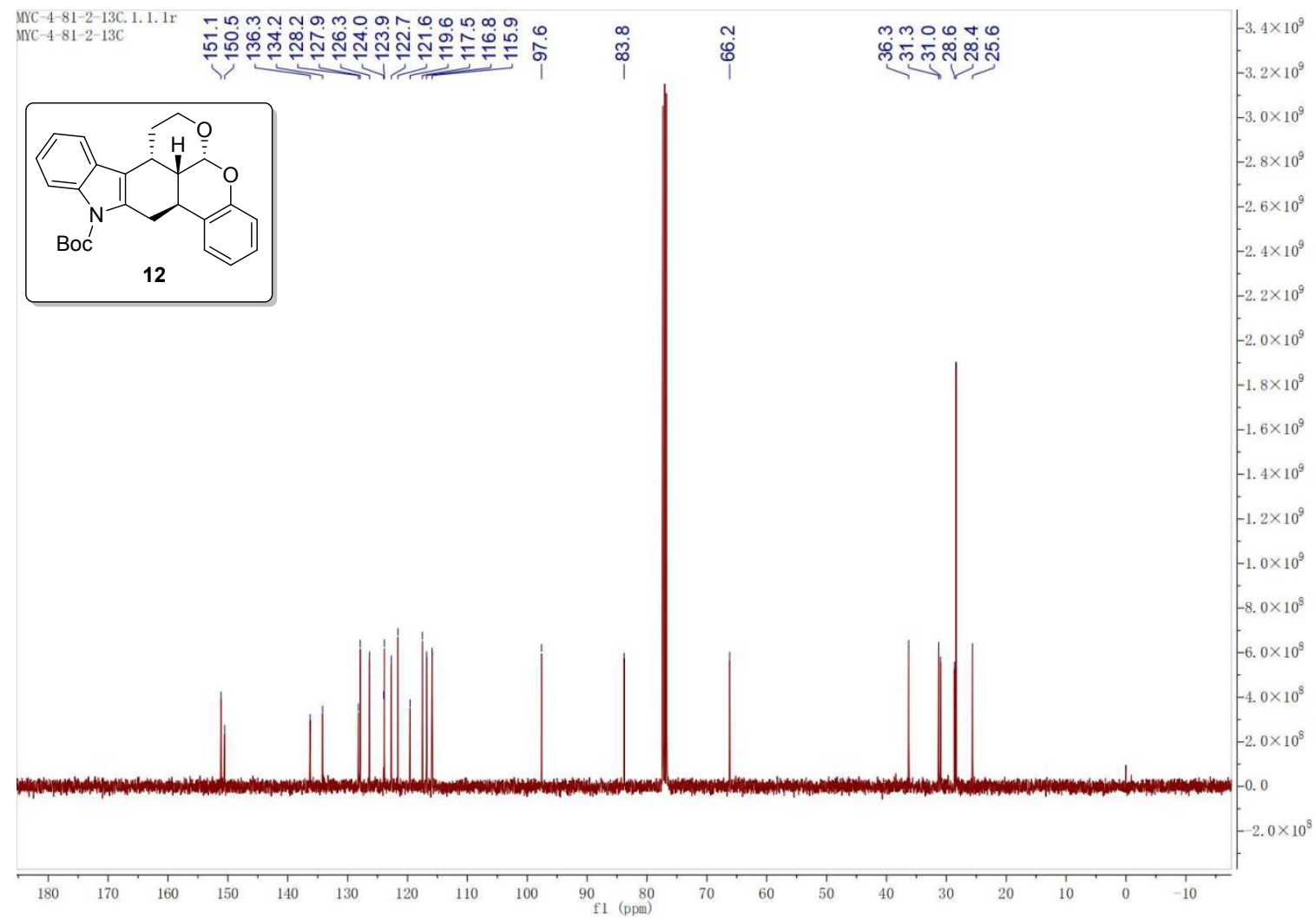




\section{The HPLC of racemic 12}

Chrom Type: Fixed WL Chromatogram, 225 nm

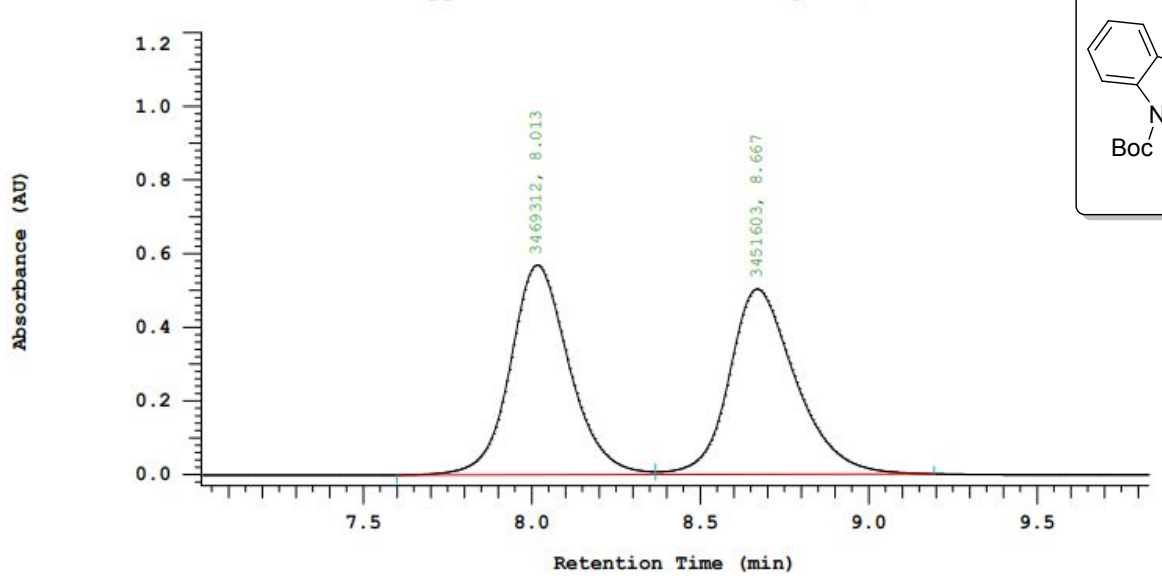

Chrom Type: Fixed WL Chromatogram, 225 nm

Peak Quantitation: AREA

Calculation Method: AREA\%

\begin{tabular}{ccccc} 
No. & RT & Area & Area & BC \\
\hline 1 & 8.013 & 3469312 & 50.128 & BV \\
2 & 8.667 & 3451603 & 49.872 & VB \\
\hline & & 6920915 & 100.000 & \\
\hline
\end{tabular}

The HPLC of chiral 12

Chrom Type: Fixed WL Chromatogram, $225 \mathrm{~nm}$

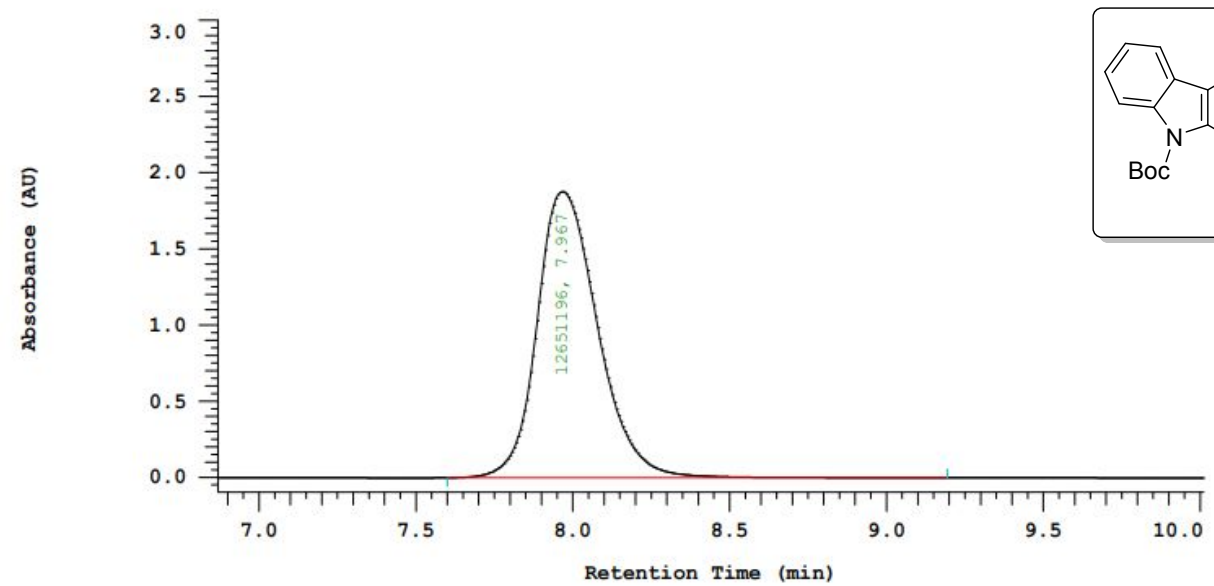

Chrom Type: Fixed WL Chromatogram, 225 nm

Peak Quantitation: AREA

Calculation Method: AREA\%

\begin{tabular}{ccccc} 
No. & RT & Area & Area \% & BC \\
\hline 1 & 7.967 & 12651196 & 100.000 & BB \\
\hline & 12651196 & 100.000 & \\
\hline
\end{tabular}


The ${ }^{1} \mathrm{H}$ NMR spectrum of $13\left(400 \mathrm{MHz}, \mathrm{CDCl}_{3}\right)$

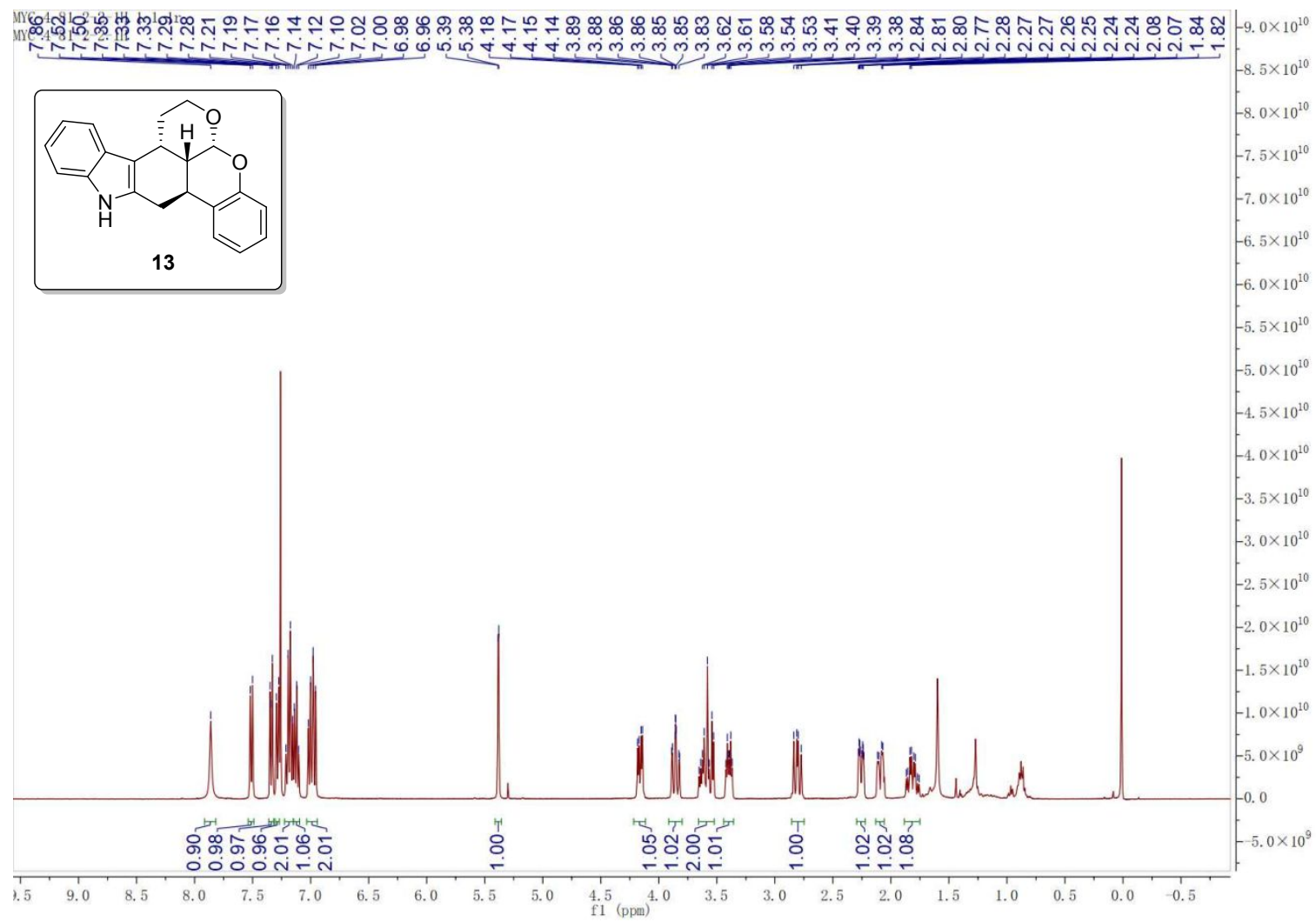

The ${ }^{13} \mathrm{C}$ NMR spectrum of $13\left(100 \mathrm{MHz}, \mathrm{CDCl}_{3}\right)$

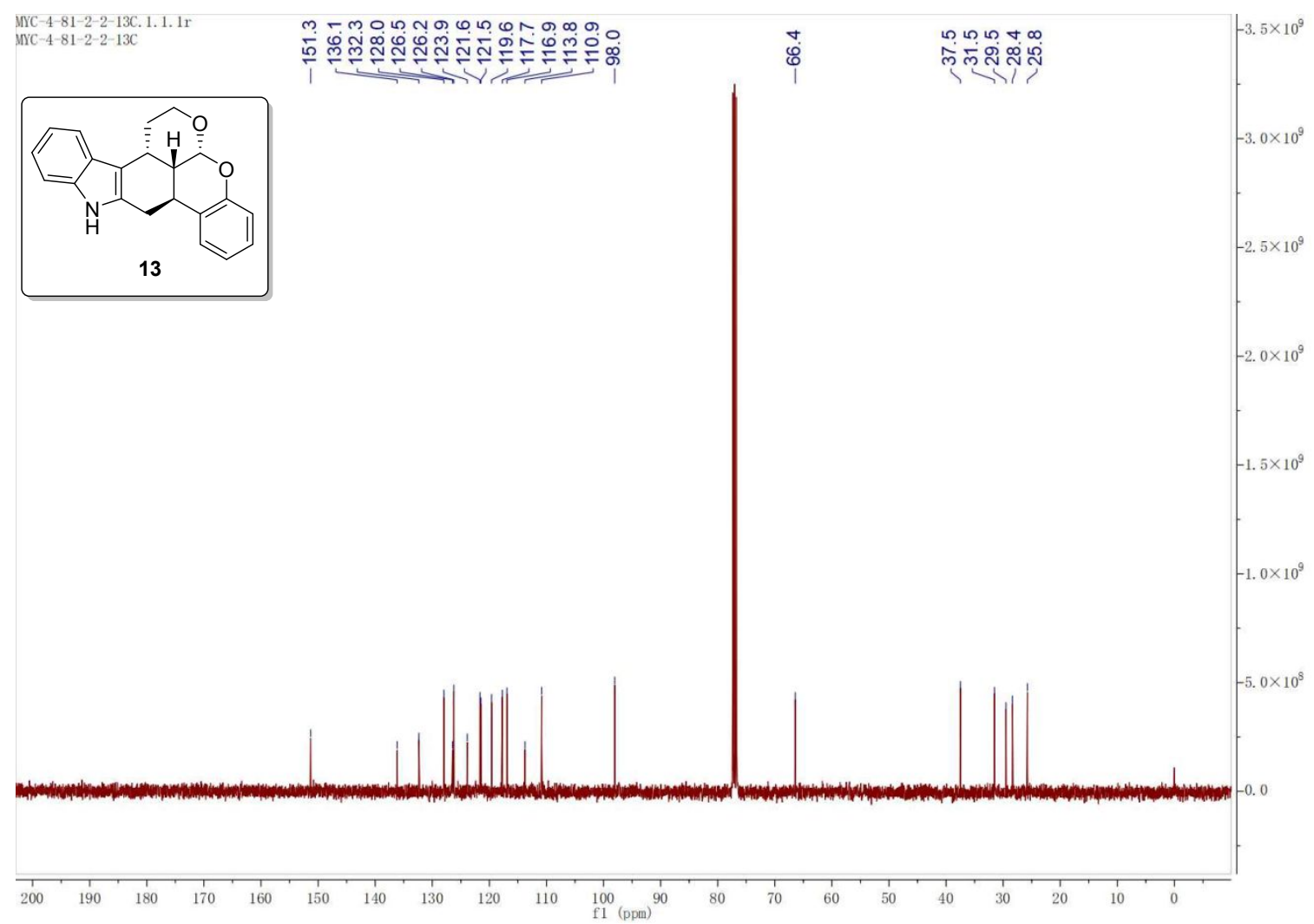




\section{The HPLC of racemic 13}

Chrom Type: Fixed WL Chromatogram, $225 \mathrm{~nm}$

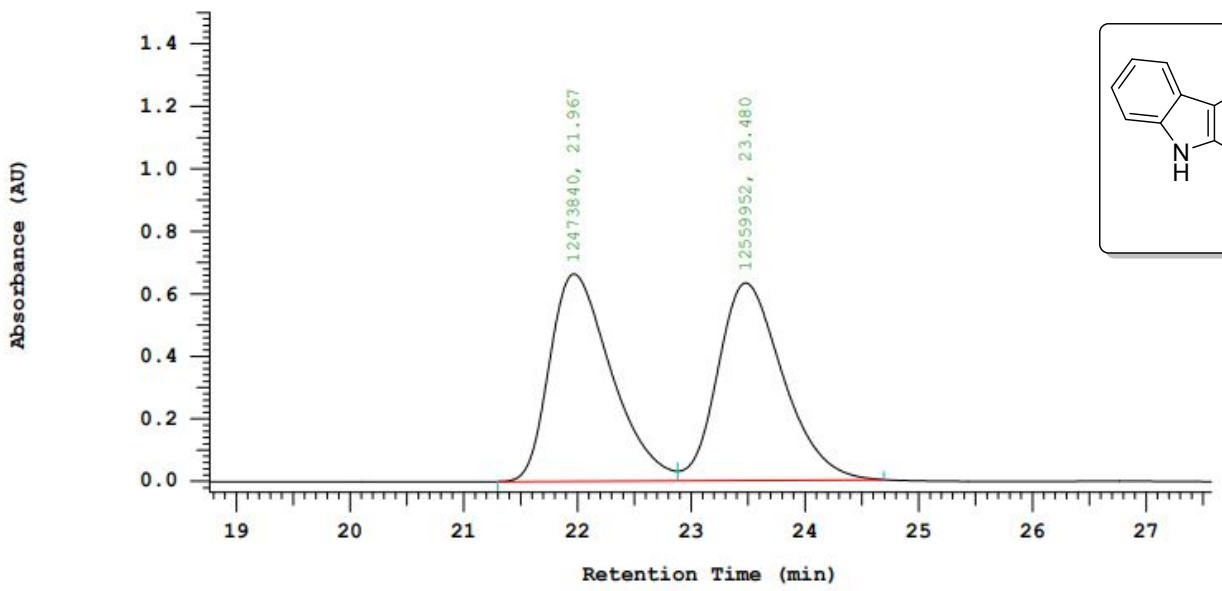

Chrom Type: Fixed WL Chromatogram, $225 \mathrm{~nm}$

Peak Quantitation: AREA

Calculation Method: AREA\%

\begin{tabular}{ccccc} 
No. & RT & Area & Area & BC \\
\hline 1 & 21.967 & 12473840 & 49.828 & BV \\
2 & 23.480 & 12559952 & 50.172 & VB \\
\hline & & 25033792 & 100.000 & \\
\hline
\end{tabular}

\section{The HPLC of chiral 13}

Chrom Type: Fixed WL Chromatogram, 225 nm

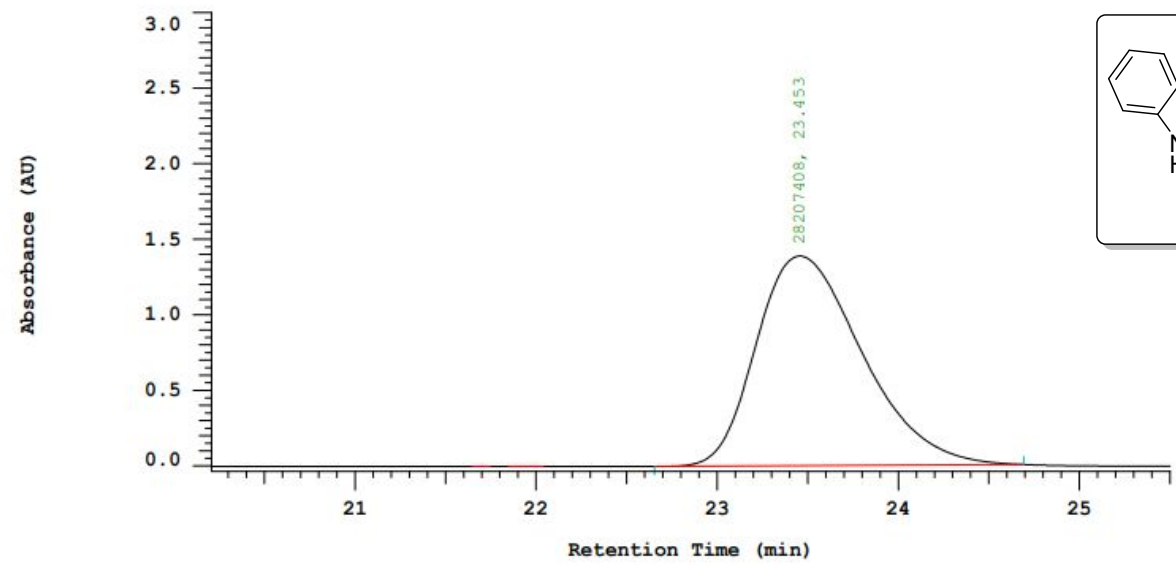

Chrom Type: Fixed WL Chromatogram, $225 \mathrm{~nm}$

Peak Quantitation: AREA

Calculation Method: AREA

\begin{tabular}{ccccc} 
No. & RT & Area & Area & BC \\
\hline 1 & 23.453 & 28207408 & 100.000 & BB \\
\hline & 28207408 & 100.000
\end{tabular}


The ${ }^{1} \mathrm{H}$ NMR spectrum of 14 (400 MHz, DMSO- $\left.d_{6}\right)$

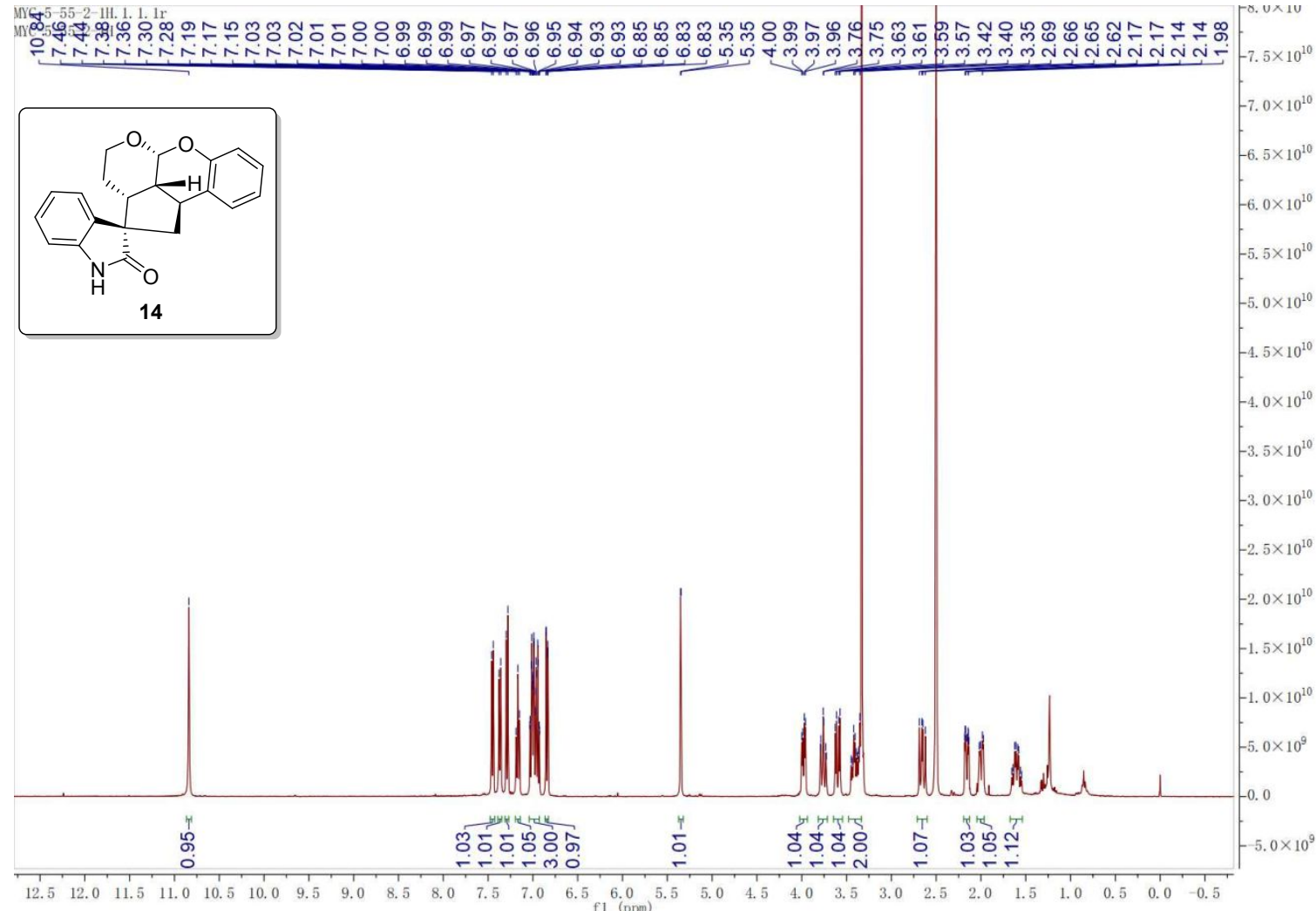

The ${ }^{13} \mathrm{C}$ NMR spectrum of 14 (100 MHz, DMSO- $\left.d_{6}\right)$

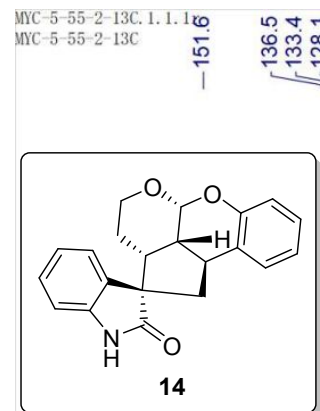




\section{The HPLC of racemic 14}

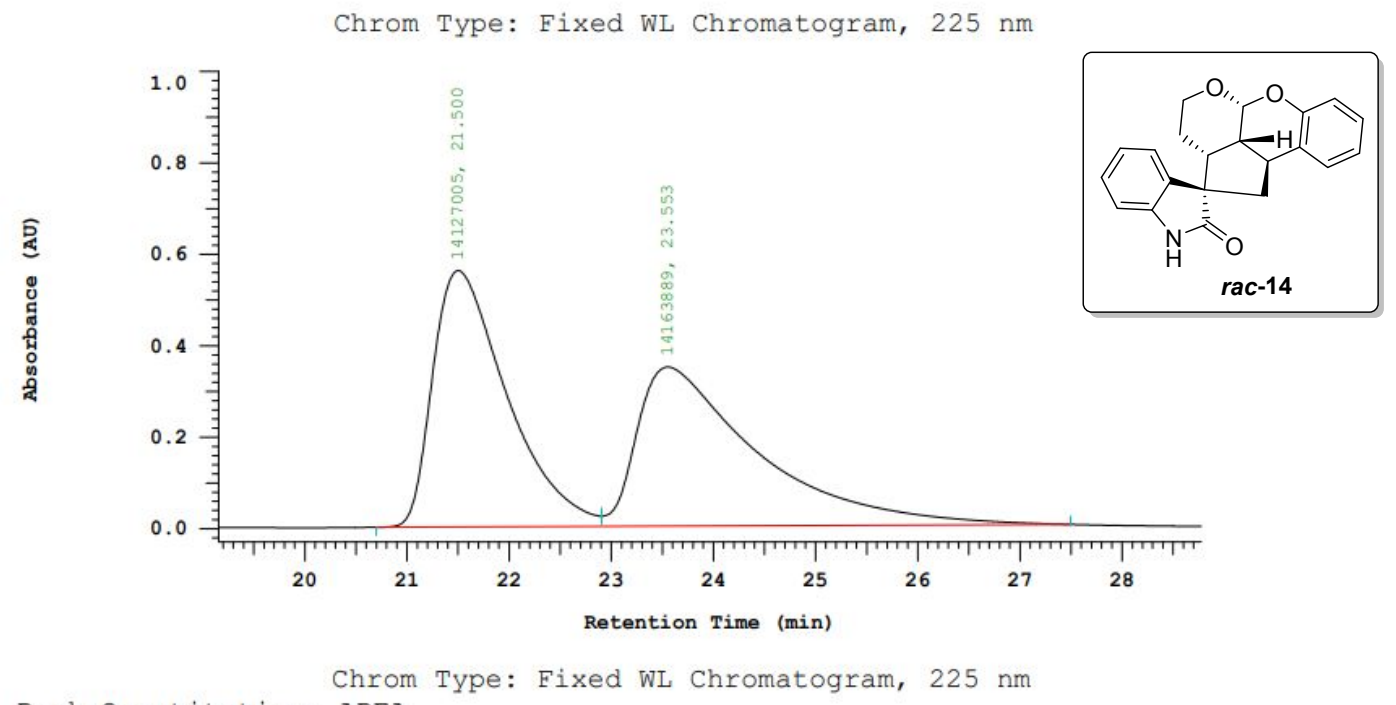

Peak Quantitation: AREA

Calculation Method: AREA\%

\begin{tabular}{ccccc} 
No. & RT & Area & Area \% & BC \\
\hline 1 & 21.500 & 14127005 & 49.935 & BV \\
2 & 23.553 & 14163889 & 50.065 & VB \\
\hline & & 28290894 & 100.000 &
\end{tabular}

The HPLC of chiral 14

Chrom Type: Fixed WL Chromatogram, $225 \mathrm{~nm}$

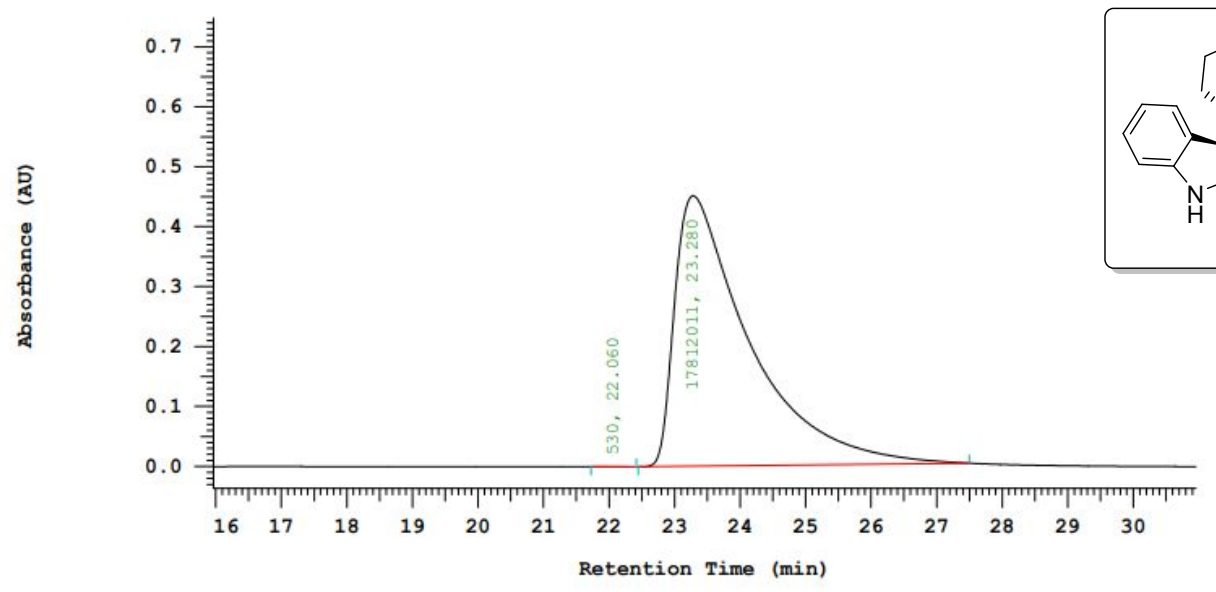

Chrom Type: Fixed WL Chromatogram, 225 nm

Peak Quantitation: AREA

Calculation Method: AREA

\begin{tabular}{|c|c|c|c|c|}
\hline No. & RT & Area & Area $\frac{\circ}{8}$ & $\mathrm{BC}$ \\
\hline 1 & 22.060 & 530 & 0.003 & BB \\
\hline 2 & 23.280 & 17812011 & 99.997 & BB \\
\hline & & 17812541 & 100.000 & \\
\hline
\end{tabular}


The ${ }^{1} \mathrm{H}$ NMR spectrum of $15\left(400 \mathrm{MHz}, \mathrm{CDCl}_{3}\right)$

MGol

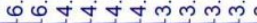
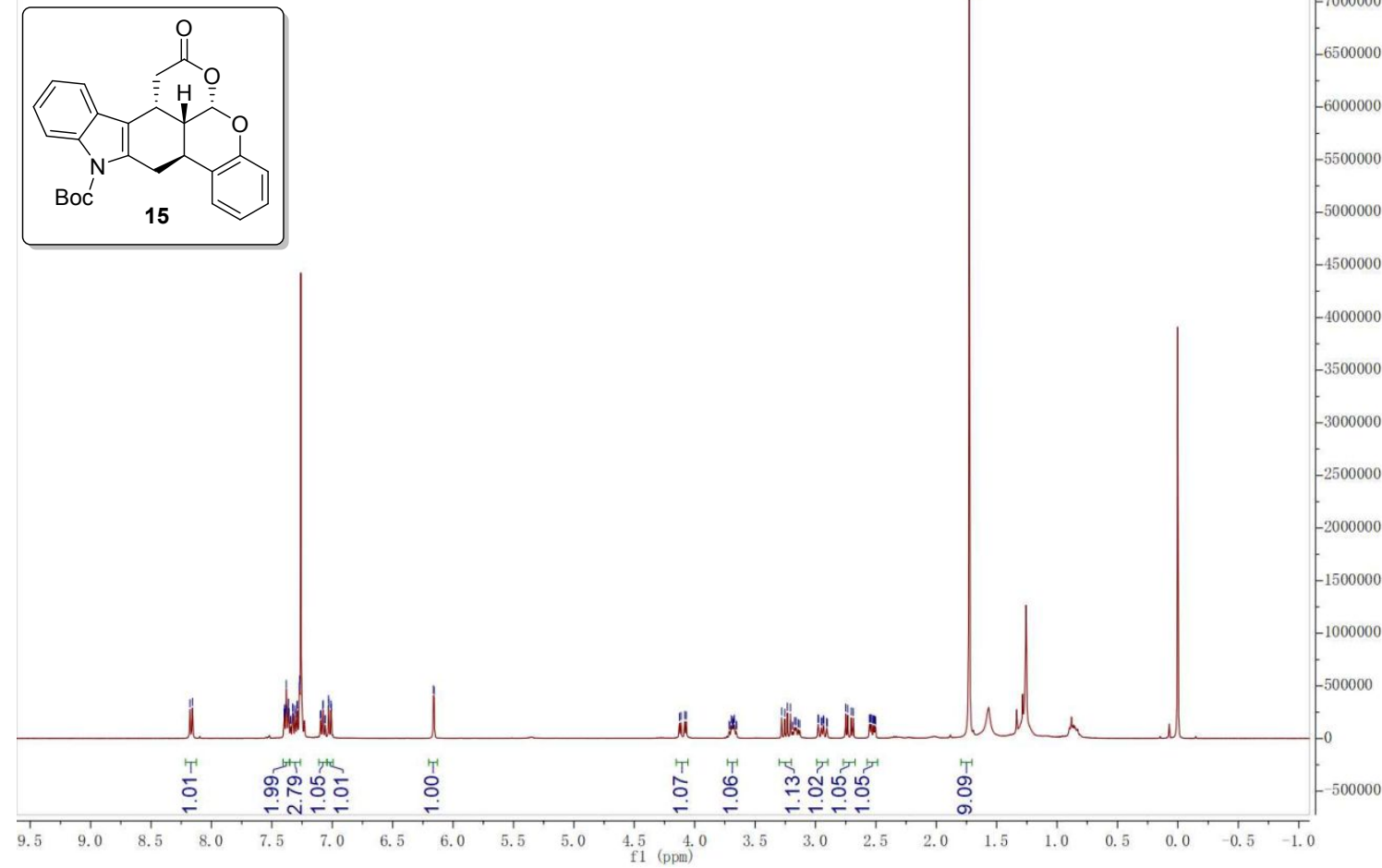

The ${ }^{13} \mathrm{C}$ NMR spectrum of $15\left(100 \mathrm{MHz}, \mathrm{CDCl}_{3}\right)$

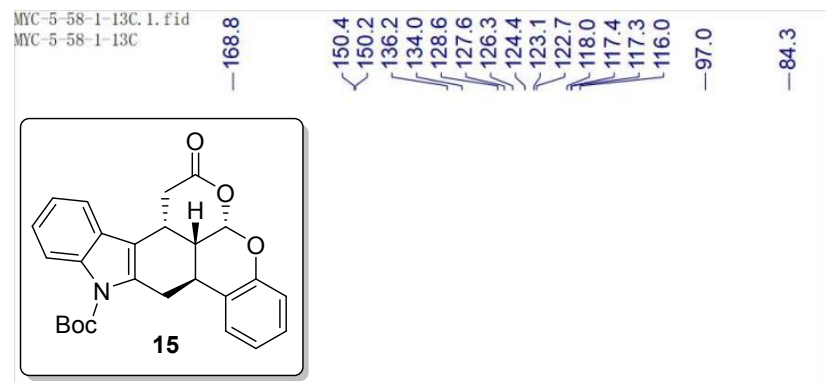

?.


The HPLC of racemic 15

Chrom Type: Fixed WL Chromatogram, 205 nm

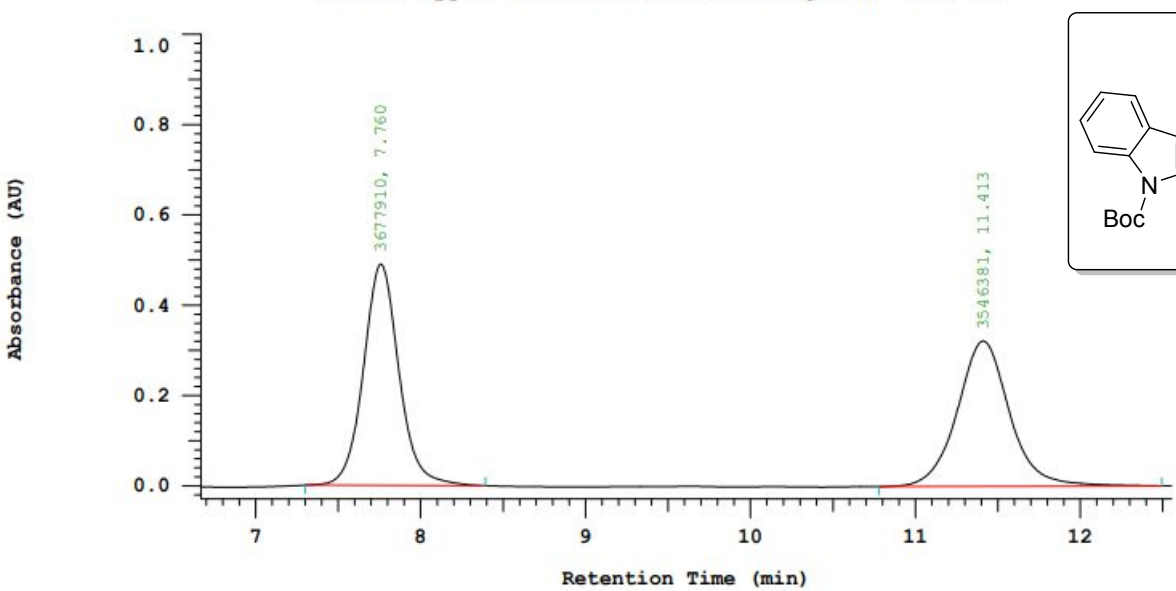

Chrom Type: Fixed WL Chromatogram, 205 nm

Peak Quantitation: AREA

Calculation Method: AREA

\begin{tabular}{rrrrr} 
No. & \multicolumn{1}{c}{ RT } & Area & Area \% & BC \\
\hline 1 & 7.760 & 3677910 & 50.910 & BB \\
2 & 11.413 & 3546381 & 49.090 & BB \\
\hline & & 7224291 & 100.000 &
\end{tabular}

\section{The HPLC of chiral 15}

Chrom Type: Fixed WL Chromatogram, 205 nm

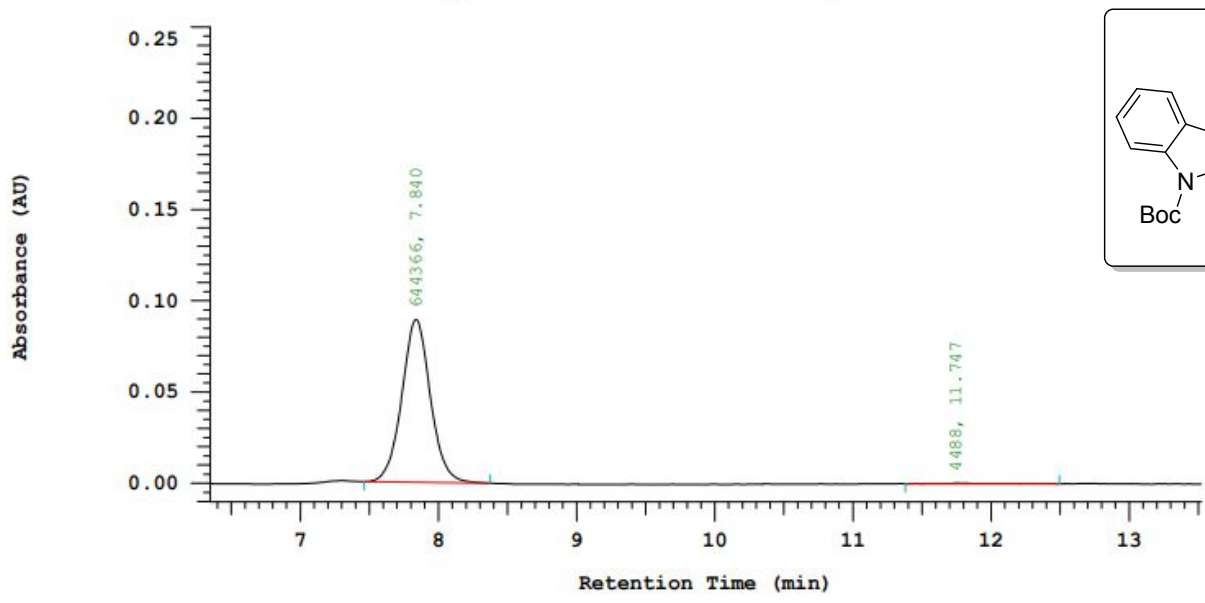

Chrom Type: Fixed WL Chromatogram, 205 nm Peak Quantitation: AREA

Calculation Method: AREA

\begin{tabular}{|c|c|c|c|c|}
\hline No. & RT & Area & Area \% & $\mathrm{BC}$ \\
\hline 1 & 7.840 & 644366 & 99.308 & $\mathrm{BB}$ \\
\hline \multirow[t]{2}{*}{2} & 11.747 & 4488 & 0.692 & $\mathrm{BB}$ \\
\hline & & 648854 & 100.000 & \\
\hline
\end{tabular}


The ${ }^{1} \mathrm{H}$ NMR spectrum of $16\left(400 \mathrm{MHz}, \mathrm{CDCl}_{3}\right)$

M603050

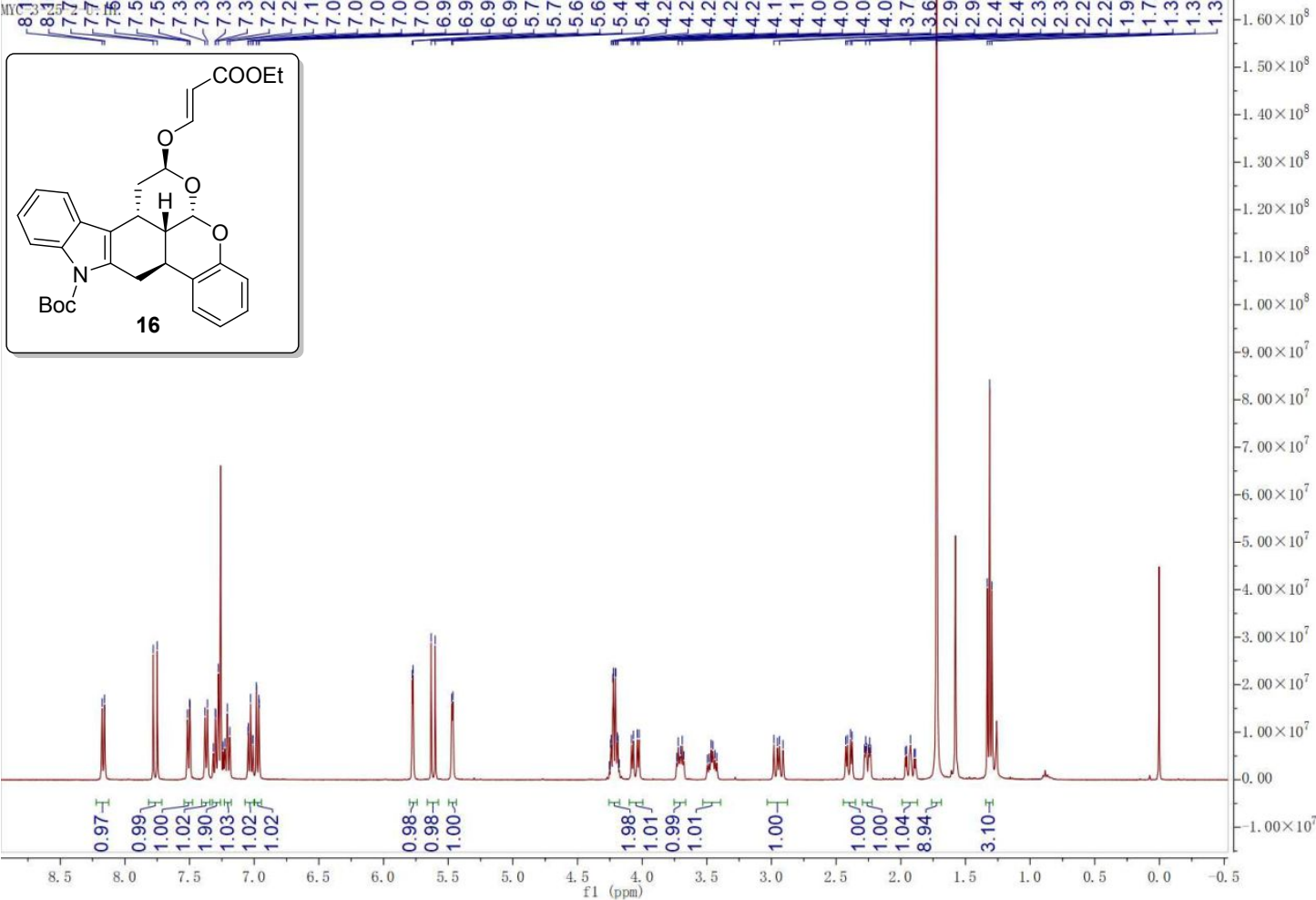

The ${ }^{13} \mathrm{C}$ NMR spectrum of $16\left(100 \mathrm{MHz}, \mathrm{CDCl}_{3}\right)$

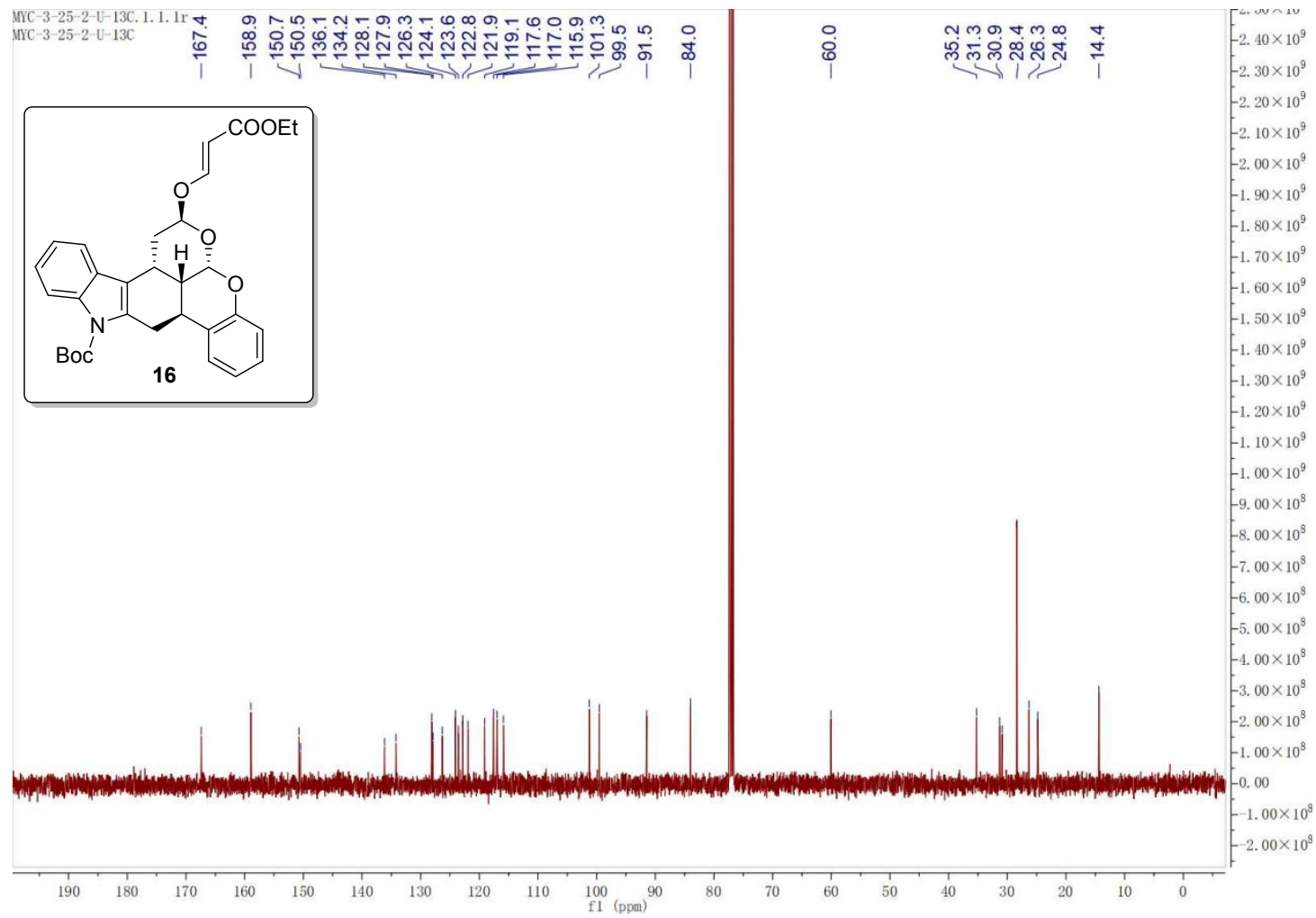


The NOSEY spectrum of $16\left(500 \mathrm{MHz}, \mathrm{CDCl}_{3}\right)$

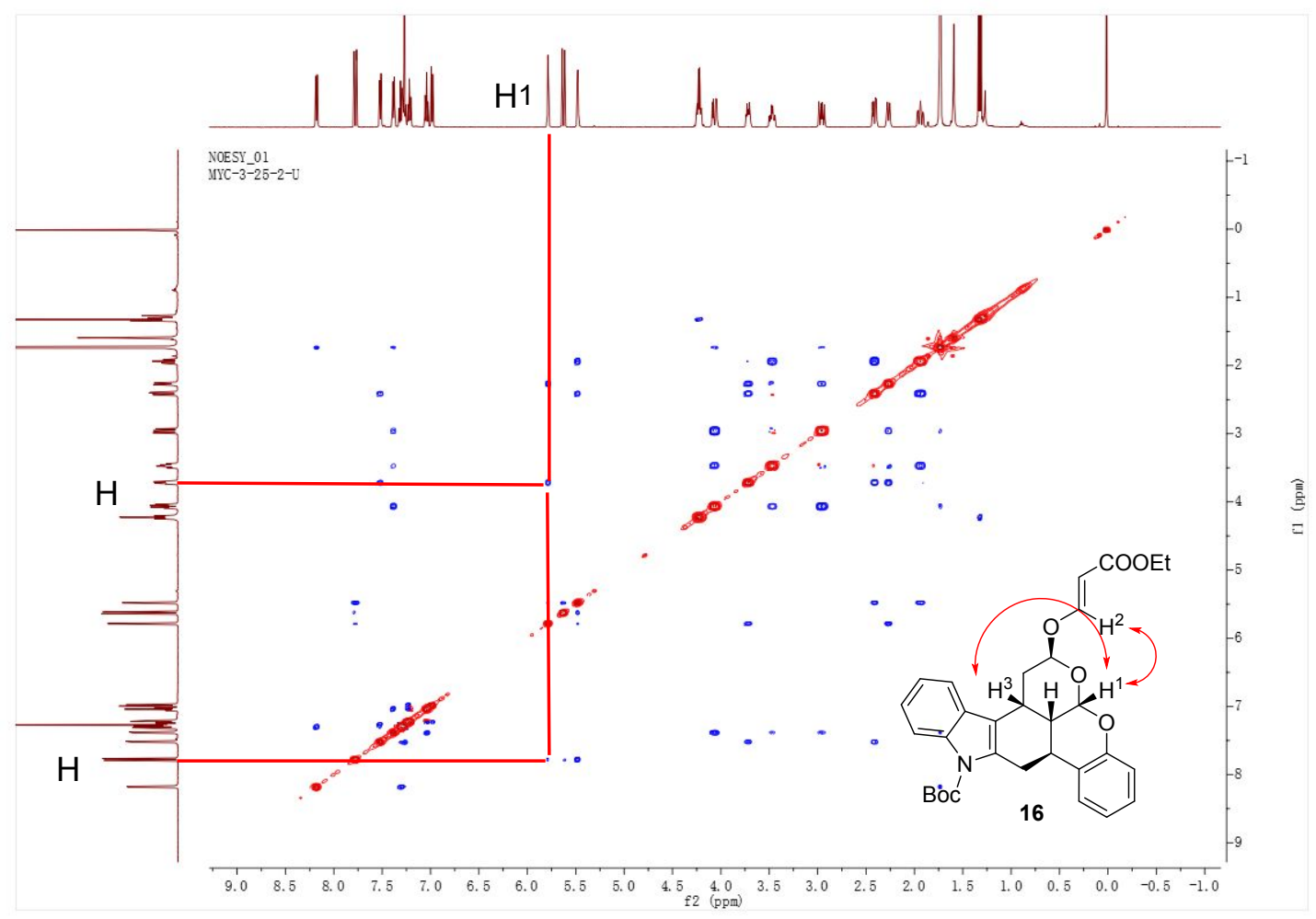

The HPLC of racemic 16

Chrom Type: Fixed WL Chromatogram, 225 nm

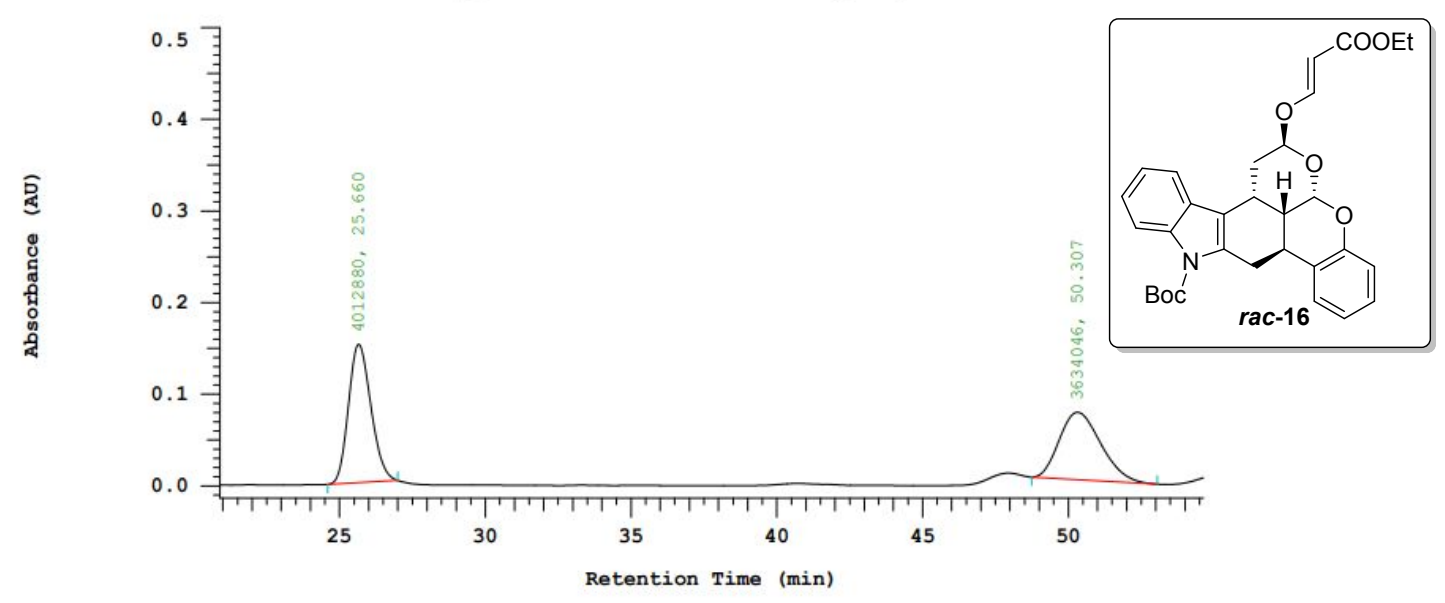

Chrom Type: Fixed WL Chromatogram, $225 \mathrm{~nm}$

Peak Quantitation: AREA

Calculation Method: AREA용

\begin{tabular}{ccccc} 
No. & RT & Area & Area & BC \\
\hline 1 & 25.660 & 4012880 & 52.477 & BB \\
2 & 50.307 & 3634046 & 47.523 & BB \\
\hline & & 7646926 & 100.000 & \\
\hline
\end{tabular}




\section{The HPLC of chiral 16}

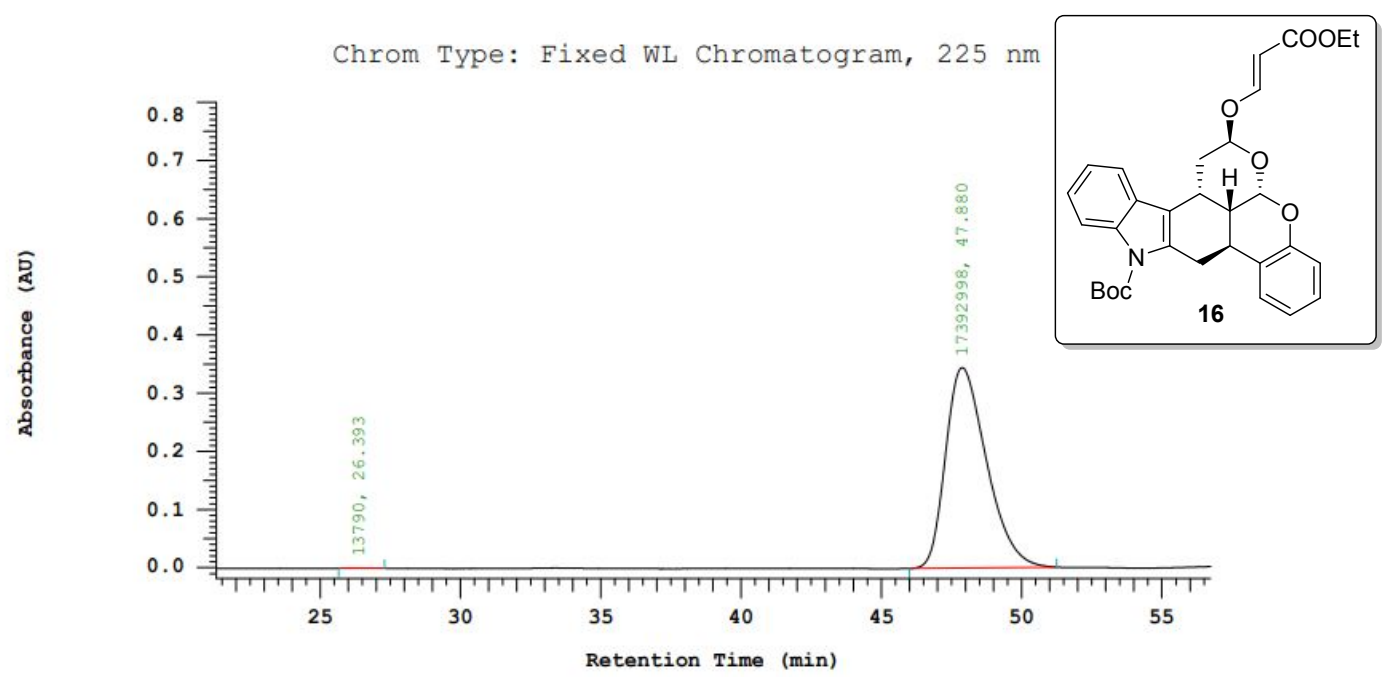

Chrom Type: Fixed WL Chromatogram, $225 \mathrm{~nm}$

Peak Quantitation: AREA

Calculation Method: AREA\%

\begin{tabular}{crrrr} 
No. & RT & Area & Area & B \\
\hline 1 & 26.393 & 13790 & 0.079 & BB \\
2 & 47.880 & 17392998 & 99.921 & BB \\
\hline & & 17406788 & 100.000 & \\
\hline
\end{tabular}

\section{The ${ }^{1} \mathrm{H}$ NMR spectrum of $16^{\prime}\left(500 \mathrm{MHz}, \mathrm{CDCl}_{3}\right)$}

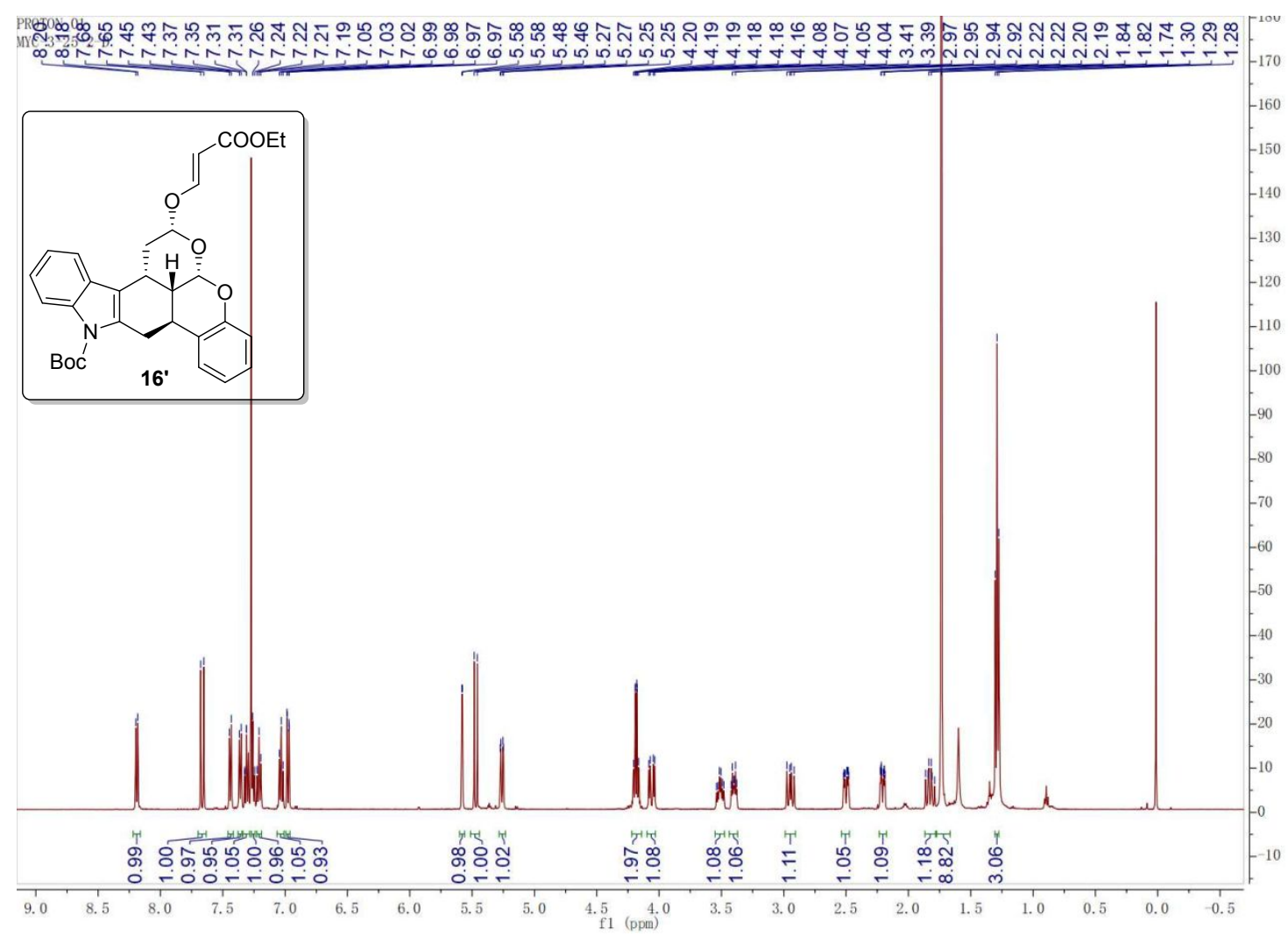


The ${ }^{13} \mathrm{C}$ NMR spectrum of $16^{\prime}\left(100 \mathrm{MHz}, \mathrm{CDCl}_{3}\right)$

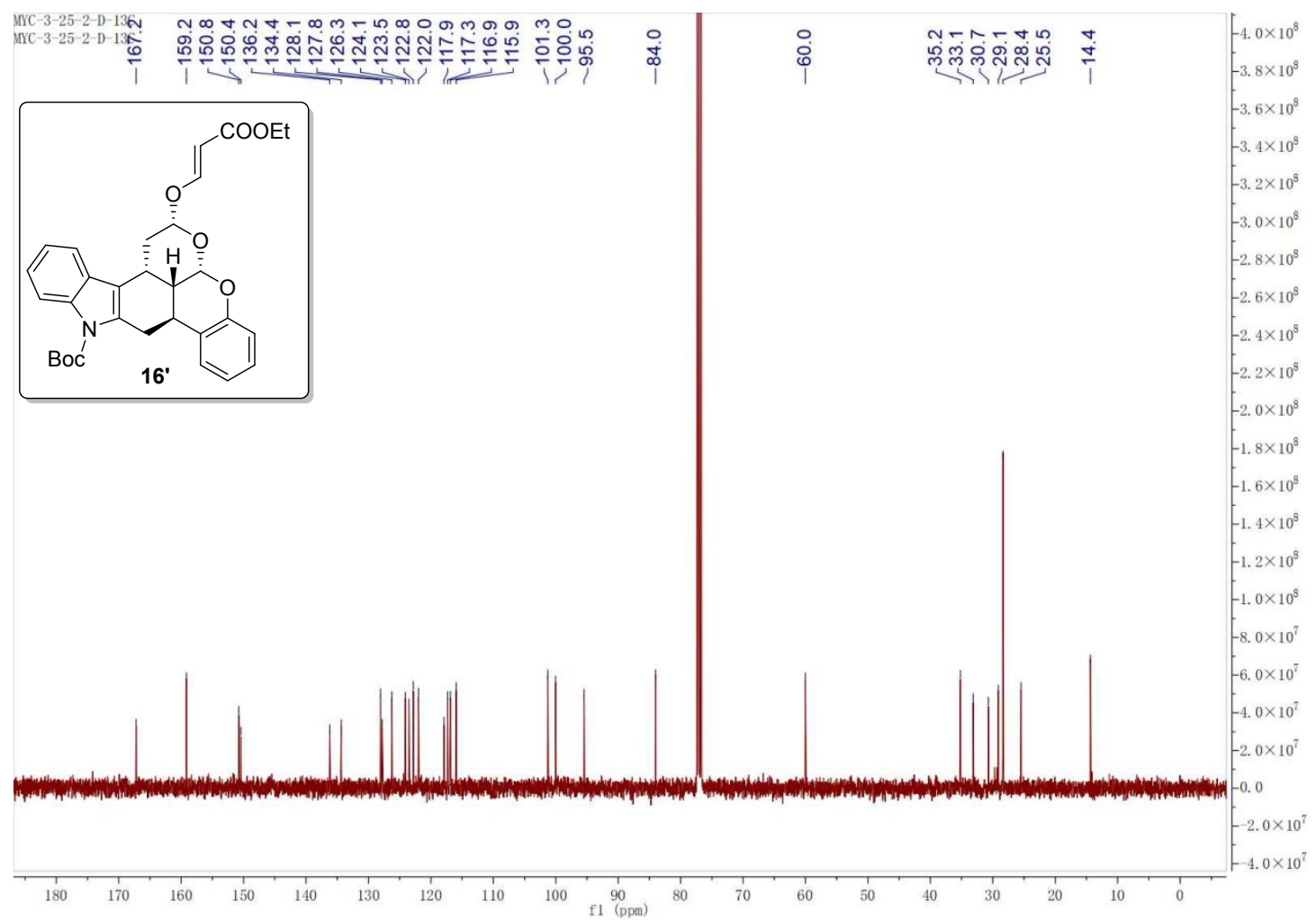

The NOSEY spectrum of 16' (500 $\left.\mathrm{MHz}, \mathrm{CDCl}_{3}\right)$

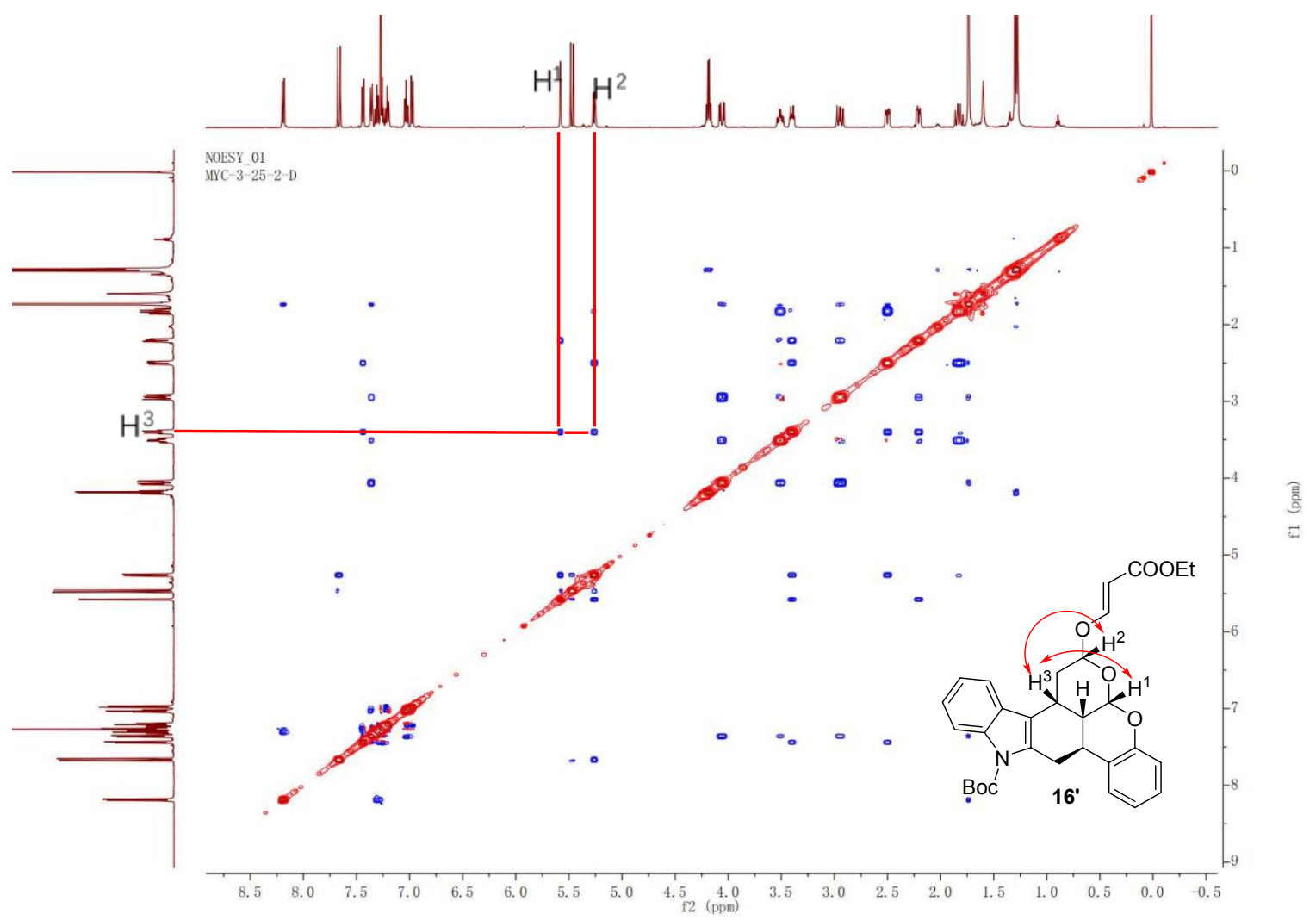




\section{The HPLC of racemic 16'}

Chrom Type: Fixed WL Chromatogram, $225 \mathrm{~nm}$

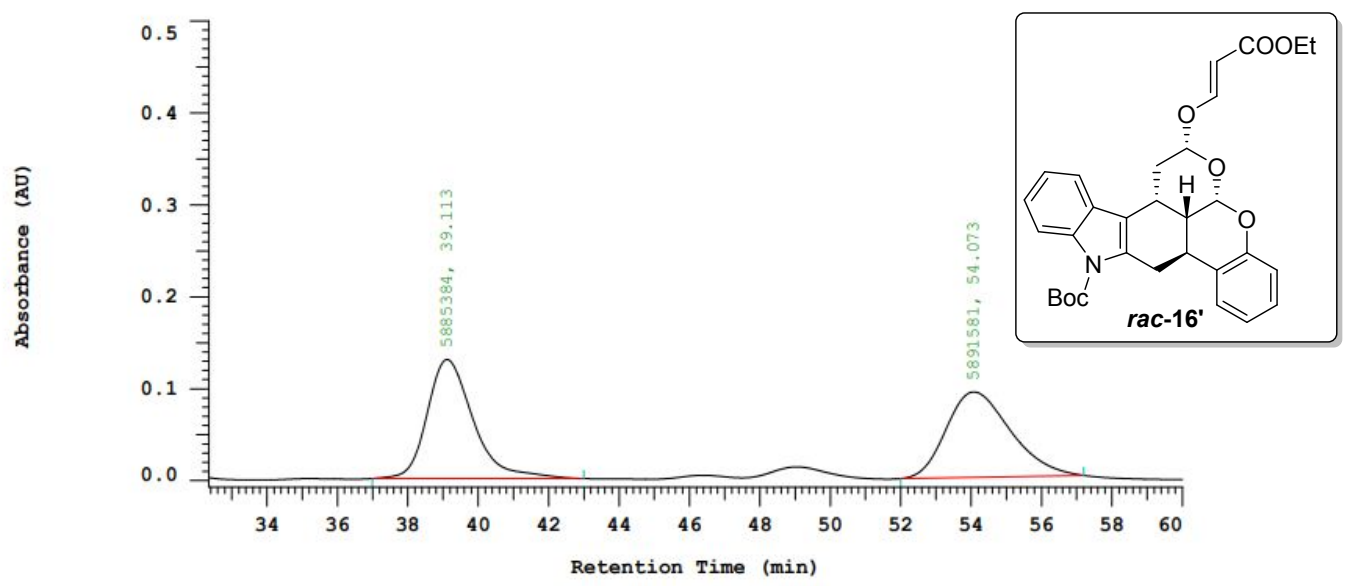

Chrom Type: Fixed WL Chromatogram, $225 \mathrm{~nm}$

Peak Quantitation: AREA

Calculation Method: AREA음

\begin{tabular}{rrrrr} 
No. & RT & Area & Area & BC \\
\hline 1 & 39.113 & 5885384 & 49.974 & BB \\
2 & 54.073 & 5891581 & 50.026 & BB \\
\hline & & 11776965 & 100.000 & \\
\hline
\end{tabular}

The HPLC of chiral 16'

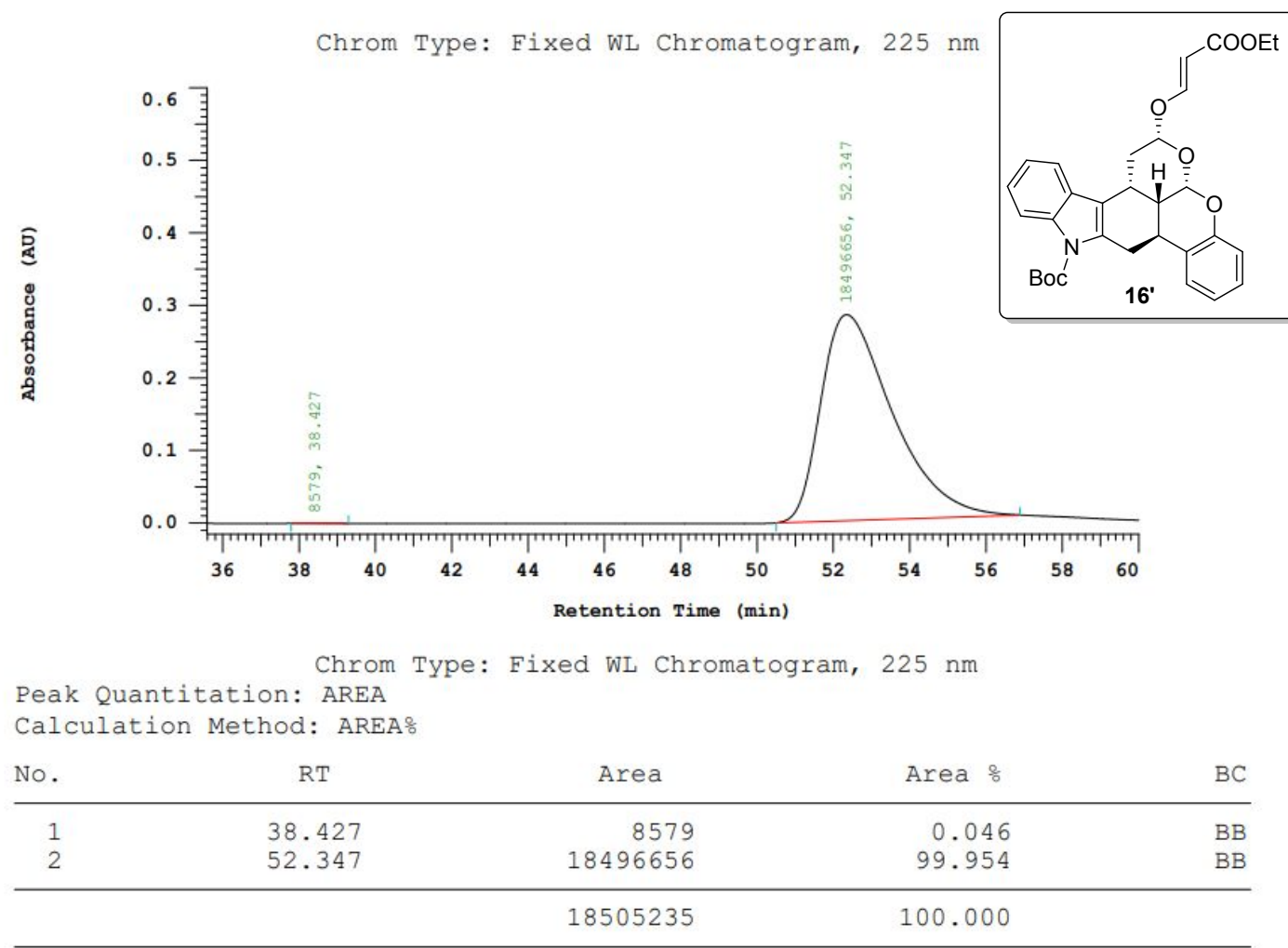




\section{K. Single crystal X-Ray diffraction data}

Single crystal preparation: 6a/9 was dissolved in $\mathrm{CH}_{2} \mathrm{Cl}_{2}(0.1 \mathrm{~mL})$, diluted with ether (1 $\mathrm{mL})$, sealed, cultured in standing, and the solvent was volatilized at room temperature. After a week, single crystals were formed.

The instrumentation used: Gemini E/Eos of Rigaku.

[CCDC 2092454 and 2092455 contain the supplementary crystallographic data for this paper. These data can be obtained free of charge from The Cambridge Crystallographic Data Centre via www.ccdc.cam.ac.uk/data_request/cif.].

Absolute configuration of 6a - CCDC 2092454

Compound 6a: Displacement ellipsoids are drawn at the 30\% probability level.
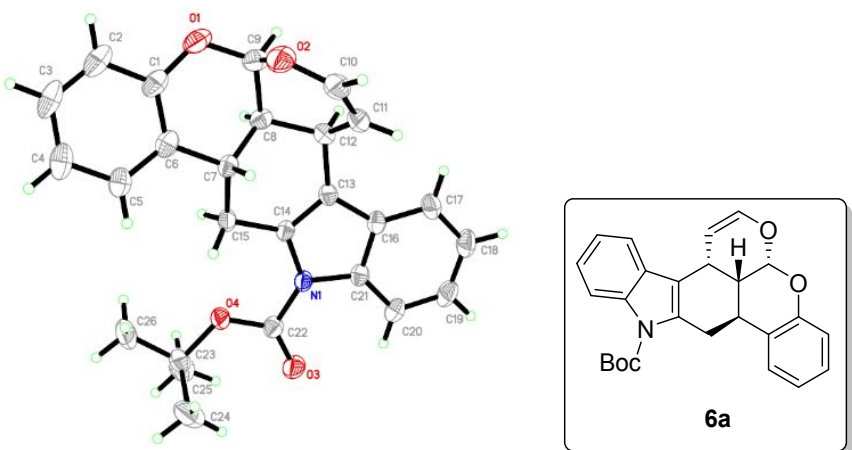


\begin{tabular}{|c|c|c|c|}
\hline Bond precision: & $C-C=0.0049 \mathrm{~A}$ & Wavelength $=$ & $=1.54178$ \\
\hline Cell: & $\begin{array}{l}a=6.6838(2) \\
\text { alpha }=90\end{array}$ & $\begin{array}{l}\mathrm{b}=10.6757(3) \\
\text { beta }=99.225(3)\end{array}$ & $\begin{array}{l}\mathrm{C}=15 \cdot 3132(5) \\
\text { gamma }=90\end{array}$ \\
\hline \multirow[t]{2}{*}{ Temperature: } & $293 \mathrm{~K}$ & & \\
\hline & Calculated & Reported & \\
\hline Volume & $1078.53(6)$ & $1078.53(6)$ & \\
\hline Space group & P 21 & P 21 & \\
\hline Hall group & P $2 y b$ & P $2 \mathrm{yb}$ & \\
\hline Moiety formula & $\mathrm{C} 26 \mathrm{H} 25 \mathrm{~N} \quad \mathrm{O} 4$ & $?$ & \\
\hline Sum formula & $\mathrm{C} 26 \mathrm{H} 25 \mathrm{~N} \quad 04$ & $\mathrm{C} 26 \mathrm{H} 25 \mathrm{~N}$ & 04 \\
\hline $\mathrm{Mr}$ & 415.47 & 415.47 & \\
\hline $\mathrm{Dx}, \mathrm{g} \mathrm{cm}-3$ & 1.279 & 1.279 & \\
\hline $\mathrm{z}$ & 2 & 2 & \\
\hline $\mathrm{Mu} \quad(\mathrm{mm}-1)$ & 0.694 & 0.694 & \\
\hline F000 & 440.0 & 440.0 & \\
\hline F000' & 441.34 & & \\
\hline $\mathrm{h}, \mathrm{k}, \mathrm{lmax}$ & $7,12,18$ & $7,12,18$ & \\
\hline Nref & $3864[2044]$ & 2762 & \\
\hline$T \min , T \max$ & $0.914,0.927$ & $0.876,1.00$ & \\
\hline Tmin' & 0.914 & & \\
\hline \multicolumn{4}{|c|}{$\begin{array}{l}\text { Correction method= \# } \\
\text { AbsCorr = MULTI-SCAN }\end{array}$} \\
\hline Data completenes & $s s=1.35 / 0.71$ & Theta $(\max )=67.221$ & \\
\hline$R$ (reflections) $=$ & $0.0383(2513)$ & wR2 (reflections) = & $0.1018(2762)$ \\
\hline$S=1.076$ & Npar $=$ & $=284$ & \\
\hline
\end{tabular}




\section{Absolute configuration of 9 - CCDC 2092455}

Compound 9: Displacement ellipsoids are drawn at the 30\% probability level.
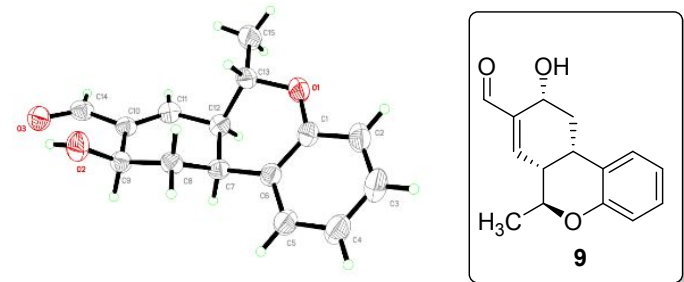

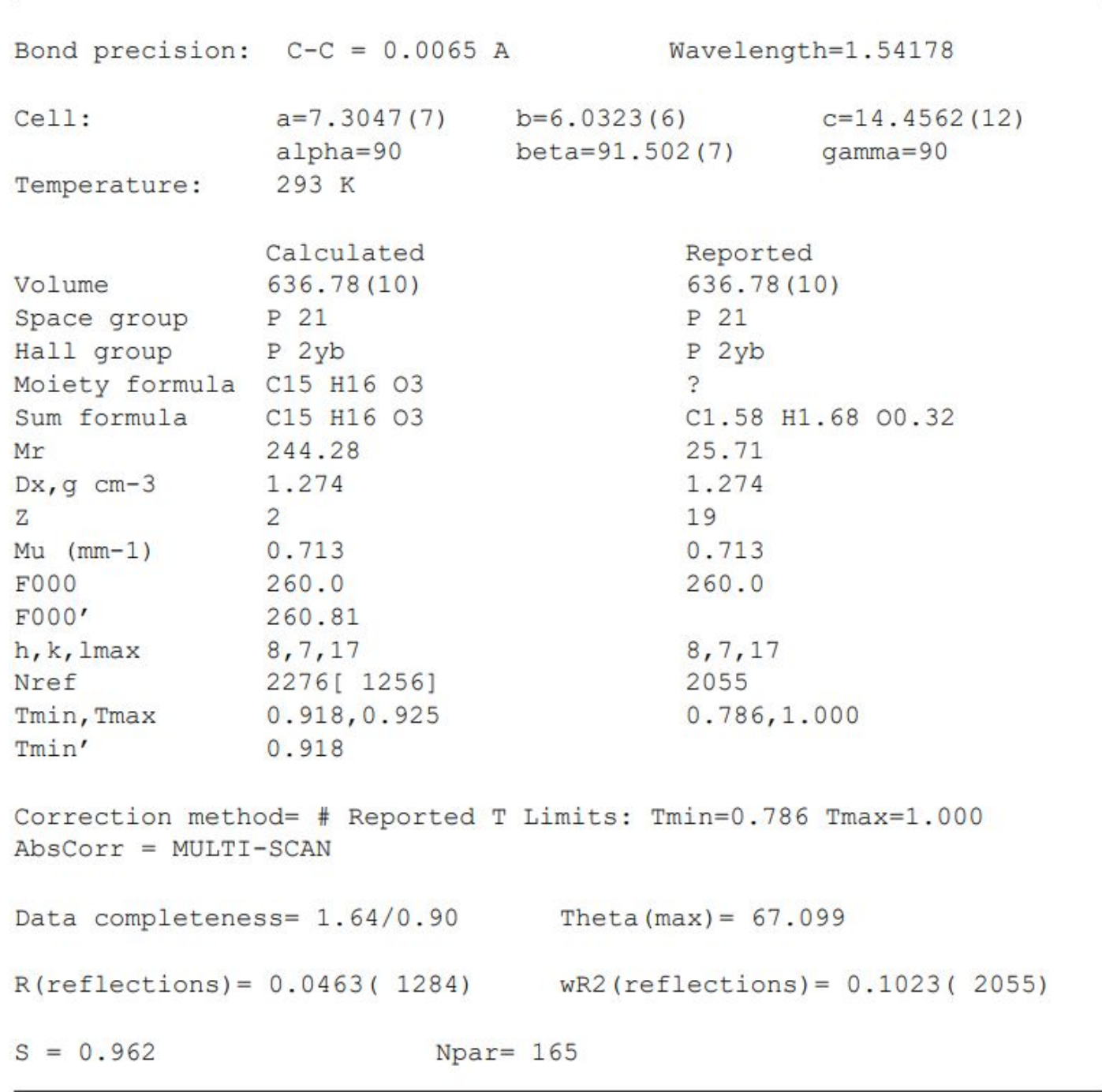




\section{Proposed reaction mechanism}

\section{Reaction sequence of $\beta$-indolyl $\alpha, \beta$-unsaturated aldehyde}

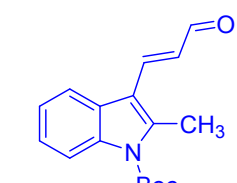

1a Boc

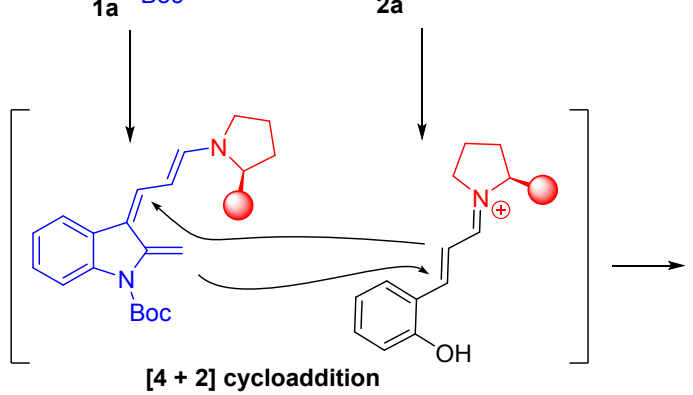

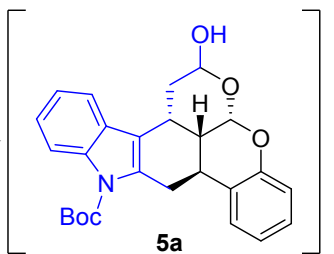

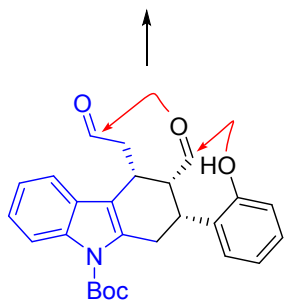

The combination of $\beta$-indolyl $\alpha, \beta$-unsaturated aldehyde 1a and aminocatalyst 3a gave a trienamine intermediate, while the combination of 2-hydroxycinnamaldehyde 2a and aminocatalyst 3a gave an iminium ion intermediate. A [4+2] cycloaddition occurred between these two active species, followed by double intramolecular hemiacetalization process to give desired product $\mathbf{5 a}$. 


\section{Reaction sequence of unbranched $\alpha, \beta$-unsaturated aldehyde $7 b$}
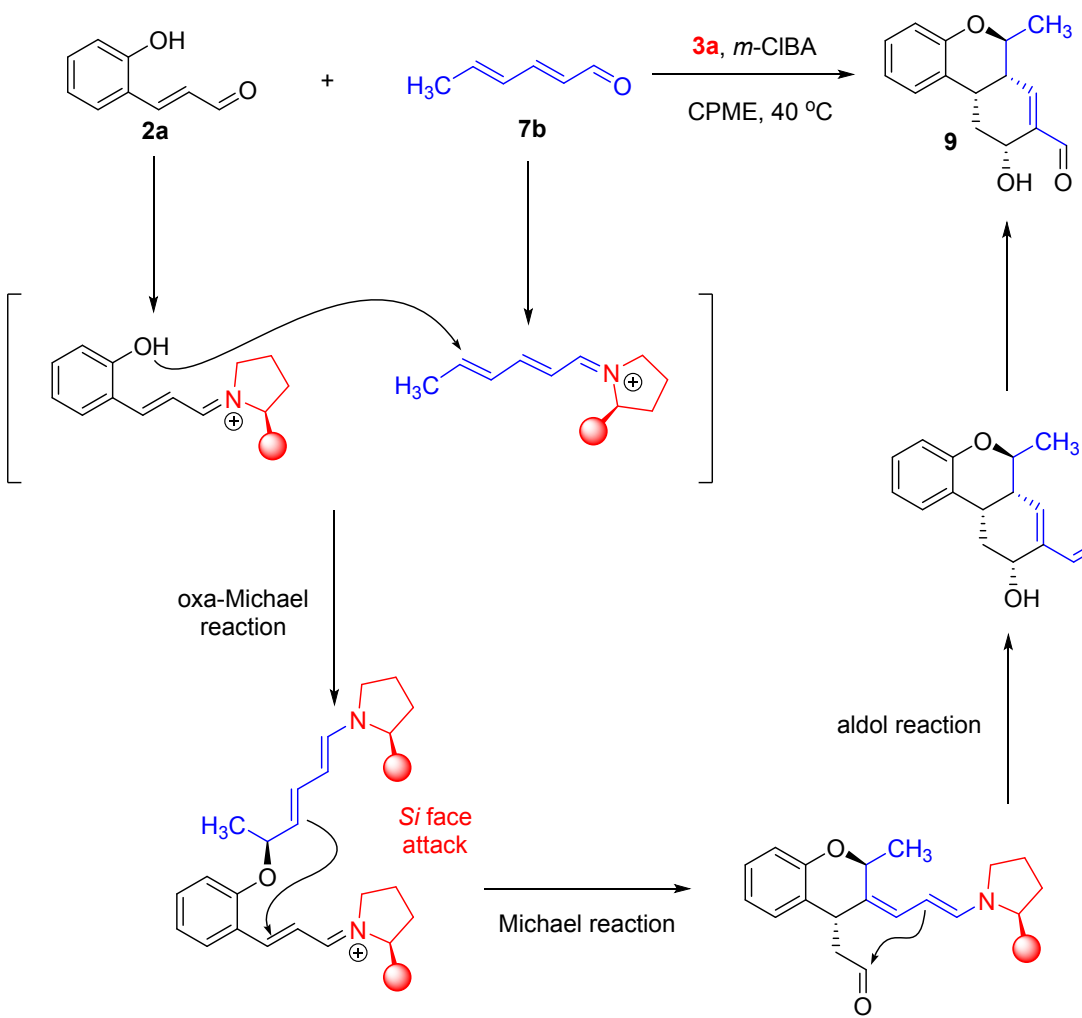

A three-step sequence was proposed: 1) oxy-Michael reaction between $\mathbf{7 b}$ and $\mathbf{2 a}$ promoted by vinylogous iminium ion activation and iminium ion activation respectively; 2) intramolecular Michael reaction promoted by dienamine activation and iminium ion activation; 3 ) intramolecular aldol reaction promoted by dienamine activation. 